\title{
Frankfurt am Main
}

Botanischer Garten der Universität und Haupt-Schulgarten der Stadt.

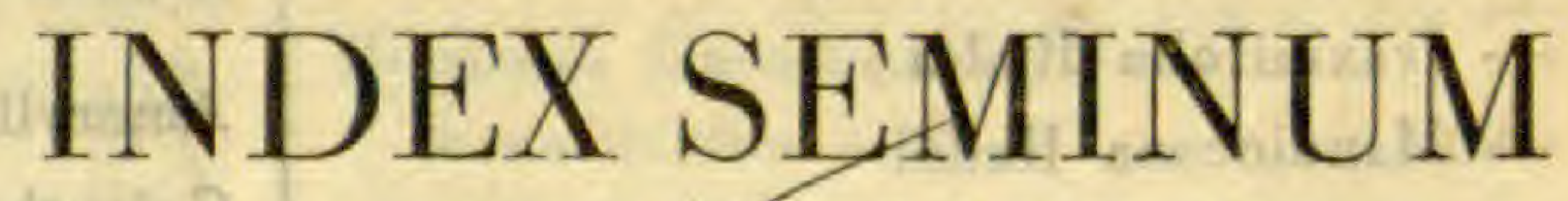

a $\mathrm{n} \quad \mathrm{n} \quad \mathrm{o} \quad 1934 \%$ o $1 \mathrm{l}$ e c t o r u m

Desiderata inscribenda sunt: An die Direktion des Botanischen Gartens, Frankfurt-M.

\section{Pteridophyta}

Cyatheaceae

Dicksonia antarctica Lab.

Marattiaceae

Angiopteris evecta Hoffm.

Osmundaceae

Osmunda regalis $\mathrm{L}$.

\section{Parkeriaceae}

Ceratopteris thalictroides Brongn. (33)

\section{Polypodiaceae}

Adiantum Capillus Veneris L.

- caudatum L.

- hispidulum Sw.

- reniforme $\mathbf{L}$.

- trapeziforme L.

Aspidium Filix mas Sw.

- melanocaulon BI.

- spinulosum Sw.

- - var. dilatatum

- trifoliatum Sw.

Asplenium Belangeri Kze.

- Nidus L.

Athyrium Filix femina Roth

Blechnum brasiliense Desv.

- Moorei C. Chr.

- Spicant Sm.

Coniogramme japonica Diels
Davallia bullata Wall.

- Tyermannii Bak.

Deparia Moorei Hook.

Diplazium Petersenii Christ

- proliferum Thouars.

- Shepherdii Link

Doodia aspera $R$. Br.

Dryopteris parasitica Kze.

- Poiteana Urb.

- Sieboldii Kze.

Fadyenia prolifera Hook.

Gymnogramme tatarica Desv.

Hemionitis arifolia Moore

Microlepia speluncae Moore

- strigosa Pr.

Niphobolus adnascens Kaulf.

Pellaea viridis PrantI

Platycerium alcicorne Desv.

- Veitchii hort.

Polypodium irioides Lam.

- Meyenianum Sehott

- vulgare $\mathrm{L}$.

Polystichum pungens Presl.

Pteris longifolia L.

- semipinnata L.

- umbrosa R. Br.

Scolopendrium Delaxayi- Faanch.

- vulgare Sm. OURI BOT

\section{JAN 301935}


Siruthiopteris germanica Willd.

Schizaeaceae

Aneimia densa Link

- Dregeana Kze.

- fraxinifolia Raddi

- Mandiocana Raddi

- Phyllitidis Sw.

- rotundifolia Schrad.

Lygodium japonicum Sw.

\section{Selaginellaceae}

Selaginella Emmeliana van Geert

- grandis Moore

- inaequalifolia Spring.

- Kraussiana A. Br.

- stenophylla A. Br.

\section{Gymnospermae}

\section{Ginkgoaceae}

Ginkgo biloba L.

\section{Pinaceae}

Biota orientalis Endl.

Chamaecyparis Lawsoniana Parl.

- pisifera Endl.

"Juniperus communis L.

Larix decidua Mill.

- leptolepis Gord.

- sibirica Ledeb.

Picea canadensis B.S.P.

Pinus excelsa Wall.

- nigra Arn.

- Strobus L.

Pseudotsuga taxifolia Britt.

Tsuga canadensis Carr.

\section{Taxaceae}

Taxus baceata L.

- - var. adpressa
Angiospermae

Monocotyledoneae

Alismataceae

Alisma Plantago $\mathbf{L}$.

Amaryllidaceae

Galanthus byzantinus Bak.

Leucojum vernum $L$.

Narcissus minor L.

- - var. nanus

Zephyranthes Atamasco Herb.

Araceae

Arum maculatum L.

Calla palustris L.

\section{Bromeliaceae}

Acanthostachys strobilacea Link Dyckia remotiflora Ott. et Dietr.

- sulphurea C. Koch

Guzmannia tricolor Ruiz. et Pav.

Lindmannia penduliflora Stapf

Tillandsia Saundersii C. Koch

Commelinaceae

Commelina clandestina Mart.

- coelestis Willd.

- communis L.

- dianthifolia Red.

Coleotryphe natalensis C. B. CI.

Palisota Barteri Hook. f.

Rhoeo discolor Hance

Tinantia Fugax Scheidw. var. erecta Drum.

Tradescantia virginiana $\mathrm{L}$.

\section{Cyperaceae}

Carex divulsa Good.

- Grayii Car.

- muricata L. 
Cyperus adenophorus Schrad.

- alternifolius L.

- esculentus L. (tub.)

- Papyrus L.

${ }^{*}$ Eriophorum polystachyum L.

\section{Gramineae}

Aegilops erassa Boiss.

Agrostis nebulosa Boiss. et Reut.

Aira capillaris Host.

Aristida capillacea Lam.

Arrhenatherum elatius M. et K.

Asperella Hystrix Humb.

Avena brevis Roth

Boissiera bromoides Hochst.

Brachypodium distachyum Beauv.

- pinnatum Beauv.

- silvaticum R, et Sch.

Briza maxima L.

Bromus asper Murr.

- commutaius Schrad.

- mollis L.

- patulus M. et K.

- pendulinus Schrad.

- sterilis L.

- tectorum L.

Catabrosa humilis Trin.

Cenchrus echinatus L.

- tribuloides $\mathrm{L}$.

Chaeturus fasciculatus Link

Coix Lacryma Jobis L.

Dactylis glomerata L.

Deschampsia caespitosa Beauy.

- flexuosa Trin.

Eragrostis pilosa Beauv.

Fesiuca arundinacea L.

- gigantea Vill.

- glanea Schrad.

- pratensis Huds.
Festuca rubra L.

- silvatica Vill.

Gaudinia fragilis Beauv.

Heleochloa alopecuroides Host.

Hordeum Gussoneanum Parl.

- hexastichum L.

- nudum L.

- Zeocrithum L.

Lolium italicum A. Br.

- remotum Schrank

- temulentum L.

Melica altissima $\mathrm{L}$.

- ciliata L.

Milium effusum L.

Molinia coerulea Moench

Panicum capillare L.

- Crus galli L.

- palmifolium Willd.

- sanguinale L.

Paspalum racemosum Lam.

Pennisetum compressum R. Br.

- typhoideum Rich.

Phalaris minor Retz

- paradoxa L.

Phleum Boehmeri Wib.

- Michelii All.

Poa compressa L.

- nemoralis L.

Polypogon monspeliensis Desf.

Setaria germanica Beauv.

- glauea Beauv.

- italica Beauv.

— viridis Beauv.

Sorghum vulgare Pers.

— — - var. nigrum

- - - saccharatum

Stipa capillata L.

- sibirica Lam.

Tragus racemosus Desf. 
Triticum dicoceum Schrank

- - var. cladurum

- monococcum L.

Zea Mays L.

- - var. oriziformis

\section{Iridaceae}

Grocus Fleischeri Gay

- pulchellus Herb.

- Sieberi Gay

- speciosus M. Bieb.

- zonatus Gay

Iris bucharica Foster

- Forrestii Dykes

- halophila Pall.

- Kaempferi Sieb.

- notha M. Bieb.

- pallida Lam.

- sibirica L.

- versicolor $\mathrm{L}$.

- warleyensis Foster

Sisyrinchium angustifolium Mill.

— iridifolium Kunth

- striatum Sm.

\section{Juncaceae}

Juncus bufonius L.

- tenuis Willd.

Luzula albida DC.

- nemorosa E. Mey.

\section{Liliaceae}

Agapanthus umbellatus L'Hérit.

Allium angulosum $\mathbf{L}$.

- azureum Ledeb.

- fistulosum L.

- flavum L.

- karataviense Rgl.

- Moly L.

- montanum Sehm.

- - var. glaueum

- oleraceum L.

- Ostrowskianum Rgl.
Allium Rosenbachianum Rgl.

- rotundum $L$.

- sativum L. (bulb.)

- Schoenoprasum L.

- ursinum $\mathbf{L}$.

- viviparum Kar. et Kir. (bulb.)

Anthericum Liliago L.

Asphodeline lutea Rehb.

Asphodelus albus Mill.

- Villarsii Verl.

Bulbine annua Willd.

Bulbocodium vernum $L$.

Camassia esculenta Lindl.

Chionodoxa Luciliae Boiss.

Chlorophytum amaniense Engl.

- ukambense Bak.

Colchicum autumnale L.

- Bornmuelleri Freyn.

- speciosum Stev.

Convallaria majalis L.

Eremurus Bungei Bak.

- himalaicus Bak.

- Kaufmannii Rgl.

- robustus $\mathrm{Rgl}$.

Fritillaria imperialis L.

- Meleagris L. (33)

Galtonia candicans Dene.

Haemanthus albiflos Jacq.

Hosta coerulea Tratt.

- plantaginea Aschs.

- Sieboldiana Engl.

Hyacinthus amethystinus L.

Lilium bulbiferum L.

- Henryi Bak.

- Martagon L.

- regale Wils.

- superbum L.

Majanthemum bifolium Sehmidt 
Muscari botryoides Mill.

- comosum Mill. (33)

Nothoscordum fragrans Kunth

Polygonatum multiflorum All.

- officinale All.

Scilla bifolia L.

- sibirica Andr.

Tofielda palustris Huds. (33)

Tricyrtis macropoda Miq.

Tulipa Kaufmanniana Rgl.

- Marjolettii Per. et Song.

- silvestris L.

- Sprengeri Bak.

Urginea maritima Bak.

Veratrum album L.

- nigrum $\mathbf{L}$.

\section{Scheuchzeriaceae}

Triglochin maritima L.

* - - (Hiddensee)

- palustris $\mathrm{L}$.

Taceaceae

Tacea macrantha Jacq.

Zingiberaceae

Alpinia calcarata Rose.

\section{Dicotyledoneae}

Acanthaceae

Acanthus longifolius Host

Hemigraphis primulaefolia Vill.

Ruellia squarrosa Fenzl.

- strepens L.

Strobilanthes roseus Nees.

Thunbergia alata Boj. (33)

\section{Aceraceae}

Acer cappadocicum Gled.

- circinatum Pursh.
- Ginnala Maxim.

- macrophyllum Pursh.

- monspessulanum L.

- tataricum L.

Aizoaceae

Mesembrianthemum eriniflorum Houth.

- linguiforme $\mathrm{L}$.

- pinnatifidum $\mathrm{L}$.

- spectabile Haw.

- vespertinum Berg.

Tetragonia echinata Ait.

- expansa Ait.

\section{Amarantaceae}

Achyranthes aspera L.

Alternanthera sessilis R. Br.

Amarantus albus L.

- Blitum L. (33)

- caudatus L.

- flavus L.

- retroflexus L.

- spinosus L.

Gomphrena decumbens Iacq.

- globosa L.

Telanthera polygonoides Moq.

Anacardiaceae

Rhus typhina L.

- vernicifera DC.

Apocynaceae

Amsonia Tabernaemontana Walt.

Aquifoliaceae

Ilex Aquifolium L.

- glabra Gray

Araliaceae

Aralia racemosa L.

- spinosa L.

Aristolochiaceae

Aristolochia Clematitis L. 
Asarum europaeum L.

- - var. caucasicum Duch.

Asclepiadaceae

Asclepias Cornuti Dene.

- curassavica L.

- inearnata $\mathrm{L}$.

Ceropegia debilis N. E. Br. (tub.)

-Woodii Schlecht. (tub.)

Dischidia pectenoides Pears.

Stapelia mutabilis Jacq.

— variegata $L$.

Vincetoxicum officinale Moench

Balsaminaceae

Impatiens Balfourii Hook. f.

- Balsamina L.

- biflora Walt.

- Holstii Engl. et Warb.

- Roylei Walp.

Basellaceae

Basella alba L.

- rubra L.

Boussingaultia baselloides $H$. B. et K.

\section{Berberidaceae}

Berberis Thunbergii DC.

- Veitchii C. Schneid.

- vulgaris $\mathrm{L}$.

- Wilsonae Hemsl.

Podophyllum Emodi Wall.

\section{Betulaceae}

Alnus cordata Desf.

- glutinosa Gaertn.

- incana Moench

Betula lenta L.

- pubescens Ehrh.

\section{Bignoniaceae}

Catalpa bignonioides $W$ alt.

- ovata G. Don

(rhiz.)
Incarvillea compacta Max. var. brevipes

- Delavayi Bur. et Franch.

- Olgae Rgl.

\section{Borraginaceae}

Alkanna lutea A. DC.

Anehusa officinalis L.

Borrago laxiflora DC.

* Cerinthe alpina Kit. (Allgäu)

- major L.

- minor $\mathrm{L}$.

Cynoglossum amabile Stapf et Drum.

- coelestinum Lindl.

- officinale L.

- pictum Ait. (33)

Echinospermum Lappula Lehm.

Echium plantagineum L.

- vulgare L.

Heliotropium europaeum L.

Lindelofia anchusaefolia Lehm.

- longifolia Guerke

Lithospermum officinale $\mathrm{L}$.

Lycopsis arvensis $\mathrm{L}$.

Omphalodes cappadocica DC.

- linifolia Moench

Onosma stellulatum W. et K.

Solenanthus apenninus Hohen.

Calycanthaceae

Galycanthus floridus L.

- praecox L. (33)

\section{Campanulaceae}

Adenophora latifolia Fisch.

- liliifolia Ledeb.

Campanula alliariifolia Willd.

- Aucheri DC.

- barbata L. (33)

- carpatica Jacq.

- Cervicaria L. (33)

- drabifolia Sibth. et Sm. 
Campanula garganica Ten.

— - var. hirsuta hort.

- glomerata L. var. dahurica Fisch.

- lactiflora Bieb.

- latifolia L.

- patula L.

- persicifolia L.

- - var. macrautha DC.

- Portenschlagiana R. et Sch.

- pusilla Haenke

- pyramidalis $\mathbf{L}$.

- Raddeana Trautv.

- ramosissima Sibth. et Sm.

- rapunculoides L.

- rotundifolia $\mathrm{L}$.

- sarmatica Ker. Gawl.

- sibirica L. (33)

- Trachelium L.

- turbinata Schott.

- velutina Desf.

Codonopsis clematidea C. B. CL.

- viridiflora Maxim.

Hedraeanthus dalmaticus A. DC.

Jasione montana L.

- perennis Lam.

Isotoma axillaris Lindl.

Lobelia inflata L.

- ramosa Benth.

- syphilitica L.

Phyteuma canescens W. et K.

- nigrum Schmidt

- orbiculare L.

- Scheuchzeri All.

- spicatum L.

Platycodon grandiflorum A. DC.

Podanthum limonifolium Boiss.

Specularia falcata A. DC.

- hybrida A. DC.

- pentagonia A. DC.

- Speculum A.DC.

Symphyandra Hofmannii Pant.
Wahlenbergia gracilis A. DC.

- pendula Schrad.

Capparidaceae

Cleome pungens Willd.

Polanisia graveolens Rafin.

\section{Caprifoliaceae}

Sambucus Ebulus L.

Viburnum Carlesi Hemsl.

- Lantana L.

- Opulus L.

Caryophyllaceae

Agrostemma Githago L.

Alsine laricifolia Wahl.

- tenuifolia Wahl.

Anychia dichotoma Mehx. (33)

Arenaria graminifolia Schrad.

Cerastium chlorifolium F. et M.

- tomentosum L.

Cucubalus baccifer L.

Dianthus Armeria L.

- banaticus Heuff.

- Carthusianorum L.

- cruentus Griseb.

- deltoides $\mathrm{L}$.

- pelviformis Heuff.

- plumarius L.

- Seguierii Vill.

- trifasciculatus Kit.

Gypsophila altissima L.

- paniculata L.

- repens L.

Heliosperma alpestre Rehb.

Lychnis alpina $\mathbf{L}$.

- chalcedonica L.

- Coronaria Desr.

- Flos euculi L.

- Flos Jovis Desr.

- fulgens Fisch.

- Viscaria L. 
Melandryum album Garcke (33)

- rubrum Garcke

Moehringia trinervia Clairv.

Polycarpon tetraphyllum L.

Saponaria cerastioides Fisch. et Mey.

- ocymoides L.

- officinalis L.

- orientalis L.

Seleranthus annuus $\mathrm{L}$.

- perennis L.

"Silene acaulis L. (33)

- Armeria L.

- echinata Otth.

- Friwaldskyana Hampe

- gallica L.

- italica Pers.

- longiflora Ehrh.

- multicaulis Guss.

- nutans L.

- quinquevulnera L.

- Schafta Gmel.

- vulgaris Garcke

Spergula arvensis L.

*Tunica prolifera Scop.

- Saxifraga Scop.

- velutina Fisch. et Mey.

Vaccaria pyramidata Med.

\section{Celastraceae}

Evonymus alata Rgl.

- europaea, L.

- latifolia Scop.

- nana M. Bieb.

- radicans Miq.

\section{Chenopodiaceae}

Atriplex nitens Schkuhr.

Chenopodium ambrosioides $\mathrm{L}$.

- Bonus Henricus L.

- Botrys L.

- foliosum Aschs.

- polyspermum L.
Chenopodium Quinoa L.

- rubrum L.

- Vulvaria L.

Corispermum hyssopifolium L.

Hablitzia tamnoides M. Bieb.

Kochia arenaria Roth

Monolepis trifida Schrad.

Salsola Kali L.

- Soda L.

\section{Cistaceae}

Helianthemum hirtum Mill.

Clethraceae

Clethra acuminata Michx.

- alnifolia L.

\section{Compositae}

Achillea Millefolium L.

- nana $L$.

- ptarmica L. (33)

Ambrosia maritima L.

Ammobium alatum R. Br.

Anacyclus officinarum Hayne

Anaphallis yedoensis Maxim.

Antennaria neodioica Greene

Anthemis tinctoria L.

Aretotis grandis Thunb.

Arnica montana L.

Artemisia Absinthium L. (33)

- annua L.

- sacrorum Ledeb. var. viridis

- vulgaris L. (33)

Aster alpinus L.

- ptarmicoides Torr. et Gray

- umbellatus Mill.

Baeria gracilis Gray

Bellium bellidioides $\mathbf{L}$.

Bidens leucanthus Willd.

- tripartitus L. 
Brachycome iberidifolia Benth. (33)

- pachyptera Turcz.

Buphthalmum salieifolium L.

- speciosum Schreb.

Calendula arvensis $\mathrm{L}$.

- officinalis L.

Callistephus chinensis Nees „Helvetia“

Carduus acanthoides L.

* nutans L.

Carlina acaulis $\mathrm{L}$.

Carthamus tinctorius $\mathbf{L}$.

Centaurea Crocodylium L

- dealbata Willd.

- eriophora L.

- involucrata Desf.

- Lippii L.

- macrocephala Puschk.

- montana L.

- nigra L. (33).

* rhenana Bor. (Flörsheim)

- Scabiosa L.

* solstitialis L.

- sulphurea Willd.

Cephalophora aromatica Schrad.

Charieis heterophylla Cass.

Chrysanthemum cinerariifolium Trev.

- corymbosum L.

- Leucanthemum L.

- macrophyllum W. et K.

- vulgare Bernh.

Chrysocoma Coma aurea L. (33)

Chrysopsis villosa Nutt.

Cichorium Intybus L.

Cirsium eriophorum Scop.

- Velenowskyi Vand.

Cnicus benedictus L.

- syriacus Roth

Coreopsis Drummondii Torr. et Gray

- lanceolata L. (33)

- tripteris $\mathrm{L}$.
Coreopsis verticillata $\mathrm{L}$.

Cosmos atrisanguineus Hook. (33)

Cotula coronopifolia L. (33)

- turbinata L. (33)

Crepis aurea Cass.

- blattarioides Vill.

- montana Tausch.

- sibirica L.

Crupina pauciflora Hoffm. et Link

Crysostemma calendulaceum R. Br. (33)

Cynara Scolymus L.

Doronicum Pardalianches L.

Echinacea purpurea Hook.

Echinops sphaerocephalus L.

$\%$

Eclipta erecta L.

Emilia flammea Cass.

Eriophyllum caespitosum Dougl.

Eupatorium purpureum L.

Felicia fragilis Cass. (33)

Filago germanica L.

Gamolepis Tagetes DC.

Gazania longiscapa DC.

*Gnaphalium silvaticum L.

Guizotia abyssinica Cass.

Hedypnois cretica Willd. (33)

Helenium Hoopesi Gray

- puberulum DC. (33)

- tenuifolium Nutt.

Helianthus annuus L.

- - f. purpureus

Helichrysum foetidum Moench

- orientale Gaertn.

Heliopsis helianthoides Sweet.

Helipterum roseum Benth.

Hieracium amplexicaule L.

- aurantiacum L. 
Hieracium Bornmuelleri Frayn.

- bupleuroides Gmel.

- humile Jacq.

- laevigatum Willd.

- murorum L.

- Pilosella L.

- silvestre Tausch.

Hypochoeris glabra L.

- radieata $\mathrm{L}$.

- uniflora Vill.

\%—_- (Allgäu)

Inula Conyza DC.

- germanica L.

- glandulosa Pusehk.

- Helenium L.

Jaegeria hirta Less.

Koelpinia linearis Pall.

Lactuca altissima Bieb. (33)

- muralis Less. (33)

- Scariola L.

- virosa $\mathrm{L}$.

Lappa major Gaertn.

- minor DC.

- tomentosa All. (33)

Leptosyne maritima A. Gray. (33)

Liatris spicata Willd.

Ligularia macrophylla DC.

Matricaria Chamomilla L.

Mieroseris Lindleyi A. Gray

- pygmaea D. Don

Mulgedium Burgaei Boiss.

- Plumieri DC.

- sibiricum Less.

Myriactis nepalensis Less.

Onopordon Acanthium L.

*

Palafoxia texana DC.

Petasites albus Gaertn.

Picridium tingitanum Desf.
Picris echioides L.

*

Pulicaria dysenterica Gaertn.

Pyrethrum carneum Bieb.

Rhagadiolus stellatus Willd.

Rhaponticum pulchrum Fisch. et Mey.

Rudbeckia amplexicaulis Vahl.

- bicolor Nutt.

- hirta L.

- laciniata L. (33)

- purpurea L.

- speciosa Wend.

Sanvitalia procumbens Lam.

Senecio clivorum Maxim.

- Fuchsii Gmel.

- Veitchianus Hemsl.

Siegesbeckia orientalis L.

Silphium perfoliatum L.

Silybum Marianum Gaertn.

Sonchus paluster L.

Solidago Virg-aurea L.

th

Stevia serrata Cav. (33)

Thelesperma Burridgeanum hort. (33)

Tithonia tagetiflora Desf.

Tolpis barbata Gaertn.

Tragopogon major Jacq.

- porrifolius $\mathbf{L}$.

Tridax trilobata Hemsl.

Ursinia anethoides N. E. Br.

Verbesina encelioides Benth. et Hook.

Xanthium spinosum L.

- strumarium L.

Xeranthemum inapertum Mill.

Zazintha verrucosa Gaertn.

Zinnia paueiflora L.

- verticillata Andr. 


\section{Convolvulaceae}

Convolvulus elongatus Willd.

- pentapetaloides L.

- tricolor L.

- undulatus Gav.

Cuscuta Epilinum Weihe

- Gronowii Willd.

Ipomoea purpurea Roth

Quamoclit coccinea Moench var. hederifolia House

- pinnata Boj.

\section{Cornaceae}

Cornus mas L.

- pubescens Nutt.

- tatarica Mill. var. sibirica Lodd.

Crassulaceae

Chiastophyllum oppositifolium Berg.

Kalanchoe Welwitschii Britt.

Sedum acre L. (33)

- Aizoon L.

- album L.

- hybridum L.

- kamtschaticum Fisch. et Mey.

- maximum Sut.

- populifolium Pall.

- reflexum L.

- spurium M. Bieb.

\section{Cruciferae}

Aethionema armenum Boiss.

- pulchellum Boiss. et Huet.

Alyssum argenteum All.

- Moellendorfianum Aschs.

- petraeum Ard.

- saxatile L.

Arabis alpina $\mathrm{L}$.

- arenosa Scop. (33)

- hirsuta Scop. (33)

- perfoliata Lam.

- Turrita L. (33)
Aubrietia deltoidea DC.

Barbaraea vulgaris $\mathrm{R}$. Br.

Biscutella laevigata L.

Brassica nigra Koch

- Rapa L. var. annua Koch

Braya supina Koch

Camelina sativa Crantz

Capsella Bursa pastoris Moench

- Viguierii Blar.

Cochlearia officinalis L.

Coronopus procumbens Gilib.

- Ruellii All.

Crambe cordifolia Stev.

— - var. palmatifida D. E. S.

- grandiflora DC.

- koktebelica Busch (33)

- maritima L.

Diplotaxis erucoides DC.

- muralis DC.

\#_ tenuifolia DC. (Mainz)

Draba Aizoon Wahl.

Eruca sativa Lam.

Erysimum cheiranthoides L.

- odoratum Ehrh.

- Perowskianum F. et M.

Hesperis matronalis L.

Iberis amara $\mathrm{L}$.

- Lagascana DC.

- sempervirens L.

- Tenoreana DC.

Isatis tinctoria L.

Lepidium latifolium L.

- sativum L.

Lunaria biennis Moench (33)

- rediviva $\mathrm{L}$.

Malcolmia africana Ait.

- chia DC.

- maritima Ait. (33)

Matthiola bicornis DC. 
Neslea paniculata Desv.

Peltaria alliacea Jacq.

Raphanus sativus L, var. oleifer

Rapistrum rugosum All.

Schivereckia podoliea Andr.

Sinapis alba L.

Sisymbrium officinale Scop.

- strictissimum L.

Stenophragma Thalianum Cel.

Vesicaria utrieulata Lam.

\section{Cucurbitaceae}

Bryonia dioica Jaeq.

Cucumis metuliferus E. Mey.

- myriocarpus Naud.

Cucurbita ficifolia Bché.

- maxima Duch. var. turbaniformis

Cyclanthera explodens Naud.

- pedata Schrad.

Eeballium Elaterium A. Reh.

Echinocystis Wrightii Cogn.

Melothria pendula L.

Datiseaceae

Datisea cannabina L.

\section{Dipsacaceae}

Cephalaria alpina Schrad.

- tatarica Sehrad.

Dipsacus Fullonum L.

- pilosus L.

- silvester Huds.

Knautia arvensis Coult.

Morina longifolia Huds.

Scabiosa Columbaria L.

- - var. ochroleuea

\section{Droseraceae}

Drosera capensis L.
*Drosera rotundifolia L.

- spathulata Labill.

\section{Elaeagnaceae}

Hippophae rhamnoides L.

\section{Empetraceae}

"Empetrum nigrum L. (Rhön)

\section{Ericaceae}

Bruckenthalia spiculifolia Rehb.

Calluna vulgaris Salisb.

Dabeocia cantabrica K. Koch

Erica stricta Andr.

Kalmia latifolia L.

Rhododendron maximum L.

Vaccinium Vitis Idaea L.

\section{Euphorbiaceae}

Acalypha brachystachya Hornem.

- virginiana $\mathrm{L}$.

Euphorbia bubalina Boiss.

- Cyparissias L.

- exigua L.

- Gerardiana Jacq.

- heterophylla L.

- Lagascae Spreng.

- Lathyris L.

- Myrsinitis L.

- platyphyllos L.

- stricta L.

Jatropha podagraria Hook.

Manihot utilissima Pohl

Mercurialis annua L.

Ricinus communis L.

Securinega flueggeoides Miill.-Arg.

\section{Gentianaceae}

Erythraea Centaurium Pers.

Gentiana asclepiadea L.

- cruciata L. 
Gentiana dahurica Fisch.

* punetata L. (Allgäu)

- tibetica King

Menyanthes trifoliata L.

\section{Geraniaceae}

"Erodium cicutarium L'Hérit.

- gruinum Ait.

- Manescavii Boub.

- moschatum L'Hérit.

- romanum Willd.

Geranium anemonifolium L'Hérit.

- molle L.

- pratense L.

- - f. albiflorum

- Robertianum L.

- rotundifolium L.

- silvaticum L.

Gesneraceae

Alloplectus sanguineus Mart.

Besleria grandiflora H. B. et K.

Klugia zeylanica Gardn.

Streptocarpus Rexii Lindl. hybr.

\section{Globulariaceae}

Globularia Willkommii Nym.

\section{Guttiferae}

Hypericum Ascyron L.

- empetrifolium Willd.

- hircinum L. (33)

- hirsutum L.

- patulum Thunb. (33)

- perforatum L.

\section{Hydrophyllaceae}

Nemophila maculata Benth.

Phacelia campanularia A. Gray

- congesta Hook.

- malvaefolia Cham. et Schlecht.

- tanacetifolia Benth.

- Whitlavia A. Gray

\section{Labiatae}

Ajuga Chamaepitys Sehreb.

Ballota nigra $\mathrm{L}$.

Brunella grandiflora Jacq.

- vulgaris $\mathrm{L}$.

Calamintha Clinopodium Spen.

- Nepeta Clairv.

Cedronella cana Hook.

Dracocephalum altaiense Laxm.

- Moldavica L.

- Ruyschiana L.

Dysophylla verticillata Benth.

Elsholtzia cristata Willd.

- Stauntonii Benth.

Galeopsis ochroleuca Lam.

- speciosa Mill.

- Tetrahit L.

Horminum pyrenaicum L. (33)

Hyssopus officinalis L.

Koellia virginiana MacM.

Lallemantia iberica F. et M.

Lamium album L.

- Galeobdolon Crantz

- maculatum L.

- Orvala L.

Lavendula vera $L$.

Leonurus Cardiaca L.

- sibiricus L.

Lycopus europaeus L. (33)

*Marrubium vulgare L.

Melissa officinalis $\mathbf{L}$.

Melittis Melissophyllum L.

Mentha longifolia Huds.

Micromeria rupestris Benth. (33)

Moluccella laevis L.

Monarda fístulosa $\mathrm{L}$. 
Nepeta Cataria L.

- grandiflora M. Bieb.

- japonica Maxim.

- nuda L.

- Veitchii Duth. (33)

Ocimum Basilicum L.

Origanum Majorana L.

- vulgare $\mathrm{L}$.

Phlomis tuberosa L.

Physostegia virginiana Benth.

Plectranthus Oertendahlii Th. Fries

Salvia argentea L.

- Bulleyana Diels

- cleistogama De Bary

- coccinea Juss.

- farinosa Benth.

- glutinosa L.

- Jurisicii Kos.

- nemorosa L.

- officinalis L.

- patens Cav.

- pratensis L.

- Roemeriana Scheele

- Schiedeana Stapf

- silvestris L.

- verticillata L.

Satureja alpina Briq. (33)

- hortensis L.

Scutellaria albida L. (33)

- alpina L.

- baikalensis Georgi

- columnae All.

- galericulata L.

Sideritis montana L.

Stachys Alopecurus Benth.

- Betonica Benth.

- germanica L.

- grandiflora Benth.

- lanata Jaeq. (33)

- officinalis Trev.

- silvatiea L.
Teucrium Botrys L.

- flavum L.

- hircanicum L. (33)

- Scorodonia L.

Thymus Serpyllum I.

- vulgaris L.

\section{Lardizabalaceae}

Decaisnea Fargesii Franch.

\section{Leguminosae}

Abrus precatorius L.

Amorpha fruticosa L. (33)

*Anthyllis Vulneraria L. (Rhön)

Astragalus baeticus L.

- glycyphyllos L.

- danicus Retz. (33)

Baptisia australis R. Br.

Caragana arborescens Lam.

Cercis Siliquastrum L.

Cicer Arietinum L.

Cladrastis lutea K. Koch

Colutea arborescens $\mathrm{L}$.

Coronilla scorpioides Koch

- varia L.

Cytisus capitatus Jacq.

- nigricans L.

Desmodium canadense DC.

Dolichos ornatus Wall.

Galega officinalis L.

Genista tinctoria L.

Glycine ussuriensis Rgl. et Mack.

Glycyrrhiza echinata L. (33)

Halimodendron argenteum Fisch.

Hippocrepis multisiliquosa $\mathrm{L}$.

Hosackia Purshiana Benth.

Indigofera Gerardiana Wall.

Laburnum vulgare Grisb. 
Lathyrus Aphaca L.

- Gicera L. (33)

- Clymenum L.

- hirsutus $\mathrm{L}$.

- latifolius L.

- niger Bernh.

- Ochrus DG.

- silvester L.

- tingitanus L.

- vernus Bernh.

Lotus corniculatus L.

- ornithopodioides L.

- Tetragonolobus L.

Lupinus angustifolius L.

- luteus L.

- ornatus Dougl.

- perennis L.

Medicago denticulata Willd.

- hispida Gaertn.

- Iupulina L.

- orbicularis All.

- sativa L. (33)

- scutellata All.

- tribuloides Lam.

- tuberculata Willd.

Melilotus albus Med.

- caeruleus Lam.

- officinalis Desr.

Mimosa pudica L.

Onobrychis sativa Lam.

Ononis hireina Jacq.

- spinosa L.

Ornithopus compressus L.

- sativus Brot.

Oxytropis campestris DC.

Phaseolus Iunatus L.

- multiflorus L.

- vulgaris L. var. cerasiformis

- - - lilacinus

- - - nigerrimus

Pisum arvense $\mathrm{L}$.
Psoralea Onobrychis Nutt.

Robinia Pseudacacia L.

Sarothamnus scoparius Wimm.

Scorpiurus suleata L.

Soja hispida Moench var. brunea

- - - lutea

- - - nigra

Sophora japonica L.

Thermopsis fabacea DC.

"Trifolium fragiferum L. (Hiddensee)

- hybridum L.

- incarnatum $\mathbf{L}$.

- Lupinaster L.

- ochroleucum L.

- pratense L.

- repens $\mathrm{L}$.

- rubens L.

Trigonella Foenum graecum L.

Vicia dumetorum L.

- Ervilia Willd.

- Faba L.

- - var. equina

- grandiflora Scop.

- monanthos Desf.

- sativa $L$.

\section{Limnanthaceae}

Limnanthes alba Hartw.

Linaceae

Linum alpinum L. (33)

- angustifolium Huds.

- austriacum L.

- capitatum Kit.

- flavum L. (33)

- grandiflorum Desf.

- nervosum W. et K.

- perenne L. (33)

- usitatissimum L.

Loasaceae

Blumenbachia Hierouymi Urb. 
Cajophora lateritia Presl.

Loasa ferruginea Urb. et Gilg.

- hispida $\mathbf{L}$.

- parviflora Schrad.

- tricolor Wum.

- vuleanica Andr.

\section{Lythraceae}

Cuphea lanceolata Ait.

- - var, coccinea

- viscosissima Jacq.

Epilobium angustifolium L.

- Hectori Hausskn.

- hirsutum L.

- palustre L.

- parviflorum Schreb.

Lythrum Salicaria L.

\section{Malvaceae}

Abutilon Avicennae Gaertn.

Althaea cannabina L.

- officinalis L.

- rosea Gav.

- - var. nigra

Anoda Dilleniana Cav.

Callirrhoe pedata A. Gray

Gossypium herbacum L.

Hibiscus Trionum L.

Kitaibelia vitifolia Willd.

Lavatera thuringiaca L.

- trimestris L.

Malva Alcea L.

- crispa L.

- mauritiana L.

- moschata L.

*

- neglecta Wallr.

Pavonia spinifex Cav.

\section{Martyniaceae}

Martynia Intea Lindl.

- proboscidea Glox.

\section{Melastomataceae}

Bertolonia maculata DC.

- pubescens hort.

\section{Moraceae}

Dorstenia Contrajerva L.

Humulus Lupulus L. (33)

Myricaceae

Myrica cerifera L.

\section{Myrsinaceae}

Ardisia erispa A. DC.

Myrtaceae

Psidium Cattleyanum Sab.

\section{Nolanaceae}

Nolana atriplicifolia D. Don

- prostrata L.

\section{Nyctaginaceae}

Mirabilis divaricata Lowe

- jalapa L.

- longiflora L. (33)

Qenotheraceae

Circaea cordata Royle (33)

- lutetiana L.

Clarkia elegans Dougl.

Eucharidium grandiflorum F. et M.

Godetia amoena G. Don.

- dasycarpa Phil.

- grandiflora Lindl.

- viminea Spach.

Lopezia coronata Andr.

Oenothera biennis L.

- fruticosa L.

- Gigas Vries

- glauca Mchx. var. Fraseri Pursh

- missouriensis Sims.

- odorata Jacq. 
Oenothera pumila L.

- rosea Ait. (33)

\section{Oleaceae}

Jasminum odoratissimum L.

Fraxinus Ornus L.

\section{Orobanchaceae}

Aeginetia indica L.

Orobanche ramosa $\mathrm{L}$.

- speciosa DC.

\section{Oxalidaceae}

Biophytum dendroides $H$. B. et $K$.

- dormiens G. Don

- proliferum Edgew. et Hook.

- sensitivum DC.

Oxalis Acetosella L.

- cernua Thunb. (tub.)

- corniculata L. var. tropaeoloides Hook.

- Deppei Lodd. (tub.)

- lasiandra Zuce. (tub.)

- vespertilionis Zuce. (tub.)

\section{Papaveraceae}

Adlumia cirrhosa Rafin.

Argemone mexicana L.

- platyceras Link et Otto

Bocconia cordata Willd.

Chelidonium majus L.

Corydalis cheilanthifolia Hemsl.

- glauca Pursh

- lutea DC.

- nobilis Pers.

- ophiocarpa Hook. f. et Th.

- sibirica Pers.

Dicentra eximia Torr.

Eschscholtzia californica Cham.

Glaucium corniculatum Curt.

- flavum Crantz

Hunnemannia fumariifolia Sweet.
Hypecoum procumbens L.

Meconopsis cambrica Vig.

- racemosa Maxim.

Papaver alpinum L.

- Argemone L.

- caucasicum Bieb.

- dubium L.

- glaucum Boiss. et Hausskn.

- hybridum L.

- nudicaule L.

- orientale L.

- pavoninum F. et M.

- rupifragum Boiss.

- somniferum L.

_ - var. polycephalum

Roemeria rhoeadiflora Boiss.

- violacea Med.

Sanguinaria canadensis L.

Stylophorum diphyllum Nutt.

- Franchetianum Fedde

Pliytolaceaceae

Petiveria alliacea L.

Phytolacca decandra L.

Rivinia laevis L.

- tinctoria Ham.

Plantaginaceae

Plantago arenaria $\mathbf{W}$, et $\mathbf{K}$.

- Coronopus L.

- Cynops L. •

- lanceolata L.

- major L. (33)

- maritima L.

- media L.

- Psyllium L.

Plumbaginaceae

* Armeria plantaginea Willd. (Mainz)

Statice globulariifolia Desf.

- Limonium L. 


\section{Polemoniaceae}

Collomia coccinea Lehm.

- gilioides Benth.

- linearis Nutt.

Gilia achilleifolia Benth.

- capitata Sims.

- coronopifolia Pers.

- multicaulis Benth.

- squarrosa Hook. et Arn.

- tricolor Benth.

Phlox paniculata L.

Polemonium caeruleum L.

- filicinum Greene

- pauciflorum S. Wats.

\section{Polygonaceae}

Emex spinosa Campd.

Oxyria digyna Campd.

Polygonum Bistorta L.

- capitatum Buch.-Ham.

- Convolvulus L.

$\neq$

- cuspidatum S. et Z.

- Fagopyrum L.

- Hydropiper L.

- orientale L.

- Persicaria L.

- sachalinense F. Schm.

- tatarium L.

- virginianum $\mathrm{L}$.

- Weyrichii F. Schm.

Rumex Acetosa L.

- alpinus L.

- crispus L.

- domesticus Hartm.

- maritimus L.

- obtusifolius L.

- Patientia L.

- salicifolins Weinm.

- seutatus $\mathrm{L}$.

Portulacaceae
Anacampseros lanceolata Sw.

- rufescens DC.

- tomentosa Berg.

Calandrinia grandiflora Hook.

Portulaca grandiflora Hook.

- oleracea L.

- pusilla H. B. et K. (33)

Talinum patens Willd.

Primulaceae

Anagallis arvensis L.

- — f. coerulea

Androsace saxifragifolia Bunge

Dodecatheon Jeffreyi L.

Lysimachia punctata L.

- vulgaris L. var. dahurica R. Kuuth

Primula acaulis Jacq.

*_ Auricula L. (33)

- Beesiana Forrest

- Bulleyana Forrest

- cortusoides L.

- denticulata Sm.

- elatior Jacq.

- farinosa L.

- Florindae Ward.

- Giraldiana Pax

- involuerata Wall.

- japonica A. Gray

- kewensis hort.

- lichiangensis Forrest

- longiflora All. (33)

- luteola Rupr.

- officinalis Jaeq.

- pulverulenta Duth.

- rosea Royle

- vittata Bur. et Franch.

Samolus Valerandi L. (33)

\section{Ranunculaceae}

Aconitum Lyeoctonum L.

- paniculatum Lam.

- ranunculifolium Rehb. 
Actaca alba Mill.

- rubra Big.

- spicata L.

Anemone dichotoma L.

- multifida Poir.

- nemorosa $\mathrm{L}$.

- patens L.

- Pulsatilla L.

- rivularis Buch.-Ham.

- silvestris L.

- virginiana $\mathrm{L}$.

Aquilegia akitensis Huth.

- alpina L.

- canadensis L.

Caltha palustris L.

Clematis integrifolia $\mathrm{L}$.

- recta $\mathrm{L}$.

- Spooneri Rehd, et Wils.

- tangutica Korsh.

var. obtusiuscula Rehd. et Wils.

- Viticella L.

Delphinium Consolida L.

- grandiflorum L. var. ehinense Huth.

- nudicaule Torr. et Gray

- turkestanicum Huth.

Eranthis cilicica Schott. et Ky.

- hiemalis Salisb.

Helleborus foetidus L.

- niger L. var. macranthus Schiffn.

- purpurascens W. et K.

Leptopyrum fumarioides Rehb.

Myosurus minimus L.

Nigella damascena L.

- Garidella Spenn.

- hispanica L.

- integrifolia Rgl.

- orientalis L.

- sativa L.

Paeonia corallina Retz.

- officinalis $\mathbf{L}$.
Paeonia peregrina Mill.

- Veitchii Lynch

Ranunculus abortivus I.

- acer L.

- aconitifolius L.

- arvensis L.

- Cymballaria Pursh

- lanuginosus $\mathrm{L}$.

- sceleratus L.

Thalictrum angustifolium Jacq.

- aquilegifolium $\mathrm{L}$.

- glaucum Desf.

- minus $\mathrm{L}$.

- simplex L.

Trollius europaeus L.

* - - (Rhön)

- pumilus D. Don var. yunnanensis

Franch.

\section{Resedaceae}

Reseda alba L.

- crystallina Webb. et Bert.

- lutea L.

- Luteola L.

- odorata L.

\section{Rȟ́mnaceae}

Rhamnus cathartica L.

- dahurica Pall.

- Frangula L.

\section{Rosaceae}

Acaena myriophylla Lindl.

- pinnatifida Ruiz. et Pav.

- Sanguisorbae Vahl.

Agrimonia Eupatoria L.

Alchemilla alpina L. (33)

- pubescens Lam.

- vulgaris L.

Amelanchier rotundifolia Dum.-Court.

Aronia arbutifolia Spach

Aruneus silvester Kost. 
Chaenomeles japonica Lindl.

Cotoneaster Dammeri G. Schneid.

- horizontalis Dene.

Grataegus Grus galli L.

- nitida Sarg.

Dryas Drummondii Rich.

" octopetala L. (33)

Duchesnea indica Focke

Filipendula hexapetala Gilib.

- Ulmaria Maxim.

Fragaria vesca L.

Geum album Gmel.

- coccineum Sibth.

- montanum L.

- rivale L.

- strictum Ait.

- urbanum L.

Gillenia trifoliata Moench

Holodiseus discolor Aschs, et Graebn. Mespilus germanica L.

Physocarpus opulifolius Rafin.

Potentilla argentea L.

- atrosanguinea Lodd.

- aurea L.

- canescens Bess.

- fulgens Willd.

- Hippiana Lehm.

- megalantha Tak.

- nepalensis Hook.

- norvegica $L$.

- recta $\mathrm{L}$.

- - var. corymbosa A. et G.

- reptans L.

- rupestris $\mathbf{L}$.

- supina L.

Prunus pumila L.

- spinosa L.

Pyracantha coceinea Röm.

Rhodothypus kerrioides S. et Z.
Rosa dahurica Pall.

- gymnocarpa Nutt.

- Moyesii Hemsl. et Wils.

- setigera Rich.

Sanguisorba minor Scop.

- officinalis L.

- tenuifolia Fisch. var. albiflora

Sibbaldia procumbens L.

Sorbaria sorbifolia A. Br.

Sorbus domestica L.

- torminalis Crantz

Stranvaesia Davidiana Dene.

\section{Rubiaceae}

Asperula arvensis L.

* cynanchica L. (Mainz)

- hexaphylla All.

- odorata L.

- orientalis Boiss. et Reut.

- tinctoria L.

Crucianella stylosa Trin.

Galium Aparine L. (33)

- Molluga L.

- parisiense L.

- purpureum L.

- saccharatum All. (33)

- silvaticum L.

- verum $\mathrm{L}$.

Rubia tinctorum L.

Sherardia arvensis L.

Rutaceae

Dictamnus albus L.

Erythrochiton brasiliense N. et M.

Ptelea trifoliata L.

Ruta graveolens L.

Sapindaceae

Koelreutera paniculata Laxm.

Saxifragaceae

Astilbe Davidii Henry (33) 
Bergenia crassifolia Engl.

Boykinia rotundifolia Parry

Francoa sonchifolia Cav.

Heuchera bracteata Ser.

- sanguinea Englm.

Peltiphyllum peltatum Engl.

Rodgersia podophylla A. Gray

- tabularis Kom.

Saxifraga Aizoon Jacq.

- altissima Kern.

- caespitosa L.

- Cotyledon L.

- crustata Vest.

- geranioides L.

- granulata L.

- irrigua M. Bieb.

- Portae Stein

- rotundifolia $\mathrm{L}$.

Tellima grandiflora R. Br.

Tiarella polyphylla D. Don

\section{Serophulariaceae}

Antirrhinum molle L.

- Orontium L.

Galceolaria chelidonioides H. B. et K.

- pinnata L.

- scabiosaefolia Sims.

Chaenorrhinum minus Lange

- - var. praetermissum Cos.

Chaenostoma foetidum Benth.

Chelone obliqua L.

Collinsia bicolor Benth.

Diascia Barberae Hook. f.

Digitalis ambigua Murr.

- ferruginea $L$.

- lanata Ehrh.

- lutea L.

- purpurea L.

Erinus alpinus L.

Gratiola officinalis $L$.
Hebenstreitia dentata L.

Linaria genistifolia Mill.

- origanifolia DC.

- striata DC.

- Tournefortii Lge.

Lyperia violacea Benth.

Maurandia Purpusii T. S. Brand

Mimulus cardinalis Dougl.

- luteus L.

- moschatus Dougl. (33)

- pilosiusculus $H$. B. et K.

Nemesia chamaedryfolia Vent.

- foetens Vent.

- pubescens Benth.

- strumosa Benth.

Pentstemon barbatus Nutt.

- campanulatus Willd.

- glaber Pursh

-- hirsutus Willd.

Scrophularia alata Gilib.

- canina L.

- lateriflora Trautv.

- nodosa $\mathbf{L}$.

- Scopolii Hoppe (33)

- vernalis $\mathbf{L}$.

Selago muralis Benth. et Hook.

Sphaenandra viscosa Benth.

Teedia lucida Rud.

Tetranema mexicanum Benth.

Verbascum Blattaria L. (33)

- longifolium Ten. (33)

- nigrum L.

- olympicum Boiss.

- phlomoides L.

- phoeniceum L. (33)

Veronica austriaca $\mathbf{L}$.

- Chamaedrys L.

- fruticans Jaeq.

- fruticulosa L.

- longifolia L. 
Veronica officinalis L.

- prostrata L. (33)

- Schmidtiana Rgl.

-- spicata L.

- spuria L.

\section{Solanaceae}

Atropa Belladonna L.

Browallia viscosa $H$. B. et $\mathrm{K}$.

Capsicum annuum $\mathbf{L}$.

Datura inermis Jacq.

- Metel L.

- Stramonium L.

- Tatula L.

Hyoseyamus niger $\mathrm{L}$.

- - var. pallidus W. et K.

Nicandra physaloides Gaertn.

Nicotiana Langsdorffii Weinm.

- longiflora Cav.

- nudicaulis S. Wats.

- rustica L.

- Tabacum L.

- - var. macrophylla Schrank

Nierembergia frutescens Dur.

Petunia nyctaginiflora Juss.

- parviflora Juss.

- violacea Lindl.

Schizanthus pinnatus R. et $P$.

Solanum Duleamara L.

- heterodoxum Dun.

- Lyeopersicum L. var. cerasiforme

- - - Humboldtii

- - - pyriforme

- macrocarpum L.

- nigrum L.

- sisymbriifolium Lam.

- villosum Lam.

Withania somnifera Dun.
Staphyleaceae

Siaphylea colchica Scop.

- pinnata $\mathbf{L}$.

\section{Sterculiaceae}

Hermanuia alnifolia L. (33)

Styracaceae

Halesia tetraptera L.

Symplocaceae

Symplocos martinicensis Jucq.

Thymelaeaceae

Daphne Mezereum L.

\section{Tiliaceae}

Tilia petiolaris DC.

- tomentosa Moench

Tropaeolaceae

Tropaeolum majus $\mathrm{L}$.

- - - var. nanum hort.

- minus L.

- peltophorum Benth.

\section{Ulmaceae}

Celtis australis L.

\section{Umbelliferae}

Anethum graveolens L.

Anthrisens Cerefolium Hoffm.

- silvestris Hoffm.

Apium tenuifolium Then.

Arehangelica officinalis Hoffm.

Astrantia major L.

Bupleurum Candollii Wall.

- faleatum L.

- filicaule Brot

- rotundifolium L. 
Carum Carvi L.

Caucalis daucoides $\mathrm{L}$.

Genolophium Fischeri K. Koch

Chaerophyllum aromaticum L.

Cicuta virosa $\mathrm{L}$.

Conium maculatum L.

Coriandrum sativum L.

Daucus pulcherrimus Koch

Eryngium amethystinum L.

* - campestre L. (Mainz)

- planum L.

Falcaria vulgaris Bernh.

Foeniculum vulgare Mill.

Hacquetia Epipactis DC.

Heracleum Lehmannianum Bge.

- Mantegazzianum S. et Z.

- Sphondylium L.

- villosum Fisch.

Laserpitium pruthenicum L.

Levisticum officinale Koch

Meum athamanticum Jacq.

Myrrhis odorata Scop.

Oenanthe Lachenalii Gmel.

Orlaya grandiflora Hoffm.

Peucedanum officinale $L$.

- Oreoselinum Moench

- Ostruthium Koch

Pimpinella Anisum L.

- magna L.

- peregrina L. (33)

Sanicula europaea L.

Seandix macrorynchia C. A. Mey.

- Pecten Veneris L.
Selinum Carvifolia L.

Seseli glancum Iacq.

Siler trilobum Scop.

Sium angustifolium $\mathrm{L}$.

- Sisarum L.

Trachymene pilosa Sm.

\section{Urticaceae}

Bowlesia tenera Spreng.

Laportea canadensis Gaud.

- moroides Wedd.

Parietaria officinalis $\mathrm{L}$.

Pilea muscosa Lindl.

- Spruceana Wedd.

Urera baccifera Gaud.

Urtica cannabina L.

- dioica $L$.

- Dodartii L.

- pilulifera L.

- urens $\mathbf{L}$.

Valerianaceae

Centranthus ruber DC.

Fedia Cornucopiae DC.

Valeriana officinalis L.

Valerianella coronata DC.

- dentata Pall.

- eriocarpa Desv.

- rimosa Bastard

Verbenaceae

Callicarpa Giraldiana Hesse

- japonica Thunb.

- longifolia Lam.

Verbena officinalis L.

- tenera Spr. 
Violaceae

Hymenanthera crassifolia Hook.

Viola bosniaca Form.

- canadensis L.

- collina Bess.

- lutea Huds.
Viola mirabilis L.

- Riviniana Rehb.

- tricolor L. f. arvensis

Zygophyllaceae

Tribulus orientalis Kern. (33)

Signum * indicat semina e plantis spontaneis lecta.

$(33)=$ semina anno 1933 lecta.

Für den Botanischen Garten der Universität:

\section{Professor Dr. F. Laibach}

Direktor
Für den Haupt-Schulgarten der Stadt:

Bromme

Gartenbaudirektor

Kiehne

Gartenoberinspektor 


\section{Frankfurt am Main}

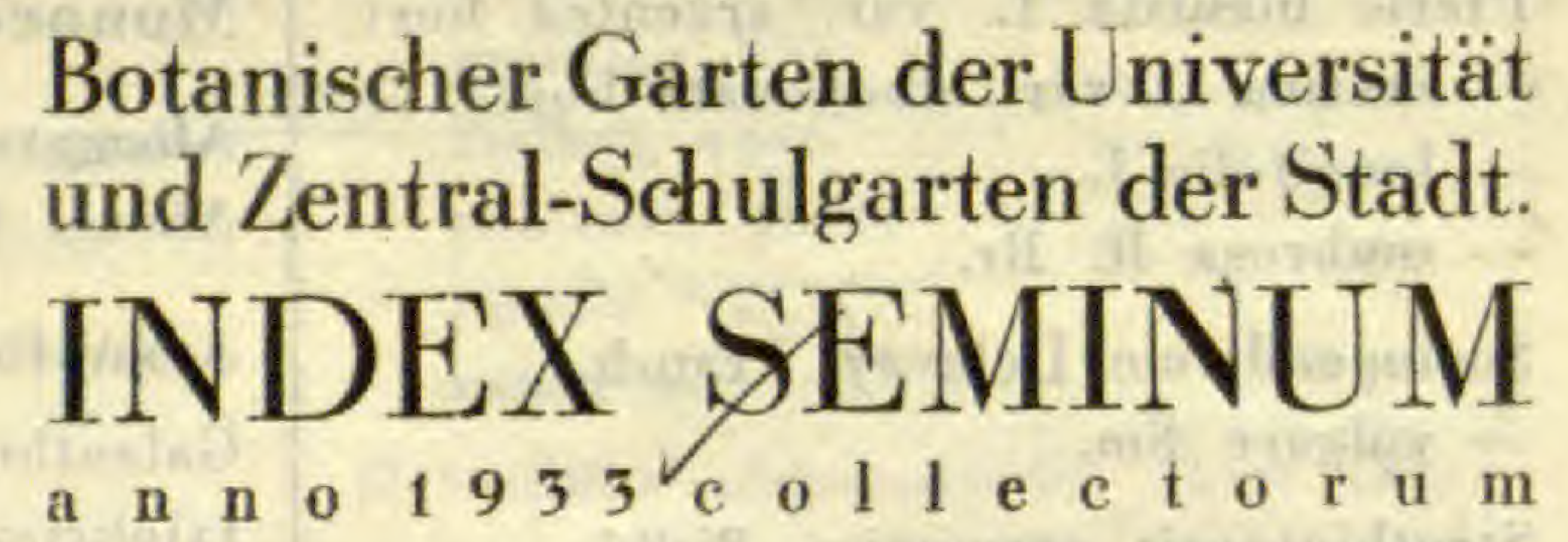

Desideratainscribenda sunt: An die Direktion des Botanischen Gartens, Frankfurt-M.

\section{Pteridophyta}

Cyatheaceae

Dicksonia antaretica Lab.

\section{Marattiaceae}

Marattia alata Sw.

\section{Parkeriaceae}

Ceratopteris thalietroides Brongn.

Polypodiaceae

Adiantum caudatum L.

- hispidulum Sw.

- reniforme $\mathbf{L}$.

- trapeziforme $\mathbf{L}$.

Aspidium Filix mas Sw.

- melanocaulon BI.

- spinulosum Sw.

Asplenium Belangeri Kze.

- bulbiferum Forst.

- Nidus L.

var. Fabianum Bak.

Athyrium Filix femina Roth

Blechnum brasiliense Desv.

- longifolium H. B. et K.

$$
\text { var. fraxineum Bak. }
$$

- Moorei C. Chr.

- Spicant Sm.

Davallia bullata Wall.

- Tyermannii Bak.
Deparia Moorei Hook.

Diplazium Petersenii Christ

- proliferum Thouars

- Shepherdii Link

Doodia aspera $\mathrm{R}$. Br.

Dryopteris parasitica Kze.

- patens Kze. var. macroura Kaulf.

- Phegopteris C. Chr.

- Poiteana Urb.

- Sieboldii Kze.

Fadyenia prolifera Hook.

Gymnogramme calomelanos Kaulf. var. Massonii Link

- tatarica Desv.

Hemionitis ariifolia Moore

Microlepia speluncae Moore

Nephrolepis acuminata Kuhn

Niphobolus adnascens Kaulf.

- Lingua I. Sm.

Pellaea rotundifolia Hook.

- viridis Prantl

Platycerium alcicorne Desv.

- divergens Desv.

- Veitchii hort.

- Willinckii Moore

Polypodium aureum $\mathbf{L}$.

vax-eristatum hort.

- irioides Lam

- Meyenianum Schotes C E \& I :

- vulgare $\mathrm{f}$. 
Polystichum pungens Presl

Pteris biaurita L. var. argentea hort.

- eretica L. var. alboliueata hort.

- longifolia L.

- umbrosa R. Br.

Scolopendrium Delavayi Franch.

- vulgare Sm.

Struthiopteris germanica Willd.

Schizaeaceae

Aneimia densa Link

- Dregeana Kze.

- fraxinifolia Raddi

- Mandiocana Raddi

- rotundifolia Schrad.

Lygodium japonicum Sw.

\section{Selaginellaceae}

Selaginella Emmeliana van Geert

- inaequalifolia Spring.

- Kranssiana A. Br.

- pilifera A. Br.

- stenophylla A. Br.

\section{Gymnospermae}

\section{Cupressaceae}

Chamaecyparis pisifera Endl.

\section{Pinaceae}

Larix decidna Mill.

- leptolepis Gord.

- sibirica Ledeb.

Picea canadensis B.S.P.

Pinus excelsa Wall.

- nigra Arn.

- Strobus L.

Pseudotsuga taxifolia Britt.

Tsuga canadensis Carr.

\section{Taxaceae}

Taxus baceata L.

- - var. adpressa Carr.

\section{Angiosperimae \\ Monocotyledoneae}

\section{Alismataceae \\ Alisma Plantago L.}

\section{Amaryllidaceae}

Galanthus byzantinus Bak.

Ixiolirion Pallasii Fisch. et Mey.

Leucojum vernum $\mathbf{L}$.

Narcissus Bulbocodium L.

Zephyranthes Atamaseo Herb

\section{Araceae}

Arum maculatum L.

Calla palustris $\mathrm{L}$.

Remusatia vivipara Schott

Xanthosoma Maximilianum Schott

\section{Bromeliaceae}

Acanthostachys strobilacea Link

Aechmea bromeliifolia Bak.

Caraguata lingulata Lindl.

Dyckia remotiflora Ott. et Dietr.

Guzmannia tricolor Ruiz. et Pav.

Tillandsia Saundersii C. Koch

\section{Commelinaceae}

Commelina coelestis Willd.

- clandestina Mart.

- communis L.

- dianthifolia Red.

Palisota Barteri Hook. f.

Rhoeo discolor Hance

Tinantia Fugax Scheidw.

\section{var erecta Drum.}

Tradescantia virginiana $\mathrm{L}$.

Cyperaceae

Carex cyperoides L.

- Graygii Car.

- lycopodioides De.

- muricola L.

- vulpina $\mathrm{L}$. 
Cyperus adenophorus Schrad.

- alternifolius $\mathbf{L}$.

- esculentus L. (bulb.)

\section{Gramineae}

Aegilops erassa Boiss

- ovata L.

Ammophila arenaria Link

Andropogon Ischaemon L.

Apera Spica venti Beauv.

Asperella Hystrix Humb.

Aristida capillacea Lam.

Avena brevis Roth (32)

Brachypodium distachyum Beauv.

- pinnatum Beauv.

- silvaticum R. et Sch.

Briza maxima L. (32)

Bromus asper Murr.

- commutatus Schrad.

- erectus Huds. (32)

- Kalmii A. Gray

- madritensis L.

- mollis L.

- patulus M. et K.

- pendulinus Schrad.

- secalinus L.

- unioloides H. B. et K.

Catabrosa humilis Trin.

Cenchrus echinatus L.

- montanus Nees

- tribuloides L.

Chaeturus fasciculatus Link

Coix Lacryma Jobis L.

Dactylis glomerata L. (32)

- - var. hispanica

Deschampsia caespitosa Beanv.

- flexuosa Trin.

Diarrhena americana Beauv.

Elymus curvatus Piper

- europaeus $\mathbf{L}$.

Eragrostis major Host. (32)

- nigra Nees

- pilosa Beauv.
Festuca arundinacea L.

- frigida Hack.

- gigantea Vill.

- Halleri Auct.

- pratensis Huds.

- pumila Vill. (32)

- rubra L.

Heleochloa alopecuroides Host (32)

Hordeum hexastichum L.

- tetrastichum L. var. nigrum

Ischaemum ciliare Retz

Lamarkia aurea Moench

Lolium italicum A. Br.

- remotum Schrank

- temulentum L.

Melica ciliata L. (32)

Milium effusum L. (32)

Molinia coerulea Moench (32)

Muehlenbergia glomerata Trin. (32)

Panicum capillare L. (32)

- Crus galli L.

- sanguinale $\mathbf{L}$.

Paspalum racemosum Lam.

Phalaris canariensis L.

- minor Retz

- paradoxa L.

Phleum asperum Jacq.

- Boehmeri Wib.

- Michelii All.

Poa alpina $\mathbf{L}$.

- compressa L.

- nemoralis L.

Polypogon interruptus H. B. et K.

- maritimus Willd.

- monspeliensis Desf.

Setaria germanica Beauv.

- glauca Beauv.

- verticillata Beauv.

- viridis Beauv. 
Sorghum vulgare Pers. var nigrum - - - saccharatum

Stipa Aristella L.

- capillata L.

- sibirica Lam.

- tortilis Desf.

Tragus racemosus Desf.

Tricholaena grandiflora Hochst.

Triticum dicoceum Schrank (32)

- monococeum L. (32)

- Spelta L.

Weingaertneria canescens Bernh. Zea Mays L.

- - var. oriziformis

\section{Iridaceae}

Aristea Ecklonii Bak. (32)

Crocus Fleischerei Gay

- nudiflorus Sm.

- pulchellus Herb.

- speciosus M. Bieb.

- Tomasinianus Herb.

- versicolor Ker.

- zonatus Gay

Iris bucharica Foster

- dichotoma Pall.

- Hoogiana Dykes

- Kaempferi Sieb.

- notha M. Bieb.

- pallida Lam.

- Pseudacorus L.

- pumila L.

- sibirica L.

- versicolor $\mathbf{L}$.

Lapeyrousea cruenta Benth.

Marica coerulea Ker.-Gawl.

Sisyrinchium striatum Sm.

\section{Juneaceae}

Juncus bufomus L.

- lampocarpus Ehrh.
Luzula albida De.

- nivea De.

\section{Liliaceae}

Agapanthus umbellatus L'Hérit.

Allium azureum Ledeb.

- fallax Schult.

- flavum L.

- fistulosum L. (32)

- karataviense Rgl.

- Moly L.

- oleraceum L.

- Ostrowskianum Rgl.

- rotundum $\mathbf{L}$.

- sativum L. (bulb.)

- Schoenoprasum L.

- stipitatum Rgl.

- Victorialis L.

Anthericum Liliago L.

Asphodeline lutea Rchb.

Asphodelus Villarsii Verl.

Bowiea volubilis Harv. (32)

Bulbine annua Willd.

Bulbocodium vernum $L$.

Camassia esculenta Lindl.

Chionodoxa sardensis Barr. et Sugd.

Chlorophytum amaniense Engl.

- comosum Bak. var. variegatum

- ukambense Bak.

Colchicum Bornmuelleri Freyn.

Eremurus himalaicus Bak.

- Kaufmannii Rgl.

- robustus $\mathrm{Rgl}$.

Fritillaria Meleagris L.

- imperialis $\mathbf{L}$.

Galtonia candicans Dene.

Haemanthus albiflos Jacq.

Hosta coerulea Tratt. (32)

- Sieboldiana Engl.

Kniphofia aloides Moench 
Lilium croceum Chaix

- Henryi Bak.

- Martagon L.

- regale Wils.

- superbum L.

Majanthemum bifolium Schmidt

Muscari botryoides Mill.

- comosum Mill.

- racemosum Mill.

Nothoscordum fragrans Kunth

Ornithogalum narbonense L.

Polygonatum latifolium Desf.

- multiflorum All.

- officinale All.

Puschkinia scilloides Adams

Scilla nonscripta Hoffm. et Link (32)

- sibirica Andr. (32)

*Tofielda palustris Huds.

Tricyrtis macropoda Miq.

Tulipa silvestris $\mathbf{L}$.

- Sprengeri Bak.

Urginea maritima Bak. (32)

Veratrum nigrum $\mathbf{L}$.

Scheuchzeriaceae

Triglochin maritima $\mathbf{L}$.

- palustris $\mathbf{L}$.

Typhaceae

Typha latifolia L.

- minima Funk

\section{Taccaceae}

Tacea maerantha Jaeq.

\section{Zingiberaceae}

Globba Schomburgkii Hook. f.

\section{Dicotyledoneae}

\section{Acanthaceae}

Acanthus longifolius Host
Hemigraphis primulaefolia Vill.

Strobilanthes roseus Nees

Thunbergia alata Boj.

Thyrsacanthus rutilans Planch.

Aceraceae

Acer campestre L.

- cappadocicum Gled.

- circinatum Pursh

- Ginnala Maxim.

- macrophyllum Pursh

- monspessulanum $\mathrm{L}$.

- tataricum L.

\section{Aizoaceae}

Mesembrianthemum cordifolium L.

- cristallinum L. (32)

- linguiforme $\mathbf{L}$.

- pinnatifidum L.

- pomeridianum $\mathbf{L}$.

- pyropaeum Haw.

Tetragonia echinata Ait.

- expansa Ait.

\section{Amarantaceae}

Achyranthes aspera L.

Alternanthera sessilis R. Br. (32)

Amarantus Blitum L.

- caudatus L.

- chlorostachys Willd.

- flavus L.

- paniculatus $\mathbf{L}$.

Froelichia floridana Moq.

Gomphrena globosa L. (32)

Telanthera polygonoides Moq. (32)

Anacardiaceae

Rhus vernicifera DC.

\section{Aquifoliaceae}

Ilex Aquifolium L.

- glabra Gray 
Araliaceae

Aralia spinosa L.

\section{Aristolochiaceae}

Aristolochia macrophylla Lam.

Asarum europaeum L.

Asclepiadaceae

Asclepias Cornuti Decne.

- curassavica L. (32)

- incarnata $L$.

Ceropegia debilis N. E. Br. (bulb.)

- Woodii Schlecht. (bulb.)

Dischidia pectenoides Pears.

Stapelia grandiflora Mass.

Vincetoxicum officinale Moench

\section{Balsaminaceae}

Impatiens Balfourii Hook. f.

- Balsamina L.

- biflora Walt.

- Holstii Engl. et Warb.

- Noli tangere L.

- parviflora DC.

- Roylei Walp.

\section{Basellaceae}

Boussingaultia baselloides

H. B. et $\mathrm{K}$. (rhiz.)

\section{Begoniaceae}

Begonia Schmidteana Rgl.

\section{Berberidaceae}

Berberis Thunbergii DC.

- Veitchii C. Schneid.

- vulgaris $L$.

- Wilsonae Hemsl. et Wils.

Podophyllum Emodi Wall.

\section{Betulaceae}

Betula lenta L.

\section{Bignoniaceae}

Gatalpa bignonioides Walt.

- ovata G. Don

Eccremocarpus scaber R. et P. (32)

Incarvillea compacta Maxim.

- Delavayi Bur. et Franch.

var. brevipes

- Olgae Rgl.

\section{Borraginaceae}

Anchusa capensis Thuill.

- officinalis $\mathbf{L}$.

- sempervirens L. (32)

Cerinthe major L.

- minor $\mathbf{L}$.

Cynoglossum officinale $\mathbf{L}$.

- pictum Ait.

Echinospermum Lappula Lehm.

Echium ereticum L.

- plantagineum L.

- vulgare $\mathbf{L}$.

Eritrichium strictum DC.

Heliotropium anchusaefolium Poir.

- europaeum L.

Lindelofia longifolia Guerke

Lithospermum arvense $\mathbf{L}$.

- officinale L.

- purpureo-coeruleum $\mathbf{L}$.

Lycopsis arvensis $\mathbf{L}$.

Nonnea rosea Link

Omphalodes linifolia Moench

\section{Cactaceae}

Opuntia camanchica Englm. et Bigl.

- - - var. albispina

- missouriensis DC.

\section{Campanulaceae}

Adenophora latifolia Fisch.

- liliifolia Ledeb.

- stylosa Fisch.

- verticillata Fisch. var. typica 
Campanula alliarïifolia Willd.

- barbata L.

- carpatica Jacq. (32)

- Cervicaria L.

- drabifolia Sibth. et Sm.

- garganica Ten.

- glomerata L. var. dahurica

- lactiflora Bieb.

- latifolia L.

- patula L.

- persicifolia L. var. macrantha

- Portenschlagiana R. et Sch.

- pusilla Haenke

- Raddeana Trautv.

- ramosissima Sibth. et Sm.

- rapunculoides $\mathrm{L}$.

- rotundifolia $\mathbf{L}$.

- Scheuchzeri Vill.

- sibirica L.

- Trachelium L.

- turbinata Schott.

- velutina Desf.

Codonopsis clematidea G. B. Clarcke

- ussuriensis Hemsl.

Downingia elegans Torr.

Hedraeanthus graminifolius A. DC.

Jasione montana L.

- perennis Lam.

Lobelia Cliffortiana L.

- inflata $L$.

- syphilitica $\mathbf{L}$.

Phyteuma canescens W. et K.

- nigrum Schmidt

- orbiculare L.

- Scheuchzeri All.

- spicatum L.

Platyeodon grandiflorum A. DC.

- - - var. album

Specularia falcata A. DC.

- pentagonia A. DC.

- perfoliata A. DC.

- Speculum A. DC.
Symphyandra Hofmannii Pant.

Wahlenbergia hederacea Rchb.

\section{Capparidaceae}

Cleome monophylla L.

- pungens Willd.

- - var. albiflora

- violacea $\mathrm{L}$.

Dactylaena micrantha Schrad.

\section{Caprifoliaceae}

Lonicera coerulea L.

Sambucus racemosa L.

Viburnum Opulus L.

- rhytidophyllum HemsI.

\section{Caryophyllaceae}

Agrostemma Githago L.

Alsine tenuifolia Wahl.

Anychia dichotoma Mchx.

Arenaria graminifolia Schrad.

- rubra $L$.

- serpyllifolia L. (32)

Cerastium chlorifolium F. et M.

- dichotomum L.

Corrigiola littoralis $\mathbf{L}$.

Cucubalus baccifer L.

Dianthus Armeria L. (32)

- banaticus Heuff.

- Garthusianorum L.

- - var. atrirubens

- negleetus Lois.

- pelviformis Heuff.

- plumarius L.

- Seguierii Vill.

- spiculifolius Schur.

- superbus L.

Drymaria cordata Willd.

Gypsophila altissima L.

- paniculata $\mathbf{L}$.

- repens $\mathbf{L}$. 
Heliosperma alpestre Rchb.

Herniaria incana Lam.

Lychnis alpina L.

- chalcedonica L.

- Coronaria Desr.

- Flos cuculi L.

- Flos Jovis Desr.

- Haageana hort.

- Viscaria L.

Melandryum album Garcke

- rubrum Garcke

Moehringia trinerva Clairv.

Paronychia serpyllifolia DC.

Polycarpon tetraphyllum L.

Sagina procumbens L.

Saponaria calabrica Guss.

- cerastioides F, et M.

- officinalis L.

- persica Boiss.

Scleranthus aunuus L.

*Silene acaulis $\mathbf{L}$.

- Armeria L.

- compacta Fisch. (32)

- dichotoma Ehrh.

- echinata Otth.

- fuscata Link

- gallica L. (32)

- italica Pers.

- linicola Gmel.

- mellifera Boiss. et Reut.

- multicaulis Guss.

- Muscipula L.

- nutans L.

- rubella L.

- Schafta Gmel.

- vulgaris Garcke

Spergula arvensis L.

- pentandra L.

Tunica prolifera Scop.

- Saxifraga Scop.

- velutina Fisch. et Mey.
Vaccaria pyramidata Med.

\section{Celastraceae}

Evonymus alata Rgl.

- europaea L.

- latifolia Scop.

- radicans Miq.

Chenopodiaceae

Atriplex hortense L.

- patulum L.

Axyris amarantoides L.

Chenopodium album L.

- ambrosioides L.

- - var. anthelminticum

- Bonus Henricus L.

- Botrys L.

- polyspermum L.

- Quinoa L.

- rubrum L.

Hablitzia tamnoides Bieb.

Monolepis trifida Schrad.

Salsola Kali L.

\section{Cistaceae}

*Helianthemum Chamaecistus Mill.

- guttatum Mill.

\section{Clethraceae}

Glethra acuminata Michx.

- alnifolia L.

\section{Compositae}

Achillea Glavennae L. (32)

- Millefolium L.

- ptarmica L.

- sibirica Ledeb.

"Adenostyles albifrons Rehb.

Anacyclus of ficinarum Hayne

Antennaria dioica Gaertn.

Anthemis tinctoria $\mathbf{L}$.

Aretotis grandis Thunb. 
Arnica Chamissonis Less.

- montana L.

- sachalinensis A. Gray

Artemisia Absinthium L.

- annua $\mathrm{L}$.

- sacrorum Ledeb. var. viridis

- vulgaris L.

Aster alpinus L.

- caucasicus Willd.

- macrophyllus $L$.

- Novi Belgii Lam.

- subcoeruleus L. Moore

- Tripolium L.

- umbellatus MilI.

Baeria gracilis Gray

Bidens leucanthus Willd.

- tripartitus L.

Brachycome iberidifolia Benth.

Buphthalmum salicifolium L.

- speciosum Schreb.

Calendula arvensis L.

- officinalis L.

Callistephus chinensis Nees "Helvetia“

Carduus acanthoides L. (32)

- crispus L.

- nutans L.

- stenolepis Benth. (32)

Carlina acaulis L.

- longifolia Rchb.

- vulgaris L.

Carthamus lanatus L.

- tinetorius L.

Centaurea Crocodylium L.

- involucrata Desf.

- Jacea L.

- Lippii L.

- montana L.

- nigra $\mathbf{L}$.

- Scabiosa L.

- sulphurea Willd.
Cephalophora aromatica Schrad.

Charieis heterophylla Cass.

Chrysanthemum cinerariifolium Trev.

- corymbosum L.

- Leucanthemum L.

- macrophyllum W. et K.

- viscosum Desf. (32)

- vulgare Bernh.

Chrysocoma Coma aurea L.

Cirsium eriophorum Scop.

- oleraceum Scop. (32)

- pannonicum Gaud.

Cnicus benedictus L.

- syriacus Roth. (32)

Coreopsis Atkinsoniana Dougl.

- coronata Hook. (32)

- Drummondii Torr. et Gray

- grandiflora Nutt. (32)

- lanceolata L.

- tripteris $\mathbf{L}$.

Cosmos atrisanguineus Hook.

Cotula coronopifolia L.

- turbinata L.

Crepis aurea Cass.

- blattarioides Vill.

- montana Tausch.

- rubra L.

- sibirica L.

- taraxacifolia Thuil.

Crupina paueiflora Hoffm. et Link

Cryptostemma calendulaceum R. Br.

Cynara Scolymus L.

Dahlia Merckii Lehm.

Doronicum Pardalianches L.

Echinops sphaerocephalus L.

Emilia flammea Cass.

Erigeron acer L.

- speciosus DC.

- Villarsii Bell. 
Eupatorium cannabinum L.

- perfoliatum L.

- urticifolium Rchb.

Felicia fragilis Cass.

Filago germanica L.

Gaillardia Amblyodon A. Gray

- hybrida hort.

Gazania longiscapa DC.

Gnaphalium silvaticum L.

Grindelia squarrosa Pursh

Hedypnois eretica Willd.

Helenium Hoopesii Gray

- puberulum DC.

Helianthus annuus L. var. uniflorus

Helichrysum arenarium DC.

- bracteatum Willd. var. monstrosum

- foetidum Moench

- orientale Gaertn.

Heliopsis helianthoides Sweet

Helipterum Manglesii F. v. Muell.

- roseum Benth.

Hieracium amplexicaule L.

- aurantiacum L.

- Bornmuelleri Freyn.

- bupleuroides Gmel.

- humile Jacq.

- laevigatum Willd.

- murorum L.

- silvestre Tausch.

- vulgatum Vries

Hypochoeris glabra L.

Inula Conyza DC.

- glandulosa Puschk.

- Helenium L.

Koelpinia linearis Pall. (32)

Lactuca altissima Bieb.

- muralis Less.

- perennis $\mathbf{L}$.

- Scariola L.

- virosa $\mathbf{L}$.
Lagascea mollis Cav.

Lappa major Gaertn.

- minor DC.

- tomentosa All.

Lasthenia glabrata Lindl.

Laya elegans Torr. et Gray

Leontopodium alpinum $\mathrm{L}$.

Leptosyne maritima A. Gray

Liatris spicata Willd.

Ligularia macrophylla DC.

Madia sativa Mol.

Matricaria Chamomilla L.

Microseris Lindleyi A. Gray

Moscharia pinnatifida R. et P. (32)

Mulgedium Plumieri DC.

- sibiricum Less.

Onopordon Acanthium L.

Palafoxia Hookeriana Torr. et Gray

Picridium tingitanum Desf. (32)

Picris echioides L. (32)

Pulicaria dysenterica Gaertn.

Relhania sessiliflora Thunb.

Rhagadiolus stellatus Willd.

Rodigia commutata Spreng (32)

Rudbeckia amplexicaulis Vahl.

- bicolor Nutt.

- laciniata L.

- purpurea L.

- speciosa Wend.

Sanvitalia procumbens Lam.

Scorzonera parviflora Jacq.

Senecio clivorum Maxim.

- Fuchsii Gmel.

- Veitchianus Hemsl.

Serratula tinctoria $\mathbf{L}$.

Siegesbeckia orientalis L.

Silphium perfoliatum L. 
Silybum Marianum Gaertn.

Solidago Virg-aurea L.

Sonchus palustris L.

Stevia purpurea Pers.

- serrata Cav.

Taraxacum officinale Web. (32)

Thelesperma Burridgeanum hort.

Tolpis barbata Gaertn.

Tragopogon major Jacq.

- porrifolius L. (32)

Tridax trilobata Hemsl.

Troximon gracilens A. Gray (32)

Ursinia speciosa DC.

Verbesina encelioides Benth. et Hook

Xanthium spinosum L.

- Strumarium L.

Xeranthemum inapertum Mill.

Zinnia pauciflora L. (32)

- verticillata Andr. (32)

\section{Convolvulaceae}

Convolvulus elongatus Willd.

- farinosus L.

- pentapetaloides $\mathbf{L}$.

- tricolor L.

- undulatus Cav.

Cuscuta Epilinum Weihe

- europaea L.

Ipomoea purpurea Roth (32)

Mina lobata Llaw. et Lex.

Quamoclit coccinea Moench

\section{Cornaceae}

Cornus Baileyi Coult. et Ev.

- mas L.

\section{Crassulaceae}

Aichryson dichotomum Webb. et Bert.

Kalanchoe glaucescens Britt. (32)
Sedum acre L.

- Aizoon L.

- kamtschaticum Fisch. et Mey.

- maximum Sut.

- purpureum Link

- reflexum $\mathrm{L}$.

- sediforme C. Pau

- spurium M. B.

Sempervivum tectorum L.

Sinocrassula yunnanensis Berg.

\section{Cruciferae}

Aethionema cappadocicum Spreng.

Alliaria officinalis Andr. (32)

Alyssum calycinum L.

- maritimum Lam.

- petraeum Ard.

- saxatile L.

Arabis alpina L.

- arenosa Scop.

- bellidifolia Jacq.

- hirsuta Scop.

- perfoliata Lam.

- procurrens W. et K. (32)

- serpyllifolia Vill. var. nivalis

- Turrita L.

Barbaraea vulgaris $\mathrm{R}$. Br.

Biscutella didyma $\mathrm{L}$.

*- laevigata L.

Brassica Napus L.

- nigra Koch

- oleracea L. (32)

Braya supina Koch

Camelina sativa Crantz

Capsella grandiflora Boiss.

- Viguierii Blar.

Cardamine hirsuta L. (32)

- pratensis L. (32)

Chorispora tenella DC.

Coronopus procumbens Gilib.

- Ruellii All. 
Crambe abyssinica Hochst.

- cordifolia Stev.

— - var. palmatifida

- koktebelica Busch

- maritima L.

Diplotaxus tenuifolia DC.

Draba Aizoon Wahl.

- repens Bieb. (32)

Erysimum cheiranthoides L.

- Perowskianum F. et M.

Hesperis fragrans Fisch.

- matronalis L.

Iberis amara $\mathrm{L}$.

- Lagascana DC.

- sempervirens L.

Isatis tinctoria $\mathbf{L}$.

Lepidium Draba L.

- latifolium L.

- sativum L.

- virginicum $\mathrm{L}$.

Lunaria biennis Moench

- rediviva $\mathrm{L}$.

Malcomia chia DG.

- maritima Ait.

Matthiola bicornis DG.

- tricuspidata R. Br.

Neslea paniculata Desv.

Peltaria alliacea Jacq.

Raphanus sativus L. var. oleifer

Rapistrum rugosum All.

Ricotia Lunaria DC.

Schievereckia podolica Andr.

Sinapis alba $\mathrm{L}$.

- arvensis L.

Sisymbrium officinale Scop.

- strictissimum $\mathbf{L}$.

Turritis glabra L.

Vesicaria utriculata Lam.
Cucurbitaceae

Bryonia dioica Jaeq.

Gucumis myriocarpus Naud.

Cueurbita maxima Duch.

var turbaniformis

Cyclanthera explodens Naud.

- pedata Schrad.

Ecballium Elaterium A. Rch.

Melothria pendula L.

Sicyos angutala $\mathbf{L}$.

Thladiantha dubia Bunge

Datiscaceae

Datisca cannabina L.

Diapensiaceae

Galax aphylla L.

\section{Dipsacaceae}

Cephalaria alpina Schrad.

- tatarica Schrad.

Dipsacus Fullonum L.

- pilosus L. (32)

- silvester Huds.

Morina longifolia Huds.

Scabiosa atripurpurea Desf.

Droseraceae

Drosera capensis $\mathbf{L}$.

* - rotundifolia $\mathbf{L}$.

- spathulata Labill.

Elaeagnaceae

Elaeagaus multiflora Thunb.

Hippophae rhamnoides L.

Ericaceae

Andromeda floribunda Pursh

Calluna vulgaris Salisb.

Chamaedaphne calyculata Moench

Dabeocia cantabrica K. Koch 
Erica stricta Andr.

- vagans $L$.

Kalmia latifolia L.

Rhododendron maximum L.

- Smirnowii Trautv.

\section{Euphorbiaceae}

Acalypha brachystachya Hornem.

- virginica $L$.

Euphorbia bubalina Boiss.

- Cyparissias L.

- Gerardiana Jacq, (32)

- globosa Sims.

- helioscopia L.

- Lagascae Spreng.

- Lathyris $\mathbf{L}$.

- Myrsinitis L.

- platyphylla L.

- segetalis L.

Mercurialis annua $\mathbf{L}$.

Phyllanthus pulcher Wall.

Securinega fluegeoides Müll.-Arg.

\section{Fagaceae}

Quereus alba L.

\section{Gentianaceae}

Erythraea conferta Pers.

${ }^{*}$ Gentiana acaulis $\mathbf{L}$.

- asclepiadea L.

- cruciata L.

*- germanica Willd.

- lutea L.

- punctata L. (32)

- tibetica King

Hallenia elliptica D. Don

\section{Geraniaceae}

Erodium Botrys Bert.

- gruinum Ait.

- malacoides Willd.

- moschatum L'Hérit.
Geranium anemonifolium L'Hérit.

- dissectum L.

- incisum Nutt. (32)

- pratense $\mathbf{L}$.

- - L. var. albiflorum

- pyrenaicum L.

- rotundifolium $\mathbf{L}$.

- silvaticum L.

\section{Gesneraceae}

Alloplectus sanguineus Mart.

Besleria grandiflora $\mathrm{H}$. B. et K.

Klugia zeylanica Gardn.

Streptocarpus Rexii LdI. hybr.

\section{Globulariaceae}

Globularia Willkommii Nym.

\section{Guttiferae}

Hypericum hircinum L.

- hirsutum L.

- patulum Thunb.

- perforatum L.

- reptans Hook. f. et Thoms.

\section{Hamamelidaceae}

Fothergilla Gardenii Murr.

\section{Hydrophyllaceae}

Nemophila insignis Benth. (32)

- maculata Benth. (32)

Phacelia campanularia A. Gray (32)

- congesta Hook.

- grandiflora A. Gray

- malvaefolia Ch. et Schl.

- tanacetifolia Benth.

- Whitlavia A. Gray

Romanzoffia sitchensis Bong.

Juglandaceae

Juglans nigra $\mathbf{L}$.

Labiatae

Ajuga Chamaepitys Schreb. (32) 
Amethystea coerulea L.

Ballota nigra $\mathrm{L}$.

- rupestris Vis.

Brunella vulgaris $\mathrm{L}$.

- grandiflora Jacq.

Calamintha Clinopodium Spen.

- Nepeta Glairv.

Dracocephalum Moldavica L.

- rupestre Hance

- speciosum Benth.

Elsholtzia cristata Willd.

Galeopsis ochroleuca Lam.

- speciosa Mill.

- Tetrahit L.

Horminum pyrenaicum L.

Hyssopus officinalis $\mathbf{L}$.

Lallemantia iberica Fisch. et Mey.

- peltata Fisch. et Mey.

Lamium album L.

- Galeobdolon Crantz

- maculatum L.

Lavandula vera DC.

Leonurus Cardiaca L.

- sibiricus L.

Lycopus europaeus L.

Marrubium candidissimum L.

- vulgare L.

Mentha longifolia Huds.

Micromeria rupestris Benth.

Monarda fistulosa $\mathrm{L}$.

Nepeta Cataria L.

- grandiflora Bieb.

- japonica Maxim.

- nuda L.

- Veitchii Duth.

Ocimum Basilicum L.

- sanctum L.

Origanum vulgare $\mathbf{L}$.
Perilla ocymoides L.

- L. var. nankinensis

Phlomis spectabilis Falc.

- viscosa Poir.

Plectranthus fruticosus L'Hérit.

- glaucocalyx Maxim.

- Oertendahlii Th. Fries

Salvia austriaca Jacq.

- Bulleyana Diels

- cleistogama De Bary

- coccinea Juss.

- Jurisicii Kos.

- nemorosa L. (32)

- officinalis $\mathrm{L}$.

- patens Cav.

- pratensis L.

- Roemeriana Scheele

- Schiedeana Stapf

- silvestris L.

- verticillata $L$.

Satureja alpina Briq.

- hortensis $\mathbf{L}$.

Seutellaria albida L.

- alpina L.

- altissima L. (32)

- baicalensis Georgi

- dependens Maxim.

- galericulata L. (32)

Sideritis montana $\mathbf{L}$.

*Stachys Alopecuros Benth.

-- densiflorus Benth.

- germanica $\mathbf{L}$.

- lanata Jacq.

- officinalis Trev.

- silvatica L.

Teucrium Botrys L.

- hircanicum L.

* - Scorodonia L.

Thymus vulgaris $L$.

Leguminosae

Abrus precatorius L.

Amorpha fruticosa L. 
Astragalus baeticus L.

- Cicer L.

- danicus Retz.

- glyeyphyllos L.

Baptisia australis R. Br.

Cercis Siliquastrum $\mathbf{L}_{\text {. }}$

Cicer Arietinum L.

Colutea arborescens $\mathrm{L}$.

Coronilla scorpioides Koch

- varia $\mathbf{L}$.

Cytisus capitatus Jacq.

- nigricans $L$.

Desmodium canadense DC.

Dolichos ornatus Wall.

Dorycnium pentaphyllum Scop.

Galega officinalis L.

Genista sagittalis L. (32)

Glycine ussuriensis Rgl. et Maack

Glycyrrhiza echinata L.

- glabra L.

Halimodendron argenteum Fisch.

Hedysarum coronarium L.

- obscurum L.

Hippocrepis unisiliquosa $\mathbf{L}$.

Hosackia Purshiana Benth.

- Torreyi A. Gray

Laburnum vulgare Grisb.

Lathyrus alatus Ten.

- Gicera L.

- Clymenum L.

- hirsutus L.

- latifolius L.

- niger Bernh.

- Ochrus DG.

- sativus L.

- L. var. coeruleus

- silvester $\mathbf{L}$.

- vernus Bernh.

Leucaena glauca Benth.
Lotus corniculatus L.

- Tetragonolobus L.

Lourea vespertilionis Desf. (32)

Lupinus angustifolius $\mathbf{L}$.

- densiflorus Benth.

- luteus L.

- perennis $\mathrm{L}$.

Medicago hispida Gaertn.

- scutellata All.

- tribuloides Lam.

- tuberculata Willd.

Melilotus albus Med.

- altissimus Thuill.

- officinalis Lam.

Mimosa pudica L.

Onobrychis sativa Lam.

Ononis hircina Jacq.

- spinosa $\mathbf{L}$.

Ornithopus compressus L.

- sativus Brot

Phaseolus multiflorus Lam.

- vulgaris L. var. cerasiformis

- - L. var. nigerrimus

- - L. var. zebrinus

Psoralea Onobrychis Nutt.

Sarothamnus scoparius Wimm.

Scorpiurus suleata L.

Soja hispida Moench var. brunea

- - - - gracilis

- - - lutea

- - - nigra

Thermopsis fabacea DC.

Trifolium alexandrinum $\mathrm{L}$.

* - arvense $\mathbf{L}$.

- fragiferum L.

- incarnatum L.

- ochroleucum L.

- pratense $\mathbf{L}$.

- rubens $\mathbf{L}$.

Trigonella Foenum graecum L. 
Vicia atropurpurea Dest.

- dumetorum $\mathrm{L}$.

- Ervilia Willd.

- Faba L.

- L L. var. equina

- grandiflora Scop.

- lathyroides L.

- monanthos Desf.

- pubescens Link

- sativa L.

- vestita Boiss.

\section{Limnanthaceae}

Limnanthes alba Hartw.

- Douglasii R. Br.

\section{Linaceae}

Linum alpinum L.

- angustifolium Huds.

- austriacum L.

- capitatum Kit.

- flavum L.

- grandiflorum Desf. (32)

- hirsutum L. (32)

- maritimum L. (32)

- narbonense L.

- nervosum $W$. et $K$.

- perenne L.

- usitatissimum L.

\section{Loasaceae}

Blumenbachia Hieronymi Urb.

Cajophora lateritia Benth.

Loasa vulcanica Andr.

Mentzelia Lindleyi Torr. et Gray (32)

\section{Loganiaceae}

Spigelia splendens hort.

\section{Lythraceae}

Cuphea lanceolata Ait.

- viscosissima Jaeq.
Epilobium angustifolium L.

- Dodonaei Vill. (32)

- hirsutum L.

Lythrum Salicaria L.

Peplis portula L.

Malvaceae

Abutilon Avicennae Gaertn.

Althaea cannabina L.

- ficifolia Cav.

- officinalis L.

- rosea Cav.

- - var. nigra

Anoda Dilleniana Cav.

Callirrhoe pedata A. Gray

Hibiscus Trionum L.

- - L. var. africanus

Kitaibelia vitifolia Willd.

Lavatera trimestris L.

Malachra radiata L. (32)

Malva Alcea L.

- crispa L.

- silvestris L.

Pavonia praemorsa Gav.

- spinifex Gav.

Sida triloba Gav.

Martyniaceae

Martynia proboscidea Glox.

\section{Melastomataceae}

Calvoa orientalis Taub.

Dissotis plumosa Hook. f.

\section{Moraceae}

Cannabis sativa $\mathbf{L}$.

Dorstenia Contrajerva L.

Humulus Lupulus L.

Myricaceae

Myrica cerifera L. 
Myrsinaceae

Ardisia crispa A. DC.

Myrtaceae

Callistemon linearis DC.

Eugenia myrtifolia Sims.

Psidium Cattleyanum Sab.

Nolanaceae

Nolana atriplicifolia D. Don.

- prostrata L.

\section{Nyctaginaceae}

Mirabilis divaricata Lowe

- longifolia L.

Oxybaphus nyctagineus $\mathbf{L}$.

\section{Oenotheraceae}

Circaea cordata Royle

- lutetiana L.

Clarkia elegans Dougl.

Eucharidium grandiflorum F. et M.

Fuchsia boliviana Carr.

Gaura Drummondii Torr. et Gray.

Godetia amoena G. Don

- dasycarpa Phil.

- grandiflora Lindl.

- viminea Spach.

Lopezia coronata Andr.

Oenothera biennis $\mathrm{L}$.

- fruticosa L.

- Gigas Vries

- glauca Mchx.

- odorata Jacq.

- pumila L.

- rosea Ait.

- taraxacifolia Sw.

\section{Oleaceae}

Fraxinus Ornus L.

\section{Orobanchaceae}

Aeginetia indica L.

(auf Panicum plicatum Lam.)

* Orobanche flava Mart. (auf Petasites)

- lucorum A. Br. (auf Berberis)

- ramosa L. (auf Cannabis)

- speciosa DC. (auf Vicia Faba L.)

\section{Oxalidaceae}

Biophytum dormiens G. Don

Oxalis cernua Thunb. (bulb.)

- corniculata L. var. tropaeoloides

- Deppei Lodd. (bulb.)

- Iasiandra Zuce. (bulb.)

- pulchella Jacq. (bulb.)

- valdiviensis Barn.

- vespertilionis Zuce. (bulb.)

\section{Papaveraceae}

Adlumia cirrhosa Rafin.

Argemone albiflora Sms.

- mexicana L.

Boceonia cordata Willd.

Chelidonium majus $\mathbf{L}$.

Corydalis cheilanthifolia Hemsl.

- glauea Pursh

- lutea DC.

- ophiocarpa Hook. f. et Th.

- pallida Pers.

- sibirica Pers. (32)

- vaginans Royle

- Vesicaria Pers.

Dicentra eximia Torr.

Eschscholtzia caespitosa Benth.

- ealifornica Cham.

Fumaria officinalis Lam.

- parviflora Lam.

Glaucium corniculatum Curt.

- flavum Crantz

Hypecoum procumbens L.

Meconopsis cambriea Vig. 
Papaver alpinum L.

- dubium L.

- glaucum Boiss, et Hausskn.

- hybridum L.

- nudicaule L.

- orientale L.

- pavoninum Fisch. et Mey.

- rupifragum Boiss. et Reut.

- somniferum L.

- tauricolum Boiss.

Roemeria rhoeadiflora Boiss.

- violacea Medic.

Sanguinaria canadensis $L$.

Stylophorum Franchetianum Fedde

\section{Passifloraceae}

Passiflora gracilis Jacq.

\section{Phytolaceaceae}

Petiveria alliacea $\mathrm{L}$.

Phytolacea acinosa Roxb.

- decandra L.

- octandra L.

Rivina laevis L.

- tinctoria Ham.

\section{Piperaceae}

Peperomia blanda H. B. et K.

\section{Plantaginaceae}

Plantago arenaria W. et $\mathrm{K}$.

- aristata Mchx.

- Coronopus L.

- Cynops L.

- lanceolata L.

- major $\mathbf{L}$.

- maritima L.

- media L.

- patagonica Jacq.

- Psyllium L.

- Raoulii DC.
Plumbaginaceae

Armeria alpina Willd. (32)

- plantaginea Willd.

Statice globulariifolia Desf.

- Limonium L.

Polemoniaceae

Collomia coccinea Lehm.

- gilioides Benth.

- grandiflora Dougl.

- linearis Nutt.

Gilia achilleifolia Benth.

- capitata Sims.

- coronopifolia Pers.

- multicaulis Benth.

- squarrosa Hook. et Arn.

- tricolor Benth.

Polemonium coeruleum L.

- filicinum Greene

- pauciflorum S. Wats.

Polygonaceae

Emex spinosa Campd.

Muehlenbeckia axillaris Walp.

Oxyria digyna Hill.

Polygonum Bistorta L.

- capitatum Buch.-Ham.

- cuspidatum S. et $\mathrm{Z}$.

- Fagopyrum L.

- orientale L.

- Persicaria L.

- sachalinense F. Schmidt

- tinctorium Ait.

- Weyrichii Schmidt

Pterostegia drymarioides $\mathrm{F}$. et $\mathrm{M}$.

Rumex bucephalophorus L.

- crispus L.

- domesticus Hartm.

- flexuosus Sol.

- obtusifolius L.

- Patientia L.

- salicifolius Weinm.

- seutatus L. 


\section{Portulacaceae}

Anacampseros lanceolata Sweet

- rufescens DC.

Calandrinia compressa Schrad.

- discolor Schrad.

- grandiflora Lindl.

- Menziesii Hook.

- umbellata DC.

Portulacea grandiflora Hook.

- oleracea L.

- pusilla H. B. et K.

Talinum patens Willd.

Primulaceae

Anagallis arvensis L.

- L. var. coerulea

Androsace carnea L.

- elongata L.

- maxima L.

Asterolinon adoense Kze.

Cyclamen ibericum Goldie

Lysimachia punctata L.

- vulgaris L. var, dahurica

*Primula acaulis Jacq.

* Auricula L.

- Beesiana Forr.

- Bullesiana hort.

- Bulleyana Forr.

- burmanica I. B. Balf.

- cortusoides L.

- denticulata Sm.

- Sm. var. cashmiriana (32)

- elatior Jacq.

* f farinosa $L$.

- Florindae Ward.

- grandis Trautv.

- japonica A. Gray

- kewensis hort.

- longifolia All.

- mollis Hook.

- officinalis Jacq.
Primula Poissonii Franch.

- pulverulenta Duth.

- rosea Royle

- Veitchi Duth.

- vittata Bur. et Franch.

Samolus Valerandi L.

Ranunculaceae

Aconitum Anthora L. (32)

- Lycoctonum $\mathbf{L}$.

- Napellus L.

- paniculatum Lam.

- ranunculifolium Rchb.

- volubile Pall.

- Wilsonii Stapf

Actaea alba Mill.

- spicata L.

Adonis aestivalis L.

Anemone baldensis $\mathbf{L}$.

- dichotoma $\mathbf{L}$.

- japonica S. et Z. var. tomentosa

- multifida Poir.

- nemorosa L.

- Pulsatilla L.

- rivularis Buch.-Ham.

- silvestris L.

- virginiana $\mathrm{L}$.

Aquilegia alpina $\mathrm{L}$.

- vulgaris $\mathbf{L}$.

*_- -

Galtha palustris $\mathbf{L}$.

Cimicifuga racemosa Nutt.

Clematis heracleifolia DC.

- recta $L$.

- Vitalba L.

- Viticella L.

Delphinium Delavayi Franch.

- grandiflorum L. var. chinense

Eranthis eilicica Schott et Ky.

- hiemalis Salisb.

Helleborus foetidus L.

- purpurascens W. et K. 
Hydrastis canadensis L.

Leptopyrum fumarioides Rchb.

Myosurus minimus L.

Nigella damascena $L$.

- hispanica L.

- integrifolia Rgl.

- sativa L.

Paeonia corallina Retz.

- peregrina Mill. (32)

- Veitchii Lynch

Ranunculus acer L.

- aconitifolius L.

- arvensis $\mathrm{L}$.

- Cymbalaria Pursh

- Flammula L. (32)

- lanuginosus L.

- sceleratus L. (32)

Thalictrum angustifolium Jacq.

- aquilegifolium L.

- exaltatum Mey.

- minus $\mathbf{L}$.

Trollius europaeus L.

- Ledebourii Rchb.

- pumilus D. Don var. yunnanensis

\section{Resedaceae}

Rezeda alba L.

- crystallina Webb. et Bert.

- lutea L.

- Luteola L.

- odorata L.

- virgata Boiss. et Reut.

\section{Rosaceae}

Acaena Sanguisorbae Vahl.

Agrimonia Eupatoria L.

Alchemilla alpina $\mathbf{L}$.

- pubescens Link

- vulgaris $\mathbf{L}$.

Aronia arbutifolia Ell.

Aruncus silvester Kost.
Cotoneaster horizontalis Decne.

Crataegus Crus galli L.

*Dryas octopetala L.

Duchesnea indica Focke

Filipendula purpurea Maxim.

- Ulmaria Maxim.

var. elegans

Fragaria vesca $L$.

Geum macrophyllum Willd.

- rivale $\mathrm{L}$.

- strictum Ait.

- uniflorum I. Buch.

- urbanum L.

Gillenia trifoliata Moench

Holodiscus discolor Asch. et Graeb.

Malus floribunda Sieb.

Mespilus germanica L.

Photinia laevis Dipp.

Physocarpus opulifolius Raf.

Potentilla argentea L. var. calabra

- atrosanguinea Lodd.

- aurea L.

- canescens Bess.

- Hippiana Lehm.

- nepalensis Hook.

- norvegica $\mathrm{L}$.

- reeta $\mathrm{L}$.

- rupestris $\mathrm{L}$.

- sanguisorbifolia Wolf

- supina L.

- Tormentilla Neck.

Prunus pumila L.

- spinosa L.

Pyracantha coccinea Röm.

Rhodothypus kerrioides S. et Z.

Rosa canina L.

- dahurica Pall.

- gallica L.

- Moyesii Hemsl. et Wils.

- multiflora Thunb. 
Rosa rubiginosa L.

- setigera Rich.

- spinosissima L.

- virginiana Mill.

- Willmottiae Hemsl.

Rubus illecebrosus Focke

- odoratus L.

Sanguisorba minor Scop.

- officinalis L.

Sorbaria sorbifolia R. Br.

Sorbus domestica $\mathrm{L}$.

- torminalis Crantz

Stranvaesia Davidiana Dene.

\section{Rubiaceae}

Asperula arvensis L.

- eynanchica L.

- odorata L.

- orientalis Boiss. et Hoh.

- tinctoria L.

Galium Aparine L.

- Mollugo L.

- purpureum L.

- saccharatum AlI.

- silvaticum L.

Rubia tinetorum L.

Sherardia arvensis L.

\section{Rutaceae}

Dictamnus albus L.

Erythrochiton brasiliensis N. et M.

Ptelea trifoliata L.

Ruta bracteosa Dec.

- graveolens L.

\section{Sapindaceae}

Koelreutera paniculata Laxm.

\section{Saxifragaceae}

Astilbe Davidii Henry

Bergenía crassifolia Engl. (32)
Heuchera sanguinea Engl.

Peltiphyllum peltatum Engl.

Rodgersia podophylla A. Gray

- tabularis Kom.

Saxifraga Aizoon Jacq.

- altissima Kern.

- crustata Vest.

- Fortunei Hook.

- geranioides $\mathbf{L}$.

- granulata L.

- irrigua Bieb.

- Portae Stein

- rotundifolia $\mathrm{L}$.

Tellima grandiflora $\mathrm{R}$. Br.

Tiarella polyphylla D. Don

\section{Serophulariaceae}

Alonsoa caulialata $R$. et $P$.

- linearis R. et $P$.

Antirrhinum maurandioides A. Gray

- Orontium L.

Calceolaria chelidonioides H. B. et K.

- pinnata L. (32)

- scabiosaefolia Sims.

Chaenorrhinum minus Lange

_ _ - var. praetermissum

Chelone obliqua L.

Collinsia bicolor Benth.

Diascia Barberae Hook. f.

Digitalis ambigua Murr.

- ferruginea $\mathrm{L}$.

- lutea L.

- parviflora Jacq.

- purpurea L.

Erinus alpinus L.

Gratiola officinalis L.

Hebenstreitia dentata $\mathbf{L}$.

Limosella aquatiea L. 
Linaria alpina $\mathbf{L}$.

- chalepensis Mill.

- dalmatica Mill.

- genistifolia Mill.

- maroceana Hook.

- origanifolia DC.

- purpurea Mill.

- spartea Link et Hoffg.

- striata DC.

- supina Desf.

- Tournefortii Lge.

- triphylla Mill.

Maurandia Purpusii T. S. Brand

- semperflorens Ort.

Mimulus cardinalis Dougl.

- hybr. „Roter Kaiser"

- Inteus L.

- moschatus Dougl.

- pilosiusculus H. B. et K.

Nemesia floribunda Lehm.

- pubescens Benth.

- strumosa Benth.

- versicolor E. Mey.

Pentstemon campanulatus Willd,

- glaber Pursh

- secundiflorus Benth.

Phygelius capensis E. Mey.

Physostegia virginiana Benth.

Rhinanthus major Ehrh.

Serophularia alata Gilib.

- nodosa $\mathbf{L}$.

- Scopolii Hoppe

- vernalis $\mathbf{L}$.

Torenia peduncularis Benth.

Verbascum Blattaria L.

- longifolium Ten.

- nigrum L.

- olympicum Boiss.

- phoeniceum L.

- thapsiforme Schrad.
Veronica armena Boiss, et Huet.

- austriaca $\mathbf{L}$.

- Beccabunga L.

- Chamaedrys L.

- fruticulosa L.

- incana $\mathrm{L}$.

- longif olia L.

- officinalis $\mathrm{L}$.

- prostrata L.

- saxatilis Jaeq.

- spicata $\mathbf{L}$.

- spuria L.

\section{Solanaceae}

Atropa Belladonna L.

Browallia viscosa $H$. B. et $K$.

Capsicum annuum L.

Daturea arborea L. (32)

- inermis $\mathbf{L}$.

- Stramonium L.

- Tatula L.

Hyoseyamus niger $L$.

— L. var. pallidus

Lycopersicum esculentum Mill.

- Humboldtii Dun.

var, cerasiforme

Mandragora officinarum L.

Nicandra physaloides Gaertn.

Nicotiana affinis Moore

- Langsdorffii Weinm.

- longiflora Cav.

- nudicaulis S. Wats.

- rustica L.

- silvestris Speg. et Com.

- Tabacum L.

- L. var. macrophyllum

Nierembergia frutescens Dur.

Petunia nyctaginiflora Juss.

- violacea Lindl.

Physalis Alkekengi L.

- peruviana L. 
Salpiglossis sinuata Kuiz, et Pav.

Saracha edulis Thell.

Scopolia carniolica Jacq.

Solanum armatum R. Br.

- heterodoxum Dun.

- Dulcamara L.

- macrocarpum L.

- nigrum L.

- pyracanthum Jacq.

- villosum Link

Staphyleaceae

Staphylea colchica Scop.

- pinnata L.

\section{Sterculiaceae}

Hermannia alnifolia L.

Styracaceae

Halesia tetraptera L.

Thymelaeaceae

Daphne Mezereum L.

Thymelaea Passerina Cass, et Germ.

Tiliaceae

Tilia petiolaris DC.

- tomentosa Moench

\section{Tropaeolaceae}

Tropaeolum majus L.

- minus L.

\section{Ulmaceae}

Celtis australis L.

Umbelliferae

Aethusa Cynapium L.

Anethum graveolens L.

Apium tenuifolium Then.

Archangeliea officinalis Hoffm.

Astrantia major L.

Bupleurum falcatum L.

- filicaule Brot.

- rotundifolium L. (32)
Carum Carvi L.

Cenolophium Fischerei Koch Chaerophyllum aromaticum $\mathrm{L}$.

Cicuta virosa L.

Conium maculatum L.

Coriandrum sativum L.

Cryptotaenia canadensis DC.

Daucus Carota L.

- pulcherrimus Koch

Eryngium amethystinum L.

- giganteum Bieb.

- planum L.

Ferula communis L.

Foeniculum vulgare Mill.

Heracleum chorodanum DC.

- Lehmannianum Bge.

- Sphondylium L.

- villosum Fisch.

Levisticum officinale Koch

Meum athamanticum Jacq.

Myrrhis odorata Scop.

Oenanthe fistulosa L.

- Lachenalii Gmel.

- pimpinelloides $\mathbf{L}$.

Petroselinum sativum Hoffm.

Peucedanum officinale $\mathbf{L}$.

- Oreoselinum Moench

Pimpinella Anisum L.

- magna L.

- peregrina $\mathbf{L}$.

Sanicula-europaea L.

Scandix Pecten Veneris L.

Siler trilobum Scop.

Trachymene pilosa Sm.

Trinia vulgaris DC.

\section{Urticaceae}

Bowlesia tenera Spreng.

Laportea canadensis Gaud. (32)

- moroides Wedd. 
Parietaria officinalis $L$.

Pilea muscosa Lindl.

- Spruceana Wedd.

Urtica cannabina L.

- dioica $\mathrm{L}$.

- membranacea $\mathrm{L}$.

- urens L.

- pilulifera L.

Valerianaceae

Fedia Cornucopiae DC.

Valeriana officinalis $L$.

Valerianella Auricula DC.

- coronata DC.

- eriocarpa Desv.

\section{Verbenaceae}

Callicarpa Giraldiana Hesse

- japonica Thunb.

- longifolia Lam.

Lantana Camara L.
Verbena Aubletia L.

- bonariensis L. (32)

- officinalis $\mathbf{L}$.

- teucrioides Gill. et Hook.

- urticifolia L. (32)

- venosa Gill. et Hook. (32)

Violaceae

Hymenanthera crassifolia Hook.

Viola bosniaca Form.

- delphinifolia Nutt.

- Jooi Janka

- lutea Huds.

- mirabilis L.

- missouriensis Greene

- papilionacea Pursh

- Riviniana Rchb.

- Rydbergii Greene

- sagittata Ait.

- tricolor L. f. arvensis

Zygophyllaceae

Tribulus orientalis Kern.

- terrestris L.

Signum * indicat semina e plantis spontaneis lecta.

$(32)=$ semina anno 1932 lecta.

Für den Botanischen Garten der Universität:

Professor Dr. zur Strassen stellvertr. Direktor

$$
\text { Kiehne }
$$

Gartenoberinspektor
Für den städtischen

Zentral-Schulgarten:

Bromme

Städt. Gartenbaudirektor

$$
\text { Kiehne }
$$

Gartenoberinspektor 


\section{Frankfurt am Main}

Botanischer Garten der Universität und Zentral-Schulgarten der Stadt. INDEX SEMINUM

Desiderata inscribenda sunt: An die Direktion des Botanischen Gartens, Frankfurt-M.

\section{Pteridophyta}

Cyatheaceae

Dicksonia antaretiea Labill.

\section{Osmundaceae}

Osmunda regalis $\mathrm{L}$.

\section{Polypodiaceae}

Adiantum Capillus Veneris L.

- trapeziforme L.

Aspidium falcatum Sw.

- Filix mas Sw.

- spinulosum Sw.

Asplenium Belangeri Kze.

- bulbiferum Forst. var. Fabianum Bak.

- decussatum Sw.

- nidus $\mathrm{L}$.

- Petersonii Kze.

- Shepherdii Spr.

- Trichomanes L.

Athyrium Filix femina Roth

Blechnum brasiliense Desv.

- longifolium H. B. et K. var. fraxineum

- Moorei C. Chr.

Bak.

- Spicant Sm.

Davallia bullata Wall.

- Tyermanni Bak.

Doodia aspera R. Br.

Dryopteris patens. Kze. var. macroura

- Poiteana Urb.

KIfs.
Fadyenia prolifera Hook.

Gymnogramme calomelanos Klfs. var. Massonii Link.

- tartarea Desv.

Microlepia speluneae Moore

Nephrolepis biserrata Schott

Niphobolus Lingua I. Sm.

Platycerium alcicorne Desy.

Polypodium adnascens Sw.

- aureum L. var. cristatum hort.

- aureum L. var. glaucum hort.

- irioides Lam.

- Meyenianum Schott

- vacciniifolium $\mathrm{L}$. et $\mathrm{F}$.

- vulgare $L$.

Pteris biaurita L. var. argentea hort.

- cretica L. var. albolineata Hook.

- cretica L. var. Parkeri hort.

- longifolia L.

- tremula R. Br.

- umbrosa R. Br.

Scolopendrium Delavayi Franch.

- vulgare Sm.

Struthiopteris germanica Willd.

\section{Schizaeaceae}

Aneimia densa Link

- Dregeana Kze.

- Mandiocana Raddi

- Phyllitidis Sw.

- rotundifolia Schrad.

\section{Selaginellaceae \\ Selaginella Frimeliang}

- grandig Mogre: 6 ह

- Sieboldii Kze. 
Selaginella inaequalifolia Spring.

- Kraussiana A. Br.

\section{Gymnospermae}

Cupressaceae

Chamaecyparis Lawsoniana Parl.

\section{Pinaceae}

Larix leptolepis Gord.

Picea canadensis B. S. P.

Pinus excelsa Wall.

- Strobus L.

Pseudotsuga taxifolia Britt.

Taxaceae

Taxus baceata L.

- var. fastigiata Loud.

- var. adpressa Carr.

\section{Angiospermae}

\section{Monocotyledoneae}

Alismataceae

Alisma Plantago $\mathbf{L}$.

Amaryllidaceae

Ixiolirion Pallasii F. et M.

Vallota purpurea Herb.

Zephyranthes Atamasco Herb.

\section{Araceae}

Anthurium violaceum Schott

Arum maculatum L.

Calla palustris $\mathbf{L}$.

Xanthosoma Maximilianum Schott

\section{Bromeliaceae}

Acanthostachys strobilacea Link

Aechmea bromeliaefolia Bak.

Dyckia remotiflora Otto

- sulphurea C. Koch

\section{Commelinaceae}

Commelina coelestis Willd.

Palisota Barteri Hook f.

Tinantia fugax Scheidw.

\section{Cyperaceae}

Cyperus adenophorus Schrad.

- alternifolius L.

- elegans L.

- esculentus L. (tub.)

- flavescens L.

\section{Gramineae}

Aegilops crassa Boiss.

Agrostis nebulosa B, et R.

- stolonifera L.

Alopecurus pratensis $\mathbf{L}$.

Ammophila arenaria Link

Anthoxanthum odoratum L.

Apera Spica venti Bauv.

Aristida capillacea Lam.

Avena barbata Roth

- brevis Roth

- elatior L.

- flavescens L.

Boissiera bromoides Hochst. et Steud

Brachypodium distachyum Beauv.

- pinnatum Beauv.

- silvaticum R. et Sch.

Briza maxima $\mathrm{L}$.

Bromus asper Murr.

- erectus Huds.

- mollis L.

- secalinus L.

- squarrosus $\mathbf{L}$.

Calamagrostis epigeios Roth

Cenchrus echinatus L.

Chaeturus fasciculatus Link

Coix exaltata Jacq.

- Lacryma Jobis L.

Cynosurus cristatus L.

Dactylis glomerata $\mathrm{L}$.

Demaceria sicula Dum.

Deschampsia caespitosa Beauv.

- flexuosa Trin.

Eragrostis major Host.

- minor Host.

- nigra Nees

- pilosa Beauv.

Festuca dichotoma Forsk.

- duriuscula L.

- gigantea Vill.

- Halleri Auct. 
Festuca myurus L.

- pratensis Huds.

- pumila Vill.

- rubra L.

Heleochloa alopecuroides Host.

Holcus lanatus L.

Hordeum hexastichum L.

- jubatum L.

Ischaemum ciliare Retz.

Lagurus ovatus L.

Lamarkia aurea Moench

Lolium italicum A. Br.

- perenne L.

- remotum Schrank

Melica ciliata I.

Milium effusum L.

Molinia coerulea Moench

Muehlenbergia glomerata Trin.

Panieum bulbosum H. B. et K.

- capillare L.

- Crus galli L.

- miliaceum L.

Pennisetum typhoideum Rich.

Phalaris canariensis $\mathbf{L}$.

- minor Retz.

- paradoxa L.

Poa alpina L.

- nemoralis L.

- pratensis L.

- trivialis $\mathbf{L}$.

Polypogon monspeliensis Desf.

Setaria germanica Beauv.

- glauca Beauv.

- italica Beauv.

- verticillata Beauv.

Sorghum vulgare Pers.

- — f. nigrum

f. saccharatum

Spadiopogon sibiricus Trin.

Stipa sibirica Lam.

- tortilis Desf.

Tricholaena grandiflora Hochst.

Triticum dicoccum Schrank

- monococeum L.

Urachne trichotoma Trin.

Vilfa tenacissima H. B. et K.

Weingaertneria canescens Bernh.
Zea Mays L.

- - f. rubra

\section{Iridaceae}

Aristida Ecklonii Bak.

Crocus Fleischerei Gay

- speciosus M. Bieb.

Iris bucharica Foster

- dichotoma Pall.

- Hoogiana Dykes

- Kaempferi Sieb.

- notha M. Bieb.

- orientalis Thbg.

- pallida Lam.

- pumila L.

- sibirica L.

- warleyensis Foster

Lapeyrousea cruentha Benth.

Marica coerulea Ker.-Gawl.

- humilis Lodd.

Sisyrinchium anceps Cav.

- grandiflorum Dougl.

\section{Juncaceae}

Juncus lampocarpus Ehrh.

Luzula albida DC.

- nemorosa E. Mey.

- nivea DC.

\section{Liliaceae}

Agapanthus umbellatus L'Hérit.

— _ f. albiflorus

Allium angulosum L.

- azureum Ledeb.

- fistulosum L.

- flavum L.

- karataviense Regel

- Moly L.

- oleraceum L.

- Ostrowskianum Regel

- rotundum $\mathrm{L}$.

- Schoenoprasum L.

- Scorodoprasum L. (bulb.)

Anthericum Liliago L.

Asphodeline lutea Reichb.

Asphodelus Villarsii Verl.

Bowiea volubilis Harv.

Camassia esculenta Lindl.

Chlorophytum amaniense Engl.

- usambarense Engl. 
Cordyline stricta Endl.

Eremurus himalaicus Bak.

- Kaufmannii Regel

- robustus Regel

Fritillaria Meleagris L.

Galtonia candicans Dene.

Hosta coerulea Tratt.

- Sieboldiana Engl.

Kniphofia aloides Moench

Lilium Martagon L.

- regale E. H. Wils.

- superbum L.

Majanthemum bifolium Schmidt

Muscari racemosum Mill.

- botryoides Mill.

Ornithogalum narbonense L.

Polygonatum multiflorum All.

Puschkinia seilloides Adams

Scilla nonscripta Hoffm. et Link

- sibirica Andr.

Tulipa Sprengeri Bak.

Urginea maritima Bak.

Veratrum nigrum L.

Orchidaceae

Gymnadenia conopea R. Br.

Scheuchzeriaceae

Triglochin palustris $\mathrm{L}$.

Typhaceae

Typha latifolia L.

- minima Funk

Taceaceae

Tacea macrantha Jacq.

\section{Zingiberaceae}

Alpinia calcarata Rosc.

Globba heterobractea K. Schum.

- Schomburgkii Hook. f.

\section{Dicotyledoneae}

Acanthaceae

Aphelandra aurantiaca Lindl. var. Roezlii Regel
Ruellia Devosiana hort.

- strepens L.

Strobilanthes roseus Nees

\section{Aceraceae}

Acer cappadocicum Gleditsch

- circinatum Pursh.

- Ginnala Maxim.

- macrophyllum Pursh.

- tataricum L.

\section{Aizoaceae}

Mesembrianthemum eristallinum L.

- linguiforme $\mathrm{L}$.

- pyropaeum Haw.

\section{Amarantaceae}

Amarantus Blitum L.

- caudatus L.

- chlorostachys Wild.

- flavus L.

- hypochondriacus L.

- paniculatus L.

- viridis L. f. purpureus

Alternanthera sessilis $\mathrm{R}$. Br.

Gomphrena globosa L.

- Haageana Klotzsch

Telanthera polygonoides Moq.

Anacardiaceae

Rhus vernicifera DC.

Aquifoliaceae

Ilex glabra Gray

Aristolochiaceae

Aristolochia Sipho L'Hérit.

Asarum europaeum L.

Asclepiadaceae

Asclepias curassavica L.

Vincetoxicum officinale Moench

_ _ - f. purpurascens Beckh.

\section{Balsaminaceae}

Impatiens Balsamina L.

- biflora Walt.

- Holstii Engl. et Warb.

- Mathildae Chief.

- Noli tangere L. 
Impatiens parviflora DC.

- Roylei Walp.

Basellaceae

Basella rubra L.

Begoniaceae

Begonia Dregei Otto et Dietr.

\section{Berberidaceae}

Berberis Thunbergii DC.

- Wilsonae HemsI.

\section{Betulaceae}

Betula lenta L.

Bignoniaceae

Catalpa bignonioides Walt.

- ovata G. Don.

Eccremocarpus scaber $R$. et $P$.

Incarvillea Delavayi Bur. et Fr.

\section{Borraginaceae}

Anchusa officinalis L.

- sempervirens $\mathbf{L}$.

Caccinia strigosa Boiss.

Cerinthe major $\mathrm{L}$.

- minor L.

Cynoglossum amabile Stapf et Dr.

- coelestinum Lindl.

- officinale L.

Echinospermum Lappula Lehm.

Echium plantagineum L.

- vulgare $\mathrm{L}$.

Eritrichium strictum DC.

Heliotropium europaeum L.

Lithospermum arvense $L$.

- officinale $\mathrm{L}$.

- purpureo-coeruleum L.

Lycopsis arvensis $\mathbf{L}$.

Nonnea rosea Link

Omphalodes linifolia Moench

Cactaceae

Opuntia comanchica E. et B.

\section{Campanulaceae}

Campanula barbata L.
Gampanula carpatica Jacq.

- garganica Ten.

- lactiflora Bieb.

- latifolia L.

- patula L.

- pusilla Haenke

- rapunculoides $\mathrm{L}$.

- rotundifolia L.

- Scheuchzeri Vill.

- Trachelium L.

- turbinata Schott

Downingia pulchella Torr.

Hedraeanthus dalmaticus A. DC.

- dinaricus Wettst.

- tenuifolius DC.

Jasione montana L.

- perennis Lmk.

Lobelia Cliffortiana L.

- inflata L.

- syphilitica L.

Musschia Wollastoni Lowe

Phyteuma nigrum Schmidt

- spicatum L.

Platycodon grandiflorum A. DC.

Wahlenbergia gracilis Schrad.

- hederacea Reichb.

\section{Capparidaceae}

Cleome gigantea $\mathbf{L}$.

- graveolens Rafin.

\section{Caprifoliceae}

Sambucus racemosa L.

Symphoricarpus oceidentalis Hook.

Viburnum Opulus L.

— rhytidophyllum Hemsl.

Caryophyllaceae

Agrostemma Githago L.

Alsine saxatilis Crantz

Anychia dichotoma Michx.

Arenaria graminifolia Schrad.

- Ledebouriana Fenzl.

- serpyllifolia L.

Cerastium manticum L.

Dianthus Armeria L.

- banatieus Heuff.

- Carthusianorum L.

- gallicus Pers. 
Dianthus neglectus Loisel.

- pelviformis Heuff.

- plumarius L.

- Seguierii Vill.

- spiculifolius Schur.

Gypsophila altissima L.

- paniculata L.

- repens $\mathrm{L}$.

Lychnis alpina L.

- chalcedonica L.

- Coronaria Lmk.

- Flos cuculi L.

- - Jovis Desr.

- Viscaria L.

Melandryum album Garcke

- rubrum Garcke

Sagina procumbens L.

- subulata Torr. et Gray

Saponaria officinalis L.

Scleranthus annuus L.

Silene acaulis L.

- Armeria L.

- compacta Fisch.

- conica L.

- conoidea L.

- gallica L.

- italica Pers.

- linicola Gmel.

- noctiflora $L$.

- nutans L.

- quadrifida L.

- Schafta Gmel.

- vulgaris Garcke

Spergula arvensis $\mathrm{L}$.

Tunica prolifera Scop.

- Saxifraga Scop.

Vaccaria pyramidata Med.

\section{Celastraceae}

Evonymus alata Rgl.

- europaea L.

- latifolia Scop.

\section{Chenopodiaceae}

Atriplex nitens Schkuhr

- patulum L.

- sibiricum L.

Chenopodium album L.

- ambrosioides L.

- Botrys L.
Chenopodium hybridum L.

- murale L.

- opulifolium Schrad.

- polyspermum L.

- rubrum L.

- urbicum L.

- Vulvaria L.

Corispermum hyssopifolium L.

Hablitzia tamnoides Bieb.

Kochia scoparia Schrad.

Salsola Kali L.

- Soda L.

\section{Cistaceae}

Helianthemum guttatum Mill.

- vulgare Gaertn.

\section{Clethraceae}

Clethra acuminata Michx.

- alnifolia L.

\section{Compositae}

Achillea Clavennae L.

- Millefolium L.

Anacyclus officinarum Hayn.

Antennaria imbricata E. Nels.

Anthemis tinctoria L.

Arctium Lappa L.

Aretotis grandis Thunb.

Arnica longifolia Eaton

- montana L.

Artemisia Absinthium L.

- vulgaris $\mathbf{L}$.

Aster himalaicus C. B. Clarke

- incisus Fisch.

- Linosyris Bernh.

- subcoeruleus L. Moore

- Tripolium L.

Bellium minutum $\mathbf{L}$.

Bidens leucanthus Willd.

- tripartitus L.

Brickellia grandiflora Nutt.

Buphthalmum speciosum Schreb.

Carduus acanthoides L.

- nutans L.

- stenolepis Benth.

Carlina caulescens Lmk.

- vulgaris L. 
Carthamus tinctorius $\mathrm{L}$.

Catananche lutea L.

Centaurea Crocodylium L.

- cynaroides Link

- Jacea L.

- Lippii L.

- montana L.

- nigra L.

- pulcherrima Willd.

- Sadleriana Janka

- Scabiosa L.

Chrysanthemum caucasicum Pers.

-. corymbosum L.

- Leucanthemum L.

- macrotum Ball.

- viscosum Desf.

- vulgare Bernh.

Cirsium Diacantha DC.

- eriophorum Scop.

- oleraceum Scop.

- pulchellum W. et St.

- Velenowskii Vaud.

Cnicus benedictus L.

- syriacus Roth

Coreopsis Atkinsoniana Dougl.

- coronata Hook.

- grandiflora Nutt.

Cotula coronopifolia L.

- turbinata L.

Crepis blattarioides Vill.

- Dioscoridis L.

- rubra L.

- sibirica L.

- tectorum L.

Cynara Scolymus L.

Dahlia Merckii Lehm.

Doronicum caueasicum Bieb.

- Pardalianches L.

Erigeron speciosus DG.

Eupatorium cannabinum L.

- ageratoides $\mathrm{L}$.

- purpureum L.

Felicia tenella Nees

Gnaphalium purpureum L.

Grindelia squarrosa Pursh.

Hedypnois cretica Willd.

Helenium Hoopesi A. Gray

Helianthus annuus $\mathbf{L}$.

- debilis Nutt.
Helichrysum arenarium DC.

- foetidum Moench

- orientale Gaertn.

Helipterum corymbiflorum Schldl.

- Humboldtianum DC.

- Manglesii F. Muell.

Hieracium aurantiacum L.

- Bornmuelleri Freyn.

- bupleuroides Gmel.

- canadense Michx.

- chlorocephalum Wim.

- laevigatum Willd.

- murorum L.

- pannosum Boiss.

- silvetre Tausch

_ villosum L.

- vulgatum Fries

Hypochoeris glabra L.

- radicata $\mathbf{L}$.

Inula glandulosa Puschk.

- Helenium L.

Koelpinia linearis Pall.

Lactuca muralis Less.

- Scariola L.

- virosa L.

Lasthenia glabrata Lindl.

Leontopodium alpinum Cass.

Leptosyne maritima A. Gray

Liatris elegans Willd.

— spicata Willd.

Ligularia sibirica Cass.

Moscharia pinnatifida R. et P.

Onopordon Aeanthium L.

Picridium tingitanum Desv.

Picris echioides L.

Relhania sessilifiora Thunb.

Rhagadiolus stellatus Willd.

Rodigia commutata Spreng.

Rudbeckia amplexicaulis Vahl.

- purpurea L.

- speciosa Wend.

Seorzonera parviflora Jacq.

Senecio clivorum Maxim.

- Fuchsii Gmel.

- macrophyllus Bieb.

- Veitchianus Hemsl.

- Wilsonianus Hemsl.

Siegesbeckia orientalis $\mathbf{L}$. 
Silphium perfoliatum L.

Silybum Marianum Gaertn.

Solidago Virga aurea L.

Sonchus palustris $\mathbf{L}$.

Taraxacum officinale Web.

Tolpis barbata Gaertn.

Tragopogon major Jacq.

- porrifolius $\mathrm{L}$.

Tridax trilobata Hemsl.

Troximon gracilens A. Gray

Ursinia anthemoides Gaertn.

Verbesina encelioides Benth. et Hook.

Xanthium spinosum $L$.

Xeranthemum inapertum Mill.

- longepapposum Fisch. et Mey.

Zinnia pauciflora L.

- verticillata Andr.

\section{Convolvulaceae}

Convolvulus dahuricus Sims.

- elongatus Willd.

- undulatus Cav.

Cuscuta Epilinum Weihe

Ipomoea Bona nox L.

- purpurea Roth

\section{Cornaceae}

Cornus Baileyi Coult. et Ev.

- mas L.

- pubescens Nutt.

\section{Crassulaceae}

Bryophyllum crenatum Bak.

Kalanchoë glaucescens Britten

- rotundifolia Haw.

Sedum maximum Sut.

- reflexum L.

Sempervivum annuum C. $\mathrm{Sm}$.

\section{Cruciferae}

Aethionema saxatile R. Br.

Alliaria officinalis Andr.

Alyssum calycinum L.

- maritimum Lam.

- Moellendorfianum Aschers.

- petraeum Ard.

- saxatile $\mathrm{I}$.
Arabis alpina L.

- arenosa Scop.

- perfoliata Lam.

- procurrens W. et $\mathbf{K}$.

Barbaraea vulgaris $\mathrm{R}$. Br.

Biscutella apula L.

- auriculata L.

- ciliata DC.

- didyma L.

Brassica Napus L.

- nigra Koch

- oleracea L.

- Rapa L.

Camelina sativa Crantz

Capsella grandiflora Boiss.

- Viguierii Blar.

Cardamine chenopodiifolia Pers.

- graeca L.

- hirsuta L.

- pratensis $\mathrm{L}$.

Coronopus procumbens Gilib.

Crambe cordifolia Stev.

- maritima $\mathbf{L}$.

Diplotaxis tenuifolia DG.

Draba Aizoon Wahlenb.

- repens Bieb.

Eruca sativa Lmk.

Erysimum cheiranthoides L.

- Perowskianum F. et M.

Hesperis matronalis $\mathbf{L}$.

Iberis Lagascana DC.

- taurica DC.

Isatis Boisseriana Reichb.

— tinctoria L.

Lepidium sativum L.

Lunaria biennis Moench

- rediviva $\mathbf{L}$.

Malcomia maritima Ait.

Matthiola bicornis DC.

- tricuspidata R. Br.

Nasturtium officinale R. Br.

Neslea paniculata Desv.

Ricotia Lunaria.DC.

Sinapis Alba L.

Sisymbrium strictissimum $\mathrm{L}$.

Vesicaria utriculata Lmk. 


\section{Cucurbitaceae}

Bryonia alba $\mathrm{L}$.

- dioica Jacq.

Cyclanthera explodens Naud.

- pedata Schrad.

Ecballium Elaterium A. Rich.

\section{Datiseaceae}

Datisea cannabina L.

\section{Dipsacaceae}

Cephalaria tatarica Schrad.

Dipsacus Fullonum L.

- pilosus $\mathbf{L}$.

- silveter Huds.

Morina longifolia Wall.

Seabiosa maritima L.

\section{Elaeagnaceae}

Elaeagnus longipes A. Gray

\section{Ericaceae}

Dabeocia polifolia D. Don.

Vaccinium Vitis idaea $\mathbf{L}$.

\section{Euphorbiaceae}

Euphorbia bubalina Boiss,

- Cyparissias L.

- exigua L.

- Gerardiana Jacq.

- Lagascae Spreng.

- Lathyris L.

Mercurialis annua $\mathrm{L}$.

Ricinus communis $\mathrm{L}$.

\section{Fagaceae}

Quercus alba L.

\section{Gentianaceae}

Gentiana asclepiadea L.

- cruciata L.

- punctata L.

- septemfida Pall.

- tibetica King

\section{Geraniaceae}

Erodium cicutarium L'Hérit.

- malacoides Willd.

Geranium carolinianum L.

- incisum Nutt.
Geranium pratense L.

— - f f. albiflorum

- pyrenaicum L.

- Robertianum L.

- rotundifolium L.

- silvaticum L.

Pelargonium alchemilloides L'Hérit.

- malvaefolium Jacq.

\section{Gesneraceae}

Alloplectus capitatus Hook.

Klugia zeylanica Gardn.

\section{Guttiferae}

Hypericum patulum Thunb.

- perforatum $\mathbf{L}$.

- polyphyllum Boiss. et Bal.

- pulchrum L.

- Webbii Steud.

\section{Hamamelidaceae}

Fothergilla Gardenii Murr.

\section{Hydrophyllaceae}

Ellisia Nyctelaea L.

Nemorphila insignis Benth.

Phacelia campanulata A. Gray

- congesta Hook.

- grandiflora A. Gray

- tanacetifolia Benth.

- viscida Torr.

\section{Labiatae}

Ballota nigra $L$.

Brunella vulgaris $\mathbf{L}$.

Calamintha officinalis Moench

Clinopodium vulgare L.

Elsholtzia cristata Willd.

Galeopsis speciosa Mill.

- Tetrahit L.

Horminum pyrenaicum L.

Hyssopus officinalis L.

Lamium album L.

- Galeobdolon Crantz

- Orvala L.

Lavandula officinalis Chaix

Leonurus Cardiaca L.

- sibiricus L.

Melittis Melissophyllum L. 
Monarda citriodora Cerv.

Nepeta Cataria L.

- grandiflora Bieb.

- nuda L.

Ocimum Basilieum L.

- sanctum L.

Origanum vulgare $\mathrm{L}$.

Perilla ocymoides L.

Plectranthus glaucocalyx Maxim.

Salvia cleistogama De Bary

- coccinea Juss.

- Jurisicii Kos.

- nemorosa L.

- officinalis L.

- pratensis L.

- Schiedeana Stapf.

- Sclarea L.

- silvestris L.

- verticillata L.

Satureja alpina Briq.

- hortensis L.

Scutellaria albida L.

- altissima L.

- baicalensis Georgi

- galericulata L.

Stachys densiflora Benth.

- germanica L.

- iberica Bieb.

- officinalis Trev.

- silvatica L.

Teucrium Botrys L.

- Scorodonia L.

Thymus vulgaris $L$.

\section{Leguminosae}

Astragalus baeticus L.

- Cicer L.

- filicaulis Fisch. et Mey.

- glycyphyllos L.

Baptisia australis $\mathrm{R}$. Br.

Colutea arborescens $\mathrm{L}$.

Coronilla scorpioides Koch

- varia L.

Cytisus capitatus Jacq.

Dolichos ornatus Wall.

Galega officinalis L.

Genista sagittalis L.

Glyeyrrhiza glabra L.
Halimodendron argenteum Fisch.

Laburnum alpinum Grisb.

- vulgare Grisb.

Lathyrus hirsutus L.

- latifolius L.

- niger Bernh.

- Nissolia L.

- sativus L.

- nernus Bernh.

Lotus Tetragonolobus L.

Lourea vespertilionis Desf.

Lupinus albus L.

- angustifolius L.

- arboreus Sims.

-- luteus L.

- mutabilis Sweet

- perennis $\mathbf{L}$.

- sericeus Pursh.

Medicago hispida Gaertn.

- lupulina L.

- orbicularis All.

- radiata $L$.

- sativa L.

- scutellata All.

Melilotus albus Desr.

- altissimus Thuill.

- officinalis Lam.

Mimosa pudica L.

Onobrychis sativa Lmk.

Ononis hircina Jaeq.

- spinosa L.

Ornithopus sativus Brot.

Phaseolus capensis Thunb.

- multiflorus Lmk. var. coccineus L.

- vulgaris f. atropunctatus

- - f. zebrinus

Sarothamuus scoparius Wimm.

Soja hispida Moench

Tetragonolobus purpureus Moench

Trifolium incarnatum $\mathbf{L}$.

- pannonicum L.

- pratense L.

- rubens $\mathrm{L}$.

Trigonella coerulea Ser.

- Foenum graecum L.

Ulex europaeus L.

Vicia dumetorum L.

- Faba L. 
Vicia pubescens Link.

- sicula Guss.

- vestita Boiss.

\section{Limnanthaceae}

Limnanthes Douglasii R. Br.

\section{Linaceae}

Linum angustifolium Huds.

- austriacum L.

- capitatum Kit.

- flavum L.

- grandiflorum Desv.

- hirsutum L.

- maritimum L.

- nervosum W. et K.

- usitatissimum L.

\section{Loasaceae}

Blumenbachia Hieronymi Urb.

Cajophora lateritia Benth.

Loasa tricolor Lindl.

\section{Loganiaceae}

Spigelia splendens hort.

Lythraceae

Cuphea lanceolata Ait.

- viscosissima Jacq.

Epilobium angustifolium L.

- Dodonaei Vill.

- Fleischerei Hochst.

- hirsutum L.

- hypericifolium Tausch.

- obscurum Schreb.

Godetia amoena G. Don.

Lythrum Graefferi Ten.

- Salicaria L.

\section{Malvaceae}

Althaea canuabina L.

- ficifolia Cav.

- officinalis L.

- rosea Cav.

Anoda cristata Schlecht.

Hibiscus syriacus $\mathbf{L}$.

Malachra radiata L.

Malva Alcea L.

- Meluca Graebn.

- neglecta Wallr.
Pavonia praemorsa Cav.

- spinifex Cav.

Sidalcea malvaeflora A. Gray

Melastomataceae

Bertolonia marmorata Naud.

- pubescens hort.

Galvoa orientalis Tomb.

- sessiliflora Tomb.

Dissotis plumosa Hook. f.

Moraceae

Dorstenia Contrajerva L.

Humulus Lupulus L.

Myrtaceae

Callistemon coccineus F. Muell.

- linearis DC.

Myrtus communis L.

Psidium Cattleianum Sabine

Nolanaceae

Nolana prostrata L.

Nyctaginaceae

Mirabilis divaricata Lowe

- longiflora L.

Oxybaphus nyctagineus Sweet

\section{Oenotheraceae}

Circaea cordata Royle

- lutetiana L.

Eucharidium grandiflorum F. et M.

Oenothera biennis L.

- missouriensis Sims.

- pumila L.

- taraxacifolia Sw.

\section{Oleaceae}

Fraxinus Ornus L.

Ligustrum japonicum Thunb.

- vulgare L.

\section{Orobanchaceae}

Aeginetia indica $\mathrm{L}$.

Orobanche lucorum A. Br.

- ramosa L.

- speciosa DC. 
Oxalidaceae

Biophytum dormiens G. Don.

Oxalis Deppei Lodd. (bulb.)

\section{Papaveraceae}

Adlumia fungosa Greene

Argemone mexicana $L$.

- platyceras Link et Otto

Bocconia cordata Willd.

Chelidonium majus $\mathbf{L}$.

Corydalis cheilanthifolia Hemsl.

- glauca Pursh.

- Intea DC.

- ophiocarpa Hook. f. et Thoms.

- sibirica Pers.

- Vesicaria Pers.

Eschscholtzia californica Cham.

- tenuifolia Benth.

Claucium flavum Crantz

Meconopsis cambrica Vig.

Papaver alpinum $\mathrm{L}$.

- californicum A. Gray

- glaucum Boiss. et Hausskn.

- nudicaule L.

- orientale L.

- pavoninum C. A. Mey.

- rupifragum Boiss. et Reut.

- somniferum L.

Stylophorum Franchetianum Fedde

\section{Phytolaceaceae}

Petiveria alliacea $\mathrm{L}$.

Phytolacca decandra L.

- heteropetala H. Walt.

Rivina laevis $\mathbf{L}$.

- tinetoria Ham.

\section{Plantaginaceae}

Plantago alpina $\mathrm{L}$.

- amplexicaulis Cav.

- arborescens Poir.

- arenaria W. et $\mathbf{K}$.

- asiatica L.

- Coronopus L.

- lanceolata L.

- major L.

- maritima L.

- maxima Jacq.

- media L.

- virginica L.
Plumbaginaceae

Armeria alpina Willd.

- maritima Willd.

Polemoniaceae

Collomia coccinea Lehm.

- grandiflora Dougl.

- linearis Nutt.

Gilia achilleaefolia Benth.

- californica Benth.

- capitata Sims.

- tricolor Benth.

Polemonium coeruleum L.

- filicinum Greene

- pauciflorum S. Wats.

\section{Polygonaceae}

Emex spinosa Campd.

Oxyria digyna Hill.

Polygonum Bistorta L.

- cuspidatum $S$. et $Z$.

- Fagopyrum L.

- orientale L.

- Persicaria L.

- tinctorium Ait.

Rheum palmatum $\mathbf{L}$.

Rumex acetosa L.

- bucephalophorus $\mathrm{L}$.

- maritimus L.

- Patientia L.

- salicifolius Weinm.

- scutatus L.

\section{Portulacaceae}

Anacampseros rufescens DC.

Calandrinia compressa Schrad. discolor Schrad.

- Menziesii Torr.

Claytonia perfoliata Donn.

- sibirica L.

Portulaca oleracea L.

\section{Primulaceae}

Anagallis arvensis L.

- - f. coerulea

Androsace septentrionalis L.

Asterolinum stellatum Hoffm.

Dodecatheon Meadia L.

Primula acaulis Jacq.

- arctotis $\times$ Kern. 
Primula Beesiana Forrest

- denticulata Sm.

- - var. cashmeriana Hook. f.

- elatior Jacq.

- farinosa L.

- Florindae Ward.

- grandis Trautv.

- involucrata Wall.

- officinalis Jaeq.

- pulverulenta Duth.

- saxatilis Kom.

- spectabilis Tratt.

- vittata Bur. et Franch.

Samolus Valerandi L.

\section{Ranunculaceae}

Aconitum Anthora L.

- Lycoctonum L.

- Napellus L.

- Stoerkianum Reichb.

- tauricum Wulf.

- volubile Pall.

Actaea alba Mill.

- spicata L.

Anemone apennina L.

- baldensis L.

- dichotoma L.

- japonica S. et $Z$. var. hupehensis

- nemorosa L. hort.

- Pulsatilla L.

- rivularis Buch.-Ham.

- virginiana $\mathrm{L}$.

Aquilegia alpina L.

- vulgaris L.

Caltha palustris $\mathbf{L}$.

Cimicifuga cordifolia Pursh.

Clematis recta $\mathrm{L}$.

- Viticella L. f. rubra

Delphinium caucasicum C. A. Mey.

- Delavayi Franch.

- formosum Boiss.

- hybridum Willd.

- sinense Fisch.

- Staphysagria L.

- turkestanicum Huth.

Eranthis hiemalis Salisb.

Helleborus foetidus L.

- purpurascens W. et K.

Nigella damascena $\mathrm{L}$.

- sativa L.
Paeonia corallina Retz.

- officinalis $\mathbf{L}$.

- peregrina Mill.

- Veitchii Lynch

Ranunculus abortivus $\mathrm{L}$.

- acer L.

- arvensis L.

- Flammula L.

- ophioglossifolius Vill.

- polyanthemos L.

- sceleratus L.

Thalictrum aquilegifolium $\mathrm{L}$.

- glaucum Desf.

- minus L. var. flexuosum Bernh.

Trollius asiaticus $\mathrm{L}$.

- chinensis Bunge

- europaeus L.

- Ledebourii Reichb.

\section{Resedaceae}

Reseda alba L.

- crystallina Webb. et Berth.

- inodora Reichb.

- lutea L.

- Luteola L.

- mediterranea L.

- ramosissima Pourr.

\section{Rhamnaceae}

Ceanothus americanus L.

Rhamnus cathartica L.

\section{Rosaceae}

Agrimonia Eupatoria L.

- odorata Mill.

Alchemilla alpina L.

- pubescens Lmk.

Aruncus silvester Kostel

Gotoneaster horizontalis Decne.

Dryas octopetala L.

Filipendula camtschatica Maxim.

- Ulmaria Maxim.

Fragaria indica Andr.

Geum rivale L.

- urbanum L.

Holodiscus discolor Asch. et Graebn.

Mespilus germanica L.

Physocarpus opulifolius Raf.

Pimpinella magna L.

- peregrina $\mathbf{L}$. 
Potentilla alba L.

- argentea L. var. calabra Ser.

- atrosanguinea Lodd.

- canescens Bess.

- collina Wibel

- nepalensis Hook.

- norvegica L.

- recta $L$.

- rupestris $\mathrm{L}$.

- Salesowiana Steph.

- supina L.

Pyracantha coccinea Röm.

Rhodotypus kerrioides S. et Z.

Rosa eanina L.

- Moyesii Hemsl. et Wils.

- pisicarpa A. Gray

- spinosissima $\mathbf{L}$.

- setigera Rich.

- virginiana Mill.

Sanguisorba canadensis L.

- minor Scop.

- officinalis L.

Sorbaria sorbifolia A. Br.

\section{Rubiaceae}

Asperula arvensis $\mathrm{L}$.

- odorata L.

- orientalis Boiss. et Hoh.

Galium Aparine L.

- Mollugo L.

- tricorne Stokes

- verum L.

Sherardia arvensis L.

\section{Rutaceae}

Dictamnus albus $\mathrm{L}$.

Erythrochiton brasiliensis N. et M.

Ptelea trifoliata L.

Ruta graveolens L.

\section{Salicaceae}

Salix retusa $\mathrm{L}$.

\section{Sapindaceae}

Koelreuteria paniculata Laxm.

\section{Saxifragaceae}

Astilbe Davidii Henry

Bergenia erassifolia Engl.

Heuchera americana $L$.
Rodgersia aesculifolia A. Gray

- sambucifolia Hemsl.

- podophylla A. Gray

Saxifraga Aizoon Jacq.

- granulata L.

- peltata Torr.

- Portae Stein.

Tiarella cordifolia L.

Serophulariaceae

Antirrhinum glutinosum Boiss. et Reut.

- Orontium L.

Calceolaria pinnata $\mathrm{L}$.

- seabiosaefolia Sims.

Chelone obliqua $\mathrm{L}$.

Diascia Barberae Hook. f.

Digitalis ambigua Murr.

- ferruginea L.

- lutea L.

- purpurea L.

Erinus alpinus L.

Gratiola officinalis L.

Linaria alpina $\mathrm{L}$.

- bipartita Willd.

- canadensis Dum.-Cours.

- chalepensis Mill.

- dalmatica Mill.

- macedonica Griseb.

- maroccana Hook.

- striata DC.

- triphylla Mill.

Maurandia Purpusii T. S. Brand.

Mimulus cupreus Rgl.

- luteus L.

Scrophularia alata Gilib.

- altaica Murr.

- nodosa L.

- sambucifolia L.

- Scopolii Hoppe

- vernalis L.

Verbascum Blattaria L.

- nigrum $\mathbf{L}$.

- thapsiforme Schrad.

Veronica austriaca L.

- Chamaedrys L.

- fruticulosa L.

- officinalis L.

- saxatilis Jacq.

- spicata L. 
Solanaceae

Atropa Belladonna L.

Datura Bernhardii Lundstr.

- Stramonium L.

- Tatula L.

Hyoseyamus niger $L$.

- - var. pallidus W. et R.

Lycopersicum esculentum Mill. var. cerasiforme $\mathbf{L}$.

- Humboldtii Dun.

- pimpinellifolium Mill.

Nicandra physaloides Gaertn.

Nicotiana acutiflora A. St. Hil.

- affinis Moore

- fragrans Hook.

- fruticosa L.

- glutinosa $\mathbf{L}$.

- nudicaulis S. Wats.

- plumbaginifolia Viv.

- quadrivalvis Pursh.

- rustica L.

- silvestris Speg. et Comes

- Tabacum L.

- viscosa Lehm.

Petunia parviflora Juss.

Physalis Alkekengi L.

Schizanthus pinnatus $R$. et $P$.

Solanum citrullifolium A. Br.

- Dulcamara L.

- macrocarpum L.

- nigrum L.

- pyracanthum Jacq.

- villosum Lmk.

Staphyleaceae

Staphylea colchica Scop.

Styracaceae

Halesia tetraptera L.

Thymelaeaceae

Daphne Mezereum L.

\section{Tiliaceae}

Tilia petiolaris DC.

- tomentosa Moench

\section{Tropaeolaceae}

Tropaeolum aduncum Sm.

- majus L.
Tropaeolum majus L. var. nanum hort.

- minus L.

Ulmaceae

Celtis australis $\mathrm{L}$.

\section{Umbelliferae}

Aethusa Cynapium L.

Angelica pyrenaea $S_{p r}$.

Anthriscus silvestris Hofim.

Apium tenuifolium Then.

Archangelica officinalis Hoffm.

Astrantia major L.

Bupleurum faleatum L.

- rotundifolium $\mathrm{L}$.

Carum Bulbocastanum Koch

- Carvi L.

Caucalis daucoides L.

Chaerophyllum temulum L.

Cicuta virosa $\mathbf{L}$.

Conium maculatum $\mathrm{L}$.

Coriandrum sativum L.

Cryptotaenia canadensis DC.

Elaeoselinum foetidum Boiss.

Eryngium alpinum L.

- planum L.

Falcaria vulgaris Bernh.

Foeniculum officinale All.

Heracleum Sphondylium L.

— villosum Fisch.

Levisticum officinale Koch

Meum Athamanticum Jacq.

Myrrhis odorata Scop.

Oenanthe fistulosa $L$.

- pimpinelloides $\mathbf{L}$.

Pastinaca sativa L.

Petroselinum sativum Hoffm.

Peucedanum officinale L.

- Oreoselinum Moench

- Ostruthium Koch

Sanicula europaea L.

Scandix Pecten Veneris L.

Seseli Libanotis Koch

Siler trilobum Scop.

Sium angustifolium $\mathrm{L}$. 
Sium latifolium L.

- Sisarum L.

Thaspium aureum Nutt.

\section{Urticaceae}

Laportea canadensis Gaud.

- moroides Wedd.

Parietaria officinalis L.

- ramiflora Moench

Pilea muscosa Lindl.

- Spruceana Wedd.

Urera baccifera $L$.

Urtica cannabina L.

$\sim$ pilulifera $\mathrm{L}$.

\section{Valerianaceae}

Valeriana officinalis L.

\section{Verbenaceae}

Callicarpa japonica Thunb.

Lantana Sellowiana Link et Otto

Für den Botanischen Garten der Universität:

Professor Dr. zur Strassen

stellvertr. Direktor

Kiehne

Garteninspektor
Verbena bonariensis $\mathrm{L}$.

- officinalis $\mathrm{L}$.

- urticifolia L.

- venosa Gill. et Hook.

\section{Violaceae}

Viola alichariensis G. Bech.

- calaminaria Lej.

a cucullata Ait.

- delphinifolia Nutt.

- elegantula Schott

- hederacea Labill.

- Jooi Janka

- macroceras Bge.

- missouriensis Greene

- papilionacea Pursh.

- odorata L.

- Patrinii Ging.

- persicifolia Roth

- Riviniana Reichb.

- rothomagensis Desf.

- Rydbergii Greene

- sagittąta Ait.

- splendida W. Beck.
Für den städtischen Zentral-Schulgarten:

Bromme

Städt. Gartenbaudirektor

Kiehne

Garteninspektor 


\section{SAMEREIEN}

\section{zum Tausch angeboten}

vom

Bołanischen Garten der Universität

Frankfurt am Main

J a n a r 1934

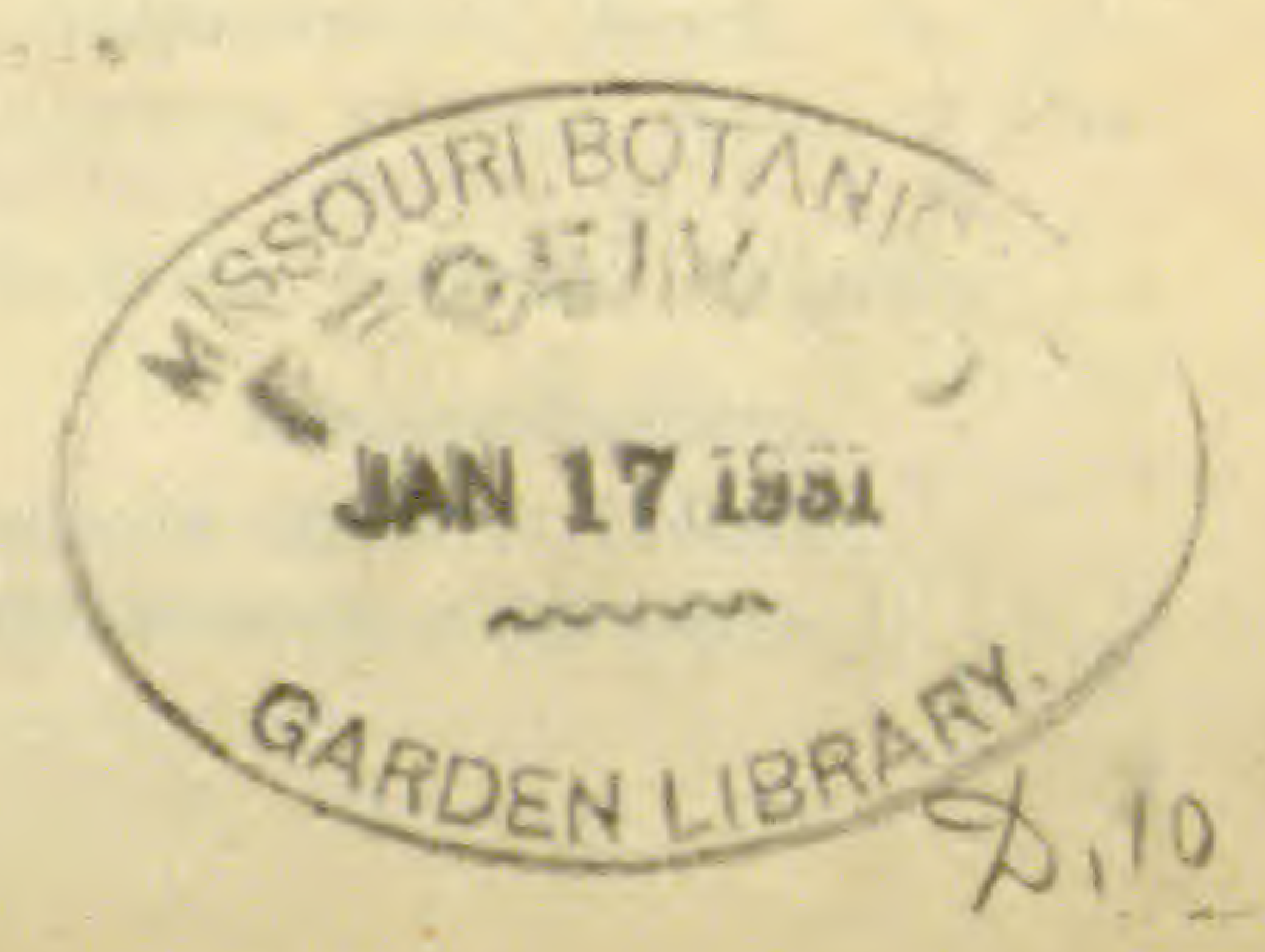




\section{MEIFSTMMR

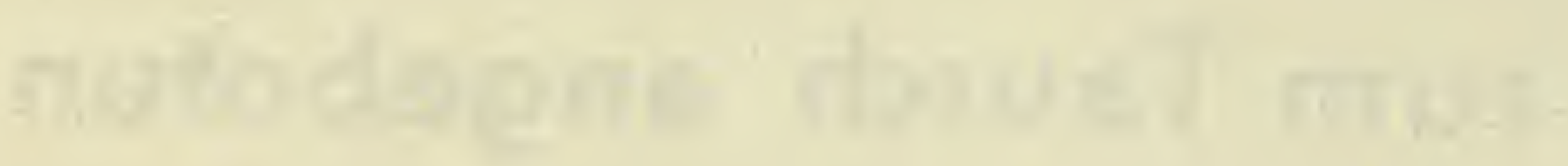

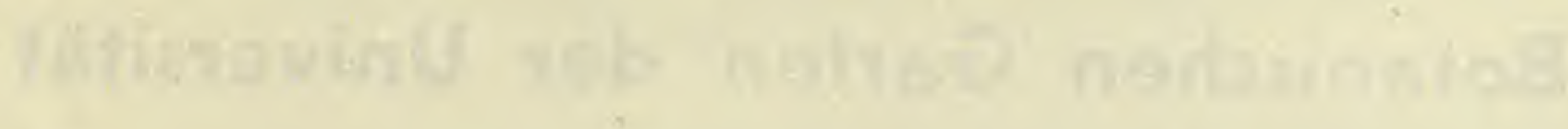

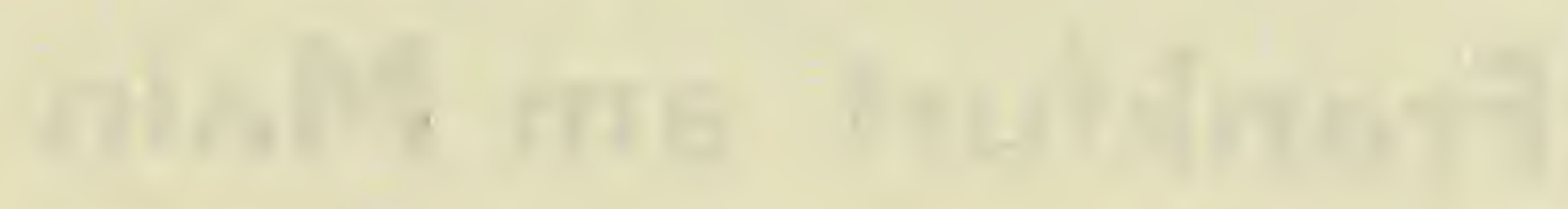




\section{Pteridophyta.}

Filices.

Cyatheaceae.

Dicksonia antarctica Labill.

Polypodiaceae.

Asplenium Belangerie Kze. nidus $\mathrm{L}$.

- trichomanes L.

Blechnum occidentale L.

Doodia aspera $\mathrm{R}$. Br.

Fadyenia prolifera Hook.

Niphobolus lingua Spring.

Polypodium aureum L.

adnescens Sw.

Meyenianum Hook.
Schizaeaceae.

Aneimia phyllitidis Sw.

- rotundifolia Schrad.

\section{Osmundaceae.}

Osmunda regalis $\mathrm{L}$.

\section{Selaginellaceae.}

Selaginella Emmeliana Van Geert.

- grandis Moore.

_- inaequalifolia Sprg.

\section{Gymnospermae.}

Coniferae.

Taxus baccata L.

- - v. erecta L.

- canadensis Willd.

- parvifolia Wender.

\section{Angiospermae. Monocotyledoneae.}

Alismataceae.

Alisma Plantago L.

\section{Amaryllidaceae.}

Crinum zeylanicum $\mathrm{L}$.

Zephyranthes Atamasco Herb.

\section{Araceae.}

Anthurium Bakeri Hook. cristallinum Lind et And. scandens Engl.

Arum italicum Mill.
Bromeliaceae.

Acanthostachys strobilacea Lk. Aechmea bromeliaefolia Bak.

Dyckia remotiflora Otto.

- sulphurea C. Koch.

Guzmania tricolor Ruiz. et Pav.

Hechtia Ghiesbreghtii Lem.

Pitcairnia undulata Schiedw.

Commelinaceae.

Palisota Barterie Hook.

Tradescantia virginiana $\mathrm{L}$. 


\section{Cyperaceae.}

Carex Grayii Carey.

- leporina L.

- vulpina L.

Cyperus adenophorus Schrad.

- alternifolius L.

- esculentus L.

- Monti L.

- natalensis Hochst.

\section{Gramineae.}

Alopecurus pratensis L.

Avena barbata. Broth.

- fatua L.

- orientalis Schreb.

- sativa L.

- strigosa Schreb.

Brachypodium distachyum Beauv. Briza maxima L.

- media L.

Bromus erectus Huds.

- purpurascens Delile.

Elymus arenarius L.

Calamagrostis epigeios Roth.

Coix Lacryma Jobi. L.

Festuca gigantea Vill.

- ovina L.

- rubra L.

Hordeum distichum L.

- hexastichum L.

- maritimum With.

- vulgare L.

- zeocriton L.

Koeleria glauca DC.

Lamarckia aurea Moench.

Melica ciliata L.

Oryza sativa L.

Panicum esculentum A.B.

- miliaceum L.

Phalaris canariensis $\mathrm{L}$.

- paradoxa L.

Poa alpina $\mathrm{L}$.

- bulbosa L.

Secale cereale $\mathrm{L}$.

Setaria italica Beauv.

viridis Beauv.

Sorghum vulgare Pers.
Stipa Calamagrostis Wahlb.

- capillata L.

- gigantea Lag.

- pennata L.

- spartea Trin.

Triticum dicoccum Schrank.

- durum Desf.

- sativum Lam.

- spelta L.

- vulgare L.

Zea Mays L.

- var. gracillima (L.) Kcke.

- - Yellow.

_- Naurottola.

- - rubra.

_ - versicolor Bonaf.

\section{Iridaceae.}

Iris aphylla L.

- germanica L.

- graminea L.

- japonica Thbg.

- juncea Poir.

- ochroleuca L.

- plicata Lam.

- pumila L.

- sibirica L.

Marica coerulea Ker.

- humilis Lodd.

- Northiana Ker. Gawl.

Sisyrinchium Bermudiana L.

- iridifolium $\mathrm{H}$. B. et $\mathrm{K}$.

\section{Juncaceae.}

Luzula albida DC.

- Forsteri DC.

- nemorosa E. Mey.

- nivea DC.

\section{Lliaceae.}

Agapanthus umbellatus L'Herit.

Allium fallax Schult.

- fistulosum L.

- globosum Bbrst.

- Moly L.

- odorum L.

- rotundum $\mathrm{L}$.

Asphodeline liburnica Rchbch.

- lutea Reichb. 
Asphodelus fistulosus L.

- tauricus Pall.

Bulbine annua Willd.

Chlorophytum amaniense Engl. usambarensis Engl.

Danae racemosa Link.

Eremurus Olgae Regel.

- robustus $\mathrm{Rgl}$.

- spectabilis Bieb.

Hemerocallis flava $L$. fulva $\mathrm{L}$.

Hypoxis villosa $\mathrm{L}$.
Kniphofia aloides Moench. Burchellii Kth.

Majanthemum bifolium

F. W. Schmidt.

Veratrum nigrum L.

Zygadenus glaucus Nutt.

Taccaceae.

Tacca macrantha W. Limpr.

Zingiberaceae.

Alpinia calcarata Rosc.

Costus igneus N. E. Brown.

Globba bulbifera Roxb.

\section{Dicotyledoneae.}

\section{Acanthaceae.}

Acanthus longifolius Poir.

- mollis L.

- squarrosa Nees.

Thunbergia alata Boj.

\section{Aizoaceae.}

Mesembrianthemum Bolusii

- cristallinum L.

- linguiforme L.

- pyropeum Haw.

Tetragonia echinata Ait.

-. expansa Murr.

\section{Amarantaceae.}

Amarantus caudatus $\mathrm{L}$.

- gangeticus L.

- paniculatus L.

Celosia argentea L.

- cristata L.

\section{Anacardiaceae.}

Rhus Cotinus L.

- vernicifera Dec.

\section{Araliaceae.}

Aralia edulis Sieb. et Zucc. japonica Thbg.

- racemosa $\mathrm{L}$.
Asclepiadaceae.

Asclepias syriaca L.

tuberosa L.

Cynanchum nigrum Pers.

Stapelia grandiflora Mass.

Vincetoxicum officinale Moench.

\section{Balsaminaceae.}

Impatiens Balsamina L.

Hook.

- Roylei Walp.

- scabrida DC.

- Sultani Hook.

\section{Begoniaceae.}

Begonia Schmidtiana Rgl.

- semperflorens Link et Otto.

\section{Berberidaceae.}

Berberis Thunbergii DC.

Podophyllum peltatum L.

Bignoniaceae.

Catalpa Kaempferi Sieb. et Zucc. Eccremocarpus scaber

Ruiz. et Pav.

Incarvillea Delavayi B. et Fr.

Jacaranda mimosaefolia D. Don.

\section{Boraginaceae.}

Alkanna tinctoria Tausch.

Anchusa officinalis L. 
Borago officinalis L.

Cerinthe major $\mathrm{L}$.

Cynoglossum officinale $\mathrm{L}$.

Echium Wildpretii H. W. Pears.

Lithospermum officinale $\mathrm{L}$.

Omphalodes amplexicaulis Lehm.

- linifolia Moench.

\section{Campanulaceae.}

Campanula alliariaefolia Willd.

- bononiensis L.

- glomerata L.

- lactiflora Bieb.

- Medium L. fl. albo.

- fl. coeruleo.

- - fl. roseo.

- fl. roseo $\mathrm{pl}$.

- persicifolia L.

- rotundifolia L.

- Trachelium L.

- turbinata Schott.

- versicolor Sibth. et Sm.

Phytheuma campanuloides Bieb.

Platycodon grandiflorum DC.

Specularia pentagonia DC.

- Speculum A. DC.

Wahlenbergia dalmatica DC.

- lobelioides Link.

\section{Capparidaceae.}

Cleome gigantea L.

- spinosa Jacq.

- violacea L.

Polanisia graveolens Rafin.

\section{Caprifoliaceae.}

Lonicera Caprifolium L.

- spinosa Jacq.

Sambucus racemosa $L$.

Viburnum Lantana L.

\section{Caryophyllaceae.}

Agrostemma Githago L.

Cucubalus baccifer L.

Dianthus barbatus L.

- caucasicus Bieb.

- fragrans Bbrst.
- frigidus Zucc.

- neglectus Lois.

- pelviformis Heuff.

- plumarius L.

- praecox Willd.

- rigidus Bieb.

- superbus L.

Gypsophila elegans M. B.

- paniculata L.

- repens L.

Lychnis chalcedonica L.

- coronaria Desr.

- flos cuculi L.

- viscaria Scop.

Melandrium album Garke.

- noctiflorum Fr.

Saponaria officinalis L.

Silene gallica $\mathrm{L}$.

- glauca Zea.

- inflata Sm.

- quinquevulnera L.

- Schafta Gmel.

-.. viridiflora $\mathrm{L}$.

- Zawadskii Herb.

Spergula arvensis L.

Tunica saxifraga Scop.

\section{Celastraceae.}

Evonymus alatus Elm.

- europaeus L.

- latifolius Mill.

\section{Chenopodiaceae.}

Atriplex rosea L.

Beta vulgaris $\mathrm{L}$.

Blitum virgatum $\mathrm{L}$.

Chenopodium Quinoa Willd.

Kochia scoparia Schrad.

Salsola collina Pall.

- Kali L.

- Soda L.

\section{Cistaceae.}

Helianthemum vulgare Gaertn.

Cneoraceae.

Cneorum tricoccon L. 
Compositae.

Achillea eupatorium Bieb. var.

Parkeri.

-- Millefolium L.

- nobilis L.

- ptarmica L.

Ageratum mexicanum Sweet.

Ammobium alatum R. Br.

Anacycius officinarum Hayne.

Anaphalis margaritaceae Benth.

Anthemis Triumfetti All. et Hook.

Arctium Lappa L.

- tomentosum Mill.

Arctotis grandis Thbg.

Arnica Chamissonis Less.

- montana L.

Artemisia absinthium L.

- dracunculus L.

- vulgaris $\mathrm{L}$.

Aster amellus L.

- linosyris Bernh.

- macrophyllus L.

- tibeticus Hook.

Brachycome iberidifolia Benth.

Buphthalmum salicifolium L.

Cacalia suaveolens L.

Calendula officinalis L.

Carlina acanthifolia All.

Catananche coerulea L.

Cenia turbinata Pers.

Centaurea atropurpurea W. et K.

- dealbata Willd.

- Rhaponticum L.

Chrysanthemum Balsamita L.

-- parthenium Bernh.

- roseum Adam.

Cirsium heterophyllum All.

Cnicus benedictus L.

Coreopsis tinctoria Nutt.

Cosmos sulphureus Cav.

Crepis aurea Reichb.

- grandiflora Tausch.

- lampsanoides Froel.

- pulchra L.

- rubra L.

- sibirica L.

Dimorphotheca pluvialis Moench.
Echinops exaltatus Schrad. ritro $\mathrm{L}$.

- sphaerocephalus L.

Erigeron bellidifolius Muhl.

- speciosus DC.

Eupatorium aromaticum L.

- Haageanum Rgl. et Kern.

- heterophyllum DC.

- janthinum Hemsl.

- purpureum L.

- var. angustifolium L.

Gaillardia aristata Pursh.

Gerbera Anandria Sch. Bip.

Helenium autumnale L.

Helianthus annuus L.

Helichrysum bracteatum Andr.

Hieracium albidum Vill.

- amplexicaule L.

- aurantiacum L.

- bifurcum Bbrst.

- foliosum $\mathrm{W}$. et $\mathrm{K}$.

- Heldreichii Boiss.

- umbellatum L.

- villosum L.

Inula britannica $\mathrm{L}$.

- helenium L.

- thapsoides Spr.

Lactuca perennis L.

- virosa L.

Leontopodium alpinum Cass.

Ligularia sibirica DC.

Matricaria Chamomilla L.

Mulgedium macrophyllum DC.

Onopordon illyricum L.

Parthenium integrifolium L.

Petasites vulgaris Desf.

Picris echioides L.

Rhagadiolus stellatus Gaertn.

Rudbeckia laciniata L.

- purpurea L.

- triloba L.

Sanvitalia procumbens Lam.

Scorconera hispanica L.

Senecio artemisiaefolius Pers.

- clivorum Max.

- Doria L.

- macrophyllus Bbrst.

Serratula tinctoria L.

Silybum Marianum Gaertn. 
Solidago canadensis L.

- lanceolata Burm.

- serotina Ait.

- Virgaurea L.

Sonchus palustris L.

Tagetes erecta $\mathrm{L}$.

- patula L.

Tanacetum vulgare L.

Telekia speciosa Baumg.

Xanthium strumarium L.

Zinnia elegans Jacq.

- Haageana Klotsch.

\section{Convolvulaceae.}

Ipomoea purpurea Lam.

- sibirica Pers.

- splendens L.

\section{Cornaceae.}

Cornus mas L.

Cornus sibirica L.

\section{Cruciferae.}

Alliaria officinalis Andrz.

Alyssum saxatile L.

Arabis alpina L.

Barbarea vulgaris $\mathrm{R}$. $\mathrm{Br}$.

Berteroa incana DC.

Biscutella auriculata L.

Brassica oleracea L.

- napus L.

Bunias orientalis $\mathrm{L}$.

Cakile maritima Scop.

Camelina sativa Crantz.

Cardamine pratensis $\mathrm{L}$.

Cheiranthus Cheiri L.

Cochlearia officinalis $\mathrm{L}$.

Crambe cordifolia Stev.

Erysimum Perofskianum

Hesperis matronalis L.

Iberis amara L.

- oderata L.

- pinnata L.

- sempervirens L.

- umbellata L.

Isatis tinctoria $\mathrm{L}$.
Lepidium campestre $\mathrm{R}$. $\mathrm{Br}$.

- ruderale $\mathrm{L}$.

- sativum L.

Lunaria annua $\mathrm{L}$.

- rediviva $\mathrm{L}$.

Myagrum perfoliatum L.

Sinapis alba L.

Sisymbrium officinale Scop.

- strictissimum L.

Vesicaria urtriculata DC.

\section{Cucurbitaceae.}

Bryonia dioica Jacq.

Cyclanthera explodens Naud.

- pedata Naud.

Ecballium Elaterium Rich.

Lagenaria vulgaris Ser.

Sicyos angulata L.

\section{Datiscaceae.}

Datisca cannabina L.

\section{Dipsacaceae.}

Cephalaria tatarica Schrad.

Dipsacus Fullonum L.

Knautia arvensis Coult.

Morina longifolia Wall.

Scabiosa atropurpurea L.

- caucasica Bieb.

- suaveolens Desf.

Succisa pratensis Moench.

\section{Elaeagnaceae.}

Elaeagnus longipes A. Gr.

\section{Euphorbiaceae.}

Euphorbia Esula L.

- Lathyris L.

- Myrsinites L.

Mercurialis perennis $\mathrm{L}$.

Ricinus communis L.

\section{Frankeniaceae.}

Frankenia pulverulenta L.

\section{Gentianaceae.}

Erythraea Centaurium Pers. linariaefolia Wittr. 
Gentiana acaulis L.

- asclepiadea L.

- lutea L.

- septemfida Pall.

- tibetica King.

\section{Geraniaceae.}

Erodium cicutarium L'Hérit. gruinum Ait.

Geranium sanguineum L.

- silvaticum L.

Pelargonium odoratissimum

Solond.

- tomentosum Jacq.

\section{Gesneraceae.}

Achimenes coccinea Pers.

- hirsuta DC.

- patens Benth.

Besleria grandiflora H.B.

Klugia Notoniana DC.

Streptocarpus Kirkii Hook.

Wendlandii Hort. Dammann.

\section{Globuíariaceae.}

Globularia cordifolia L.

Globularia trichosantha Fisch et Mey.

- vulgaris L.

\section{Hydrophyllaceae.}

Nemophila maculata Benth. Phacelia campanularia A. Gray.

- congesta Hook.

- platycarpa Spr.

- tanacetifolia Benth.

Whitlavia Gray.

\section{Labiatae.}

Betonica officinalis L. stricta Ait.

Calamintha Clinopodium Benth. officinalis Moench.

Coleus Rehneltianus Berger.

Dracophalum peregrinum L.

Hyssopus officinalis L.
Lavandula officinalis Chaix. vera $D C$.

Leonurus Cardiaca L.

Lophantus anisatus Benth.

Lycopus europaeus L.

- exaltatus L.

Marrubium candidissimum L.

Melissa officinalis L.

Mentha piperita L.

- viridis $\mathrm{L}$.

Monarda Clinopodia L.

- didyma L. fistulosa $\mathrm{L}$.

Ocimum Basilicum L.

Origanum majorana L.

Phlomis tuberosa L.

Physostegia virginiana Benth.

Preslia cervina Fres.

Salvia Aethiopis L.

- candidissima Vahl.

- dumetorum Andrz.

- Horminum L.

- pratensis L.

- Sclarea L.

- splendens Sellow.

- silvestris L.

- verticillata $\mathrm{L}$.

Satureja hortensis L.

Scutellaria alpina $\mathrm{L}$.

Sideritis dasygnaphala Clos.

Stachys germanica L.

- hirsuta H. B. et K.

- iberica Bieb.

- silvatica L.

Teucrium Chamaedrys L.

Thymus serpyllum L.

\section{Leguminosae.}

Apios tuberosa Mch. Astragalus Cicer L.

- glycyphyllos L.

Baptisia australis R. Br.

Caragana arborescens Lam.

Cercis Siliquastrum L.

Cicer arietinum $\mathrm{L}$.

Clianthus Dampieri A. Cunn.

Colutea arborescens L.

- istria Mill.

- orientalis Lam. 
Desmodium gyrans DC.

Dolichos catjang L.

- lablab L.

- ornatus Wall.

Galega officinalis L.

Genista sagittalis L.

Glycyrrhiza echinata L.

Gymnocladus canadensis Lam.

Kennedya prostrata $\mathrm{R}$. Br. Laburnum vulgare J. Presl.

Latyrus clymenum L.

- latifolius L.

- Nissolia L.

- odoratus L.

- roseus Stev.

- tingitanus L.

- vernus Bernh.

Lotus ornithopodioides L.

Lourea vespertilionis Desv.

Lupinus albus L.

- Barkeri Ldl.

- Cruckshanksii Hook.

- Douglasii Agardh.

- elegans H. B. et K.

- Hartwegii Ldl.

- polyphyllus Ldl.

Medicago intertexta Willd.

- orbicularis All.

Melilotus officinalis Lam.

Mimosa pudica L.

Onobrychis Caput galli Lam.

- crista galli Lam.

Ononis Natrix L.

Orobus vernus L.

Phaseolus multiflorus Willd.

- vulgaris L.

Rhynchosia phaseoloides DC.

Securigera Coronilla DC.

Soja hispida Moench.

Trifolium elegans Savi.

- pannonicum Jacq.

Vicia Faba L.

- var. equina L.

- ferruginea Bess.

- narbonensis L.

- sativa L.

Vigna glabra Savi.

\section{Linaceae.}

Linum alpinum $\mathrm{L}$. angustifolium Huds.
- austriacum L.

- flavum L.

- grandiflorum Desf.

- hirsutum L.

- narbonnense L.

- perenne L.

- usitatissimum L.

\section{Loasaceae.}

Blumenbachia Hieronymi Urb.

Caiophora lateritia Benth.

Loasa tricolor Lindl.

\section{Lobeliaceae.}

Lobelia fulgens Willd.

- inflata $\mathrm{L}$.

- syphilitica L.

- triquetra L.

- urens L.

\section{Loganiaceae.}

Buddleia Lindleyana Fort.

\section{Lythraceae.}

Heimia salicifolia Link et Otto. Lythrum Salicaria L.

\section{Malvaceae.}

Abutilon tiliaefolium Sweet.

Althaea officinalis $\mathrm{L}$.

- rosea Cav.

Anoda hastata Cav.

Gossypium herbaceum L.

Hibiscus Manihot L.

- syriacus L.

- Trionum L.

Kitaibelia vitifolia Willd.

Lavatera arborea L.

- trimestris L.

Malachra alceaefolia Jacq.

Malope trifida Cav.

Malva crispa L.

- silvestris L.

Pavonia spinifex Cav.

Sida Abutilon L.

Sparmannia palmata Hort.

Urena lobata L. 


\section{Magnoliaceae.}

Magnolia tripetala $\mathrm{L}$.

- Yulan Desf.

\section{Martyniaceae.}

Martynia lutea Lindl. - proboscidea Glox.

\section{Melastomataceae.}

Bertolonia marmorata Naud.

Centradenia grandifolia Endl.

\section{Moraceae.}

Cannabis sativa $\mathrm{L}$.

Ficus edulis Burm.

- religiosa L.

Humuius Lupulus L.

\section{Myrsinaceae.}

Ardisia crenulata Vent.

- humilis Vahl.

\section{Myrtaceae.}

Callistemon coccineus F. Muell. linearis DC.

Eugenia myrtifolia Sims.

Leptospermum flavescens Sm.

Myrtus communis L.

\section{Nolanaceae.}

Nolana prostrata L.

\section{Nyctaginaceae.}

Mirabilis dichotoma $\mathrm{L}$.

- Froebelii Gr.

- Jalapa L.

- longiflora L.

Oxybaphus nyctagineus Sweet.

\section{Ochnaceae.}

Ochna multiflora DC.

\section{Oenotheraceae.}

Clarkia elegans Dougl.

- pulchella Pursh.
Cuphea lanceolata Ait.

- Llavea Lindl.

- procumbens Cav.

Epilobium angustifolium L.

- Dodonaei Vill.

- roseum Schreb.

Fuchsia procumbens R. Cunn. Gaura Lindheimeri Engelm. Jussieua salicifolia H. B.

Lopezia racemosa Cav.

Oenothera acaulis Cav.

- Drummondii Hook.

- Fraseri Pursh.

- fruticosa L.

- glauca Michx.

- grandiflora Lam.

- Lamarkiana Ser.

- Lindleyana Steud.

- muricata L.

\section{Oleaceae.}

Jasminum odoratissimum L. Ligustrum japonicum Hort. Syringa Emodi Wall.

\section{Orobanchaceae.}

Orobanche Hederae Duby.

- lucorum A. Br.

- ramosa L.

\section{Oxalidaceae.}

Biophytum dormiens G. Don.

- sensitivum DC.

\section{Papaveraceae.}

Argemone mexicana L.

Chelidonium majus L.

- var. laciniatum Mill.

Eschscholzia californica Cham.

Glaucium corniculatum Curt.

- luteum Scop.

Papaver alpinum L.

- bracteatum Lindl.

- caucasicum M. B.

- dubium L.

- Heldreichii Boiss.

- lateritium C. Koch.

- nudicaule L.

- orientale L.

- Rhoeas L.

- somniferum L. 
Phytoloccaceae.

Petiveria alliacea $\mathrm{L}$.

Phytolacca decandra L.

Rivina aurantiaca Warsz.

- humilis L.

laevis $\mathrm{L}$.

\section{Plantaginaceae.}

Plantago arenaria Waldst. et Kit.

Cynops L.

- lanceolata L.

- major L.

- major L. var. monstrosa.

-. maritima L.

- media L.

- serpentina All.

- Psyllium L.

\section{Plumbaginaceae.}

Armeria alpina Willd.

maritima Willd.

- plantaginea Willd.

Statice Gmelini Willd.

- Limonium L.

\section{Polemoniaceae.}

Cobaea scandens Cav.

Collomia grandiflora Dougl.

Gilia capitata Sims. multicaulis Benth.

Phlox Drummondii Hook.

Polemonium coeruleum L.

- grandiflorum Benth.

- mexicanum Cerv.

- pauciflorum S. Wats.

\section{Polygonaceae.}

Atraphaxis lanceolata Bunge.

Emex spinosa Campd.

Fagopyrum tataricum Gaertn.

Oxyria digyna Hill.

Polygonum Bistorta L.

orientale L.

Rheum Emodi Wall.

- officinale Baill.

- palmatum L. var. tanguticum.

- undulatum L.

Rumex acetosa L.

- alpinus L.

- patientia L.

- scutatus L.

\section{Portulacaceae.}

Calandrinia compressa Schrad.

Claytonia perfoliata Donn.

Portulaca oleracea L.

Ullicus tuberosus Cald.

\section{Primulaceae.}

Androsace primuloides Duby. sarmentosa Wall.

Lysimachia heterophylla Michx. vulgaris $\mathrm{L}$.

Primula acaulis Hill. Auricula L.

- Bulleyana G. Forrest.

- denticulata Sm.

- malacoides F.

- officinalis Jacq.

- rosea Royle.

- saxatilis Kom.

Soldanella alpina L.

\section{Ranunculaceae.}

Aconitum Anthora L.

- Lycoctonum L.

Napellus L.

Anemone Pulsatilla L.

Aquilegia alpina L.

- coerulea James.

- formosa Fisch.

- Skinneri Hook.

- vulgaris L.

Clematis Flammula L. integrifolia $\mathrm{L}$.

Delphinium elatum L.

- formosum Boiss. et Huet.

- grandiflorum L.

- Maackianum Rgl.

- Staphysagria L.

Myosurus minimus L.

Nigella arvensis L.

- damascena L.

- sativa L.

Paeonia officinalis $\mathrm{L}$.

Pulsatilla vulgaris Mill.

Ranunculus acer L.

- aconitifolius L.

- asiaticus L.

Thalictrum elatum Jacq.

Trollius europaeus L. 
Resedaceae

Reseda alba L.

- complicata Bory.

- cristallina Webb. et Berth.

- glauca L.

- media Lag.

- odorata L.

- Phyteuma L.

- lutea L.

- luteola L.

\section{Rhamnaceae}

Ceanothus azureus Desf.

\section{Rosaceae}

Acaena sanguisorbae Vahl.

Cotoneaster horizontalis Decne. Crataegus pyracantha Medic.

Dryas octopetala L.

Exochorda Alberti Rgl.

- grandiflora Lindl.

Geum coccineum Sibth. et Sm.

- montanum L.

- rivale $\mathrm{L}$.

Mespilus germanica L.

Neviusia alabamensis A. Gr.

Photinia laevis DC.

Potentilla atrosanguinea Lodd.

- grandiflora L.

- norvegica L.

- rupestris L.

- Salesovii Steph.

- verna L.

Rhodotypos kerrioides S. et Z.

Sorbus Aucuparia L.

Spiraea Douglasii Hook.

- filipendula L.

\section{Rubiaceae}

Asperula odorata L.

- tinctoria L.

Crucianella aegyptiaca L.

Galium boreale L.

- purpureum L.

- rigidum Ait.

- saccharatum All.

- tricorne Stokes.

Rubia tinctorum L.

\section{Rutaceae}

Dictamnus albus L.

Erythrochiton brasiliensis Nees. et Mart.

Ruta graveolens L.

\section{Sapindaceae}

Koelreuteria paniculata Laxm.

\section{Saxifragaceae}

Heuchera americana L.

- micrantha Dougl.

- sanguinea Engelm.

Rodgersia aesculifolia Batalin.

- podophylla A. Gray.

Saxifraga Aizoon Jacq.

- Cotyledon L.

- crassifolia L.

- granulata L.

- hypnoides L.

- muscoides Wulf.

- pulchella Don.

- Steinmanni Tausch.

- tricuspidata Rott.

- trifurcata Schrad.

- umbrosa L.

Tiarella cordifolia L.

\section{Scrophulariaceae}

Antirrhinum majus L.

- Orontium L.

Digitalis ambigua Murr.

- ferruginea L.

- lanata Ehrh.

- lutea L.

- nervosa Steud.

- purpurea L.

Erinus alpinus L.

Gratiola officinalis L.

Linaria bipartita Willd.

- triphylla Mill.

- vulgaris Mill.

Maurandia scandens A. Gray.

Mimulus cardinulis Dougl.

- luteus L.

- moschatus Dougl.

- ringens $\mathrm{L}$.

Nemesia floribunda Lehm.

- strumosa Benth. 
Paulownia imperialis Sieb. et Zucc.

Pentstemon barbatus Roth.

- confertus Dougl.

- gentianoides Poir.

- glaucus Graham.

- hirsutus Willd.

Rehmannia angulata Hemsl.

Scrophularia alata Gil.

- Neesii Wirtg.

- nodosa L.

- vernalis L.

Tetranema mexicanum Benth.

Torenia Fournieri Linden.

Verbascum Blattaria L.

- Lychnitis L.

- nigrum L.

- phlomoides L.

- phoeniceum L.

- thapsiforme Schrad.

- Thapsus L.

Veronica officinalis L.

- urticaefolia L.

\section{Solanaceae.}

Atropa belladonna L.

Capsicum annuum L.

Datura laevis $\mathrm{L}$.

- quercifolia H. B. K.

- Stramonium L.

- Tatula L.

Hyoscyamus albus L.

- niger L.

Lycopersicum esculentum Mill.

Nicandra physaloides Gaertn.

Nicotiana alata Link. et Otto.

- longiflora Cav.

- rustica L.

- Sanderae Ht.

- virginica Agarth.

Physalis alkekengi L.

Schizanthus pinnatus

Ruiz. et Pav.

Solanum Capsicastrum Lk.

- nigrum L.

\section{Staphyleaceae.}

Staphylea colchica Stev. - pinnata L.
Styracaceae.

Halesia hispida Gard. Chron. - tetraptera L.

\section{Tropaeolaceae.}

Tropaeolum aduncum Sm.

- Lobbianum Ht.

- majus L.

- minus L.

- peregrinum L.

\section{Ulmaceae.}

Celtis occidentalis L.

\section{Umbelliferae.}

Aegopodium podagraria L. Aethusa cynapium L. Anethum graveolens L. Anthriscus cerefolium Hoffm. Archangelica officinalis Hoffm. Astrantia Biebersteinii Fisch.

- helleborifolia Salisb.

- major L.

Bupleurum fruticosum L.

- graminifolium Vahl.

- junceum L.

- longifolium L.

- polyphyllum Ledeb.

- ranunculoides L.

- rotundifolium L.

- salicifolium Soland.

Carum carvi L.

Chaerophyllum aromaticum $\mathrm{L}$.

- bulbosum L.

Cicuta virosa L.

Conium maculatum L.

Coriandrum sativum L.

Daucus Carota L.

Eryngium agavifolium Griseb.

- alpinum L.

- amethystinum L.

- eburneum Decne.

- planum L.

Foeniculum vulgare Mill.

Heracleum Lehmannianum. Bunge.

-sphondylium L.

Hydrocotyle vulgaris L.

Imperatoria Ostruthium L.

Laserpitium Panax Gouan. 
Levisticum officinale Koch.

Libanotis montana Crantz. Meum athamanticum Jacq. Myrrhis odorata Scop.

Oenanthe Lachenalii Gmel.

Pastinaca sativa $L$.

Peucedanum officinale $\mathrm{L}$.

Pimpinella magna L.

- rotundifolia Bbrst.

- Saxifraga L.

Sanicula europaea L.

Scandix Pecten-Veneris L.

Siler aquilegifolium Gaertn.

Sium Sisarum L.

Smyrnium Olusatrum L.

- perfoliatum L.

Tordylium syriacum L.

\section{Urticaceae.}

Laportea moroides Wedd.

Parietaria officinalis L.

Pilea Spruceana Wedd.

Procris australis Spr.

Urtica baccifera L.

- dioica L.

- moroides Cunningh.

- pilulifera L.

\section{Valerianaceae.}

Centranthus ruber DC.

Fedia cornucopiae Lange.

Patrinia gibbosa Maxim.

Valeriana dioica L.

- officinalis L.

- Phu L.

- sambucifolia Mikan.

\section{Verbenaceae.}

Lippia citriodora $\mathrm{H}$. B. et Kth. Verbena officinalis $\mathrm{L}$.

- urticifolia L.

- venosa Gill. et Hook.

Vitex Agnus-castus L.

\section{Violaceae.}

Viola cornuta L.

- tricolor L.

\section{Zygophyllaceae.}

Peganum Harmala L.

Tribulus terrestris $\mathrm{L}$.
Prof. Dr. Peter Stark

Direktor.
Rudolf Günther

Gartenoberinspektor. 



\section{SAMEREIEN \\ zum Tausch angeboten}

vom

Botanischen Garten der Universitäł

Frankfurt am Main

J a n a r 1930

\section{JAN 171930}


$x^{2}+x^{2}=x^{2}=$ 


\section{Pteridophyta}

\section{Filcales}

\section{Cyatheaceae}

Dicksonia antarctica Labill.

\section{Polypodiaceae}

Asplenium Belangerie Kze.

- nidus $\mathrm{L}$.

Blechnum occidentale L.

Doodia aspera $\mathrm{R}$. Br.

Fadyenia prolifera Hook.

Niphobolus lingua Spring.

Polypodium aureum L.

- adnescens Sw.

- Meyenianum Hook.

\section{Schizaeaceae}

Aneimia phyllitidis Sw.

- rotundifolia Schrad.

\section{Osmundaceae}

Osmunda regalis $\mathrm{L}$.

\section{Selaginellaceae}

Selaginella Emmeliana Van Geert.

- grandis Moore.

- inaequalifolia Sprg.

\section{Angiospermae Monocotyledoneae}

\section{Alismafaceae}

Alisma Plantago L.

\section{Amaryllidaceae}

Crinum zeylanicum $\mathrm{L}$.

Zephyranthes Atamasco Herb.

\section{Araceae}

Anthurium Bakeri Hook.

- scandens Engl.

\section{Bromeliaceae}

Acanthostachys strobilacea Lk.

Aechmea bromeliaefolia Bak.

Dyckia remotiflora Otto

- sulphurea C. Koch.

Hechtia Ghiesbreghtii Lem.

Pitcairnia undulata Schiedw.

\section{Commelinaceae}

Palisota Barterie Hook.

Tradescantia virginiana $\mathrm{L}$.

\section{Cyperaceae}

Carex Grayii Carey.

Cyperus alternifolius $\mathrm{L}$.

- adenophorus Schrad.

- esculentus L.

- Monti L.

- natalensis Hochst.

\section{Gramineae}

Avena barbata. Broth.

— fatua L.

- orientalis Schreb.

- sativa L.

- strigosa Schreb. 
Boissiera bromoides Hochst. et

Steud.

Brachypodium distachyum Beauv. Briza maxima L.

" media L.

Bromus ereetus Huds.

- purpurascens Delile.

Calamagrostis epigeios Roth.

Coix Lacryma Jobi. L.

Festuca gigantea Vill.

- ovina L.

- rubra $\mathrm{L}$.

Hordeum distichum L.

- hexastichum L.

- maritimum With.

- vulgare $\mathrm{L}$.

- zeocriton L.

Koeleria glauca DC.

Lamarckia aurea Moench.

Melica ciliata L.

Oryza sativa $L$.

Panicum esculentum A.B. " miliaceum $\mathrm{L}$.

Phalaris canariensis L.

- paradoxa L.

Poa alpina L.

- bulbosa L.

Secale cereale L.

Setaria italica Beauv.

- viridis Beauv.

Sorghum vulgare Pers. var. nig-

Stipa Calamagrostis Wahlb. rum Kcke.

- capillata L.

- pennata L.

Triticum dicoceum Schrank.

- durum Desf.

- monococcum L.

- sativum Lam.

- spelta L.
-- vulgare $\mathrm{L}$.

Zea Mays L.

-. - var. gracillima (L.) Kcke.

__ _ Yellow.

- Naurottola.

rubra.

- - Tubra.

—— versicolor Bonaf.

\section{Iridaceae}

Iris aphylla L.

- germanica L.

- graminea $\mathbf{L}$.

- japonica Thbg.

- juncea Poir.

- ochroleuca L.

- plicata Lam.

- pumila L.

- sibirica L.

Marica coerulea Ker.

- humilis Lodd.

- Northiana Ker. Gawl.

Sisyrinchium Bermudiana L.

— iridifolium H. B. et $\mathbf{K}$.

\section{Juncaceae}

Luzula albida DC.

- Forsteri DC.

- nemorosa E. Mey.

- nivea DC.

\section{Liliaceae}

Agapanthus umbellatus L'Herit.

Allium fallax Schult.

- fistulosum L.

- globosum Bbrst.

- Moly L.

- rotundum L.

Asphodelus fistulosus L.

- tauricus Pall.

Asphodeline liburnica Rchbch. 
- lutea Rchbch.

Bulbine annua Willd.

Chlorophytum amaniense Engl.

- usambarensis Engl.

Danae racemosa Link

Eremurus Olgae Regel

- robustus Rgl.

- spectabilis Bieb.

Hypoxis villosa $\mathrm{L}$.

Kniphofia aloides Moench.

- Burchellii Kth.
Majanthemum bifolium

F. W. Schmidt.

Veratrum nigrum $L$.

Zygadenus glaucus Nutt.

\section{Taccaceae}

Tacca macrantha W. Limpr.

\section{Zinglberaceae}

Alpinia calcarata Rosc.

Costus igneus N. E. Brown.

Globba bulbifera Roxb.

\section{Dicotyledoneae}

\section{Acanthaceae}

Acanthus longifolius Poir.

- mollis L.

- squarrosa Nees

\section{Aizoaceae}

Mesembrianthemum crystallinum

- pyropeum Haw.

Tetragonia echinata Ait.

- expansa Murr.

\section{Amarantaceae}

Amarantus caudatus L.

- gangeticus L.

- paniculatus L.

\section{Anacardiaceae}

Rhus Cotinus L.

- vernicifera Dec.

\section{Arallaceae}

Aralia edulis Sieb. et Zucc.

- japonica Thbg.

- racemosa $\mathrm{L}$.

\section{Asclepiadaceae}

Asclepias syriaca L.

- tuberosa L.

Cynanchum nigrum Pers.

Stapelia grandiflora Mass.

\section{Balsaminaceae}

L. Impatiens Balšamina L.

- Roylei Walp.

- scabrida DC.

\section{Basellaceae}

Basella alba L.

- rubra $\mathrm{L}$.

\section{Begonlaceae}

Begonia Schmidtiana Rgl.

- semperflorens Link et Otto.

\section{Berberidaceae}

Berberis Thunbergii DC.

Podophyllum peltatum L.

\section{Bignoniaceae}

Catalpa Kaempferi Sieb. et Zucc. 
Eccremocarpus seaber Ruizet Pav. Incarvillea Delavayi B. et Fr.

\section{Boraginaceae}

Anchusa officinalis L.

Borago officinalis $\mathrm{L}$.

Cerinthe major $\mathbf{L}$.

Cynoglossum officinale $\mathbf{L}$.

Echium Wildpretii H. W. Pearson.

Lithospermum officinale L.

Omphalodes amplexicaulis Lehm. - linifolia Moench.

\section{Campanulaceae}

Campanula alliariaefolia Willd.

- bononiensis $\mathrm{L}$.

- glomerata L.

- lactiflora Bieb.

- Medium L. fl. albo.

- f fl. coeruleo.

- - fl. roseo.

- fl. roseo $\mathrm{pl}$.

- persicifolia L.

- rotundifolia $\mathrm{L}$.

- Trachelium L.

- turbinata Schott.

- versicolor Sibth. et Sm.

Phyteuma campanuloides Bieb.

Platycodon grandiflorum DC.

Specularia pentagonia DC.

- Speculum A. DC.

Wahlenbergia dalmatica DC.

- lobelioides Link.

\section{Capparidaceae}

Cleome gigantea $\mathrm{L}$.

- spinosa Jacq.

- violacea L.

Polanisia graveolens Rafin.
Caprifoliaceae

Lonicera Caprifolium L.

- spinosa Jacq.

Sambucus racemosa $L$.

Viburnum Lantana L.

\section{Caryophyllaceae}

Agrostemma Githago L.

Dianthus barbatus L.

- deltoides L.

- fragrans Bbrst.

- frigidus Zucc.

- neglectus Lois.

- pelviformis Heuff.

- plumarius L.

Gypsophila elegans M. B.

- paniculata $\mathrm{L}$.

- repens $\mathrm{L}$.

Lychnis chalcedonica L.

- coronaria Desr.

- flos cuculi L.

- viscaria Scop.

Melandrium album Garke.

- noctiflorum Fr.

Saponaria officinalis L.

Silene gallica L.

- glauca Zea.

- inflata Sm.

- quinquevulnera L.

- Schafta Gmel.

- viridiflora L.

- Zawadskii Herb.

Tunica saxifraga Scop.

\section{Celastraceae}

Evonymus europaeus L. latifolius Mill. 


\section{Chenopodiaceae}

Atriplex rosea $\mathrm{L}$.

Beta vulgaris $L$.

Blitum virgatum $\mathrm{L}$.

Chenopodium Quinoa Willd.

Kochia scoparia Schrad.

Salsola collina Pall.

- Kali L.

- Soda L.

\section{Cistaceae}

Helianthemum vulgare Gaertn.

\section{Compositae}

Achillea eupatorium Bieb. var.

Parkeri.

- Millefolium L.

- nobilis L.

- ptarmica L.

Ageratum mexicanum Sweet.

Ammobium alatum R. Br.

Anacyclus officinarum Hayne

Anaphalis margaritacea Benth.

Anthemis Triumfetti All.

Aretium Lappa L.

- tomentosum Mill.

Arnica Chamissonis Less.

- montana L .

Artemisia absinthium L.

- dracunculus L. vulgaris $\mathrm{L}$.

Aster amellus L.

- linosyris Bernh.

- macrophyllus $\mathbf{L}$.

- tibeticus Hook.

Brachycome iberidifolia Benth.

Buphthalmum salicifolium L.

Cacalia suaveolens L.

Calendula officinalis $\mathbf{L}$.
Carlina acanthifolia All.

Catananche coerulea $\mathrm{L}$.

Cenia turbinata Pers.

Centaurea atropurpurea W. et K.

- dealbata Willd.

- Rhaponticum L.

Chrysanthemum Balsamita L.

- parthenium Bernh.

- roseum Adam

Cirsium heterophyllum All.

Cnicus benedictus L.

Coreopsis tinctoria Nutt.

Cosmos bipinnatus Cav.

Crepis aurea Reichb.

- grandiflora Tausch.

- lampsanoides Froel.

- pulchra L.

- rubra $\mathrm{L}$.

- sibirica L.

Dimorphotheca pluvialis Moench.

Echinops exaltatus Schrad.

- ritro $\mathrm{L}$.

- sphaerocephalus L.

Erigeron bellidifolius Muhl.

- speciosus DC.

Eupatorium aromaticum L.

- cannabinum L.

- Haageanum Rgl. et Kern.

- heterophyllum Dec.

- janthinum Hemsl.

- purpureum L.

- var. angustifolium L.

Gaillardia aristata Pursh.

Gerbera Anandria Sch. Bip.

Helenium autumnale L.

Helianthus annuus L.

Helichrysum bracteatum Andr.

Hieracium albidum Vill.

- amplexicaule $\mathbf{L}$.

- aurantiacum L. 
- bifurcum Bbrst.

- foliosum W. et K.

- Heldreichii Boiss.

- umbellatum L.

- villosum L.

Inula britannica $\mathrm{L}$.

- helenium L.

- thapsoides Spr.

Lactuca perennis L.

- virosa L.

Leontopodium alpinum Cass.

Ligularia sibirica DC.

Matricaria Chamomilla L.

Mulgedium macrophyllum DC.

Onopordon illyricum L.

Parthenium integrifolium $\mathrm{L}$.

Picris echioides $\mathrm{L}$.

Rudbeckia laciniata L.

- purpurea L.

Sanvitalia procumbens Lam.

Scorconera hispanica $L$.

Senecio artemisiaefolius Pers.

- clivorum Max.

- Doria L.

- macrophyllus Bbrst.

Serratula tinctoria L.

Silybum Marianum Gaertn.

Solidago canadensis L.

- lanceolata Burm.

- serotina Ait.

- Virgaurea L.

Sonchus palustris L.

Spilanthes Acmella Murr.

- alba L'Herit.

- oleracea L.

Tagetes erecta $\mathbf{L}$.

- patula L.

Tanacetum vulgare $L$.

Telekia speciosa Baumg.

Xanthium spinosum L.
- strumarium L.

Zacintha verrucosa Gaertn.

Zinnia elegans Jacq.

- Haageana Klotsch.

\section{Convolvulaceae}

Ipomoea purpurea Lam.

- splendens L.

\section{Cornaceae}

Cornus mas L.

$"$ sibirica L.

\section{Cruciferae}

Alyssum saxatile L.

Arabis alpina L.

Barbarea vulgaris $\mathrm{R}$. $\mathrm{Br}$

Biscutella auriculata L.

Brassica oleracea $\mathrm{L}$.

- napus L.

Bunias orientalis L.

Camelina sativa Crantz.

Cardamine pratensis $\mathbf{L}$.

Cheiranthus Cheiri L.

Crambe cordifolia Stev.

Draba alpina L.

Eruca sativa Mill.

Hutchinsia brevicaulis Hoppe.

Iberis amara $\mathbf{L}$.

- odorata L.

- pinnata L.

- sempervirens L.

- umbellata L.

Isatis tinctoria $\mathbf{L}$.

Lepidium campestre R. Br.

- ruderale $L$.

- sativum L.

Lunaria annua $\mathrm{L}$.

- rediviva $\mathrm{L}$.

Myagrum perfoliatum L. 
Sinapis alba $\mathbf{L}$.

Sisymbrium officiale Scop.

Vesicaria utriculata DC.

\section{Cucurbitaceae}

Bryonia dioica Jacq.

Cyclanthera explodens Naud.

- pedata Naud.

Ecballium Elaterium Rich.

Sicyos angulata $\mathrm{L}$.

\section{Datiscaceae}

Datisca cannabina L.

\section{Dipsacaceae}

Cephalaria tatarica Schrad.

Dipsacus Fullonum L.

Knautia arvensis Coult.

Morina longifolia Wall.

Scabiosa atropurpurea L.

- caucasica Bieb.

\section{Elaeagnaceae}

Elaeagnus longipes A. Gr.

\section{Euphorblaceae}

Euphorbia Esula L.

- Lathyris L.

- Myrsinites L.

Mercurialis perennis L.

Ricinus communis L.

\section{Frankeniaceae}

Frankenia pulverulenta L.

\section{Gentianaceae}

Erythraea Centaurium Pers.

- linariaefolia Wittr.

Gentiana acaulis $\mathbf{L}$.

- asclepiadea L.
- cruciata L.

- tibetica King.

\section{Geraniaceae}

Erodium cicutarium L'Hérit.

- gruinum Ait.

Geranium sanguineum L.

- silvaticum L.

Pelargonium tomentosum Jacq.

\section{Gesneraceae}

Achimenes coccinea Pers.

- hirsuta DC.

- patens Benth.

Streptocarpus Kirkii Hook

- Wendlandii Hort. Dammann

\section{Globulariaceae}

Globularia trichosantha Fisch et Mey.

- vulgaris $\mathrm{L}$.

\section{Hydrophyllaceae}

Nemophila maculata Benth.

Phacelia campanularia A. Gray,

- congesta Hook.

- platycarpa Spr.

- tanacetifolia Benth.

- Whitlavia Gray.

\section{Labiatae}

Betonica officinalis L.

- stricta Ait.

Calamintha Clinopodium Benth.

- officinalis Moench.

Coleus Rehneltianus Berger.

- thyrsoideus Baker.

Dracocephalum peregrinum L.

Hyssopus officinalis L.

Lavandula officinalis Chaix.

- vera DC. 
Leonarus Cardiaca L.

Lophantus anisatus Benth.

Marrubium candidissimum L.

Melissa officinalis L.

Mentha piperita L.

- viridis $\mathrm{I}$.

Monarda Clinopodia L.

- didyma L.

- fistulosa L.

Ocimum Basilicum L.

Phlomis tuberosa L.

Physostegia virginiana Benth.

Salvia Aethiopis L.

- candidissima Vahl.

- dumetorum Andrz.

- Horminum L.

- pratensis L.

- Sclarea L.

- splendens Sellow

- silvestris L.

- verticillata $L$.

Scutellaria lupulina L.

Stachys germanica L.

- hirsuta H. B. et K.

\section{Leguminosae}

Apios tuberosa Mch.

Astragalus Cicer L.

- glycyphyllos L.

Baptisia australis R. Br.

Caragana arborescens Lam.

Cassia floribunda Cav.

Cercis Siliquastrum L.

Cicer arietinum L.

Clianthus Dampieri A. Cunn.

Colutea arborescens L.

- istria Mill.

- orientalis Lam.

Dolichos eatjang L.

- lablab L.
- ornatus Wall.

Galega officinalis L.

Genista sagittalis L.

Glycyrrhiza echinata L.

Gymnocladus canadensis Lam.

Jacaranda mimosaefolia D. Don.

Kennedya prostrata $\mathrm{R}$. Br.

Laburnum vulgare J. Presl.

Latyrus clymenum L.

- latifolius L.

- Nissolia L.

- odoratus L.

- roseus Stev.

- tingitanus $\mathrm{L}$.

- vernus Bernh.

Lotus ornithopodioides L.

Lourea vespertilionis Desv.

Lupius albus $\mathrm{L}$.

- Barkeri Ldl.

- Cruckshanksii Hook.

- Douglasii Agardh.

- elegans H. B. et K.

- Hartwegii Ldl.

- polyphyllus Ldl.

Medicago intertexta Willd.

- orbicularis All.

Melilotus officinalis Lam.

Mimosa pudica L.

Onobrychis Caput galli Lam.

- crista galli Lam.

Phaseolus multiflorus Willd.

- oblongus Savi.

- vulgaris L.

Rhynchosia phaseoloides DC.

Securigera Coronilla DC.

Soja hispida Moench.

Trifolium elegans Savi.

- pannonicum Jaeq.

Vicia Faba L.

- var. equina $L$. 
- ferruginea Bess.

- narbonensis $\mathrm{L}$.

- villosa Roth.

Vigna glabra Savi.

\section{LInaceae}

Linum campanulatum L.

- flavum L.

- perenne L.

- austriacum L.

- usitatissimum L.

\section{Loasaceae}

Blumenbachia Hieronymi Urb.

Caiophora lateritia Benth.

Loasa tricolor Lindl.

\section{Lobeliaceae}

Lobelia fulgens Willd.

- inflata L.

- syphilitica L.

- triquetra L.

\section{Loganiaceae}

Buddleia Colvilei Hook.

\section{- Lindleyana Fort.}

\section{Lythraceae}

Heimia salicifolia Link et Otto. Lythrum Salicaria L.

\section{Malvaceae}

Abutilon tiliaefolium Sweet.

Althaea rosea Cav.

Anoda hastata Cav.

Gossypium herbaceum L.

Hibiscus Manihot L.

$$
\text { Trionum L. }
$$

Lavatera trimestris L.
Malachra alceaefolia Jacq.

Malope trifida Cav.

Malva crispa $L$.

- silvestris L.

Sida Abutilon L.

\section{Marfynlaceae}

Martynia lutea Lindl.

\section{Melastomataceae}

Bertolonia marmorata Nand.

Centradenia grandifolia Endl.

\section{Moraceae}

Cannabis sativa $\mathrm{L}$.

Ficus edulis Burm.

Humulus Lupulus L.

\section{Myrsinaceae}

Ardisia crispa DC.

\section{Myrtaceae}

Callistemon coccineus F. Muell.

\section{Nolanaceae}

Nolana prostrata L.

\section{Nyctaginaceae}

Mirabilis dichotoma L.

- longiflora L.

Oxybaphus nyetagineus Sweet.

\section{Ochnaceae}

Ochna multiflora DC.

\section{Oenotheraceae}

Clarkia elegans DougI.

- pulchella Pursti.

Epilobium angustifolium $\mathrm{L}$.

- Dodonaei Vill. 
Fuchsia procumbens R. Cunn. Oenothera Drummondii Hook.

- Fraseri Pursh.

- fruticosa L.

- glauca Michx.

- grandiflora Lam.

\section{Oleaceae}

Jasminum odoratissimum L. Ligustrum japonicum Hort. Syringa Emodi Wall.

\section{Orobanchaceae}

Orobanche Hederae Duby.

- lucorum A. Br.

- ramosa $\mathrm{L}$.

\section{Oxalidaceae}

Biophytum sensitivum DC.

\section{Papaveraceae}

Chelidonium majus $\mathbf{L}$.

- var. laciniatum Mill.

Glaucium corniculatum Curt.

- luteum Scop.

Papaver Argemone L.

- bracteatum Lindl.

- dubium L.

- nudicaule L.

- orientale L.

- Rhoeas L.

- somniferum L.

\section{Phytolaccaceae}

Petiveria alliacea $\mathrm{L}$.

Phytolacca decandra L.

Rivina aurantiaca Warsz.

- humilis L.

- laevis L.

\section{Plantaginaceae}

Plantago arenaria Waldst. et Kit.

- Cynops L.

- lanceolata L.

- major L.

- maritima L.

- media L.

- Psyllium L.

- serpentina All.

\section{Plumbaginaceae}

Armeria alpina Willd.

- maritima Willd.

Statice Limonium L.

\section{Polemoniaceae}

Collomia grandiflora Dougl.

Gilia capitata Sims.

- multicaulis Benth.

Phlox Drummondii Hook.

Polemonium coeruleum L.

\section{Polygonaceae}

Fagopyrum tataricum Gaertn.

Polygonum orientale L.

Rheum Emodi Wall.

- officinale Baill.

- palmatum L. var. tanguticum

- undulatum $\mathbf{L}$.

Rumex acetosa L.

- alpinus $\mathrm{L}$.

- patientia L.

- scutatus L.

\section{Porfulacaceae}

Calandrinia compressa Schrad.

Claytonia perfoliata Donn.

Portulaca oleracea L. 


\section{Primulaceae}

Androsace primuloides Duby.

- sarmentosa Wall.

Lysimachia heterophylla Michx.

- vulgaris $\mathrm{L}$.

Primula acaulis Hill. fl. roseo

- Auricula L.

- denticulata Sm.

- kawensis Ht.

- rosea Royle.

- saxatilis Kom.

\section{Ranunculaceae}

Aconitum Anthora L.

- Napellus L.

- ochroleucum Willd.

Anemone Pulsatilla L.

Aquilegia alpina L.

- coerulea James.

- formosa Fisch.

- Skinneri Hook.

- vulgaris $\mathbf{L}$.

Clematis integrifolia $\mathrm{L}$.

Delphinium elatum L.

- formosum Boiss. et Huet.

- grandiflorum L.

- Maackianum Rgl.

- Staphysagria L.

Nigella arvensis $\mathrm{L}$.

- damascena $\mathrm{L}$.

- sativa L.

Paeonia peregrina Mill.

Ranunculus acer L.

- aconitifolius L.

— asiaticus L.

- lanuginosus L.

Thalictrum elatum Jaeq.

Trollius europaeus L.

\section{Resedaceae}

Reseda alba L.

- lutea L.

- luteola L.

\section{Rhamnaceae}

Royle. Ceanothus azureus Desf.

\section{Rosaceae}

Acaena sanguisorbae Vahl.

Cotoneaster horizontalis Decne.

Crataegus pyracantha Medic.

Exochorda Alberti Rgl.

- grandiflora Lindl.

Geum coccineum Sibth. et Sm.

- montanum L.

- rivale $\mathbf{L}$.

Mespilus germanica $\mathrm{L}$.

Neviusia alabamensis A. Gr.

Photinia laevis DC.

Potentilla atrosanguinea Lodd.

- grandiflora L.

- norvegica $L$.

- rupestris $\mathrm{L}$.

- Salesovii Steph.

- verna $L$.

Rhodotypos kerrioides S, et $\mathrm{Z}$. Spiraea Filipendula L.

\section{Rublaceae}

Asperula odorata L.

- tinctoria L.

Galium saccharatum All.

- tricorne Stokes.

Rubia tinctorum L.

\section{Rutaceae}

Citrus trifoliata L.

Dictamnus albus L. 
Erythrochiton brasiliensis Nees. et Mart.

Ruta graveolens L.

\section{Sapindaceae}

Koelreuteria paniculata Laxm.

\section{Saxifragaceae}

Heuchera americana L.

- micrantha Dougl.

- sanguinea Engelm.

Rodgersia aesculifolia Batalin.

- podophylla A. Gray.

Saxifraga Aizoon Jacq.

- crassifolia L.

- Cotyledon L.

- granulata L.

- hypnoides L.

- muscoides Wulf.

- Steinmanni Tausch.

- tricuspidata Rott.

- trifurcata Schrad.

- umbrosa L.

Tiarella cordifolia L.

\section{Scrophulariaceae}

Alonsoa linearis Ruits. et Pav. Antirrhinum majus L.

- Orontium L.

Digitalis ambigua Murr.

- ferruginea $L$.

- lanata Ehrh.

- Intea L.

- purpurea L.

Erinus alpinus L.

Gratiola officinalis L.

Linaria bipartita Willd.

- triphylla Mill.

_- vulgaris Mill.

Maurandia Purpusii Brandegee.
Mimulus cardinalis Dougl.

- luteus L.

- moschatus Dougl.

Nemesia strumosa Benth.

Paulownia imperialis Sieb. et Zucc.

Pentstemon gentianoides Poir.

Rehmannia angulata Hemsl.

Scrophularia alata Gil.

- Neesii Wirtg.

- nodosa $\mathrm{L}$.

- vernalis L.

Torenia Tournieri Lindl.

Verbascum Blattaria L.

- Lychnitis L.

- nigrum L.

- phlomoides L.

- phoeniceum L.

- thapsiforme Schrad.

- Thapsus L.

Veronica gentianoides Vahl.

- urticaefolia L.

\section{Solanaceae}

Atropa belladonna L.

Capsicum annuum $\mathrm{L}$.

Datura laevis L.

- Stramonium L.

- Tatula L.

Hyoscyamus albus L.

- niger $\mathbf{L}$.

Nicandra physaloides Gaertn.

Nicotiana alata Link. et Otto.

- longiflora Cav.

- rustica $\mathrm{L}$.

- Sanderae Ht.

Physalis alkekengi $\mathrm{L}$.

Salpiglossis sinuata Ruitz et Pav.

Solanum Capsicastrum Lk.

- nigrum $\mathrm{L}$. 


\section{Staphyleaceae}

Staphylea colchica Stev.

- pinnata L.

\section{Styracaceae}

Halesia hispida Gard. Chron.

- tetraptera L.

\section{Tropaeolaceae}

Tropaeoleum aduncum Sm.

- majus L.

\section{Ulmaceae}

Celtis occidentalis $\mathrm{L}$.

\section{Umbelliferae}

Aegopodium podagraria L.

Aethusa cynapium L.

Anethum graveolens L.

Anthriscus cerefolium Hoffm.

Angelica officinalis Hoffm.

Astrantia major L.

Carum carvi L.

Chaerophyllum aromaticum L.

- bulbosum L.

Cicuta virosa $\mathrm{L}$.

Conium maculatum $\mathrm{L}$.

Coriandrum sativum $\mathbf{L}$.

Cuminum Cyminum L.

Daucus Carota L.

Eryngium agavifolium Griseb.

- alpinum L.

- amethystinum L.

- eburneum Decne.

- planum L.

Foeniculum vulgare Mill.
Heracleum Lehmannianum Bunge.

- sphondylium L.

Laserpitium Panax Gouan.

Levisticum officinale Koch.

Libanotis montana Cranty.

Myrrhis odorata Scop.

Pastinaca sativa $L$.

Peucedanum officinale $\mathrm{L}$.

Pimpinella magna $\mathrm{L}$.

- rotundifolia Bbrst.

\section{Urticaceae}

Laportea moroides Wedd.

Parietaria officinalis $\mathrm{L}$.

Urtica baccifera L. dioica $\mathrm{L}$.

- pilulifera L.

\section{Valerianaceae}

Centranthus ruber DC.

Valeriana officinalis L.

\section{Verbenaceae}

Clerodendron fallax Lindl.

Verbena officinalis L.

- urticifolia L.

Vitex Agnus-castus L.

\section{Violaceae}

Viola cornuta L.

- tricolor L.

\section{Zygophyllaceae}

Peganum Harmala L.

Tribulus terrestris $\mathrm{L}$.

\section{Prof. Dr. Pefer Stark} Direktor
Rudolf Günther Gartenoberinspektor 
GEDRUCKT BEI

ENGLERT UND SCHLOSSER

FRANKFURT AM MAIN 



\section{Pteridophyta.}

Filicales.

Cyatheaceae.

Alsophila australis R. Br.

Dicksonia antarctica Labill.

\section{Polypodiaceae.}

Asplenium nidus L.

Asplenium Belangerie Kze.

Blechnum brasiliense Desv.

- occidentale L.

Doodia aspera R. Br.

Fadyenia prolifera Hook.

Heminodium crinitum L.

Niphobolus lingua Spring.
Polypodium aureum v. glauca L.

- adnescens S. W.

- Meyenianun Schott.

Schizaeaceae.

Aneimia phyllitidis Sw.

- rotundifolia Schrad.

Osmundaceae.

Osmunda regalis $\mathrm{L}$.

\section{Selaginellaceae.}

Selaginella Emmeliana

- grandis Moore.

Van Geert.

- inaequalifolia Spring.

\section{Gymnospermae.}

\section{Coniferae.}

Cephalotaxus pedunculata Sieb et Zucc.

Taxus baccata $\mathrm{L}$.

- - v. erecta L.

- canadensis Willd.

- parvifolia Wender.

\section{Monocotyledoneae.}

Alismataceae.

Alisma Plantago L.

\section{Araceae.}

Anthurium Barkeri Hook.

Anthurium crystallinum

Arum italicum Mill.

Lind et Arn.

\section{Bromeliaceae.}

Acanthostachys strobilacea Lk. Aechmea bromeliifolia Bak.

- lutea Rgl.
Dyckia remotiflora Otto et Dietr.

- sulphurea C. Koch

Hechtia Ghiesbreghtï Lem.

Pitcairnia undulata Schleich.

\section{Commelinaceae.}

Palisota Barteri Hook.

Tradescantia virginica $L$.

\section{Cyperaceae.}

Carex Grayii Car.

Cyperus alternifolius L. 
- adenophorus Schrad.

- esculentus L.

- Montii L.

- natalensis L.

Scirpus Tabernaemontani Gmel.

— - v. zebrinus

\section{Gramineae.}

Andropogon Sorghum Brot. Avena barbata L.

- fatua L.

- sativa L.

— - v. orientalis Schreb.

-- strigasa Schreb.

Boissiera bromoides Hochst.

Brachypodium distachyum Beauv.

Briza maxima L. media L.

Bromus erectus Huds.

- purpurascens Ait.

Coix Lacryma Jobi. L.

Elymus junceus Fisch.

Festuca gigantea Vill.

, ovina L.

" rubra L.

, virginica $\mathrm{L}$.

Hordeum distichum L.

- hexastichum L.

- maritimum With.

- vulgare L.

- zeocriton L.

Koeleria glauca DC.

Lamarckia aurea Moench.

Melica ciliata L.

Oryza sativa $\mathrm{L}$.

Panicum esculentum A. Br. miliaceum L.

Phalaris canariensis L.

- paradoxa L.

Poa bulbosa L.

Secale cereale L.

- var. perennis L.

Setaria italica Beauv.

- - v. aurantiaca Beauv.

- viridis Beauv.
Sorghum vulgare Pers.

Stipa tortilis Desf.

Triticum dicoccum Schrank.

- durum Desf.

- monococcum L.

- sativum Lam.

— - v. Spelta Hackel.

- vulgare L.

Zea Mays L.

- - v. Caragua

- — , caesia

- - crytosperma Bonaf.

- - , gracillima (L.) Kke.

- _ . Yellow L.

- _ , Naurottola L.

- - , rubra L.

- _. versicolar Bonaf.

\section{Iridaceae.}

Iris aphilla L.

- Chamaeris Bert.

- germanica L.

- juncea Poir.

- japonica Thbg.

- ochroleuca L.

- pumila $\mathrm{L}$.

- plicata Lam.

- spuria L.

- sibirica L.

- tuberosa L.

- umbellata L.

Marica coerulea Ker.

- humilis R. S.

- Northiana Ker.

Sisyrinchium Bermudianum L.

- iridifolium H. B. et K.

\section{Juncaceae.}

Luzula albida D. C.

- Forsteri D. C.

- nemorosa Meyer.

- nivea D. C. 


\section{Liliaceae.}

Agapanthus umbellatus L.'Herit. Allium fallax Don.

,. fistulosum L.

, globosum Bbrst.

., Moly L.

, rotundum L.

Asphodelus fistulosus L.

- tauricus Pall.

Asphodeline liburnica Rchbch. lutea Rchbch.

Bulbine annua Willd.

Chlorophytum amaniense Engl.

- usambariense L.
Danae racemosa $L$.

Eremurus Olgae R. G. M.

- robustus Rgl.

- spectabilis $\mathrm{Rgl}$.

Hypoxis villosa $\mathrm{L}$.

Kniphofia aloides Moench.

- Burchellii Kth.

Majanthemum bifolium D. C.

Veratrum nigrum L.

Zygadenus glaucus Nutt.

\section{Zingiberaceae.}

Globba Schomburgkii Hook.

\section{Dicotyledoneae.}

\section{Acanthaceae.}

Acanthus longifolius Host.

- mollis L.

Aphelandra aurantiaca v.

Roetzlii Lindl.

- squarrosa Leopoldii Rgl.

Daedalacanthus varians VoR.

Thunbergia alata Boj.

\section{Aizoaceae.}

Mesembrianthemum lingui-

forme $L$.

- pyropeum Haw.

Tetragonia echinata Ait.

\section{Amarantaceae.}

Amarantus Blitum L.

- elegantissimus Horn.

- flavus L.

- giganteus Bess.

- retroflexus L.

Celosia cristata L.

,. var. plumosa

\section{Anacardiaceae.}

Rhus vernicifera Dec.
Apocynaceae.

Amsonia angustifolia Mchx.

- Tabernaemontana Wall.

\section{Araliaceae.}

Aralia edulis Zuce.

- japonica Thbg.

- racemosa $L$.

\section{Asclepiadaceae.}

Asclepias syriaca L.

- tuberosa Lin.

Cynanchum nigrum Pers.

Stapelia hirsuta L.

\section{Balsaminaceae.}

Impatiens Balsamina L.

- fulva Nutt.

- Roylei Walp.

- scabrida D. C.

- tricornis L.

\section{Begoniaceae.}

Begonia Schmidtiana Rgl.

- semperflorens L. 


\section{Berberidaceae.}

Berberis Thunbergii D. C. Podophyllum peltatum L.

\section{Bignoniaceae.}

Catalpa Kaempferi D. C. Incarvillea Delavayi B. et Fr.

\section{Borraginaceae.}

Anchusa officinalis L.

Borrago officinlis L.

Cerinthe major L.

Echium Wildpretii H. W. Pearson

Lithospermum officinale L.

Omphalodes amplexicau

Iis Lehm.

- linifolia Moench.

Paracaryum coelestinum LindI.

\section{Calycanthaceae.}

Calycanthus floridus L.

- occidentalis Hook. et Arn.

- praecox L.

\section{Campanulaceae.}

Campanula bonariensis L.

- glomerata L.

- lactiflora M. B.

_ Medium fl. albo. L.

- - fl. coeruleo L.

_ - fl. roseo L.

_ - fl. roseo pl. L.

- persicifolia L.

- rotundifolia Lam.

- Trachelium L.

- turbinata Schott.

- vericolor Sibth. et Sm.

Phyteuma' campanulodes M. B.

Platycodon grandiflorum D. C.

- Mariesii D. C.

Wahlenbergia lobeliodes Desf.

\section{Capparidaceae.}

Polanisia graveolens Rafin.

\section{Caprifoliaceae.}

Lonicera Caprifolium L.

- spinosa Jacquem.

Sambucus racemosa L.

Viburnum Lantana L.

Caryophyllaceae.

Agrostemma Githago L.

- nicaeense Pers.

Dianthus barbatus L.

- deltoides L.

- fragrans Bbrst.

- montanus Heuff.

- neglectus Lois.

- pelviformis L.

- plumarius L.

- superbus L.

Gypsophila elegans Bieb.

- paniculata L.

- repens L.

Lychnis chalcedonica L.

- coronaria Desr.

- Flos Cucili L.

- viscaria Scop.

Melandrium album Garke.

- noctiflorum Fries.

Saponaria officinalis L.

Silene gallica $\mathrm{L}$.

- glauca Zea.

- inflata Sm.

- quinquevulnera L.

- Schafta Gmel.

- viridiflora L.

- Zawadskii Herbich.

Tunica saxifraga Scop.

\section{Celastraceae.}

Evonymus europaeus L.

— latifolius Mill.

\section{Chenopodiaceae.}

Atriplex roseum L.

Beta vulgaris $L$.

Blitum capitatum L.

— virgatum $\mathrm{L}$. 
Chenopodium Quinoa Willd.

Kochia scoparia Schmeiss.

— - v. trichophylla Schmeiss.

Salsola collina Pall.

- Kali L.

- Soda L.

\section{Cistaceae.}

Helianthemum vulgare Gaertn.

\section{Cneoraceae.}

Cneorum triccoccum L.

\section{Compositae.}

Achillae eupatorium v. Parkeri

M. Br.

- Millefolium L.

- nobilis L.

- Ptarmica L.

Ageratum mexicanum Sweet.

Ammobiuum alatum R. Br.

Anacyclus officinarum Heyne

Anaphalis margaritacea Benth.

Arctium Lappa L.

- tomentosum Mill.

Arctotis grandis Thunbg.

Arnica amplexicaulis Wall.

- Chamissonis Less.

- montana L.

Artemisia absinthium L.

- Dracunculus L.

- vulgaris L.

Aster amellus L.

- linosyris Bernh.

- macrophyllus L.

- tibetica Tausch.

Brachycome iberidifolia Benth.

Buphthalmum salicifolium L.

Cacalia suaveolens L.

Calendula officinalis L.

Carlina acanthifolia All.

Catananche coerulea L.

Centaurea atropurpurea W.

- Cyanus L.

- dealbata Willd.
- pulcherrima Willd.

- Rhaponticum L.

- rubrum Der.

Chrysanthemum Balsamita L.

- parthenium Bernh.

- roseum Web. et Mich.

Cirsium heterophyllum All.

Cnicus benedictus Gaertn.

Coreopsis tinctoria Nutt.

Cosmos bipinnatus Cav.

- sulphureus Cav.

Crepis aurea Cass.

- grandiflora Tausch.

- lampsanoides Froel.

- pulchra L.

- ruba L.

- tectorum L.

- sibirica L.

Dimorphotheca pluvialis Mchx. Echinops exaltatus Schrad.

- rito L.

- sphaerocephalus L.

Erigeron bellidifolium Willd.

- speciosus D. C.

Eupatorium aromaticum L.

- cannabinum L.

- Haageanum Rgl. et Kern.

- heterophyllum Dec.

- janthinum Voss.

- purpureum L.

- v. angustifolium L.

Gaillardia aristada Pursh.

Gerbera Anandria Sch.

Gomphrena globosa L.

Helenium autumnale L.

Helianthus annuus L.

Helichrysum bracteatum And.

- monstrosum And.

Hieracium albidum Vill.

- amplexicaule L.

- aurantiacum L.

- bifurcam Bbrst.

- foliosum W. et K.

- Heldreichii Boiss.

- sabaudum Fries.

- umbellatum L. 
- villosum L.

Inula britannica L.

- Helenium L.

- saligna L.

- thapsoides Spr.

Lactuca perennis L.

- virosa L.

Leontopodium alpinum Cass.

Ligularia amplexicaulis D. C.

- macrophylla D. C.

- sibirica Cass.

Matricaria chamomilla L.

Mulgedium macrophyllum D. C.

Onopordon illyricum L.

Parthenium integrifolium L.

Picris echioides L.

Rhagadiolus stellatus Gaertn.

Rudbeckia laciniata L.

- purpurea L.

- triloba L.

Sanvitalia procumbens Lam.

Scorconera hispanica L.

Senecio artemisiaefolius Pers.

- clivorum Maxim.

- Doria L.

- macrophyllus Bieb.

Serratula tinctoria L.

Seriola aethnensis L.

Silphium laciniatum L.

Silybum Marianum Gaertn.

Solidago canadensis L.

- elliptica Ait.

- lanceolata Ait.

- serotina Ait.

- Virgaurea L.

Sonchus Jacquini Dec.

- palustris L.

Spilanthes Acmella L.

- alba L'Herit.

- oleracea L.

Tagetes erecta fl. pl. L.

- patula nana fl. pl. L.

Tanacetum vulgare Bernh.

Telekia speciosa Baumg.

Xanthium spinosum L.

- Strumarium L.
Zacintha verrucosa Gaertn.

Zinnia elegans L.

- Haageana Klotsch.

Convolvulaceae.

Ipomoea tricolor L.

\section{Cornaceae.}

Cornus mas L.

, siberica L.

\section{Crassulaceae.}

Aichryson dichotonum

Web. et Bert.

Anacampseros rufescens D. C.

Bryophyllum crenatum L.

Cotyledon gibbiflora v. metallica

Bak.

Pachyphytum Bak.

Crassula falcata Willd.

Kalanchoë crenata Haw.

. kewensis Haw.

, Kirkii Haw.

rotundifolia Haw.

Kleinia arcticulata Haw.

Sempervivum arboreum L.

- dichotomum D. C.

- glutinosum Ait.

\section{Cruciferae.}

Alyssum saxatile L.

Arabis alpina L.

Barbarea vulgaris $\mathrm{R}$. Br.

Biscutella auriculata L.

Brassica oleracea L.

- nigra Koch.

Bunias Erucago L.

Camelina sativa Crantz.

Cardamine pratensis L.

Cheiranthus Cheiri L.

Capsella bursa pastoris Moench.

Crambe cordifolia Stev.

Draba alpina Lin.

Eruca sativa Mill. 
Erysimum cheiranthoides L.

- Perofiskianum F. v. M.

Hesperis matronalis L.

Hutchinsia brevicaulis Hoppe.

Iberis amara $\mathrm{L}$.

- odorata L.

- pinnata L.

- sempervirens L.

- umbellata L.

Isatis tinctorius L.

Lepidium campestre R. Br.

- ruderale L.

- sativum L.

Lunaria biennis Moench.

- rediviva L.

Myagrum perfoliatum L.

Senebriera Coronopus Poir.

Sinapis alba L.

Sisymbrium officinale Scop.

Vesicaria utriculata Lam.

\section{Cucurbitaceae.}

Bryonia dioica Jacq.

Cyclanthera explodens Naud.

- pedata Naud.

Ecballium Elaterium A. Rich.

Sicyos angulata L.

\section{Datiscaceae.}

Datisca cannabina L.

\section{Dipsacaceae.}

Cephalaria tatarica Schrad.

Dipsacus Fullonum Mill.

Knautia arvensis Couet.

Morina longifolia Wall.

Scabiosa caucasica M. B.

- atropurpurea L.

\section{Elaeagnaceae.}

Elaeagnus longipes A. Gr.

\section{Euphorbiaceae.}

Euphorbia Esula L.

- Gerardiana Jacq.
- Lathyris L.

- Mynsinitis L.

Mercurialis perennnis L.

Ricinus communis L.

\section{Gentianaceae.}

Erythraea Centaurium Pers.

- linariaefolia Auct.

Gentiana acaulis L.

- asclepiadea L.

- cruciata L.

- tibetica Ait.

\section{Geraniaceae.}

Geranium sanguineum L.

- strictum L.

- silvaticum L.

Pelargonium tomentosum Jaeq.

\section{Gesneraceae.}

Achimenes coccineus Benth.

- Durle L.

- hirsuta Benth.

- patens Benth.

Crantzia capitata Hook. Streptocarpus Rexii hybr.

- Wendlandii Rgl.

\section{Globulariaceae.}

Globularia trichosantha F. v. M. - vulgaris L.

\section{Hamamelidaceae.}

Hamamelis virginica $L$.

\section{Hydrophyllaceae.}

Phacelia gongesta Hook.

- tanacetifolia Benth.

\section{Hypericaceae.}

Hypericum calycinum L.

- patulum Thbg.

- perforatum L. 


\section{Labiatae.}

Ajuga reptans $\mathrm{L}$.

Betonica officinalis L.

- stricta Ait.

Calamintha officinalis Moench.

Coleus Rehneltianus L.

- thyreoides Sch.

Dracocephalum peregrinum L.

- secundum R. Br.

Galeobdolon luteum. Huds.

Hyssopus officinalis L.

Lavandula officinalis Cheix.

- vera D. C.

Leonurus cardiaca L.

Leucophaea dasygnaphalia L.

Lophantus anisatus Benth.

Marrubium candidissimum L.

Melissa officinalis L.

Mentha aquatica L.

- piperita L.

- rotundifolia L.

- viridis L.

Monarda clinopodia L.

- didyma L.

- fistulosa L.

Ocimum Basilicum L.

Phlomis tuberosa L.

Physostegia virginiana Benth.

Salvia Aethiopis L.

Salvia candidissima Vahl.

- dumetorum L.

- Horminum L.

- pratensis L.

- Sclarea L.

- splendens Sello.

- sylvestris L.

- verticillata L.

Scutellaria lupulina L.

Stachys germanica L.

- hirsuta H. B.

- liberica Bieb.

Teucrium canadense L.

- Chamaedrys L.

\section{Leguminosae.}

Abrus precatorius L.
Apios tuberosa Mch.

Astragalus Cicer L.

- glycyphyllos L.

Baptisia australis R. Br.

Caragana arborescens Lam.

Cassia floribunda Cav.

Cercis Siliquastrum L.

Cicer arietinum L.

Clianthus Dampieri A. Cunn.

Colutea arborescens $\mathrm{L}$.

- istria Mill.

- orientalis Mill.

Dolichos Catjang L.

- lablab L.

- ornatus Wall.

Galega officinalis L.

Genista sagittalis Lin.

Glycyrrhiza echinata L.

Gymnocladus canadensis Lam.

Indigofera Gerardiana Grah.

Jacaranda mimosaefolia D. Don.

Kennedya rubicunda Vent.

Laburnum vulgare Griseb.

Latyrus Clymenum L.

- latifolius L.

- Iutens L.

- niseolia L.

- odoratus L.

- tingitanus L.

- tuberosus L.

- vernus Berh.

Lotus ornithopodioides L.

Lourea vespertilionis Desv.

Lupinus albus $\mathrm{L}$.

- Barkeri L.

- Cruikshanksii Hook.

- Douglasii Agardh.

- elegans H. B. K.

- Hartwegii L.

- perennis L.

- polyphyllus L.

Medicago intertexta Willd.

Melilotus officinalis Lam.

Mimosa pudica L.

Onobrychis caput galli Lam.

- crista galli Lam. 
Phaseolus atropunctatus Mart.

- capensis L.

- multiflorus L.

- oblongus Savi.

- vulgaris $v$. zebrina L.

Securigera coronilla D. C.

- scorpioides D. C.

Soja hispida Moench.

Trifolium elegans Savi.

- incarnatum L.

- pannonicum Jacq.

Vicia Faba L.

- - v. equina L.

- furruginea Besser.

- tricolor Seb. et Mauri.

- villosa Spr.

Vigna glabra Savi.

\section{Linaceae.}

Linum africanum L.

- alpinum L.

- angustifolium L.

- campanulatum L.

- corymbiferum Desf.

- crepitans Dum.

- flavum L.

- perenne L.

- usitatissimum L.

\section{Loasaceae.}

Blumenbachia Hieronymi Urb. Loasa tricolor LindI.

\section{Lobeliaceae.}

Lobelia cardinalis L.

- fulgens Willd.

- inflata L.

- syphilitica L.

- urens L.

\section{Lythraceae.}

Epilobium angustifolium L.

- Dodonaei Vill.

Heimia salicifolia H. B.
Lythrum hyssopifolium L.

- Salicaria L.

\section{Malvaceae.}

Abutilon tiliifolium Sweet. Anoda hastata Cav.

Althaea rosea Cav.

_... fl. albo Cav.

- fl. purpureo Cav.

_- fl. nigro Cav.

Hibiscus Manihot L.

- Trionum L.

-- syriacus L.

Lavatera trimestris L.

Malachra alceifolia Jacq.

Malope trifida Cav.

Malva crispa L.

- silvestris L.

Sida Abutilon L.

\section{Martyniaceae.}

Martynia lutea Lindl.

- proboscidea hort.

\section{Melastomatacene.}

Bertolonia marmorata Hook.

Centradenia grandifolia Lindl.

\section{Moraceae.}

Cannabis sativa L.

Ficus edulis Burm.

\section{Musaceae.}

Strelitzia Reginae Ait.

Myrsinaceae.

Ardisia crenata Roxb.

\section{Myrtaceae.}

Callistemon coccineus F.v.Muell. Eugenia myrtifolia Sim.

Myrtus communis L. 
Nyctaginaceae.

Mirabilis dichotoma L.

- Jalapa L.

- longiflora L.

Oxybaphus nyctagineus Sweet.

\section{Ochnaceae.}

Ochna multiflora D. C.

\section{Oenotheraceae.}

Fuchsia procumbens R. Cunn.

Oenothera Drummondii Hook.

- Fraseri Pursh.

- fruticosa L.

- glauca Michx.

- grandiflora Lam.

- Lindleyana Dougl.

- muricata L.

\section{Oleaceae.}

Jasminum fruticans L.

Ligustrum japonicum Hort.

Syringa Emodi Wall.

\section{Orobanchaceae.}

Orobanche Hederae Duby.

- lucorum A. Br.

- ramosa L.

\section{Oxalidaceae.}

Biophytum sensitivum D. C.

\section{Papaveraceae.}

Chelidonium majus L.

— - v. laciniata L.

Glaucium corniculatum Curt.

- luteum Scop.

Papaver argemone L.

- bracteatum Lindl.

- dubium L.

- marcrocarpum D. C.

- nuticaule L.

- orientale L.

- paeoniflorum L.

- refractum D. C.

- Rhoeas L.

- somniferum L.

\section{Phytolaccaceae.}

Petiveria alliacea L.

Phytolacca decandra L.

Rivina aurantiaca L.

- humilis L.

- laevis L.

\section{Plantaginaceae.}

Plantago arenaria L.

- Cynops L.

- lanceolata L.

- major L.

- major v. monstrosa L.

- maritima L.

- media L.

- Psyllium L.

- serpentina Lam.

\section{Plumbaginaceae.}

Armeria alpina L.

- maritima Willd.

Statice Limonium L.

\section{Polemoniaceae.}

Collomia grandiflora Dougl.

Gilia capitata Dougl.

- multicaulis Benth.

Plox Drummondii Hook.

- maculata L.

Polemonium coeruleum L.

\section{Polygonaceae.}

Fagopyrum tataricum Gaertn.

Polygonum orientale L.

Rheum Emodi Wall.

- officinale Baill.

- tanguticum L.

- undulatum L.

Rumex Acetosa L.

- alpinus L.

- Patientia L.

- scutatus L.

\section{Portulacaceae.}

Calandrinia compressa Schrad. Portulaca oleracea L. 


\section{Primulaceae.}

Androsace sarmentosa Wall. Lysimachia heterophylla Michx.

- vulgaris L.

Primula acaulis fl. roseo Rogle.

- Auricula L.

- denticulata Sm.

- luteola Rupr.

- rosea L.

\section{Ranunculaceae.}

Aconitum anthora L.

- Napellus L.

- ochroleucum Willd.

- Stoerkianum Rchbch.

- variegatum $\mathrm{L}$.

Anemone Pulsatilla L.

Aquilegia alpina L.

- atrata Koch.

- coerulea James.

- formosa Fisch.

- Skinneri Hook.

- vulgaris L.

Clematis integrifolia L.

Delphinium Ajacis L.

- Consolida L.

- elatum L.

- formosum Boiss.

- grandiflorum L.

- Maackianum Rgl.

- Staphysagria L.

Nigella arvensis L.

- damascena L.

- sativa L.

Paeonia albiflora Pall.

- peregrina Mill.

Pulsatilla vulgaris Mill.

Ranunculus acer L.

- aconitifolius L.

- asiaticus L.

- lanuginosus L.

Thalictrum elatum Jacq.

\section{Resedaceae.}

Reseda alba L.

- lutea L.

- Iuteola L.

\section{Rhamnaceae.}

Ceanothus azureus Desf.

Rhamnus Frangula L.

\section{Rosaceae.}

Acaena sanguisorba Vahl.

Crataegus pyracantha Borkh.

Cotoneaster horizontalis Decne.

Exochorda Alberti Rgl.

- grandiflora Lindl.

Geum coccineum Sibth.

- montanum L.

- rivale $\mathrm{L}$.

Mespilus germanica L.

Neviusia alabamensis A. Gr.

Photinia laevis Dipp.

Potentilla atrosanguinea Lood.

- grandiflora L.

- norvegica $L$.

- Nutalli L.

- Salesovii Steph.

- verna $L$.

Rhodotypus kerrioides S. et Z .

Sorbus aucuparia Gaertn.

Spiraea Filipendula L.

- Froebelli hort.

\section{Rubiaceae.}

Crucianella molluginoides Bbrst.

- aegyptiaca L.

Galium saccharatum All.

- tricorne Hook.

Rubia tinctorium L.

Vaillantia muralis D. C.

Rutaceae.

Citrus trifoliata L.

Dictamnus albus L.

Erythrochiton brasiliensis Nees.

Ruta graveolens L.

\section{Sapindaceae.}

Koelreuteria paniculata Laxm.

\section{Saxifragaceae.}

Heuchera americana L.

- micrantha Dougl.

- sanguinea L. 
Rodgersia aesculifolia Batalin.

— podophylla A. Gr.

Saxifraga Aizoon v. major Jacq.

- crassifolia L.

- Cotyledon L.

- cordata A. Gr.

- granulata L.

- hypnoides L.

- juniperina Adams.

- longifolia Host.

- muscoides Wulf.

- pulchella Don.

- trifurcata Schrad.

- umbrosa L.

Tiarella cordifolia L.

\section{Scrophulariaceae.}

Alonzoa linearis Ruitz. et Pav. Antirrhinum majus

— - fa. Peloria.

Digitalis ambigua Murr.

- ferruginea L.

- geoxiniflora L.

- lanata Ehrh.

- lutea L.

- purpurea L.

- fl. albo

_ fl. albo fa. Peloria

- fl. roseo fa. Peloria

\section{- - fl. roseo}

- purpurea fa. Peloria.

Erinus alpinus L.

Gratiola officinalis L.

Linaria bipartita Willd.

- triphylla Willd.

- vulgaris Mill.

Maurandia Purpursii Brand.

Mimulus cardinalis Dougl.

- luteus L.

Nemesia strumosa Benth.

Paulownia tomentosa C. Koch.

Pentastemon gentianoides Poir.

Rehmannia angulata Hemsl.

Scrophularia alata Gil.

\section{- Neesii Wirtg.}

- nodosa L.

- vernalis $\mathrm{L}$.
Verbascum Blattaria L.

- Lydinitis L.

- nigrum L.

- phlomoides L.

-. phoeniceum L.

- Thapsiforme Schrad.

- Thapsus L.

Veronica gentianoides Vahl.

-_. nrticaefolia L.

\section{Solanaceae.}

Atropa belladonna L.

Capsicum annuum L.

- longum Dec.

Datura laevis Jacq.

- Metel L.

- quercifolium H. B. K.

- Stramonium L.

- Tatula L.

Hyoseyamus albus L.

- niger L.

Nicandra physaloides Gaertn.

Nicotiana alata L.

- longiflora Cav.

- Sanderiana L.

- fl. albo L.

- fl. rubro L.

- rustica.

- virginica Agardh.

Physalis Alkekengi L.

Salpiglossis sinuata Ruitz. et Pav.

Schizanthus grandiflorus hort.

- pinnatus Ruitz. et. Pav.

Solanum laciniatum Ait.

- Lycopersicum L.

- nigrum L.

\section{Staphyleaceae.}

Staphylea colchica Stev.

- pinnata L.

Styracaceae.

Halesia tetraptera L.

\section{Thymelaeaceae.}

Daphne Mezereum L.

Tiliaceae.

Sparmannia palmata E. Méyer 


\section{Tovariaceae.}

Tovaria pendula $R$. et, $P$.

\section{Tropaeolaceae.}

Tropaeoleum aduncum Sm.

- majus L.

\section{Ulmaceae.}

Celtis occidentalis L.

\section{Umbelliferae.}

Aethusa cynapium L.

Ammi majus L.

Anethum graveolens L.

Anthriscus cerefolium Hoffm.

Angelica officinalis Hoffm.

Astrantia Biebersteinii Trautv.

- helleborifolia Salisb.

- major L.

Bifora radiata Bbrst.

Bowlesia tenera Spr.

Bupleurum rotundifolium L.

Carum carvi L.

Chaerophyllum aromaticum L.

- bulbosum L.

Cicuta virosa $\mathrm{L}$.

Conium maculatum $\mathrm{L}$.

Coriandrum sativum L.

Cuminum Cyminum L.

Daucus pulcherrimus Koch.

Eryngium agavifolium L.

- alpinum L.

- amethystinum L.

- bromeliifolium Laroche.

- dilatatum Lam.

- eburneum Desne.

- maritimum L.

- Oliverianum Delar.

- pandanifolium Cham.

- planum L.

- Spinalba Vill.

Foeniculum vulgare Mill.
Heracleum Lehmannianum

- Sphondylium L.

Laserpidium Panax Gouan.

Levisticum officinale Koch.

Libanotis montana All.

Myrrhis odorata Scop.

Pastinaca sativa L.

Peucedanum officinale L.

Pimpinella anisum $\mathrm{L}$.

- magna L.

- rotundifolia Bbrst.

Sanicula europaea L.

Seandix Pecten-Veneris L.

Siler aquilegifolium Gaertn.

Tordylium persicum Boiss.

\section{Urticaceae.}

Laportea moroides Wedd.

Parietaria officinalis $\mathrm{L}$.

Urtica baccifera L.

- Dioica L.

- Dodarti L.

- pilulifera L.

\section{Valerianaceae.}

Centranthus ruber D. C.

Valeriana officinalis L.

\section{Verbenaceae.}

Clerodendron fallax L.

Verbena officinalis L.

- urticifolia L.

Vitex Agnus-castus L.

\section{Violaceae.}

Hymenanthera crassifolia Mast.

Viola tricolor L.

Zygophyllaceae.

Peganum Harmala L.

Tribulus terrestris L.

\section{Prof. Dr. Peter Stark}

Direktor.
Rudolf Günther

Gartenoberinspektor. 



\section{Sämereien}

zum Tausch angeboten

vom

Botanischen Garten der Universität

Frankiturt a. M.

*

Januar 1927.

$\phi$

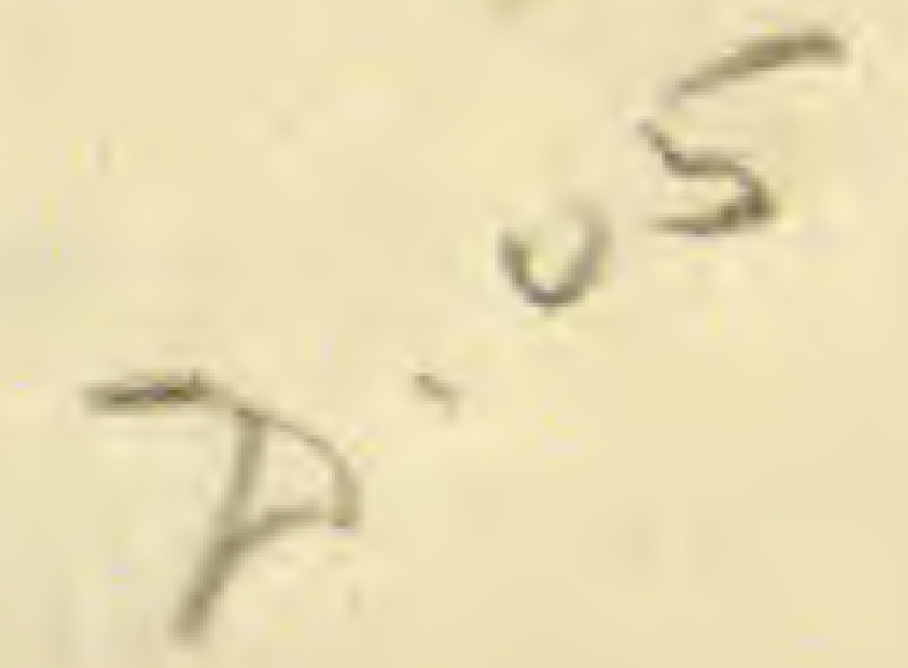




\section{Liliaceae.}

Agapanthus umbellatus L.'Herit. Assphodelus fistulosus Bulbine annua

Willd.

Chlorophytum amaniense Engl. usambariense

Danae racemosa

Eremurus Olgae robustus spectabilis

Kniphofia aloides

" Burchellii

R. G. M.

Rol.

Rol.

Moench.

Kth.

D. C.

Veratrum nigrum

Zygadenus glaucus

\section{Zingiberaceae.}

Alpinia calcarata

Globba Schomburgkii

Roxb.

Hook. Hedychium Gaertnerianum Wall.

Dicotyledoneae.

\section{Acanthaceae.}

Acanthus longifolius

". mollis

Amarantaceae.

Amarantus caudatus

"flavus

". retroflexus

" spinosus

Apocynaceae.

Amsonia angustifolia

Tabernaemontana

Araliaceae.

Aralia japonica

" racemosa

" trifoliata

Asclepiadaceae

Asclepias syriaca

Cynanchum nigrum

\section{Balsaminaceae.}

Impatiens Balsamina

" fulva

" Roylei

Host.

Mchx.

Wall.

Thbg.

L.

L.
Pers.

Nutt.

Walp.
Impatiens scabrida

D. C.

" tricornis

L. M.

Borraginaceae.

Anchusa officinalis

Cerinthe retorta

L.

Echium Wildpetii H.W. Pearson.

Omphalodes linifolia Moench. Paracaryum coelestinum Hock.

Campanulaceae.

Campanula carpatica

lactiflora

M. B.

$\begin{array}{ccc}\text { " } & \text { lactiflora } & \text { M. B. } \\ \text { ". } & \text { Medium fl. albo } & \text { L. } \\ " & \text { fl. coeruleo } & \text { L. } \\ \text { " } & \text { fl. roseo } & \text { L. } \\ \text { " } & \text { fl. roseo pl. Lersicifolia } & \text { L. } \\ \text { " } & \text { L. }\end{array}$

rotundifolia

Schott.

Platycodon grandiflorum A. D. C. Mariesii

Specularia speculum

D. C.

Wahlenbergia lobelioides Desf.

Capparidaceae.

Cleome spinosa

Polanisia graveolens

Rafin.

Rafin.

Caprifoliaceae.

L. Viburnum Lantana

L.

Caryophyllaceae.

Agrostemma Githago

Dianthus barbatus

Pers.

caucasicus

deltoides

fragrans

Bbrst.

montanus

" pelviformis L.

" plumarius

superbus

Gypsophila elegans

Heuff.

L.

" paniculata

Bieb.

Lychnis chalcedonica

L.

L.

" coronaria

" $\quad$ Flos Cuculi

Desr.

L.

" Haageana

Lem.

viscaria

Scop. 
Melandrium album noctiflorum

Saponaria officinalis

Silene aquilegifolia

Gallica
glauca
inflata
$"$ quinquevulnera
$"$ Schafta
".
viridiflora
Zawadskii

Tunica saxifraga

Chenopodiaceae.

Beta vulgaris

Blitum capitatum. virgatum

Kochia scoparia

Schmeiss.

Schmeiss.

\section{Cistaceae.}

Helianthemum vulgare Gaertn.

\section{Cneoraceae.}

Cneorum tricoccum

Compositae.

Achillea eupatorium v. Parkeri
Ptarmica

umbellata

Ageratum mexicanum

Ammobium alatum

Anacyclus officinarum

Artemisia dracunculus

Barkhausia albida

Bidens leucantha

Biotia latifolia

Buphthalmum salicifolium

Cacalia suaveolens

Calendula officinalis

Calliopsis bicolor

Catananche coerulea

Cenia turbinata

Centaurea Cyanus

Chamaepeuce diacantha

Chrysanthemum Balsamita
" rubra

? Parthenium var. eximia
Herbich.

Scop.

Gaert.

Zea.

$\mathrm{Sm}$.

Gmel.

L.

M. Br.

R

Cineraria maritima

Cnicus benedictus

Gaertn.

Colpis rubra

L.

Cosmes bipinnatus Cav.

Crassocephalum aurantiacum $\mathrm{Kz}$.

Crepis grandiflora Tausch.

" lampsanoides Froel.

" pulchra

Dahlia variabilis

Dimorphotheca pluvialis

Echinops exaltatus

Ritro

Erigeron speciosus

Eupatorium aromaticum

cannabinum

" $\quad$ Haageanum Rgl. et Kern. heterophyllum

Helenium autumnale

Helianthus annuus

Mchx.

Schrad.

Dec.

Sweet.

R. Br.

Heyne.

L.

Cass.

Moench.

Willd.

Dec.

L.

L.

L.

Rchbch.

L.

Pers.

D. C.

Silybum Marianum

Tagetes erectus

" grandiflorus

Tanacetum vulgare

Xanthium spinosum

". Strumarium

L.

Bernh.
Paventh.
Hieracium albidum

Helichrysum bracteatum

monstrosum

Heliopsis platyglossa

Inula britannica

" Helenium

, saligna

" thapsoides

Ligularia sibirica

Mulgedium macrophyllum

Parthenium integrifolium

Rhagadiolus stellatus

Rudbeckia laciniata purpurea

Senecio macrophyllum

Seriola aethnensis

Solidago canadensis

$$
\text { " altissima }
$$$$
\text { " elliptica }
$$

Sonhus Jacquini palustris

Spilanthes alba

Vill.

And.

And.

Cass. L.

L.

L.

Spr.

Cass.

D. C.

L.

Gaertn.

M. L.

L.

L. Herit.

L.
Gaertn. 
Zinnia elegans

Haageana

L.

Convolvulaceae.

Klotsch.

Datiscaceae.

,

-

Convolvulus elongatus Wild.

Ipomcea tricolor

Mina lobata

Llav, et Lea.

\section{Crassulaceae}

Cotyledon gibbiflora v. metallica

Crassula falcata

Bak.

Echeveria secunda

Willd.

Sempervivum arboreum

Bak.

\section{Cruciferae.}

Alyssum saxatile

Arabis alpestris v. ciliata

Arabis alpina

Barbarea vulgaris

Biscutella auriculata

Brassica oleracea

Bunias Erucago

Camelina sativa

Cheiranthus Cheiri

Capsella bursa pastoris Moench.

Crambe cordifolia

Draba alpina

Eruca sativa

Erysimum cheiranthoides

\section{Perofskianum \\ " repandum}

Hesperis matronalis

Iberis amara

" pinnata

" sempervirens

, umbellata

Lunaria biennis

Myagrum perfoliatum

Rhagadiolus stellatus

Sinapis alba

Sisymbrium alliaria

\section{Cucurbitaceae.}

Cyclanthera explodens

$$
\text { pedata }
$$

Ecballium Elaterium

Sicyos angulata
Stev.

Lin.

R. Br.

Mill.

F. v. M.

L.

L.

L.

L.

L.

Moench

L.

Gaern.

L.

Scop.

Naud.

Naud.

A. Rich.

L.
Datisca cannabina

L.

Dipsacacea.

Cephalaria tatarica

Schrad.

Scabiosa caucasica

M. B.

Euphorbiaceae.

Euphorbia Lathyris

Myrsinitis

Mercurialis perennis

Ricinus communis

L.

L.

L.

L.

Gentianaceae.

Erythraea centaurium

Gentiana acaulis

$\begin{array}{ll}\text { " } & \text { cruciata } \\ " & \text { macrophyllum } \\ " & \text { Olivieri } \\ \text { " } & \text { tibetica }\end{array}$

Geraniaceae.

Erodium gruinum

Geranium ibericum

Pers.

L.

L.

Griseb.

Ait.

" sanguineum

Willd.

Cav.

L.

L.

Pelargonium tomentosum Jacq.

Gesneraceae.

Streptocarpus Rexii hybr.

"Wendlandii

Rgl.

Halorrhagidaceae.

Gunnera chilensis

Hippuris vulgaris

Lam.

Hydrophyllaceae.

Nemophila maculata

Phacelia congesta

Benth.

$\begin{array}{ll}\text { grandiflora } \\ \text { malvifolia } \\ \text { ", } & \text { tanacetifolia } \\ \text { viscida }\end{array}$

Hook.

Labiatae.

Ajuga reptans

A. Gr.

Chamiss.

Benth.

Torr.
Betonica officinalis

L.

Ait.

Calamintha officinalis Moench.

Coleus Rehneltianus

thyrsoides

Sch.

Dracocephalum secundum R. Br. 
Lavandula officinalis

Leonurus cardiaca

Lophanthus anisatus

Mentha aquatica

" rotundifolia

Monarda clinopodia

" didyma

Nepeta camphorata

" cyanea

, grandiflora

Ocimum Basilicum

Phlomis tuberosa

Salvia dumetorum

Horminum

" pratensis

" $"$ fl. rubro

" Sclarea

" sylvestris

" splendens

". verticillata

Stachys germanica

\section{Leguminosae.}

Abrus precatorius

Astragalus glycyphyllos

Baptisia australis

Caesalpinia Sappan

Caragana arborescens

Cassia floribunda

Carmichaelia australis

Cicer arietinum

Clitoria ternata

Colutea istria

orientalis

Cytisus Alschingeri

Dolichos Catjang

$$
\text { lablab }
$$

Galega officinalis

Genista tinctoria

Glycyrrhiza echinata

Indigofera australis

Kennedya rubicunda

Latyrus Clymenum

$$
\text { Nissolia }
$$

odoratus

tingitanus

tuberosus
Cheix.

L.

Benth.

L.

L.

L.

L.

L.

L.

Boiss.

Stev.

M. B.

L.

L.

L.

L.

L.

L.

L.

Sello.

L.

L.

L.

L.

R. Br.

Lin.

Lam.

Cav.

R. Br.

L.

Mill.

Mill.

Vent

L.

L.

L.

L.

Willd.

Vent.

L.
L.
L.
L.
L.
Lotus ornithopodioides

Lupinus albus

angustifolius

Barkeri

Cruikshanksii

Douglasii

elegans

Hartwegi

perenne

polyphyllus

Medicago intertexta

Melilotus officinalis

Mimosa pudica

Onobrychis caput galli

L.

Hook.

Agardh.

H. B. K.

crista galli

Phaseolus atropunctatus

capensis

multiflorus

Mungo

oblongus

radicans

tuberosus

L.

L.

L.

Willd.

Lam.

Lam.

Lam.

Mart.

vulgaris v. zebrina

Securigera coronilla scorpioides

Soja hispida

L.

L.

Savi.

Lour.

L.

D. C.

D. C.

Moench.

Tordylium persicum

Tetragonolobus purpureus

Moench.

Trifolium fragiferum

incarnatum

montanum

pannonicum

procumbens

Vicia cassubica

" Faba

" $\quad$ v. equina

ferruginea

villosa

Vigna glabra

L.
L.
L.
L.
L.
L.
L.
L.
ser.
Spr.
avi.

Linaceae.

Linum alpinum

austriacum

corymbosum

flavum

grandiflorum

perenne

usitatissimum 
Loasaceae.

Blumenbachia Hieronymi Urb. Cajophora lateritia Loasa tricolor

Benth. Lindl.

\section{Lobeliaceae.}

Lobelia cardinalis

$$
\begin{array}{ll}
" & \text { fulgens } \\
\text { inflata } \\
\text { ". } & \text { syphilitica } \\
& \text { Lythraceae. }
\end{array}
$$

Epilobium angustifolium Lythrum hyssopifolium Salicaria

\section{Malvaceae.}

Abutilon tiliifolium

Althaea rosea

Sweet. $\begin{array}{lll} & \text { fl. albo Cav. } \\ \text { " } & \text { fl. purpureo Cav. } \\ \text { " fl. nigro Cav. }\end{array}$

Hibiscus Trionum syriacus

Malachra alceifolia Jacq. Malope trifida Cav. Malva crispa

Pavonia spinifex Sparmannia palmata E. Meyer. Melastomaceae.

Bertolonia marmorata " primuliflora

Sonerila margaritacea

Hook.

Hook.

Lindl.

Myrsinaceae.

Ardisia crenata

Roxb.

Nyctaginaceae.

Oxybaphus nyctagineus

Ochnaceae.

Ochna multiflora

D. C.

\section{Oenotheraceae.}

Clarkia elegans pulchella

Godetia amoena

Oenothera grandiflora muricata

" purpurea

Sweet.

Dougl.

Pursh.

Lam.

L.

Curt.
Orobanchaceae.

Orobanche Hederae

Duby. " lucorum

A. Br.

\section{Papaveraceae.}

Chelidonium majus

L.

v. laciniata L.

Slaucium corniculatum Curt.

Papaver argemone

" bracteatum

" dubium

" macrocarpum

" orientale

" paeoniflorum

" refractum

". Rhoeas

" somniferum

Scop.

Lindl.

Phytolaccaceae.

Phytolacca decandra

Rivina aurantiaca

" humilis

" laevis

D. C.

Plantaginaceae.

Plantago arenaria

D. C.

L.

$\begin{array}{lll}" & \text { Cynops } & \text { L. } \\ " & \text { major v, monstrosa } & \text { L. } \\ " & \text { maritima } & \text { L. } \\ " & \text { Psyllium } & \text { L. }\end{array}$

Plumbaginaceae.

Armeria alpina

"I maritima

Willd.

Statice Limonium

Polemoniaceae.

Gilia capitata

" multicaulis

Phlox Drummondii

Dougl.

Benth.

Hook.

\section{Polygonaceae.}

Atraphaxis lanceolata

Emex spinosa

D. C.

Fagopyrum tataricum

Campd.

G. Dorn, Polygonum monspeliense Desf.
Rheum Emodi

officinale

undulatum
Wall.

Baill. 
Rumex alpinus

". patientia

, scutatus

Portulacaceae.

Calandrinia compressa Schrad. " sericea Hook, et Arn.

\section{Primulaceae.}

Androsace sarmentosa

Primula cortusoides

denticulata

" lutea

" mollis

" verticillata

Samolus Valerandi

Ranunculaceae.

Aconitum anthora

Stoerkianum

variegatum

Aquilegia coerulea

Skinneri

Cimicifuga racemosa

Delphinium Consolida

$$
\text { elatum }
$$

Myosurus minimus

Nigella arvensis

" damascena

"sativa

Paeonia albiflora

Pulsatilla Halleri vulgaris

Thalictrum silvaticum

Resedaceae.

Reseda alba

$$
\text { " r luteola }
$$

Rhamnaceae.

Ceanothus azureus

\section{Rosaceae.}

Acaena sanguisorba Crataegus pyracantha Geum montanum

$$
\begin{array}{ll}
\text { " } & \text { reptans } \\
\text { strictum } & \text { Rossii }
\end{array}
$$

I.

Rchbch.

L.

James.

Hook.

Nutt.

L.

L.

L.

L.

Pall.

Willd.

Mill.

Koch.

Desf.

Vahl.

Borkh.

L.

L.

Ait.

Ser.
L. Potentilla Salesowii

L. Rhodotypus kerrioides

L. Sanguisorba canadensis

Rubiaceae.

Crucianella aegyptiaca

Galium saccharatum tricorne

Wall. L. $\mathrm{Sm}$.

I,am. Hook. Forsk. L.

Vaillantia hispida

\section{Rutaceae.}

Dictamnus albus

Ruta graveolens

L.

Saxifragaceae.

Heuchera micrantha

sanguinea

Saxifraga juniperifolia

trifurcata

" umbrosa

Tiarella cordifolia

Scrophulariaceae.

Antirrhinum majus

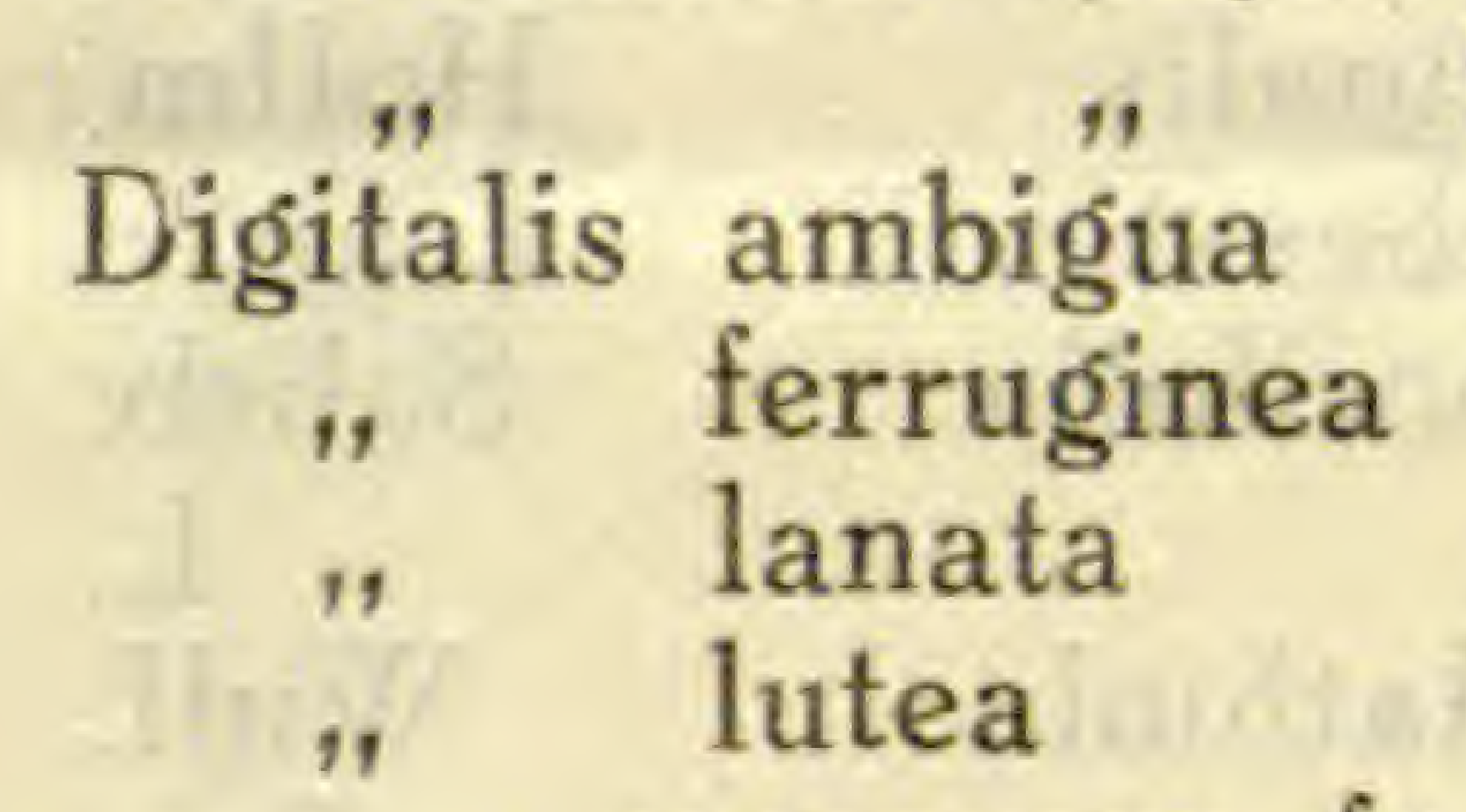

fa. Peloria.

Adans.

Schrad.

Dougl.

L.

L.

L.

" purpurea fa. Peloria.

Erinus alpinus

Linaria bipartita

triphylla

L. Maurandia Purpusii

L. " luteus

L. Scrophularia alata

Murr.

" nodosa

Verbascum Blattaria

nigrum

phoeniceum

Veronica pectinata

Ehrh.

L. 
Datura laevis Stramonium

Jacq.

Tatula

Hyoscyamus albus

$$
\text { " niger }
$$

Nicandra physaloides

Nicotiana alata

$$
\text { " } \quad \text { longiflora }
$$

Salpiglossis sinuata Ruitz. et $\mathrm{Pav}$

Solanum lycopersicum

$$
\begin{array}{ll}
" & \text { nigrum } \\
" & \text { pyranthum } \\
& \text { Tropaeolaceae. }
\end{array}
$$

Tropaeolum aduncum

$$
\text { " majus }
$$

\section{Ulmaceae.}

Celtis occidentalis

$$
\text { Umbelliferae. }
$$

Ammi majus

Anethum graveoléns

Anthriscus cerefolium

Angelica officinalis

Astrantia Biebersteinii helleborifolia

Athamanta Mathiola

Bowlesia tenera

Bupleurum rotundifolium Chaerophyllum aromanticum

$$
\text { " aureum }
$$

Lin.

L.

L.

L.

Gaert.

L.

Cav.

L.
L.

L.

Lam.

Sm.

L.

L.

L.

Hoffm. Hoffm, Trautv. Salisb.

Wulf.

Spr.

L.

L.
Coriandrum sativum

Eryngium bromelifolium Laroche,

$$
\begin{array}{ll}
\text { " } & \text { dilatatum } \\
\text { eburneum } \\
\text { " Spinalba }
\end{array}
$$

Lam.

Desne.

Foeniculum vulgare

Ferula asa-foedita

Ferulago

Heracleum Sphondylium Levisticum officinale Malabaila Hacquetii

Myrrhis odorata

Pastinaca sativa

Peucedanum officinale

Sanicula europaea

Scandix Pecten $=$ Veneris

Siler aquilegifolium

Vill.

Mill.

Urticaceae.

Laportea moroides

Urtica baccifera

" pilulifera

Koch.

Tausch

Scop.

L.

L.

L.

L.

Gaertn.

Valerianaceae.

Centranthus ruber

Valeriana Phu.

Wedd,

L.

L.

\section{Violaceae.}

Hymenanthera crassifolia Mart. Viola cornuta

", tricolor

L.

Zygophyllaceae.

Tribulus terrestris

L.

\section{Prof. Dr. M. Möbius}

Direktor.

\section{Rudolf Günther}

Garteninspektor. 




\section{ae 343}

\section{SÄMEREIEN}

zum Tausch angeboten

vom

\section{Botanischen Garten in Frankfurt am Main. 1912.}

\section{Pteridophyta.}

\section{Filices,}

Adiantum Bausei L.

- caudatum L.

- crenatum Fisch.

- formosum R. Br.

- gracillimum v. Houtte,

Alsophila australis $\mathrm{Br}$.

Aneimia Phyllitidis Sw.

- rotundifolia Sw.

Aspidium aculeatum Schwartz.

- angulare Kit.

- coriaceum Sm.

- Filix mas var. monstrosum Schwartz,

- spinulosum Schwartz.

- violaceum Lk.

Asplenium Belangeri Kze.

- bulbiferum Forst.

- furcatum Sm.

- Nidus avis L.

- septentrionale Hoffm.

Athyrium alpestre Nyl.

- australe Prsl.

Blechnum brasiliense Desf.

- giganteum Hort.

Davallia dissecta L.

Dicksonia antarctica Labill.

Doodya media R. Br.

Gymnogramme ehrysophylla Kze.

Hemionitis palmata L.

Lygodium japonicum Sw.

Nephrolepis acuminata Kuhn.

- acuta Schk.

- Bausei Hort.

- exaltata Schott.
Onoclea Struthiopteris Hoffm.

Ophioglossum vulgatum L.

Osmunda regalis $\mathrm{L}$.

Platycerium alcicorne Desv.

- grande Sm.

- Hillii Moore.

Polypodium aureum v. glauca L.

Pteris aquilina L.

- ensiformis Burm.

- gracilis Dec.

- longifolia L.

- marginata B.

- palmata Willd.

- pedata L.

- serrulata var. cristata Veitch

Scolopendrium daedaleum L.

Todea barbara Moore.

Woodwardia angustifolia Sm.

- caudata Cav.

- radicans Sm.

\section{Selaginellaceae.}

Selaginella cuspidata Link.

- Emmeliana spring.

- flagellifera Bull.

- Martensii Spring.

\section{Coniferae.}

Callitris verrueosa $\mathrm{R} . \mathrm{Br}$. $\hbar$ Sequoia sempervirens Endl. $\hbar$ Taxus baccata L. $\hbar$ 
Monocotyledoneae.

\section{Alismaceae.}

Alisma Plantago L. 이

Sagittaria chinensis Sims. 2/

- montevidensis Schlecht. 24

- sagittifolia L. 2

\section{Amaryllidaceae.}

Amaryllis nivea Schult. 24

Cooperia Drummondi Hook. 2

Hippeastrum aulicum Herb of

Zephyranthes atamasco L. 가

\section{Araceae.}

Anthurium magnificum Lind. 2) - Scherzerianum Schott. 24 Arum italicum Mill. of

Sauromatum guttatum Schott. 2

Zantedeschia aethiopica Spreng. 24

\section{Bromeliaceae.}

Aechmea Drakeana Rgl. 94 Billbergia longifolia Lindl. 24

- violacea Bert. 9

- zebrina Lindl. 9

Caraguata lingulata Lindl. 9)

Dyckia remotiflora Otto Dietr. 2 - sulphurea Lem. ?

Encholirion corallinum Mart. 2)

Pitcairnia punicea Scheidw. 24

- spathacea Mey. 24

\section{Butomaceae.}

Butomus umbellatus L. 24

\section{Cannaceae.}

Canna indica L. 29

\section{Commelynaceae.}

Commelyna coelestis Willd. 2

Tinantia erecta Schlecht. 24 Tradescantia pilosa Lehm. 24
Cyperaceae.

Carex glauca Scop. 이

- japonica fol. var. L. 24

- leporina L. 2

- maxima Scop. 21

- pendula Huds. 2

- sylvatica Huds. 21

- xanthophysa Wahlbg. 2

Cladium Mariscus R. Br. 24

Cyperus laxus Thbg. 24.

- longus L 2).

Isolepis prolifera $\mathrm{R}$. $\mathrm{Br}$. 2)

Scirpus Holoschoenus Link. 2)

- lacustris L. 24

- var. zebrina L. 2

- maritimus L. 24

- radicans Schkuhr. 24

- sylvaticus L. 와

\section{Dioscoreaceae.}

Dioscol ea japonica Thbg. 24 - verticillata Lam. 2)

Tamus communis L. 24

\section{Gramineae.}

Alopecurus arundinaceus Poir. 2 Andropogon Sorghum Brot. $\odot$

Avena barbata Roth. 2

- fatua L. $\odot$

- sativa Schreb. $\odot$

- sterilis L. $\odot$

- strigosa Schreb. $\odot$

Briza geniculata Thbg. $\odot$

- maxima L. ㅇ․

- media L. ९f

Bromus arvensis L. $\odot$

- asper L. 2

- erectus Huds. 9

- macrostachys Desf. $\odot$

- maximus Desf. $\odot$

- mexicanus Desf. $\odot$

- secalinus L. $\odot$

Calamagrostis Epigaios L. थ

Chloris barbata Sw. $\odot$

Coix Lacryma L. $\odot$

Corynephorus canescens Beauv. 24

Dactylis glomerata L. $\mathcal{4}$

Desmazeria sicula Dum. $\odot$

Elymus arenarius L. 9

- giganteus Vahl. 24

- glaucifolius Muhlbg. 2

Festuca gigantea Vill. ?

- glauca Lam. 24

- ovina L. 4 
Festuca rubra L. 와 - sylvatica Vill. 아

Hierochloa borealis R. S. 2 4

Hordeum distichum L. $\odot$

v. zeocriton L. $\odot$

- hexastichon L.

- $\quad$ v. pyramidata Kke. $\odot$

- maritimum With. $\odot$

- vulgare L. $\odot$

- $\quad$ v. coelestis L. $\odot$

Koeleria cristata Pers. $\hbar$

Lasiagrostis Calamagrostis Link 2

Melica altissima L. 24

- ciliata L. 2

- exasperata R. S. 2

Molinia coerulea Moench. 24

Nardurus unilateralis Boiss. $\odot$

Panicum capillare L. $\odot$

- miliaceum L. $\odot$

- $\quad$ - var. alba L. $\odot$

- $\quad-\quad$ nigra Willd. $\odot$

- virgatum L. 2

Pennisetum compressum R. Br. - Rüppellii Steud. $\odot$

Phalaris canariensis L. $\odot$

- minor Retz. $\odot$

- paradoxa L. $\odot$

Phleum asperum Vill. $\odot$

Poa alpina L. 94

- bulbosa var. vivipara L. 2

Stipa capillata L. 2

Triticum ciliatum Dec. $\odot$

- monococcum L. $\odot$

- polonicum L. $\odot$

- $\quad$ - var. villosa. L $\odot$

- sativum compactum Hackel var. cretica. $\odot$

- $\quad$ - dicoccum Hackel. $\odot$

- $\quad$ - Spelta Hackel. $\odot$

var. alba. $\odot$

var. Duhaleniana. $\odot$

Zea Mais L. $\odot$

- - var. caesia. $\odot$

- - - Caragua Gmel. $\odot$

- - - cryptosperma Bonaf. $\odot$

- - Jellow L. $\odot$

- - - Naurottolo L. $\odot$

- - - orizoides Kke. $\odot$

- - - rubra L. $\odot$

- - - tuscana L. $\odot$

_ _ - xanthomis Kke.

\section{Iridaceae.}

Anomatheca cruenta Lindl. 44 Iris lutescens Lam. 2/

- mauritanica Spr. 2
Iris sibirica L. 2

Sisyrinchium anceps Lam. 24 .

- Bermudianum L. 2

\section{Juncaceae.}

Juncus glaucus Sibth. 2|

- squarrosus L. 9

Luzula albida Desv. 9

- multiflora Desv. 2

\section{Juncaginaceae.}

Triglochin maritimus L. 2

\section{Liliaceae.}

Agapanthus praecox Willd. 2

- umbellatus L'Her. 24

- - var. albiflora Voss. 2

Albuca fastigiata Dryand. ㅇ․

Allium cernuum Roth. 24

- fallax Schult. 24

- fistulosum L. 2f

- fragrans Vent. 24

- globosum M. B. 2

- Moly L. 24

- moschatum L. 2

- nigrum L. 9

- odorum L. 9

- Porrum L. 24

- rotundum L. 2

- strictum Schrad. 2

- sphaerocephalum L. 24

- ursinum L. 24

Aloe abyssinica Lam. 2

- glabra Salm. 2y

Anthericum Liliago L. ?

Asparagus officinalis L. 2

- Sprengeri Rgl. थ

Asphodelus albus Willd. 2

- fistulosus L. 24

Asphodeline liburnica Rchb. \&

- lutea L. 24

Bowiea volubilis Haworth. 2

Bulbine annua Willd. 24

Eremurus robustus Rgl. 와

Eucomis regia Ait. 와

- stricta Ait. 24.

Fritillaria imperialis L. 9

- Meleagris L. 4

Funkia ovata Spr. 2

- Sieboldiana Lodd. 2

Galtonia candicans Desne. 24

Haworthia granata Haw. 24

Hemerocallis flava L. 와 
Kniphofia Tuckii Bak. 2

- uvaria Hook. 9

Lilium Martagon L. 이

Muscari comosum Willd. 2

- racemosum Mill. 24

Nothoscordum fragrans Kth. 9

Ornithogalum narbonense L. भI

Polygonatum giganteum Otto et Dietr.2

- 1atifolium Desf.

- - var. commutata Schult. 2

- multiflorum All. 2

- officinale All. 2

- verticillatum Schult. 24

Ruscus Hypophyllum L. 2

- racemosus L. 2

Scilla hispanica Mill. 의

- undulata Desf. 24

Smilax aspera L. 24

Smilacina racemosa Desf. 2

- stellata Desf. भI

Tulipa Didieri Jord. ?।

- Kolpakowskyana Rg1. 24

Uropetalum serotinum Ker. ㅇ

Urginea maritima Bak. 24

Veratrum album L. 2)

- nigrum L. ?

Zygadenus bracteatus Schult. 2

- glaucus Nutt. 2

\section{Typhaceae.}

Sparganium simplex Smth. 24

Typha angustifolia L. 24

- latifolia L. 24

- minima Funk. 24

\section{Dicotyledoneae.}

\section{Acanthaceae.}

Acanthus longifolius Poir. 2

- mollis L. 24

- niger Mill. 2

Ruellia Devosiana hort. 2

- strepens L. 24

\section{Aizoaceae.}

Mesembrianthemum barbatum L. 2

- crystallinum L. $\odot$

- glaucum L. $\hbar$

- pinnatifidum L. $\odot$

Tetragonia crystallina L'Hérit. $\odot$

\section{Amarantaceae.}

Amarantus albus L. $\odot$

- caudatus L. $\odot$

- - fl. rubro L. $\odot$

- - fl. flavo L. $\odot$

- hypochondriacus L. $\odot$

- paniculatus L. $\odot$

- speciosus L. $\odot$

- - v. monstrosa L. $\odot$

Celosia cristata L. $\odot$

- - var, aurantiaca L. $\odot$

- - plumosa L. $\odot$

- - - splendens L. $\odot$

\section{Anacardiaceae.}

Rhus vernicifera Dec. 4

\section{Apocynaceae.}

Amsonia angustifolia Michx. 2

- latifolia Michx. 24

- Tabernaemontana Walt. 24

- salicifolia Pursh. ?f

Apocynum cannabinum K. 아

\section{Araliaceae.}

Acantopanax sessiliflorum Seem. $\hbar$

\section{Aristolochiaceae.}

Asarum canadense L. $\mathscr{4}$

- europaeum L. 2

\section{Asclepiadaceae.}

Asclepias fruticosa L. 아

- speciosa Torr. 24

- syriaca L. 24

- verticillata L. 9|

- virgata Balb. 2।

Cynanchum nigrum Moench. 24

- Vincetoxicum L. 2

\section{Balsaminaceae.}

Impatiens Balsamine L. $\odot$

- cristata W. $\odot$

- fulva Nutt. $\odot$

- glanduligera Royl. $\odot$

- longicornis Wall. $\odot$

- Roylei Walp. $\odot$ 
Begoniaceae.

Begonia gracilis Jacq. ?f

\section{Bignoniaceae.}

Incarvillea Delavayi Franch. 2 - Olgae Rgl. 24

\section{Borraginaceae.}

Anchusa italica Retz. 24

Borrago officinalis L. $\odot$

Caccinia strigosa Boiss. 9

Cerinthe retorta $\mathrm{Sib}$. $\odot$

Echinospermum macranthum Ledeb.-) Myosotis palustris L. 24

Omphalodes linifolia Moench. $\odot$

Paracaryum coelestinum Voss. -

Symphytum asperrimum Sm. 이

- bohemicum Schmidt 24

- officinale L. 과

\section{Calycanthaceae.}

Calycanthus occidentalis Hook. $\hbar$ Chimonanthus fragrans Lindl. $\hbar$

\section{Campanulaceae.}

Campanula alliariifolia Willd. $\mathscr{4}$

- 1atifolia Fisch. 2

- Medium var alba L. $\odot$

- - - - plena L. $\odot$

- - - calycantha L. $\odot$

- - - coerulea L. (-)

- - - - rosea Llena $\mathrm{L}, \odot$

- - - - plena L. $\odot$

- nobilis Lindl. 2

- persicifolia var coerulea L. 24

- - L. 2

- rapunculoides L. 2

- rotundifolia L. ข|

- strigosa Vahl. $\odot$

Phyteuma Scheuchzeri All. 24

Platycodon autumnale Desne. 아

- grandiflorum DC. 2

Specularia falcata Dec. $\odot$

- Speculum DC. $\odot$

\section{Cannabinaceae.}

Cannabis sativa $\mathrm{L}$. $\odot$

Humulus Lupulus L. 2)

\section{Capparidaceae.}

Cleome spinosa Jacq. $\odot$

Polanisia graveolens Rafin. $\odot$

\section{Caryophyllaceae.}

Agrostemma Githago L., $\odot$

- nicaeensis Willd. $\odot$

Cucubalus baccifer Lin. 2f

Dianthus barbatus I. 2

- deltoides L. 2

- plumarius L. 2

- sylvestris Wulf. 21

Drypis spinosa L. $\odot$

Gypsophila altissima L. 9

- cerastioides Don. 29

- elegans Bbrst $\odot$

- paniculata L. 24

- perfoliata L. भ

- repens L. 2

Herniaria glabra L. 2

Lychnis chalcedonica L. 2

- diurna Sibth. (-)

- Flos Jovis Lam. 2y

- Flos Cuculi L. 29

Melandrium album Garke. 2

- rubrum Garke. 24

Saponaria elegans Lapeyr. 2

- officinalis L. 아

- Vaccaria L. 2

Silene alpestris Jacq. 24

- Armeria L. $\odot$

- Drummondi Hook. 2

- glauca Zea. $\odot$

- Hornemanni Steud 2

- muscipula L. $\odot$

- paradoxa L. 9

- pendula L. $\odot$

- quinquevulnera L. $\odot$

- viridiflora L. 24

- Zawadskyi Presl. 24

Tunica prolifera Scop. 2

- saxifraga Srop. 아

Viscaria alpina Fries. 2

- Satorii Boiss. 24

- viscosa Aschers. 2

\section{Chenopodiaceae.}

Atriplex hortensis L. $\odot$

- laciniata L. $\odot$

- rosea L. $\odot$

Basella ramosa Jacq. $\odot$

Blitum virgatum L. $\odot$

Chenopodium album L. $\odot$

- Bonus Henricus cymigera L. $\odot$ 
Kochia scoparia Schrad. $\odot$ Salsola crassa Bib. $\odot$ - Kali L. $\odot$

\section{Cistaceae.}

Cistus populifolius L. $\hbar$

- salicifolius L. $\hbar$

- salvifolius L. $\hbar$

Helianthemum grandiflorum DC.

- umbellatum Mill. $\hbar$

- variabile Sw. 2

\section{Compositae.}

Achillea ageratifolia v. Airon Bth. 2

- cartilaginea Ledeb. 4

- lanata Spr. 24

- Millefolium L. 4

- nobilis L. 9

- Ptarmica L. 2

- - fl. pl. L. 2

Alfredia cernua Cass 2

Anthemis austriaca Jacq. 4

- tinctoria L 4

Arnica montana Lin. 9

Artemisia Absinthium L. 24

- Dracunculus L. 2f

Aster alpinus L. 29

- amellus L. 24

- himalayensis Hook. 2

- Linosyris Bernh. 2|

Bidens leucantha Willd. $\odot$

Cacalia suaveolens L. भf

Calais Bigelowii A. Gray. $\odot$

Calendula officinalis L. $\odot$

Carlina acaulis L. 24

Carthamus tinctorius L. $\odot$

Cenia turbinata Pers. $\odot$

Centaurea alpina L. 이

- calocephala Willd. 94

- Cyanus L. 8

- imperialis alba L. $\odot$

- macrocephala Pusk. 2

- melitensis L. $\odot$

- pulcherrima Willd. 4

- Scabiosa L. 2

- strobilacea Scop. $\odot$

Chrysanthemum Balsamita L. 24

- carinatum Schrad. $\odot$

- corymbosum L. ?

- Leucanthemum L. 2

- roseum Web, et Moor. 24

- vulgare Bernh. 24

Cirsium canum All. 9

- heterophyllum All. 2

Cnicus benedictus L. $\odot$
Coreopsis cardaminifolia Torr. et Gr. $\odot$

- longipes Hook. 2

- palmata Nutt. 24

- tinctoria Nutt. $\odot$

Cosmos sulphureus Cav. $\odot$

Crepis latifolia Ball. 의

- longifolia Ball. 2

- pulchra L. 24

- rubra L. $\odot$

- sibirica L. 2

Cynara Cardunculus L. $(-)$

Dahlia coccinea Cav. 아

- variabilis Desf. 24

Dimorphotheca pluvialis Moench. $\odot$ Doronicum marcrophyllum Fisch 4

- Pardalianches L. 4

Echenais carolinoides Cass. :-

Echinops dahuricus Fisch. 와

- globifer Frank. 24

- humilis Bieb. 24

- sphaerocephalus L. 24

Endoptera Dioscoridis Dec. $\odot$

Erigeron speciosus Dec. 가

Eupatorium aromaticum L. 2

- cannabinum L. 2

- Haageanum Regel. et Kern. 2

- heterophyllum Dec. 2

- grandiflorum Dec. 2

- purpureum L. 24

- Purpusii Brand. 24

Gaillardia aristata Pursh. 24

- pulchella Foug. $\odot$

Gnaphalium purpureum L. 2

Grindelia arguta Schrad. 4

- integrifolia Dec. 24

Haplopappus croceus A. G. 2 .

Hedypnois persica Bbrst. $\odot$

Helianthus annuus L. $\odot$

Helminthia echioides Gaert. $\odot$

Hieracium alpinum L. 24

- aurantiacum L. 2

- amplexicaule L. 21

- dentatum Hoppe. 2

- eriophorum Link. 24

- Lactaris Bert. 24

- lycopsifolium Froel. 2y

- pulmonarioides Vill. 24

- ramosum Suter. 2

- sabaudum L. 2

- sylvestre Tausch. 4

- villosum Link. ?

Humea elegans Sm. (-) Hypochaeris glabra L. $\odot$

Inula germanica L. ?

- glandulosa Willd. 2

- Helenium L. 9

- hirta L. 24

Inula media Bbrst. 2)

- saligna L. 4 
Inula thapsoides L. 2

Kentrophyllum lanatum Dec. $\odot$

Lactuca perennis L. 2

- saligna L. $\odot$

- Scariola L. 24

- virosa L. 2

Lagascea mollis Cav. $\odot$

Lampsana communis L. $\odot$

Leontopodium alpinum Karst. 2

- himalayanum Dec. 2

Ligularia marcrophylla Dec. 2

- sibirica Cass. 24

- speciosa F. M. 2

- thyrsoidea Dec. ?

Madaria elegans Dec. $\odot$

Madia sativa Molin. $\odot$

Maruta Cotula Bbrst. $\odot$

Matricaria Chamomilla L. $\odot$

Mulgedium macrophyllum Dec. 아

- palustre L. 2

- sibiricum Less. 2

Onopordon Acanthium L. $\odot$

- elongatum L. $\hbar$

Palafoxia texana Hook.

Parthenium integrifolium L. 24

Picris hieracioides L. 2)

Prenanthes purpurea L. 2)

Pulicaria dysenterica Gaert. 2

- odora Rchbch. ?

Pyrethrum corymbosum Willd. 9

- macrophyllum W. 24

- roseum Bbrst. 2|

Rhagadiolus caudatus L. $\odot$

- stellatus Gaert. $\odot$

Rhaponticum scariosum Lam. ㅇ

Rudbeckia digitata Mill. भf

- columnaris Pursh 2

- laciniata L. 24

- speciosa Wender. 24

Santolina Chamaecyparis $\mathrm{L} \hbar$ - rosmarinifolia L. $\hbar$

Sanvitalia procumbens Lam. $\odot$

Scolymus maculatus L. $\odot$

Scorzonera villosa Scop. 2

Senecio artemisiifolius Pers. 2

- Doria L. 2

- Jakobaea L. $\odot$

- marcrophyllus M. B. 24

- palustris L. $\odot$

Serratula coronata L. 9

- gigantea L. 2

- heterophylla Desf. 24

- radiata Bbrst. 24

- tinctoria L. 24

Silphium integrifolium Mchx. 우

- laciniatum L. F

- perfoliatum L. 24

- trifoliatum L. 24

Silybum Marianum Gaertn. $\odot$
Solidago canadensis L. ?

- lanceolata Ait. 24

- serotina Ait. 21

- verrucosa Schrad. 21

Spilanthes oleracea L. $\odot$

Tragopogon porrifolius $\mathrm{L} \odot$

Verbesina enceliodes B. Hgp. $\odot$

Xanthium macrocarpum Dec. 24

- spinosum L. $\odot$

- Strumarium L. $\odot$

Xeranthemum cylindricum Smith. $\odot$

Zacyntha verrucosa Gaert. $\odot$

Zinnia verticillata Andr. $\odot$

\section{Convolvulaceae.}

Convolvulus arvensis L. 요

- pentapetaloides L. $\odot$

- pseudosiculus Cav. $\odot$

- tricolor L. $\odot$

Cuscuta europaea L. $\odot$

Ipomaea coccinea L. $\odot$

- Nil Rth. $\odot$

- Purga Wender. 24

- purpurea Lam. $\odot$

\section{Coriariaceae.}

Coriaria myrtifolia L. $\hbar$

\section{Cornaceae.}

Benthamia fragifera Lindl. $\hbar$

\section{Crassulaceae.}

Bryophyllum calycinum Salisb. 의 - crenatum Backer 24

Crassula falcata Ait. 24

Sempervivum tabuliforme Haw. 아

Umbilicus oppositifolius DC. 9

- pendulinus DC. 9

- Pestalotzii DC. 구

\section{Cruciferae.}

Aethionema grandiflorum Boiss, et Hohen. 24

- pulchellum Boiss. et Hohen 4 Alyssum argenteum All. 24

Arabis albida Stev. 24

- hirsuta Scop. $\odot$

Berteroa incana Dec.

Biscutella apula L. $\odot$

- auriculata L. $\odot$

- eriocarpa Dec. $\odot$ 
Brassica nigra Koch. $:-$

- oleracea L. ()

Bunias Erucago L. $\odot$

- orientalis L. $\odot$

Cakile maritima Scop. $\odot$

Camelina microcarpa Andriz. $\odot$

- sativa Crantz. $\odot$

Cardamine pratensis L. ㅇ

Cheiranthus Cheiri L. $\odot$

Cochlearia danica L. $\odot$

Crambe cordifolia Stev. 2

- grandiflora Dez. 4

- hispanica L. $\odot$

- orientalis L. 21

Draba contorta Ehrh.थ

Erysimum perfoliatum Crantz. $\odot$

- Perofskianum Fisch. et May. $\odot$

Farsetia clypeata R. Br. $\odot$

Hesperis matronalis L. 24

Hymenophysa pubescens Mayer. 4

Iberis amara L. $\odot$

- Garrexiana All. $\hbar$

- pinnata L. $\odot$

- saxatilis L. 2

- umbellata L. $\odot$

Isatis tinctoria L. $\odot$

Jonopsidium acaule Rchb. $\odot$

Lepidium crassifolium W. K, $ף$

- latifolium L. 9

- sativum L. $\odot$

Lunaria annua L. $\odot$

Malcolmia chia Dez. $\odot$

- maritima R. Br. $\odot$

Moricandia hesperidiflora Dez. $\odot$

Myagrum perfoliatum L. $\odot$

Nasturtium amphibium R. Br. 4

Raphanus caudatus L. $\odot$

Ricotia Lunaria DC. $\odot$

Senebiera Coronopus Poir. $\odot$

Sinapis alba L. ()

Sisymbium Irio. L. $\odot$

- officinale Scop. $\odot$

- strictissimum L. 아

- tanacetifolium L. 24

Zilla myagroides Forsk. 나

\section{Cucurbitaceae.}

Bryonia alba L. 아

- dioica Jacq. 와

Citrullus Colocynthis Schrad. $\odot$

Cyclanthera explodens Naud. $\odot$

Ecballium Elaterium L. $\odot$

Lagenaria claviformis hort. $\odot$

- maxima Ser. $\odot$

- vulgaris v. Gurda Ser. $\odot$

- - v. pyrotheca Ser.

- - v. sphaerica hort. $\odot$

Sicyos angularis L. $\odot$

\section{Datiscaceae.}

Datisca cannabina L. 24

\section{Dipsacacae.}

Cephalaria ambrosioides R. S. 24

- centaureoides R. S. 24

- tatarica R. S. 2

Dipsacus fullonum Mill. $\odot$

- laciniatus L. $\odot$

- sylvestris Mill.

Morina longifolia Wall. 2)

Scabiosa altropurpurea Desf. (

- caucasica Bbrst. 2

- palaestina L. $\odot$

- prolifera L. $\odot$

- suaveolens Desf. 21

\section{Ericaceae.}

Lyonia calyculata Rchbch. $\hbar$

\section{Euphorbiaceae.}

Euphorbia cyathophora Jacq. (-)

- Esula L. 24

- Gerardiana Jacq. 2

- globosa Sims. 24

- Lathyris L. 2f.

- Myrsinitis L. 21

- nicaeensis All 2f

- orientalis L. 24

- trigonocarpa Lodd. 2

- verrucosa L. 2

Mercurialis annua L. $\odot$

Phyllanthus juglandifolius Willd. $\hbar$

Ricinus communis L. $\odot$

\section{Frankeniaceae.}

Frankenia ericifolia C Smith. 24

\section{Gentianaceae.}

Erythraea Centaurium Willd. (-) Gentiana Olivieri Griceb. 24

- punctata L. 24

- tibetica King. 24

- verna L. 24

Limnanthemum geminatum Griseb. 24 Menyanthes trifoliata L 24

Swertia perennis L 24 


\section{Geraniaceae.}

Erodium gruinum Willd. $\odot$

Geranium aconitifolium L. 2

- canescens Herit. 24

- cristatum Stev. 24

- ibericum Cav. 2

- palustre L. 24

- pratense fl. albo L. 2

- sanguineum L. 2

- sylvaticum L. 2

Pelargonium coronopifolium Jacq.

- tomentosum Jacq. $\hbar$

\section{Gesneriaceae.}

Ramondia Myconi F. Schulz. 2

Streptocarpus caulescens Hook. 4

- kewensis Hook. 24

- Veitchii hort. 2

- Wendlandii L. 2

\section{Globulariaceae.}

Globularia bellidifolia Ten. 24

- cordifolia C. 24

- vulgaris L. 2

\section{Halorrhagidaceae.}

Gunnera chilensis Lam, 24

Halorrhagis alata Jacq. $\hbar$

Hippuris vulgaris L. 2

\section{Hydrophyllaceae.}

Nemophila insignis Doug1. $\odot$ Phacelia tanacetifolia Benth. $\odot$ - viscida Torr. $\odot$

Wigandia caracasana H. B. et Kth.

\section{Hypericaceae.}

Hypericum calycinum L. $\hbar$

$$
\begin{aligned}
& \text { - grandiflorum Salisb. } 24 \\
& \text { = perforatum L. भ| } \\
& \text { - pulchellum L. भ| }
\end{aligned}
$$

\section{Labiatae.}

Calamintha Nepeta Link. 24

Dracocephalum grandiflorum L.

- peregrinum L. 9

Elscholtzia cristata Willd. $\odot$
Hyssopus officinalis L. 24

- - fl. albo L. 24

Lamium album L. 2

- garganicum L. 4

- Orvala L. 2

Lavandula vera $\mathrm{DC}$ ?

Leonurus capitatus L. 2

- Cardiaca Adans. 24

- sibiricus L. $\odot$

Lophantus anisatus Benth $\hbar$

Lycopus exaltatus L. 24

Marrubium vulgare L. 24

Melissa Acinos Benth. $\odot$

- officinalis L. 4

Mentha crispa Alef. 2

- piperita L. 2

- Pulegium L. 24

- sylvestris L. 2

Monarda didyma L. 24

- fistulosa L. 4

- mollis L. 24

Molucella spinosa L. $\odot$

Nepeta cyanea Steven. 4

- grandiflora M. B. 24

- latifolia DC. 24

- tuberosa L. 2

Origanum vulgare L. 와

Perilla nankinensis Voss. $\odot$

Phlomis tuberosa L. 24

Physostegia virginica Benth. 2

Plectranthus fruticosus. L'Herit. 2

Prunella grandiflora L. 2

- vulgaris L. ?4

Salvia argentea L. $\odot$;

- dumetorum Andr. 2

- glutinosa L. 2

- officinalis L. 2

- pratensis L. 2

- - v. abnormis L. 4

- $\quad$ v. fl. rubra 2

- Sclarea L. 2

- sylvestris L. 2

- verticillata L. 2

- $\quad$ - fl. albo L. 24

Scutellaria alpina L. 24

- Lupulina L. 2

Sideritis montana L. ㄱ.

Stachys circinnata L.'Herit. 24

- germanica L. 9

- lanata Jacq. 24

- longifolia Benth. 24

- palustris L. 24

- recta L. 2y

- setifera C. A. Meyer. 2

- sylvatica L. 2

Teucrium Botrys L. $\odot$

- Chamaedrys L. $\odot$

- Scorodonia L. 2

Thymus vulgaris L 2 . 
Lauraceae.

Persea carolinensis Nees. $\hbar$

\section{Leguminosae.}

Astragalus boeticus L. $\odot$

- Cicer L. 2

- galegiformis L. 2

- ponticus Pall. 24

- stipulatus Don. 2

- utriger Pall. 4

Baptisia australis R. Br. 아

Callistachys lanceolata Vent. $\hbar$

Caragana Altagana Poir. $\hbar$

- arborescens Lam. $\hbar$

Cicer arietinum L. $\odot$

Coronilla scorpioides Dec. $\odot$

Cytisus Linkii Janka. $\hbar$ - vulgaris Griseb. $\hbar$

Desmodium canadense DC. $\hbar$

Dolichos Catjang L. $\odot$

Dorycnium latifolium Willd. $\hbar$

Galega officinalis L. 2

- orientalis Lam. 24

Genista elatior Koch. 24

- radiata Scop. 9

- scoparia Lam. 24

- thyrsiflora Booth. $\hbar$

- tinctoria L. $\hbar$

- $\quad$ - var. virgata $\mathrm{W} . \hbar$

Glycyrrhiza echinata L. 24 foetida Desf.

- lepidota Pursh. 7

Hedysarum grandiflorum Pall. 2

- obscurum L. 4

- sibiricum Poir. 24

Indigofera argentea $\mathrm{L} \hbar$

- Dosua Hamilt. $\hbar$

- pulchella hort. $\hbar$

- tinctoria L. $\hbar$

Lathyrus Aphaca L.

- articulatus L. $\odot$

- Clymenum L. $\odot$

- heterophyllos L. 24

- latifolius L. 29

- maritimus L. $\odot$

- mexicanus Wender. $\odot$

- niger Bern. 4

- Nissolia L. $\odot$

- Ochrus Dec. $\odot$

- odoratus L. $\odot$

- pseudaphaca L. $\odot$

- tuberosus L. 2

- vernus Bernh. 24

Lespedezia bicolor Turez. $\hbar$

Lotus biflorus Ser. $\odot$

- ornithopodioides L. $\odot$

- Tetragonolobus L. $\odot$
Lotus Tetragonolobus v. biflora Ser. $\odot$

- uliginosus L. 2f

Lupinus Barkeri Lindl. $\odot$

- Cruikshanksii Hook. $\odot$

- Douglasii Agardh. $\odot$

- Hartwegii Lindl. $\odot$

- perennis L. 9

- polyphyllus Lindl. 2

Medicago falcata L. 와

- glutinosa Bbrst. 2/

- marginata Willd. $\odot$

- sativa L. 2

- scutellata Lam.

Melilotus albus Desr. $\odot$

- coeruleus Desr. $\odot$

- macrorrhiza Pers. 2

- officinalis Pers. (-)

Onobrychis crista galli Lam $\odot$

- Caput galli Lam. $\odot$

- sativa Lam. 4

Ononis spinosa L. 2

- Natrix L. 2

Orobus laevigatus W. K. 2

- luteus L. 4

- niger L. भ|

- pallescens Bbrst. 2

- vernus $\mathrm{L}$.

Phaseolus capensis L. $\odot$

- compressus Dec. $\odot$

- multiflorus Lam. $\odot$

- radiatus L. $\odot$

- tuberosus Lour. 4

- vulgaris L. $\odot$

- - var. Zebrina L. $\odot$

Pocockia cretica Dec. $\odot$

Securigera Coronilla Dec. $\odot$

Soja hispida Moench. $\odot$

Sophora flavescens Ait. 2y

Spartium junceum Link. $\hbar$

Swainsonia galegifolia $\mathrm{R}$. $\mathrm{Br}$. $\hbar$

Trifolium alpestre L. Of

- elegans Sav. 4

- fragiferum L. 21

- incarnatum L. 24

- pannonicum Jacq. 4

- procumbens L. $\odot$

- rubens L. 2

Trigonella calliceras Fisch. $\odot$

- corniculata L. $\odot$

- Foenum graecum L. $\odot$

Vicia angustifolia Roth. $\odot$

- cassubica L. 4

- Cracca L. 24

- dumetorum L. 9

- Faba L. $\odot$

- ferruginea Besser. $\odot$

- Orobus Dec. 24

- sativa L. $\odot$

Vigna glabra Savi. $\odot$ 
Linaceae.

Linum alpinum L. 24

- angustifolium Huds. 24

- aquilinum Dec. $\odot$

- austriacum L. 2

- corymbiferum Desf. 24

- flavum L. 24

- grandiflorum Desf.

- Lewisii Pursh. थ

- perenne L. 24

- usitatissimum L. $\odot$

\section{Loasaceae.}

Blumenbachia Hieronymi Urb. $\odot$ Cajophora lateritia Presl. $\odot$ Loasa papaverifolia H. B. Kth. $\odot$ - tricolor Lindl.

- triphylla Juss. $\odot$

Mentzelia oligosperma Nutt. $\odot$

\section{Lobeliaceae.}

Lobelia syphilitica L. 24 - urens L. $\odot$

\section{Loganiaceae.}

Buddleia curviflora et Arn. Hook. $\hbar$ -- japonica Hemsl. $\hbar$

\section{Lythraceae.}

Cuphea Llavea Llav. et Lex. $\mathcal{H}$ - petiolata L. 2)

Lythrum lineare L. 24

- Salicaria L. 2

\section{Malvaceae.}

Abutilon tiliaefolium Fisch. $\odot$ Althaea cannabina L. 2 f

- officinalis L. 4

- rosea Cav. 9

- - fl. albo Cav. $\odot$

- - v. monstrosa Cav. $\odot$

- - fl. purpureo Cav. $\odot$

- - fl. nigro Cav..

- taurinensis Dec. 24

Anoda hastata Cav. $\odot$

Hibiscus esculentus L. $\odot$ - syriacus $\mathrm{L}$. $\hbar$

- Trionum L. $\odot$

Kitaibelia vitifolia Willd. $\odot$
Lavatera trimestris L. $\odot$

Malachra alceifolia Jacq. $\odot$

Malope malacodes L. $\odot$

- trifida Cav. $\odot$

Malva caroliniana crispa L. $\odot$

- crispa L. $\odot$

- nicaensis All. $\odot$

\section{Myrtaceae.}

Baeckea virgata Andr. $\hbar$

Callistemon pungens G. Don. $\hbar$ - rugulosum Dec. $\hbar$

Leptospermum myrtifolium Sieb. $\hbar$ Melaleuca cuticularis Labil. $\hbar$

- hypericifolia Smith. $\hbar$ Myrtus communis L. $\hbar$

Tristania conferta R. Br. $\hbar$

- laurina R. Br. $\hbar$

\section{Nolanaceae.}

Nolana prostrata L. $\odot$

\section{Nyctaginaceae.}

Mirabilis divaricata L. $\odot$

- Jalapa L. $\odot$

- longiflora L. $\odot$

- viscosa Cav. $\odot$

Oxybaphus nyctaginea Swet 4

\section{Nymphaeaceae.}

Nuphar luteum L. 2

\section{Ochnaceae.}

Ochna multiflora Dec. $\hbar$

\section{Onagraceae.}

Clarkia elegans Dougl. $\odot$ Epilobium Dodonaei Vill. 24 Jussiaea Blumeana Dec. 4 - salicifolia H. B. 2 Lopezia coronata Andr. $\odot$ Oenothera amoena Lchm. $\odot$

- Fraseri Pursh. 24

- fruticosa L. 24

- glauca Michx. 24

- grandiflora Lam. (-)

- odorata Jacq. 24

- pusilla Michx. 24

- purpurea Curt. $\odot$ 


\section{Orobanchaceae.}

Orobanche Hederae Duby. 24

- ramosa L. 2

- speciosa D. C. 2

\section{Oxalidaceae.}

Biophytum proliferum DC. 2

- sensitivum DC. 24

Oxalis corniculata L. var, fol, atropurpureis L. $\odot$

- stricta L. 2

- valdiviensis Barn.

\section{Papaveraceae.}

Argemone mexicana L. $\odot$

Chelidonium majus L. थ

Corydalis ophiocarpa Hook. et Thoms. 2

Eschscholtzia californica Chmss. (-)

Glaucium corniculatum v, tricolor Curt. $\odot$

- luteum Scop. 9

Macleya cordata R. Br. 24

Meconopsis cambrica Vign. 인

Papaver alpinum L. 2

- bracteatum Lindi. 2

- caucasicum Bbrst. थ

- nudicaule L. 2

- orientale L. 24

- Rhoeas L. $\odot$

- somniferum L. $\odot$

\section{Passifloraceae.}

Passiflora coerulea L. 24

- gracilis Link. 2

\section{Pedaliaceae.}

Martynia fragrans Lindl. $\odot$

- proboscidea Glox. $\odot$

- lutea L. $\odot$

\section{Phytolaccaceae.}

Phytolacca decandra L. थ - Kaempheri A. Gray. 24 Rivina aurantiaca Wood. 2 - humilis L. $\hbar$

\section{Plantaginaceae.}

Plantago alpina L. 2

- arenaria W. K. 4

- gentianoides Sm. 4

- Lagopus L. 4

- lanceolata L. 24

- major L. 4

- major var. purpurascens L. $\mathscr{Y}$

- maritima L. 24

- Psyllium L. $\odot$

\section{Plumbaginaceae.}

Armeria Welwitschii Boiss, 아 Goniolimon tataricus Boiss. 2 Statice virgata Willd. थ

\section{Polemoniaceae.}

Cobea scandens Cav. 24

Collomia grandiflora Dougl. $\odot$

Gilia capitata Dougl. $\odot$

- tricolor Benth.

Phlox decussata L. 2)

- rosea hort. 24

- suaveolens Ait. 2

Polemonium coeruleum L. $\mathcal{4}$

- pauciflorum Watz. 2

\section{Polygonaceae.}

Atraphaxis lanceolata DC. $\odot$

Polygonum orientale L. $\odot$

- sachalinense F. Schmidt. 2

- virginianum L. 24

- viviparum L. 2

Rheum Emodi Wall. 24

- Rhaponticum L. 29

- undulatum L. 2

Rumex Acetosa L. 24

- crispus L. 24

- Patientia L. 94

- purpureus Poir. 94

- salicifolius Weinm. 24

\section{Portulacaceae.}

Calandrinia Menziesii Hook. $\odot$

- sericea Hook. 24

- umbellata DC. $\odot$

Portulaca oleracea L. $\odot$

Telephium Imperati Lin. 2f

Telinum patens Willd. $\odot$ 


\section{Primulaceae.}

Androsace elongata L. $\odot$

- maxima L. $\odot$

- septentrionalis L. $\odot$.

Lysimachia ciliata Lin. 2)

- punctata Lin. of

- quadrifolia L. 2

- thyrsiflora L. 24

- verticillata Bbrst. 2

Primula Auricula L. 24

- japonica A. Gray. 2

- officinalis Jacq. 4

\section{Ranunculaceae.}

Aconitum Anthora L. 2)

- Lycoctonum L. 29

- Napellus L. 24

- septentrionale Mart. 2

Actaea palmata Dec. 24 - spicata L. 24.

Adonis aestivalis $\mathrm{L}$.

Anemone alpina L. 2)

- fulgens Gay. 24

- multifida Poir. 2

- narcissiflora L. 2

- palmata L. ㅇ

- sylvestris L. 2

- virginiana L. 2

Aquilegia coerulea James. 4

- glandulosa Fisch. 24

- Skinneri Hook. 24

- vulgaris L. ?|

Caltha palustris L. 2/

Cimicifuga racemosa Nutt. 9

- serpentaria Pursh. 24

Clematis angustifolia Jacq. 24

- gebleriana Bong. 4

- integrifolia L. 24

- flammula L. 2

- Viorna L. $\hbar$

- viticella L. 24

Delphinium Ajacis L. $\odot$

- cashmerianum Royle. 가

- cheilanthum Fisch. 24

- elatum L. 24

- exaltatum Ait. 2

- grandiflorum L. 21

- intermedium Ait. 24

- Maackianum Kgl. 2

- montanum L. 24

- palmatifidum Dec. 9

Nigella damascena L. $\odot$

- orientalis L. $\odot$

- sativa L. $\odot$

Paeonia corallina Retz. 24

- officinalis L. 2

- $\quad$ - fl. pl. Pall. 4
Paeonia sinensis Pall. 24

- $\quad$ var. rosea hort. 2

Pulsatilla Halleri Willd. ?

Ranunculus cassubicus L. 2

- muricatus L. $\odot$

- repens L. 94

- serbicus Vis. 2

Thalictrum aquilegifolium L. 24

- cinereum Desf. 24

- Cornuti L. 2

- elatum Jacq. 9

- foetidum L. 24

- glaucum Desf. 2

- minus L. 2

- purpurascens L. 24

- sylvaticum Koch. 24

- tuberosum L. 24

Trollius altaicus C. A. Mey. 2

- asiaticus L. 2

- europaeus L. 24

\section{Resedaceae.}

Astrocarpus sesamoides DC. $\odot$ Reseda abyssinica Fresen. $\odot$

- alba L. $\odot$

- glauca L. 2

- luteola L. $\odot$

- myriophylla Tenor. 24

- odorata L. $\odot$

\section{Rhamnaceae.}

Pomaderris elliptica Labil. $\hbar$

\section{Rosaceae.}

Acaena glauca hort. 24

- ovalifolia R. P. 24

- sanguisorba Vahl. 2

Agrimonia dahurica Willd.

- Eupatorium L. 94

- odorata Mill. 2

- leucantha Kunze. 24

- repens L. 2

- suaveolens Pursh. 2

Alchemilla fissa Günth. 2)

- vulgaris L. 24

- Dryas octopetala L. 24

- lanata Kern. 24

Geum carolinianum Walt. 2

- coccineum Sm. 24

- japonicum Thbg. 2

- ranunculoides Serring. 24

- rivale L. 2

- Rossii Ser.

- strictum Ait. 24 
Mespilus germanica $L$. $\hbar$ Potentilla alpestris Hall. 2

- atrosanguinea Don. 24

- collina Wibel. भ

- inclinata Vill. 24

- palustris L. 9

- rupestris L. 2

Rhodotypus kerriodes S. et Z. $\hbar$

Rosa gallica L. $\hbar$.

- laxa Retz. $\hbar$

- pimpinellifolia L. $\hbar$

- turbinata Ait. $\hbar$

Sanguisorba canadensis L. 와

- mauritanica Desf. 24

- officinalis L. 9)

- tenuifolia Fisch. 24

Sibbaldia procumbens L. 2

Spiraea lobata hort. of

\section{Rubiaceae.}

Asperula arvensis L. 2)

- galioides M. B. 24

- humifusa Bess. 2f

- odorata L. 24.

- tinctoria L. 24

Cruciannella aegyptiaca L. $\odot$

- angustifolia L. $\odot$

- gilanica Trin. 24

Galium boreale L. 2f

- Mollugo L. 29

- purpureum L.

- tricorne With. $\odot$

Rubia tinctorium L. 2)

Sherardia arvensis L. 와

Vaillantia hispida L.

\section{Rutaceae.}

Dictamnus albus L. 24

- albus var. ruber L 2

Melicope ternata Forst $\hbar$

Ptelea trifoliata L. $\hbar$

Ruta graveolens L. 24

\section{Sapindaceae.}

Koelreuteria paniculata Laxm. $\hbar$

\section{Saxifragaceae.}

Francoa appendiculata Cav, 24 Heuchera alba A. Gray. 24

- americana L. 9

- cylindracea Lindl. 2

- hispida Pursh. 24
Heuchera micrantha Doug1. 24

- sanguinea A. Gray. ข|

Parnassia palustris. L. ㅇ

Saxifraga Aizoon L. 와

- caespitosa L. ?|

- cartilaginea Willd 24

- ceratophophylla Ait. 24

- cordifolia L. 24

- Cotyledon L. 2

- erassifolia L. 9

- Cymbalaria L. 2

- decipiens L. 24

- granulata L. 2|

- longifola Lapeyr. 2|

- rodundifolia L. 24

- tenella Wulf. 91

- umbrosa L. 24

- - fol. var. L. 24

Tiarella cordifolia L. थ

\section{Scrophulariaceae.}

Anthirrhinum majus L. If

- - var peloria L. 24

- Orontium L. $\odot$

Calceolaria scabiosifolia Sms. $\odot$

Collinsia bicolor Benth $\odot$

Digitalis ambigua Murr. y

- ferruginea L $\odot$

- gloxiniiflora L. $\odot$

- - var. pura L. $\odot$

- Ianata Ehrh. 9/

- lutea L. 21

- purpurea L. 2

- - var. alba L. 24

- - var. rosea L. 24

- viridiflora Lindl. 2 .

Erinus alpinus L. ?

Gratiola officinalis Lin 2

Linaria heterophylla Desf.

- lusitanica Spr. 24

- triphylla Willd.

Mauraridia scandens Gray. (

Mimulus cardinalis Lin. $\odot$

- hybridus hort. 24

- luteus L. 24

Pentastemon barbatus Nutt. 24

- confertus Doug1. 24

- diffusus Dougl. 24

- gentianoides Don. 9

- glaber Pursh. 2f

- glaucus Grah. 24

- hirsutus Willd. 24

- laevigatus Ait. भf

Scrophularia nodosa L. 9

- vernalis L. 2

Torenia exapendiculata Rg1. $\odot$

- parviflora Wall. 24

Verbascum Blattaria L. $\odot$ 
Verbascum Kanitzianum Link. (-

- Lychnitis L. $\odot$

- nigrum L. 24

- orientale Bbrst. (-)

- phlomoides L. $\odot$.

- phoeniceum L. 4

- thapsiforme Schrad. $\odot$

- Thapsus L. 24

Veronica gentianoides Vahl. 24

- incana Vahl. 24

- officinalis L. 24

- orchidea Crantz. 24

- polystachya Link. 24

- spicata L. 2f

- spuria L. 24

- urticifolia L. 24

- virginica L. 24

- $\quad$ - var. sibirica L. 24

\section{Solanaceae.}

Atropa Belladonna L. 24

Capsicum cerasiforme hort. $\odot$

Datura ferox L. $\odot$

- Metel L. $\odot$

- Strammonium L. $\odot$

- Tatula L. $\odot$

Hyoscyamus albus L. $\odot$

- niger L. $\odot$

Lycopersicum cerasiforme var. rubra Don. $\odot$

- piriforme var. rubra Voss. $\odot$

Mandragora officinalis Bertol. 24

Nicandra physaloides Gaert. $\odot$

Nicotiana affinis Moore. $\odot$

- Langsdorffi Schrad. $\odot$

- rustica L. $\odot$

- Tabacum L. $\odot$

Nierembergia gracilis Hook. $\odot$

Petunia nyctaginiflora Juss. $\odot$

Scopolia lurida Link et Otto. 와

Solanum diphyllum L. $\hbar$

- Gilo Raddi. $\odot$

- hederodoxum Dun. $\odot$

- marginatum L. $\hbar$

- miniatum Bernh.

- Pseudo-Capsicum L. $\hbar$

Thymelaeaceae.

Daphne Mezereum Lin. $\hbar$

\section{Tropaeolaceae.}

Tropaeolum majus L. $\odot$

\section{Umbelliferae.}

Aethusa cynapium L. $\odot$

Anethum graveolens L. $\odot$

Anthriscus cerefolium Hoffm. $\odot$

Archangelica officinalis Hoffm. - ;

Astrantia Biebersteinii Fisch. et Mey. 2

- helleborifolia Salisb. 24

- major L. 94

Athamantha Mathioli Wulf. Jacq. 24

Bupleurum falcatum L. ?

- graminifolium Wahl. 2

- longifolium L. 9

- rotundifolium Link. $\odot$

Carum Bulbocastanum Koch. 2

- Carvi L. $\odot$

Chaerophyllum aromaticum L. 4

- aureum L. 24

- bulbosum L. $\odot$

Cicuta virosa L. भ|

Coriandrum sativum L. $\odot$

Cryptotaenia canadensis Dec. ㅇ․

Daucus Carota L. 24

Eryngium agavifolium Griseb. 24

- amethystinum L. 2

- angustifolium L. 2

- bromeliifolium Laroche. 4

- coeruleum Bieb. 24

- dichotomum Desf. 24

- giganteum M. B. 24

- oliverianum Delar. 24

- planum L. 2

- rigidum Lam. 2

Ferula Ferulago L. 24

Foeniculum vulgare Gaert. (-)

Heracleum Lehmannianum Rgl. 4

- Sphondylium L. 24

Hydrocotyle moschata Forst. 2

- rotundifolia Roxb. 2

- vulgaris L. 2

Laserpitium intermedium L. 와

Levisticum officinalis Koch. 2/

- paludapifolium Lam. 2

Lophosciadum millefolium Dec. 4

Meum athamanticum Jacq. 4

Myrrhis odorata Scop. -)

Oenanthe Lachenalii Gmel. 2

- fistulosa L. 9

- peucedanifolia Pollich 24

- pimpinelloides L. 24

Pachypleurum alpinum Ledeb. 24

Pastinaca sativa L. 2f

Petroselinum sativum Hoffm. (-)

Peucedanum sylvestre Dec. 2

Pimpinella magna L. 24

- Saxifraga L. 24

Prangos thapsoides Dec. 4

Sanicula europaea L.

Siler aquilegifolium Gaertn. 24 
Sium angustifolium Koch. 24 - latifolium L. $भ$

Smyrnium Olusatrum L. 24

Trinia Hoffmani Bbrst. $\odot$

Zizia Kitaibelii Bbrst.

- integerrima Dec. 4

\section{Urticaceae.}

Dorstenia arifolia Lam. भ

Parietaria lusitanica L. $\odot$

Pilea muscosa Lindl. of

Urtica balearica L. $\odot$

- cannabina L. $\odot$

- macrophylla Thunbg. 2

- piluliferea L. $\odot$

\section{Valerianaceae.}

Centranthus Calcitrapa Dufresn. $\odot$

- macrosiphon Boiss. $\odot$

- ruber D. C. 2

Valeriana officinalis L. 와 - sambucifolia Mik. 2

Valerianella pumila Dec. $\odot$

\section{Verbenaceae.}

Verbena elegans H. B. 24

- officinalis L. 9

- paniculata L. 24

- urticifolia L. 2f

- venosa Gill. et Hook. 2

\section{Violaceae.}

Viola cornuta L. 2

- elatior Fries. 2

- mirabilis L. 2

- pinnata L. 2

- primulifolia L. 24

- tricolor L. $\odot$

\section{Zygophyllaceae.}

Peganum Harmala L. श Zygophyllum Fabago L. 24 


\section{Anhang.}

Pflanzen können abgegeben werden von folgenden Sedum- und Sempervivum-Arten :

Sedum acre L. 2

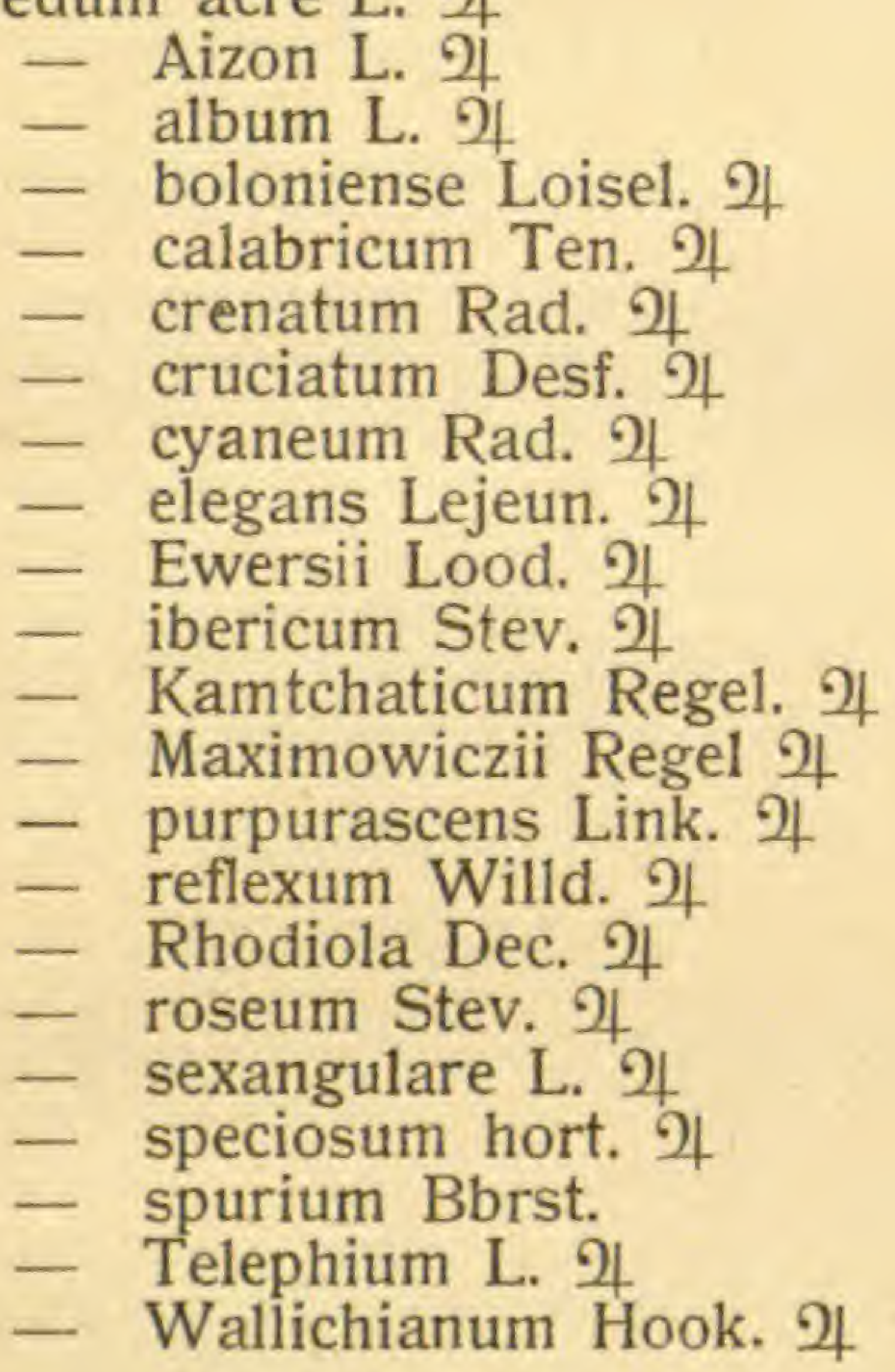

Sempervivum albidum Lehm. et Schnittsp. 2

- acuminatum Schott. 24

- arachnoideum L. 2

- arenarium Koch. 2

- arvernense Schott. 2

- assimile Schott. 2.

- blandum Schott. 2

- Boutignyanum Lk. et. Sch. 2

- Braunii Funk. 24

- californicum hort. 2

- canescens R. S. 24

- Comollii Rota. 2y

- cornutum Schott. 2

- debile Schott. 2

- Delastrii Lehm. et Schnittsp. 2

- dolomiticum Hausm. 2

- fimbriatum Lehm. et Schnittsp. 2
Sempervivum flagelliforme Fisch. 4

- Funkii A. Br. 2

- - brachypetalum A. Br. 2

- glaucum Ten. 2P

- globiferum L. 2

- grandiflorum Haw. 2

- Hausmannii Schott. 2

- heterotrichum Schott. 2

- Heuffelii Schott. 2

- hirtum L. 2

- hispidulum Schott. 2

- Laggeri Schott. 2

- Mettenianum Lehm. 2

- modestum Jord. 2

- montanum L. 2

- patens Griseb. 2

- pennicillatum Schott. 2

- piliferum hort. 2

- populifolium L. 2

- purpureum L. 9

- Reginae hort. 24

- Requienii R. S. 2

- robustum Himp. 24

- rubicundum Schur. 24

- ruthenicum hort. 9

- Schlehani Schott. 2

- Schottii Br. 24

- Schnittspahnii Dill. 24

- soboliferum Sims. 2

- spinulifolium hort. 24

- stenopetalum Lehm. et. Schnittsp. 24

- tectorum L. 2

- tomentosum Lehm. 24

- triste hort. 24

- Verloti Jord. 24

-

- - glaucum Jord. 2

- violaceum Blasius. 24

- Widderi L. 2

- Wulfenii Hopp. 2

Bestellungen werden bis zum 15. Februar erbeten.

Prof. Dr. M. Möbius

Direktor.
Rudolf Günther

Obergärtner. 


\section{varatitin}

(by $4=$

$15-7 \times 10$

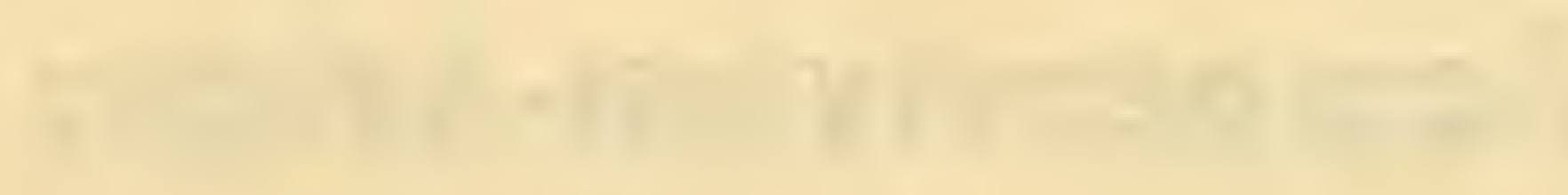
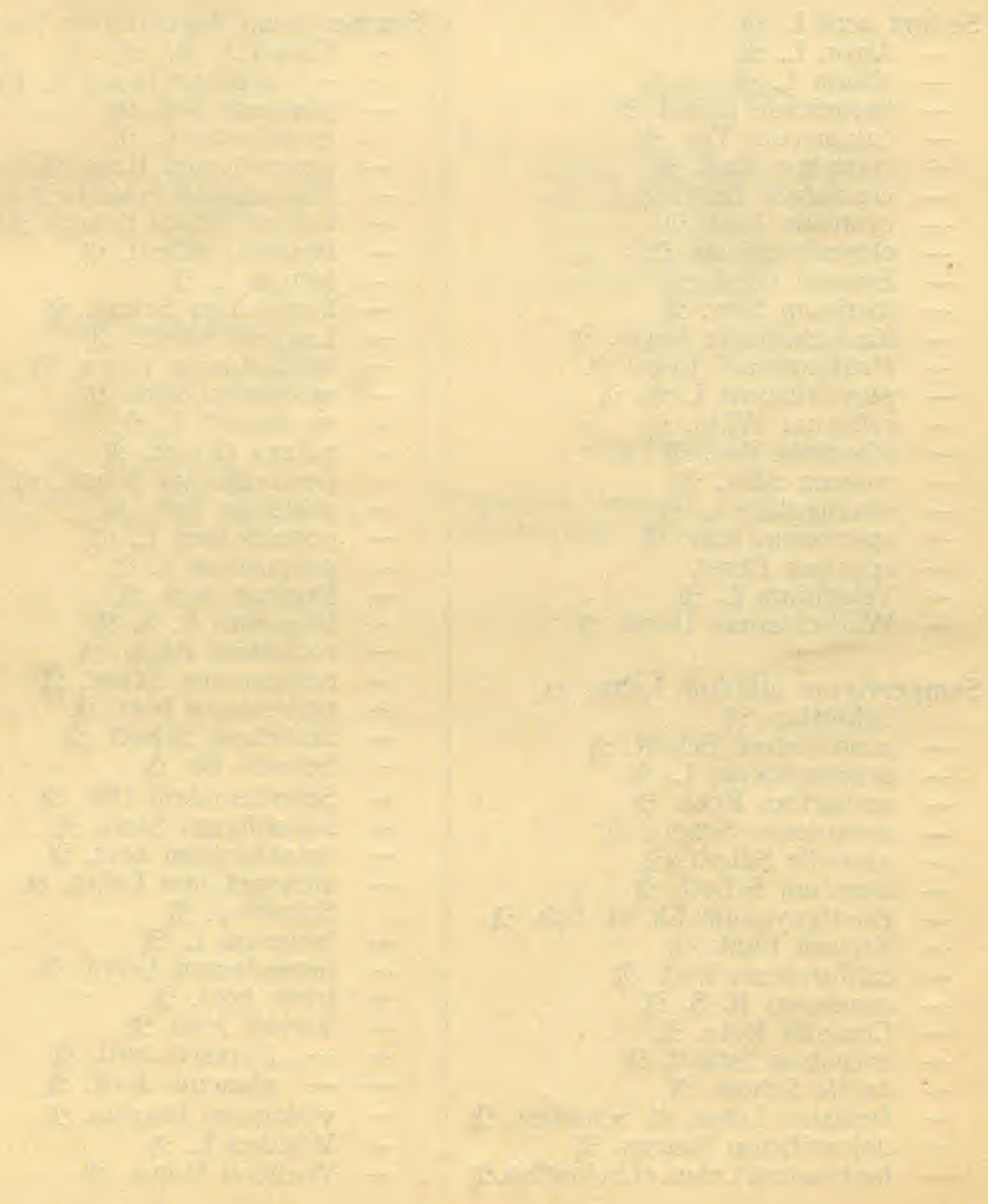

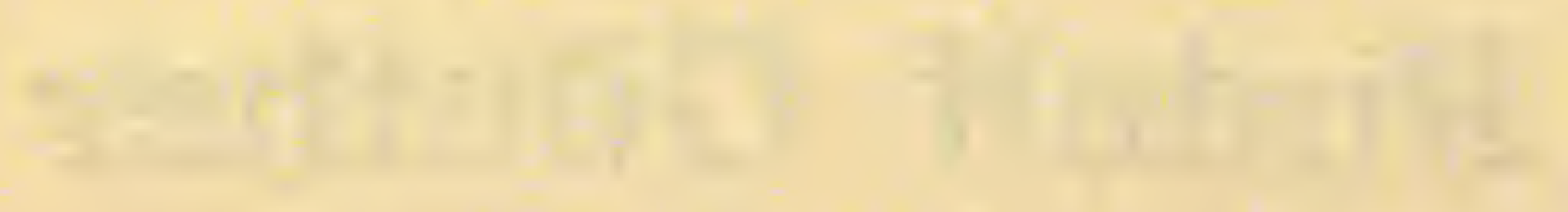

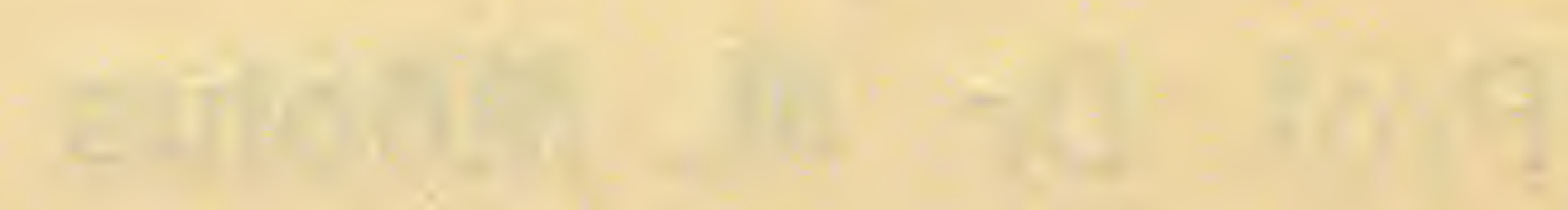
$3 x^{1}+a^{2}+y^{2}$ 




\section{SÄMEREIEN}

zum Tausch angeboten

vom

\section{Botanischen Garten in Frankfurt am Main. 1910.}

\section{Pteridophyta.}

\section{Filices.}

Acrostichum quercifolium Schrenk. Adiantum caudatum $\mathrm{L}$.

- Bausei L.

- crenatum Fisch.

- - var. gracillimum Moore.

- Edgeworthii Hook.

- formosum R. Br.

- gracillimum v. Houtte.

- grande Hort.

- hispidulum Sw.

- peruvianum Klotsch.

- polyphyllum Willd.

- rubellum Moore.

- scutum Hort.

- tenerum Sw.

- trapeziforme L.

- Weigandii Moore.

Alsophila australis $\mathrm{Br}$.

Aneimia laciniata Lk.

- Phyllitidis Sw.

- rotundifolia Sw.

Aspidium aculeatum Schwartz.

- angulare Kit.

- coriaceum Sm.

- Filix mas var. monstrosum Schwartz.

- Fortunei Sw.

- Goldianum Hook.

- remotum Al.

- trifoliatum Sw.
Aspidium violaceum Lk.

Asplenium adiantum nigrum $\mathrm{L}$.

- Belangeri Kze.

- diversifolium Blum.

- Goeringianum Moore.

- Nidus avis L.

- septentrionale Hoffm.

- Trichomanes L.

Athyrium alpestre Nyl.

- australe Prsl.

Blechnum brasiliense Desf.

- fraxineum Willd.

- gibbum Hook.

- giganteum Hort.

- longifolium H. B. K.

- occidentale L.

Ceratopteris thalictroides Brogn.

Davallia bullata Wall.

- canariensis Sm.

- dissecta L.

- Lehmanni Hort.

- platyphylla Don.

Dicksonia antarctica Labill.

Doodya media R. Br.

Gymnogramme chrysophylla Kze.

- Laucheana Hort.

- palmata Link.

- sulphurea Desf.

Hemionitis cordifolia Roxb.

- palmata L.

Hypolepis repens Presl.

Lygodium japonicum Sw.

Nephrolepis acuminata Kuhn.

- acuta Schk.

- Bausei Hort. 
Nephrolepis exaltata Schott.

Onoclea Struthiopteris Hoffm.

Ophioglossum vulgatum L.

Pellaea alternifolia Bak.

Platycerium alcicorne Desv.

- biforme Desv.

- grande Sm.

- Hillii Moore.

- Willinckii Moore.

Polybotrya aurita Blum.

Polypodium aureum v. glauca L.

- hexagonopterum Sw.

Pteris aquilina L.

- biaurita L.

- cretica var. albo-lineata L.

- decipiens Hook.

- ensiformis Burm.

- flabellata Thbg.

- gracilis Dec.

- heterophylla L.

- longifolia L.

- marginata B.

- palmata Willd.

- pedata L.

- quadriaurita Retz.

- serrulata var. cristata Veitch.

- tremula R. Br.

Scolopendrium daedaleum $\mathrm{L}$.

Todea barbara Moore.

- hymenophylloides Less.

Woodwardia angustifolia Sm.

- caudata Cav.

- radicans $\mathrm{Sm}$.

\section{Marsiliaceae.}

Marsilia Drummondii A. Braun. 24 - elata A. Braun. 24

\section{Psilotaceae.}

Psilotum triquetrum Sw.

\section{Selaginellaceae.}

Selaginella Braunii Bak.

- caulescens Sprg.

- cuspidata Link.

- Emmeliana Spring.

- flagellifera Bull.

- grandis Moore.

- helvetica Link.

- Martensii Spring

- pulcherrima Sieb.

- triangularis W.

- viticulosa Klotsch.

- Warscewiczii L.

- Willdenowii Back.

\section{Coniferae.}

Callitris verrucosa $\mathrm{R}$. Br. $\hbar$ Juniperus Oxycedrus L. $\hbar$ Juniperus Sabina L. $\hbar$ Picea laxa Sargent. $\hbar$ Sequoia sempervirens Endl. th Taxus baccata $\mathrm{L}$. $\hbar$

\section{Monocotyledoneae.}

\section{Alismaceae.}

Alisma Plantago L. 이 Sagittaria chinensis Sims, 아

- montevidensis Schlecht. 9

- sagittifolia Lin ข

\section{Amaryllidaceae.}

Agave Bouchei Jakobi. 2)

- Franzosinii Niss. 24

- mexicana Lam. 2|

Amaryllis nivea Schult. 2)

Cooperia Drummondi Hook. ㅇ

Haemanthus Nelsoni Jacq. 2f

- tigrinus Jacq. 24

Hippeastrum aulicum Herb. 2)

Zephyranthes atamasco Lin. ?

\section{Araceae.}

Anthurium Backeri Hook. ?

- magnificum Lind. 9|

- Scherzerianum Schott. 9f

Arum italicum Mill. ?

- maculatum L. 21

Sauromatum guttatum Schoth. 2f

Zantedeschia aethiopica Spreng. 91

\section{Bromeliaceae.}

Billbergia longifolia Lindl. 2)

- violacea Bert. ?

- Zebrina Lindl. 24

Caraguata lingulata Lindl. \%)

- Peacockii Moor. 2)

Dyckia remotiflora Otto Dietr. 2)

- sulphurea Lem. 24

- v. Houtte Lem. 21

Encholirion corallinum Mart. ?

Vriesea Megiana hort. ㄱ․

- Sandersii Morr. 2)

- viminalis Morr. 9f 


\section{Butomaceae.}

Butomus umbellatus L. 94

\section{Cannaceae.}

Canna indica L. ?)

\section{Commelynaceae.}

Commelyna coelestis Willd. 24 - tuberosa L. 24

Tinnantia erecta Sacg. 24

- fugax Schneider. 24

Tradescantia hypophaea L. 2)

- pilosa Lehm. 24

- virginica L. 24

- viridis L. 2)

\section{Cyperaceae.}

Carex glauca Scop. 24

- Grayana Dew. 24

- japonica fol. var. L. 24

- leporina L. 2

- maxima Scop. 21

- pendula Huds. 9

- sylvatica Huds. ข

- xanthophysa Wahlbg. 24

Cladium Mariscus R. Br. 24

Cyperus laxus Thbg. थै

- longus L. 24

- natalensis hort. थ

- Papyrus L. 24

- textilis Thbg. 24

Isolepis palustris Schrad. $(-)$

- prolifera R. Br. 24

Scirpus Holoschoenus Link. थ

- lacustris L. 2)

- - var. Zebrina L. 9

- maritimus L. 计

- radicans Schkuhr. 24

- sylvaticus L. 아

\section{Dioscoreaceae.}

Dioscorea alata Lin. 29

- japonica Thbg. 와

- verticillata Lam. 24

- villosa L. 2f

Tamus communis L. 9

\section{Gramineae.}

Alopecurus arundinaceus Poir. 2 Arundo Donax fol. varieg. Hort. 24
Avena barbata Roth. थ4

Bambusa spinosa Blum. 이

Brachypodium distachyum Beauv. $\odot$

Briza maxima L. 2

- media L. 24

Brizopyrum distachyum R. S. $\odot$

- siculum Link. $\odot$

Bromus arvensis Lin. $\odot$

- asper L. 2)

- erectus Huds. 21

- macrostachys Desf. $\odot$

- maximus Desf. $\odot$

Calamagrostis Epigaios L. 24

Chloris barbata Sw. $\odot$

Coix Lacryma L. $\odot$

Corynephorus canescens Beauv. 24

Dactylis glomerata L. 2)

Desmazierea sicula Dum.

Elymus arenarius L. 24

- giganteus Vahl. 24

- glaucifolius Muhlbg. 24

Festuca gigantea Vill. 2f

- glauca Lam. 24

- ovina L. 24

- pectinella Delil.

- rubra L. 2

- sylvatica Vill. 24

Hierochloa borealis R. S. 24

Hordeum distichum L. $\odot$

- maritimum With. $\odot$

Koeleria cristata Pers. $\hbar$

Lasiagrostis Calamagrostis Link. ?

Melica altissima Lin. थै

- ciliata Lin. 24

- exasperata R. S. 21

Miscanthus sacchariflorus Hack 24

Molinia coerulea Moench. 와

Nardurus unilateralis Boiss. $\odot$

Oryza sativa L. $\odot$

- - var. italica L. $\odot$

Panicum capillare L. $\odot$

- miliaceum L. $\odot$

- - var. album L. $\odot$

- $\quad-\quad$ nigrum Willd. $\odot$

- virgatum L. 24

Paspalum lentiferum Lam. 과

Pennisetum compressum R. Br. 와

- longistylum Hort. $\odot$

- villosum Br. $\odot$

Phalaris canariensis $\mathrm{L}$. $\odot$

- picta L. 2f

Phleum asperum Vill. $\odot$

Poa alpina L. 24

- bulbosa var. vivipara Lin. 24

Sorghum saccharatum Pers. $\odot$

Stipa capillata Lin. 24

- splendens Trin. 29

Triticum ciliatum Dec. $\odot$

- monococcum L. $\odot$ 
Triticum Spelta var. album L. $\odot$

Zea Mais L. $\odot$

- - var. caesia. $\odot$

- - Caragua Gmel. $\odot$

- - - cryptosperma Bonaf. $\odot$

- - - Jellow L. $\odot$

- - - Naurottolo L. $\odot$

- - - orizoides Kke. $\odot$

- - - rubra L. $\odot$

- - - tuscana L. $\odot$

- - - xanthomis Kke. $\odot$

\section{Iridaceae.}

Anomatheca cruenta Lindl. 24 Freesia refracta Klatt. 27 Iris lutescens Lam. 2)

- mauritanica Spr. 24

- Pseud-Acorus L. 24

- sibirica L. 2

- spuria L. 24

Ixia maculata L. 24

Sisyrinchium anceps Lam. 와

- Bermudianum L. 24

\section{Juncaceae.}

Juncus glaucus Sibth. 24

- squarrosus L. 2

- ulliginosus Roth. Sibth. 2f

Luzula albida Desv. 2)

- multiflora Desv. 24

- nivea D. C. 아

- pallescens Wahlbg. 24

\section{Juncaginaceae.}

Triglochin maritimus L. 요

- palustris L. $\odot$

\section{Liliaceae.}

Agapanthus africanus.

- africanus var. albiflora Voss. 24

- praecox Willd. 24

- umbellatus L'Herit. 24

Albuca fastigiata Dryant. 2

Allium cernuum Roth. 2f

- fallax Schult. 24

- fistulosum L. 29

- fragrans Vent. 24

- globosum M. B. 21

- Moly L. 21

- moschatum L. 2

- nigrum L. 24.

- nutans L. 24
Allium odorum L. 2)

- Porrum L. 2

- rotundum L. 2)

- strictum Schrad. 24

- sphaerocephalum Lin. 24

- ursinum L. 21

Aloe abyssinica Lam. 우

- Borziana Terr. 24

- glabra Salm. 24

- picta Lam. 24

Anthericum Liliago L. ข|

- ramosum L. of

Asparagus officinalis L. 讴

- Sprengeri Rgl. 24

Asphodelus albus Willd. 2)

- cerasifer J. Gay. 24

- fistulosus L. .)

Asphodeline liburnica Rchb. 24 - lutea L. 24

Bowiea volubilis Haworth. 24

Bulbine annua Willd. 24

Camassia esculenta Lindl. 24

Chlorophytum elatum R. Br. 24

Dasylirion acrotrichum Zucc. 24 - glaucophyllum Hook. 24

Dracaena concinna Kth. $\hbar$

- Draco L. $\hbar$

Eremurus albus L. 2)

- caucasicus Stev. 29

- himalaicus Baker. 2

- Olgae Rgl 아

- robustus Rgl. 2)

- spectabilis Ledeb. 2

- turkestanicus Rgl. 2)

Eucomis regia Ait. थ

- stricta Ait. 24

Fritillaria imperialis L. 24

- Meleagris L. ข|

- pyrenaica L. 2

- tenella Bbrst. 24

Funkia ovata Spr. 24

- Sieboldiana Lodd. थ

Galtonia candicans Desne. 의

Haworthia granata Haw. 이

Hemerocallis flava L 24

Hypoxis decumbens Lin. 2

Kniphofia Tuckii Bak. 24

- uvaria Hook. ขf

Libertia formosa Grah. 24

Lilium Martagon L. 24

- tigrinum Gawl. 24

Muscari comosum Willd. 2f

- racemosum Mill. 2|

Nothoscordum fragrans Kth. 24

Ornithogalum narbonense L. 24

Phormium Cookianum Lejolis. 24

- tenax Forst. 24

Polygonatum giganteum Otto et Dietr.2

- latifolium Desf.

- - var. commutata Schult. 4 
Polygonatum multiflorum All. 24

- officinale All. 24

- verticillatum Schult. 2

Puschkinia scilloides Adans of

Ruscus Hypophyllum L. 24

- racemosus L. 2

Scilla hispanica Mill. ?

- undulata Desf. 24

Smilax aspera L. 24

Smilacina racemosa Desf. थ|

- stellata Desf. ?

Tulipa Chrysolore Rgl. 24

- Didieri Jord. 2I

- Kolpakowskyana Rgl. ㄱ

- Marjolletii Perr. et Song. 2)

Uropetalum serotinum Ker. 24

Urginea maritima Bak. ข4

Veltheimia viridifolia Jacq. 9

Veratrum nigrum L. 와

Yucca aloifolia L. $\hbar$

Zygadenus bracteatus Schult. 2

- elegans Pursh. 2)

- glaberrimus Mxch. 24

- glaucus Nutt. 이

\section{Typhaceae.}

Typha angustifolia L. 24

- latifolia L. ?

- minima Funk. 24

\section{Dicotyledoneae.}

\section{Acanthaceae.}

Acanthus longifolius Poir. 24

- mollis L. 24

- niger Mill. Link. 9

Ruellia Devosiana hort. 4

- strepens L. 24

\section{Aizoaceae.}

Mesembrianthemum argenteum hort. 24

- barbatum L. 2

- crystallinum L. $\odot$

- glaucum L. $\hbar$

- heteropetalum Haw. 2

- Lehmanni Eckl. $\odot$

- multiflorum Haw. $\hbar$

- pinnatifidum Lin. $\odot$

- spectabile Haw $\hbar$

- tigrinum Haw. 9

- tricolor Haw. $\odot$

Mollugo verticillata $\mathrm{L}$. $\odot$

Tetragonia crystallina L'Hérit.

\section{Amarantaceae.}

Amarantus albus L. $\odot$

- caudatus L. $\odot$

- - fl. rubro L. $\odot$

- fl. flavo L. $\odot$

- hypochondriacus L. $\odot$

- paniculatus L. $\odot$

- speciosus L. $\odot$

- - v. monstrosa L. $\odot$

Celosia cristata L, $\odot$

- - var. aurantiaca L.

- - - plumosa L. $\odot$

- cristata var. splendens

- pyramidalis plumosa hort. $\odot$

Gomphrena globosa L. $\odot$

- alba L. $\odot$

- Haageana KI. $\odot$

\section{Anacardiaceae.}

Rhus vernicifera DC. $\hbar$

\section{Apocynaceae.}

Amsonia angustifolia Michx. ')

- latifolia Michx. 2)

- Tabernaemontana Walt. 24

- salicifolia Pursh. 9f

Apocynum cannabinum L. 9

Nerium Oleander L. 24

\section{Araliaceae.}

Acanthopanax sessiliflorum Seem. $\hbar$ Aralia Sieboldii hort. $\hbar$

- spinosa L. $\hbar$

\section{Aristolochiaceae.}

Aristolochia altissima Desf.

- fimbriata Chm. 24

- gracilis Elliot. भy

Asarum canadense L. 2)

- europaeum L. 9

\section{Asclepiadaceae.}

Asclepias fruticosa L. ?)

- incarnata L. 24

- mexicana Cav. 21

- speciosa Torr. 24

- syriaca L. 24 
Asclepias verticillata L. 24

- virgata Balb. 24

Cynanchum furcatum Rchbch. 24

- nigrum Moench. ?)

- Vincetoxicum L. 2)

\section{Balsaminaceae.}

Impatiens Balsamine L. $\odot$

- cristata W. $\odot$

- fulva Nutt. $\odot$

- glanduligera Royl. $\odot$

- longicornis Wall. $\odot$

- Roylei Walp. $\odot$

- scabrida Dec. $\odot$

\section{Begoniaceae.}

Begonia gracilis Jacq. थ4

- semperflorens Lk. et Otto. 24

\section{Berberidaceae.}

Podophyllum Emodi Wahl

\section{Bignoniaceae.}

Incarvillea Delavayi Franch. 와

- Olgae Rgl. 9|

- sinensis Lam.

Sesamum indicum L. $\odot$

Tecoma australis $\mathrm{R}$. Br. $\hbar$

- Jasminoides Lindl. $\hbar$

\section{Borraginaceae.}

Anchusa italica Retz. ?)

Borrago officinalis L. $\odot$

Caccinia strigosa Boiss. 2)

Cerinthe retorta Sib. $\odot$

Echinospermum macranthum Ledeb. $\odot$

Echium lusitanicum Lin $\odot$

Heliotropium europaeum L. $\odot$

Lithospermum apulum Lin. $\odot$

-- officinale L. 2)

Myosotis palustris L. ?/

Omphalodes linifolia Moench. $\odot$

Symphytum asperrimum Sims. 2)

- bohemicum Schmidt. 2)

- Carmosina Hort. 2)

- caucasicum M. B. 2)

- officinale L. 24

- - fl. violaceo L. ข

\section{Cactaceae.}

Mamillaria rhodantha Lk. et Otto. 9)

Rhipsalis sarmentacea Otto et Dietr. ㅇ

\section{Caesalpiniaceae.}

Caesalpinia coriaria Willd. $\hbar$. - mexicana A. Gray. $\hbar$ Cercis Siliquastrum L. $\hbar$

\section{Calycanthaceae.}

Calycanthus occidentalis Hook. $\hbar$ Chimonanthus fragrans Lindl. $\hbar$

\section{Campanulaceae.}

Campanula alliariifolia Willd. 24

- latifolia Fisch. 9y

- Medium var. alba L. $\odot$

- - - - plena L.. .

- - - calycantha L. $\odot$

- $\quad$ - coerulea L. $\odot$

- - - - plena L. $\odot$

- - - rosea L. $\odot$

- - - plena L $\odot$

- nobilis Lindl. 9

- persicifolia var. coerulea L. 2

- - L. 9

- peregrina L. 24

- rapunculoides L. 2)

- rotundifolia L. 9

- Scheuchzerii Vill. 2f

- strigosa Vahl. $\odot$

- thyrsoidea Lin. 24

- Trachelium L. 2f

- turbinata Schott. 24

- urticifolia Lam. 2)

- Vidalii Wats. 24

Jasione perennis Lam. ?f

Musschia Wollastonii Lowe. 2f

Phyteuma Scheuchzeri All. 24

Piddingtonia Palliardii Lem. 2)

Platycodon autumnale Dcsne. 24 - grandiflorum DC. थ

Siphocampylus foliosus L. $\odot$

Specularia falcata Dec. $\odot$

- salina Pres1. $\odot$

- Speculum DC. $\odot$

Trachelium coeruleum Lin. 29

Wahlenbergia nutabunda Dec. $\odot$

\section{Cannabaceae.}

Cannabis sativa L. $\odot$

Humulus Lupulus L. .

\section{Capparidaceae.}

Cleome pungens Willd. $\odot$ - spinosa Jacq. $\odot$

Polanisia graveolens Rafin. $\odot$ 


\section{Caricaceae.}

Vasconcellea quercifolia St. Hil. $\hbar$

\section{Caryophyllaceae.}

Agrostemma Githago L. $\odot$

- nicaeensis Willd. $\odot$

Arenaria peploides Ehrh. 2

Cucubalus baccifer Lin. 24

Dianthus barbatus L. 9

- Carthusianorum Lin. 24

- caesius Sm. 2

- cruentus Griseb. 29

- deltoides L. 2

- plumarius L. 94

- superbus L. 24

- sylvestris Wulf. 9

Drypis spinosa L. $\odot$

Frankenia pulverulenta Lin. $\odot$

Gypsophila acutifolia Fisch. 9|

- altissima L. 24

- cerastioides Don. 2

- elegans Bbrst. $\odot$

- paniculata L. $2 \mid$

- perfoliata L. 9

- porrigens Boiss. 24

- prolifera L. 24

- repens L. 2

Herniaria glabra L. 24

- hirsuta L. 2|

Lepigonum marginatum Koch. ')

Lychnis calcedonica L. 나

- diurna Sibth.. .

- Flos Jovis Lam. 24

- Flos Cuculi L. 24

- Haageana Lem 24

Sagina apetala L. $\odot$

- pilifera L. $\odot$

- subulata Sw. $\odot$

Saponaria officinalis L. 가

Silene alpestris Jacq. ?|

- Armeria L $\odot$

- Coeli-rosa Rhrb. $\odot$

- Drummondi Hook. 9

- fimbriata Sims. 24

- glauca Zea. $\odot$

- Hornemanni Steud 2

- muscipula L. $\odot$

- nemoralis W. et K. 29

- obtusifolia W. 24

- paradoxa Lin 4

- pendula L. $\odot$

- quinquevulnera L. $\odot$

- saxatilis Bbrst. 2|

- syriaca Boiss. 24

- viridiflora L. 2

- Zawadskyi Presl 24

Tunica prolifera Scop. भ|

- saxifraga Scop. $2 /$
Viscaria alpina Fries, 2

- Sartorii Boiss. 24

- viscosa Aschers. 24

- viscosa var splendens fl, pl. Aschers. 2|

\section{Casuarinaceae.}

Casuarina equisetifolia Forst. it

- torulosa Ait. $\hbar$

\section{Chenopodiaceae.}

Atriplex hortensis L. $\odot$

- laciniata L. $\odot$

- rosea L. $\odot$

Basella ramosa Jacq. $\odot$

Blitum virgatum L. $\odot$

Chenopodium album L. $\odot$

- Bonus Henricus L. 24

Hablitzia tamnoides Bbrst. ?f

Kochia hyssopifolia Roth. $\odot$

- scoparia Schrad. $\odot$

- trichophylla L. $\odot$

Salsola crassa Bib. $\odot$

- Kali L. $\odot$

Spinacia glabra Mill. $\odot$

\section{Cistaceae.}

Cistus populifolius L. $\hbar$

- salicifolius L. $\hbar$

- salvifolius L. $\hbar$

Helianthemum grandiflorum DC, 2

- ledifolium Pers. $\odot$

-. polifolium Pers. 9

- salicifolium Pers $\odot$

- umbellatum Mill. $\hbar$

- variabile Sw. 24

\section{Compositae}

Achillea ageratifolia v. Airon Bth. of

- cartilaginea Ledeb. 24

- lanata Spr. ?t

- Millefolium L. 24

- nobilis L 24

- Ptarmica L. 24

- $\quad$ fl. pl. L. 24

Acroclinium roseum Hook. $\odot$

Actinomeris tetraptera Dec. of

Agathaea spatulata L. 아

Ageratum mexicanum Swei $\odot$

Alfredia cernua Cass 24

Anthemis austriaca Jacq. ?| 
Anthemis tinctoria L. ?]

Arctium japonicum Hort. थ|

Arnica montana Lin. 2)

Artemisia Absinthium L. ?t

- annua L. $\odot$

- campestris L. 2

- Dracunculus L. 24

- Ludwigiana Nutt. 2)

Aster alpinus L. 24

- amellus L. 2)

- himalayensis Hook. 2)

- Linosyris Bernh. 2)

Barkhausia rubra Moench ?

Bidens leucantha Willd. $\odot$

Biotia commixta Dec. 2)

- macrophylla Dec. 24

- latifolia Dec. 2)

Cacalia suaveolens L. 2)

Calais Bigelowii A. Gray. $\odot$

Calendula officinalis L. (-

Calliopsis bicolor Rchbch. ()

- cardaminifolia Dec. $\odot$

Cardopatium corymbosum Pers. 2)

Carlina acaulis L. 2y

Carthamus tinctorius L. $\odot$

Cenia turbinata Pers. $\odot$

Centaurea alpina L. 2)

- calocephala Willd. 2

- candidissima Lam. 9)

- coronopifolia Lam. 2)

- Cyanus L. $\odot$

- imperialis alba L. $\odot$

- Kotschyana Heuff. of

- macrocephala Pusk. .)

- margaritacea Tenor. 2)

- melitensis L. $\odot$

- pulcherrima Willd. 2)

- Rhaponticum L. 24

- Scabiosa L. 2

- strobilacea Scop. $\odot$

- suaveolens Willd. $\odot$

Chamaepeuce diacantha DC. $\odot$

Chrysanthemum Balsamita L. .)

- carinatum Schrad. $\odot$

- corymbosum L. 2)

- Leucanthemum L. .)

- roseum Web. et Moor. -)

- vulgare Bernh. 2)

Chondrilla juncea L. 2)

Cirsium canum All. 아

- heterophyllum All. 2)

Cnicus benedictus L. $\odot$

Coreopsis cardaminifolia Dec. $\odot$

- longipes Hook. 2y

- palmata Nutt. 2)

- tinctoria Nutt

Cosmos sulphureus Cav. $\odot$

Crepis latifolia Ball. 2)

- longifolia Ball. 2)

- pulchra L. $\odot$
Crepis rubra L. $\odot$

- sibirica L. ข

Cryptostemma hypochondriac: R.Br. $\odot$

Cynara Cardunculus L. $\odot$

Dahlia coccinea Cav. 2)

- variabilis Desf. 24

Dimorphotheca pluvialis Moench. $\odot$

Doronicum caucasicum Bbrst. 의

- marcrophyllum Fisch. 2f

- Pardalianches L. थ

Echenais carolinoides Cass. $(-$

Echinops dahuricus Fisch. $\odot \mid$

- globifer Frank. 24

- humilis Bieb. 24

- sphaerocephalus L. $)$

Endoptera Dioscoridis Dec. $\odot$

Erigeron speciosus Dec. ?)

Ethulia conyzoides Lin. $\odot$

Eupatorium aromaticum L. 2)

- ageratoides L. 24

- cannabinum L. 2)

- Haageanum Regel. et Kern. 2

- heterophyllum Dec. 2)

- grandiflorum Dec. 24

- purpureum L. 2

- Purpusii Brand. 24

Gaillardia aristata Pursh. श)

- cyaniflora Hort. 2f

- lanceolata Michx. 2

- maxima L 2)

- pulchella Foug. $\odot$

Geropogon glaber L $\odot$

Gnaphalium purpureum L of

Grindelia arguta Schrad. 2f

- integrifolia Dec ?

Haplopappus croceus A. G. 2)

Hedypnois persica Bbrst. $\odot$

Helianthus annuus L. $\odot$

Helichrysum bracteatum Willd. $\odot$

- elegans Don. 24

Helminthia echioides Gaert.

Hieracium alpinum L. 2)

- aurantiacum L. .)

- Auricula L. 94

- amplexicaule L. ข|

- dentatum Hoppe. -)|

- eriophorum Link. 9)

- Lactaris Bert. 2)

- lycopsifolium Froel. -9

- pulmonarioides Vill. 24

- ramosum Suter. 24

- sabandum L. 2)

- sylvestre Tausch. 9|

- villosum Link. ?

Humea elegans Sm. $\odot$

Hypochoeris glabra L. $\odot$

Inula germanica $\mathrm{L}$. ?)

- glandulosa Willd. 2f

- Helenium L. 24

- hirta L. 2f 
Inula media Bbrst. 24

- saligna L. 2

- thapsoides L. 2)

Iva xanthifolia Nutt. $\odot$

Kentrophyllum lanatum Dec. $\odot$

Krigia caroliniana Nutt. $\odot$

Lactuca perennis L. 2)

- saligna L. $\odot$

- Scariola L 24

- virosa L. 9)

Lagascea mollis Cav. $\odot$

Lampsana communis L. $\odot$

- grandiflora Bbrst. $\odot$

Lappa officinalis All. $\odot$

Leontopodium alpinum Karst. 24

- himalayanum Dec. 24

Lepachys pinnatifida liafin. 와

Ligularia marcrophylla Dec. 24

- sibirica Cass. 24

- speciosa F. M. 24

- thyrsoidea Dec. 와

Linosyris vulgaris Dec. थै

Lonas inodora Gaert. $\odot$

Madaria elegans Dec. $\odot$

Madia sativa Molin.

Maruta Cotula Bbrst.

- lithuanica Bess.

Matricaria Chamomilla L. $\odot$

Mulgedium macrophyllum Dec. ?)

- palustre L. 24

- sibiricum Less. 24

Onopordon Acanthium L. $\odot$

- elongatum L. $\hbar$

- macranthum Clarke. $\odot$

Palafoxia texana Hook. $\odot$

Parthenium integrifolium L. 2)

Picris dahurica Pursh. $\odot$

- hieracioides Lin. 24

Prenanthes purpurea L. 24

Ptarmica alpina L. 29

- speciosa Dec. 29

Pulicaria dysentrica Gaert. 24

- odora Rchbch. 24

Pyrethrum corymbosum Willd. 24

- eximum Dec. 아

- macrophyllum W. 24

- Parthenium Smith. 24

- roseum Bbrst. 2y

- Tchihatchewii Voss. 24

Rhagadiolus caudatus L. $\odot$

- stellatus Gaert. $\odot$

Rhaponticum scariosum Lam. 이

Rudbeckia digitata Mill. 24

- columnaris Pursh 24

- laciniata L. 24

- speciosa Wender. ข|

- moschata Lood. थ

Santolina Chamaecyparis $\mathrm{L} \hbar$

- rosmarinifolia L. $\hbar$

Sanvitalia procumbens Lam. $\odot$
Saussurea discolor DC. ?|

Scolymus maculatus L. $\odot$

Scorzonera villosa Scop. 2

Senecio artemisiifolius Pers. 24

- crispus var. rivularis Dec. 24

- Doria L. 94

- Jakobaea L. $\odot$

- marcrophyllus M. B. ㄱ

- nemorensis Jacq. 24

- Fuchsii Gmel. 24

- palustris L. $\odot$

Serratula coronata L. $\%$

- gigantea L. ?4

- heterophylla Desf. 24

- radiata Bbrst. 이

- tinctoria L. 2

Seriola aethnensis L. $\odot$

Silphium integrifolium Mchx. 24

- laciniatum L. 24

- perfoliatum L. 2

- trifoliatum L. 24

Silybum Marianum Gaertn. $\odot$

Solidago canadensis L. ?/

- lanceolata Ait. 구

- serotina Ait. 24

- verrucosa Schrad. 24

Spilanthes oleracea L. $\odot$

Stevia laxiflora Dec. ?|

Ursine anthemodes B. Hgp. $\odot$

Verbesina enceliodes B. Hgp. $\odot$

Viguiera prostrata Dec. 24

Xanthium macrocarpum Dec. ㅇ․

- spinosum L. $\odot$

- Strumarium L. $\odot$

Xeranthemum cylindricum Smith. $\odot$

Xanthocephalum gymnospermoides Benth. 2)

Zacyntha verrucosa Gaert. $\odot$

Zaluzania triloba Pers. 와

Zinnia Haageana Klotsch.

- multiflora L. $\odot$

- tubulosa hort. $\odot$

- verticillata Andr. $\odot$

\section{Convolvulaceae.}

Convolvulus arvensis L. 2f

- pentapetaloides L. $\odot$

- pseudosiculus Cav. $\odot$

- Scammonia L. 24

- tricolor L. $\odot$

Cuscuta europaea L. $\odot$

- glomerata Choisy. $\odot$

- planiflora Ten. $\odot$

Ipomaea coccinea L. $\odot$

- Nil Rth. $\odot$

- Purga Wender. 24

- purpurea Lam. $\odot$

- tricolor Cav. $\odot$ 


\section{Coriariaceae.}

Coriaria myrtifolia $\mathrm{L} . \hbar$

\section{Cornaceae.}

Benthamia fragifera Lindl. $\hbar$

\section{Crassulaceae.}

Bryophyllum ealycinum Salisb. ㅇ

- crenatum Backer. 24

Crassula falcata Ait. 24

- lactea Ait. 2)

- perfoliata Ait. 9

Sempervivum tabulaeforme Haw. 24

Umbilicus oppositifolius DC. 구

- pendulinus DC. 24

- Pestalotzii DC. 2

\section{Cruciferae.}

Aethionema grandiflorum Boiss et Hohen. 2)

- pulchellum Boiss. et Hohen. 24

Alyssum alpestre L. 2)

- argenteum All. 9|

- calycinum L. $\odot$

- edentulum W. et K. 24

- minimum Willd. $\odot$

- saxatile L. 21

Anastatica hierochuntica L. $\odot$

Arabis albida Stev. 24

- hirsuta Scop. $\odot$

Aubrietia erubeseens Gaert. 21

- graeca Griesb. 24

- grandiflora hort. 24

Barbarea arcuata Rchbch. $\odot$

- aurea R. Br. 24

- orthoceras Ledeb. 2|

- vulgaris R. Br. 2)

Berteroa incana Dec. ơ

Biscutella apula L. $\odot$

- auriculata L. $\odot$

- eriocarpa Dec. $\odot$

Brassica nigra Koch. $\odot$

- oleracea L. $\odot$

- - var. acephala L. $\odot$

- - bullata L. $\odot$

- - plumosa L. $\odot$

Bunias Erucago L. $\odot$

- orientalis L. $\odot$

Cakile maritima Scop. $\odot$

Camelina microcarpa Andriz. $\odot$

- sativa Crantz.

Cardamine pratensis L. Of

Cheiranthus Cheiri L. $\odot$

Cochlearia danica L. $\odot$

Crambe cordifolia Stev. of

- grandiflora Dez. थ।
Crambe hispanica L. $\odot$

- maritima L. ข

- orientalis L. ข|

- pinnatifida R. Br. 24

Diplotaxis tenuifolia DC. 24

Draba aizoides Lin. 24

- contorta Ehrh. 9f

- Ruyschiana L. 2|

- stellata Jacq. 아

Eruca capadocica L. $\odot$

Erysimum aureum Bbrst. $\odot$

- orientale Fisch. et May. $\odot$

- perfoliatum Crantz. $\odot$

- Perofskianum Fisch. et May.

- pulchellum Willd. 2

Farsetia clypeata R. Br. $\odot$

Heliophila amplexicaulis Lin. $\odot$

Hesperis fragrans Fisch. 아

- matronalis L. 2|

Hymenophysa pubescens Mayer. 24

Iberis amara L. $\odot$

- Garrexiana All. †

- pinnata L. $\odot$

- saxatilis L. 2

- sempervirens L. 이

- umbellata L. $\odot$

Isatis alpina L. थ

- dasycarpa Ledeb. $\odot$

- tinctoria L. $\odot$

Jonopsidium acaule Rchb. $\odot$

Lepidium crassifolium W. K. 24

- latifolium L. ㅇ

- sativum L. $\odot$

Lunaria annua L. $\odot$

Malcolmia chia Dec. $\odot$

- maritima R. Br. $\odot$

Moricandia hesperidiflora Dec. $\odot$

Myagrum perfoliatum L. $\odot$

Nasturtium amphibium R. Br. 24

- officinale $\mathrm{R} \mathrm{Br}$. 2)

Raphanus caudatus L. $\odot$

Ricotia Lunaria D. C. $\odot$

Senebiera Coronopus Poir. $\odot$

- didyma Pers. $\odot$

Sinapis abyssinica L. $\odot$

- alba L. $\odot$

- arvensis L. $\odot$

Sisymbium Irio L. $\odot$

- strictissimum L. 94

- tanacetifolium L. 2)

Zilla myagroides Forsk. of

\section{Cucurbitaceae.}

Bryonia alba L. 와

- dioica Jacq. 2)

Citrullus Colocynthis Schrad. $\odot$

Cucurbita ficifolia Wall. $\odot$

- Pepo var. annulata hort. $\odot$ 
Cyclanthera explodens Naud. $\odot$ Ecballium Elaterium L. $\odot$ Kedrostis africana Cogn. 2 Lagenaria vulgaris Ser. $\odot$ Luffa acutangula Roxb. $\odot$ - cylindrica $\mathrm{L}$. $\odot$

Melothria punctata Cogn. 2) Micrampelis lobata O. Katz. ') Momordica Charantia L. $\odot$ - Huberi Tod. $\odot$

- involucrata E. May. $\odot$

Sicyos angularis L. $\odot$

Thladiantha calcarata Clarke. 24

\section{Datiscaceae.}

Datisca cannabina L. ?)

\section{Dipsacacae.}

Cephalaria ambrosioides R. S. थ)

- centaureoides R. S. ?)

- joppensis Coult. $\odot$

- laevigata Schrad. 2y

- melitensis L. $\odot$

- tatarica R. S. 24

Dipsacus fullonum Mill. -(

- laciniatus L. $\odot$

- sylvestris Mill. $\odot$

Morinia longifolia Wall. 2

Pterocephalus palaestinus Coult. $\odot$

Scabiosa atropurpurea Desf. $\odot$

- caucasica Bbrst. 2/

- graminifolia L. 2)

- ochroleuca L. 9)

- palaestina L. $\odot$

- prolifera L. $\odot$

\section{Empetraceae.}

Empetrum nigrum L. $\hbar$

\section{Ericaceae.}

Rhododendron arboreum Sm. $\hbar$.

- ferrugineum L. $\hbar$

- hirsutum L. †.

\section{Euphorbiaceae.}

Croton montevidensis Spr. Euphorbia cyathophora Jacq.

- Engelmanni Bois.

- Esula L 24

- Gerardiana Jacq. 24

- globosa Sims. 24

- Lathyris L. 2)

- Myrsinitis L. 24

- nicaeensis All. 21

- orientalis L. 2)
Euphorbia Peplus L. $\odot$

- trigonocarpa Lodd. .)

- verrucosa L. 2

Manihot carthaginensis Muell. $\hbar$

Mercurialis annua L. $\odot$

Phyllanthus juglandifolius Willd. $\hbar$

- maderaspatensis $\mathrm{L}$. $\hbar t$

Ricinus communis L. $\odot$

- tunicensis Desf. $\odot$

- Zanzibariensis L. $\odot$

\section{Fagaceae.}

Quercus Ilex L. †

- lusitanica Lam. $\hbar$

- Robur Willd. $\hbar$

- Suber L. †

\section{Gentianaceae.}

Gentiana acaulis L. ?)

- cruciata L. 2

- lutea L. 2y

- Olivıeri Griseb. 2)

- punctata L. 2)

- tibetica King. 2/

- verna L. 2|

Limnanthemum geminatum Griseb.

Menyanthes trifoliata L. .)

Swertia perennis L. 2

\section{Geraniaceae.}

Erodium gruinum Willd. $\odot$

Geranium aconitifolium L. 2/

- canescens Herit. ?t

- cristatum Stev. 94

- ibericum Cav. 9f

- palustre Lin. 2

- pratense fl. albo L. 24

- sanguineum L. 2)

- sylvaticum L. 2

- Vlassowianum Fisch. 24

Pelargonium coronopifolium Jacq. 24

- cuculatum Ait. 2)

- tomentosum Jacq. $\hbar$

\section{Gesneriaceae.}

Ramondia Myconi F. Schulz. 구 Streptocarpus caulescens Hook. 2

- kewensis Hook. 24

- Veitchii hort. 24

- Wendlandii L. 24

\section{Globulariaceae.}

Globularia bellidifolia Ten. 2f

- cordifolia C. .

- vulgaris L. 4 


\section{Halorrhagidaceae.}

Gunnera chilensis Lam. ๑)

- manicata Lind. 24

Halorrhagis alata Jacq. $\hbar$

Hippuris vulgaris L. थै

\section{Hydrophyllaceae.}

Nemophila insignis Doug1. $\odot$

Phacelia tanacetifolia Benth. $\odot$

- viscida Torr. $\odot$

Wigandia caracasana H. B. et Kth.

\section{Hypericaceae.}

Hypericum Androsaemum L. 2)

- calycinum L. $\hbar$

- floribundum Ait. 24

- hircinum L. 9

- grandiflorum Salisb. 24

- olympicum L. $\hbar$

- perforatum L. 9|

- pulchellum L. 2

\section{Juglandaceae.}

Pterocarya fraxinifolia Spach. $\hbar$

\section{Labiatae.}

Ballota nigra L. ?|

Betonica hirsuta L. ?

- officinalis L. 9

- stricta Ait. 2)

Calamintha Nepeta Link. '

Elscholtzia cristata Willd. $\odot$

Hyssopus officinalis L. 24

- - fl. albo L. 2

Hyptis pectinata Poit. 94

- spicigera Lam

Lamium album L. ?

- garganicum L. ข|

- Orvala L. 24

Lavandula officinalis Cheix. ?

- vera Dec. 24

Leonurus capitatus L. 9)

- Cardiaca Adans. 9

- sibiricus L. $\odot$

Lophanthus anisatus Benth. th

Lycopus exaltatus L. ?f

Marrubium vulgare L. ㅇ

Melissa Acinos Benth $\odot$

- officinalis L. 2

Mentha crispa Alef. ?

$$
\begin{aligned}
& \text { - piperita L. 24 } \\
& \text { = Pulegium L. } 94 \\
& \text { - sylvestris L. } 24
\end{aligned}
$$

Monarda didyma L. ข|

- fistulosa L. 24

- mollis L. 2

Molucella spinosa Lin. $\odot$

Nepeta cyanea Steven. 9

- grandiflora M. B. .

- latifolia DC. 24

- tuberosa L. 2

Origanum vulgare L. 2

Perilla nankinensis Voss. $\odot$

Phlomis tuberosa L. 24

Physostegia virginica Benth. 2)

Plectranthus fruticosus L'Herit. 24

Prunella grandiflora L. ขै

- vulgaris L. 2)

Salvia Aethiopis L. $\odot$

- argentea L. $\odot$

- dumetorum Andr. 2

- glutinosa L. P

- officinalis L 9

- Sclarea L. 9|

- sylvestris L. .)

- verticillata L. 2

- _ fl. albo L 24

Satureja variegata Host. थ|

Scutellaria alpina L. 24

- Lupulina L. 91

Sideritis montana L. 2

Stachys circinnata L'Herit. 2

- germanica L. 24

- lanata Jacq. 2)

- longifolia Benth. 2/

- palustris L. 24

- recta L. ?

- setifera C. A Meyer. 24

- sylvatica L. 94

Teucrium Botrys L. $\odot$

- Chamaedrys Lin.

- Scorodonia L. 9

Thymus vulgaris L. थ

\section{Lauraceae.}

Persea carolinensis Nees. $\hbar$

\section{Linaceae.}

Linum alpinum L. 94

- angustifolium Huds. 2|

- aquilinum Dec. $\odot$

- arborescens L. 24

- austriacum L. 2

- corymbiferum Desf. 9

- cribrosum L. $\odot$

- flavum L. 2]

- grandiflorum Desf. $\odot$

- perenne L. 24

- usitatissimum L. $\odot$ 
Loasaceae.

Blumenbachia Hieronymi Urb. $\odot$

- insignis Schrad. $\odot$

Cajophora lateritia Presl.

Loasa papaverifolia H. B. Kth. $\odot$

- tricolor Lindl.. .

- triphylla Juss. $\odot$

- vulcanica Andre.

Lobeliaceae.

Lobelia syphilitica L 2

- urens L. $\odot$

\section{Loranthaceae.}

Viscum album L. $\hbar$

\section{Lythraceae.}

Cuphea Llavea Llav, et Lex, .) - petiolata L. 2

Heimia salicifolia Link. $\hbar$ - syphilitica D C. 의

Lythrum Grafferii Ten. 24

- lineare L. 2f

- Salicaria L. 2.

- - var. roseo-superba L. 아

- tomentosum D C. 24

\section{Malvaceae.}

Abelmoschus esculentus Mey. $\odot$ Abutilon hirtum Don. $\odot$

- Sonneratianum Cav. $\odot$

- tiliaefolium Fisch.

Althaea cannabina L. 9

- officinalis L. 2)

- rosea Cav. 2.

- - fl. albo Cav.

- - v. monstrosa Cav. $\odot$

- - fl. purpureo Cav. $\odot$

- - fl. nigro Cav. $\odot$

- taurinensis Dec. 2)

Anoda hastata Cav. $\odot$

Gossypium arboreum L. †

- herbaceum L. $\odot$

Hibiscus esculentus L. $\odot$

- syriacus L. $\hbar$

- Trionum L. $\odot$

Kitaibelia vitifolia Willd. $\odot$

Lavatera trimestris L. $\odot$

Malachra alceaefolia Jacq. $\odot$

Malope malacodes L. $\odot$ - trifida Cav. $\odot$

Malva caroliniana crispa L. $\odot$

- crispa L. $\odot$

- involucrata Torr et Gr. 24

- mauritiana L. $\odot$
Malva nicaeensis All. $\odot$

Sida frutescens Cav. $\hbar$

\section{Mimosaceae.}

Acacia albida Del. $t$

- floribunda Willd. $\hbar$

- latifolia Benth. $\hbar$

- linifolia Willd $\hbar$

- longifolia Willd. to

- lophantha Willd. $\hbar$

Albizzia Julibrissin Bow. $\hbar$

Calliandra coccinea Dec. $\hbar$

Leucaena glauca Benth. $\hbar$

Mimosa acanthocarpa Poir. $1 /$

- glomerata Forst. $\hbar$

- pudica L. $\odot$

\section{Myrtaceae.}

Baeckea virgata Andr. $\hbar$ Callistemon pungens $\mathrm{G}$. Don. $\hbar$ - rugulosum Dec. $\hbar$

Eucalyptus botryoides Smith. $\hbar$ - resinifera Smith. $\hbar$

Leptospermum myrtifolium Sieb. $\hbar$

Melaleuca cuticularis Labil. $\hbar$

- hypericifolia Smith. $\hbar$

Myrtus communis $\mathrm{L}$. $\hbar$

Tristania conferta R. Br. $\hbar$

- laurina $\mathrm{R} . \mathrm{Br}$. $\hbar$

\section{Nyctaginaceae.}

Allionia nyctaginea Michx. 2f Mirabilis dichotoma L. $\odot$

- Jalapa I.. $\odot$

- longiflora L. $\odot$

\section{Nymphaeaceae.}

Nuphar luteum L. 요

Nymphaea alba L. 2)

- coerulea Savign.

\section{Ochnaceae.}

Ochna multiflora Dec. $\hbar$

\section{Onagraceae.}

Clarkia elegans Dougl. $\odot$

Epilobium angustifolium L. 9f

- Dodonaei Vill. 24

Fuchsia arborescens Sims. $\hbar$

- corymbiflora R. P. $\hbar$

- microphylla H. B. $\hbar$

- procumbens R. Cunn. $\hbar$

Gaura parviflora Hook. $\odot$ 
Godetia amoena var. Lindleyana Spch. $\odot$

Jussiaea Blumeana Dec. ㅇ

- salicifolia H. B. 29

Lopezia coronata Andr. $\odot$

- racemosa Cav. $\odot$

Oenothera amoena Lchm. $\odot$

- Fraseri Pursh. 2)

- fruticosa L. ?f

- glauca Michx. 24

- grandiflora Lam..

- odorata Jacq. 24

- pusilla Michx. ข|

- purpurea Curt. $\odot$

\section{Orobanchaceae.}

Orobanche ramosa L. 24

\section{Oxalidaceae}

Biophytum proliferum DC. 24

- sensitivum DC. 24

Oxalis corniculata L. var. fol. atropurpureis L. $\odot$

- stricta L. 2

- valdiviensis Barn.

\section{Papaveraceae.}

Argemone mexicana L. $\odot$

Bocconia microcarpa May. $\odot$

Chelidonium majus L. ㅇ

- $\quad$ - var. laciniata L. 2f

Corydalis ophiocarpa Hook. et Thoms. 2f

Eschscholtzia californica Chmss. $\odot$

Glaucium corniculatum v. tricolor

Curt. $\odot$

Ilunnemannia fumariaefolia Sweet. 2f

Macleya cordata $\mathrm{R}$. Br. ?ै

Meconopsis cambrica Vign. 2)

Papaver alpinum L. 2f

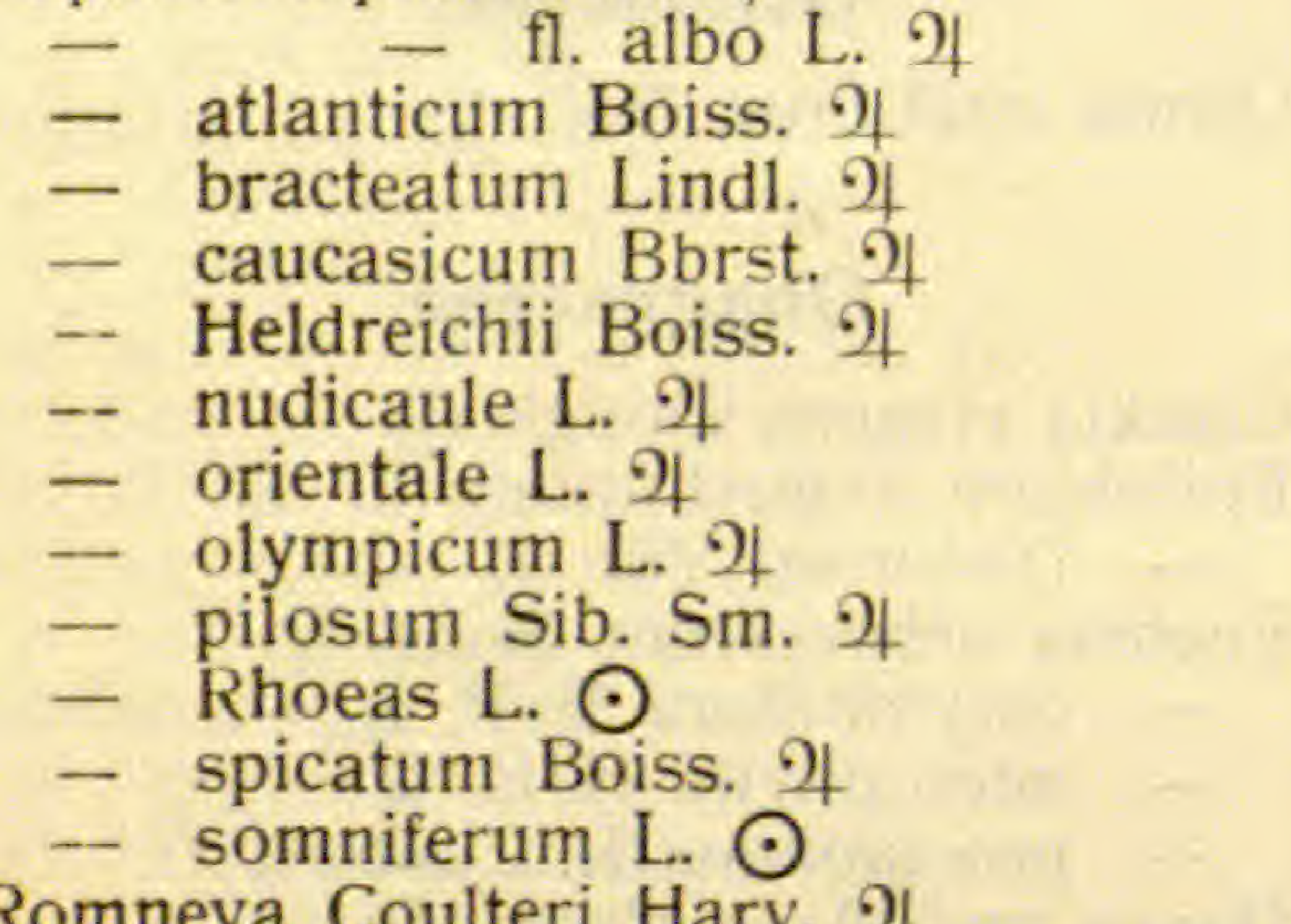

Romneya Coulteri Harv. of

\section{Papilionaceae.}

Abrus precatorius L. 24

Anisolotus Wrangeliana Bernh. $\odot$

Astragalus boeticus L. $\odot$

- Cicer L. 24

- galegiformis L. ?

- ponticus Pall. 2)

- stipulatus Don. 2f

Baptisia australis R. Br. 24.

- minor Lehm. 24

Callistachys lanceolata Vent. $\hbar$

Caragana Altagana Poir. $\hbar$

- arborescens Lam. $\hbar$

Cicer arietinum L. $\odot$

Clitoria ternata L. भ)

Coronilla scorpioides Dec. $\odot$

Cytisus Laburnum L. $\hbar$

- Linkii Janka. $\hbar$

- vulgaris Griseb. $\hbar$

Desmodium canadense DC. $\hbar$

Dolichos Catiang L. $\odot$

- Lubia Forsk. $\odot$

Dorycnium latifolium Willd. $\hbar$

Ervum Ervilia L. $\odot$

- monanthos L. $\odot$

Galega officinalis L. 2)

- _ fl. albo L. भ|

- orientalis Lam. 9

Genista elatior Koch. थ|

- radiata Scop. 24

- scoparia Lam. Of

- thyrsiflora Booth. $\hbar$

- tinctoria L. $\hbar$

- - var. virgata W. $\hbar$

Glycyrrhiza echinata L. 2)

- foetida Desf.

- lepidota Pursh. थf

Hedysarum grandiflorum Pall. थf

- obscurum L. 2)

- sibiricum Poir. 24

Indigofera argentea $\mathrm{L}$. $\hbar_{\mathrm{t}}$

- Dosua Hamilt. $\hbar$

- pulchella hort. $\hbar$

- tinctoria Lin. $\hbar$

Lathyrus Aphaca L. $\odot$

- articulatus L. $\odot$

- Clymenum L. $\odot$

- heterophyllos L. 24

- latifolius L. 2)

- mexicanus Wender. $\odot$

- Ochrus Dec. $\odot$

- odoratus L. $\odot$

- pseudaphaca L. $\odot$

- tuberosus L. 2)

- vernus Bernh. 2f

Lespedezia bicolor Turez. $\hbar_{\imath}$

Lotus biflorus Ser. $\odot$

- ornithopodioides L. $\odot$

- Tetragonolobus L. $\odot$ 
Lotus uliginosus L. ข

Lupinus albus L. $\odot$

- Barkeri Lindl. $\odot$

- Cruikshanksii Hook. $\odot$

- Douglasii Agardh. $\odot$

- Hartwegii Lindl. $\odot$

- perennis L. ?।

- polyphyllus Lindl. 2

- succulentus Doug1. $\odot$

Medicago falcata Lin. ?)

- glutinosa Bbrst. 24

- marginata Willd. $\odot$

- scutellata Lam. $\odot$

Melilotus coeruleus Desr. $\odot$

- albus Desr. -)

- macrorrhiza Pers. 24

- officinalis Pers. ..

Onobrychis crista galli Lam. $\odot$

- montana DC. 91

- sativa Lam. 2

Ononis spinosa L. 24.

- - fl. albo L. 2

Orobus laevigatus W. K. 2

- luteus L. 9

- niger L. 2)

- pallescens Bbrst. 24

- vernus L. 2/

Phaseolus capensis L. $\odot$

- compressus Dec. $\odot$

- multiflorus Lam. $\odot$

- radiatus $\mathrm{L}$. $\odot$

- tuberosus Lour. 9

- vulgaris L. $\odot$

- - var. Zebrina L. $\odot$

Pocockia cretica Dec. $\odot$

Rhynchosia densiflora Dec. $\hbar$

Scorpiurus muricata L. $\odot$ subvillosa L. 2

Securigera Coronilla Dec. $\odot$

Soja hispida Moench. $\odot$

Sophora flavescens Ait. ?|

Spartium junceum Link. $\hbar$

Swainsonia coronillifolia Salisb. $\hbar$ - galegifolia R. Br. $\hbar$

Tephrosia villosa Pers. थ

Thermopsis fabacea D. C. 2

Trifolium alpestre L. 2)

- elegans Sav. 24

- fragiferum L. 24

- incarnatum L. 2

- pannonicum Jacq. 9

- procumbens L. $\odot$

- rubens L. 2

Trigonella calliceras Fisch. $\odot$

- corniculata L. $\odot$

- Foenum graecum Lin. $\odot$

Vicia angustifolia Roth. $\odot$

- cassubica L. 2

- Cracca L. 2

- dumetorum L. 2
Vicia Faba L. $\odot$

- ferruginea Besser. $\odot$

- Gerardii D. C. 24

- microphylla D'Urvil. 24

- Orobus Dec. 2

- pisiformis L. 2

- sativa L. $\odot$

Vigna glabra Savi. $\odot$

\section{Passifloraceae.}

Passiflora coccinea Aubl. 와

- coerulea L. 2)

- gracilis Link. 2

Pedaliaceae.

Martynia fragrans Lindl. $\odot$

- proboscidea Glox. $\odot$

- lutea L. $\odot$

\section{Phytolaccaceae.}

Phytolacca decandra L. 24 - Kaempheri A. Gray. 의 Rivina aurantiaca Wood. 9

- brasiliensis Nocca. 24

- humilis L. $\hbar$

\section{Piperaceae.}

Peperomia maculosa Hook. 24 - pellucida H. B. $\odot$

\section{Pittosporaceae.}

Pittosporum Tobira Ait. $\hbar$

\section{Plantaginaceae.}

Plantago alpina L. ?)

- amplexicaulis Cav. 24

- arenaria W. K. 24

- gentianoides Sm. 24

- Lagopus L. 2f

- lanceolata L. 2

- major L. 2

- major var. purpurascens L. 24

- maritima L. 24

- media L. 24

- Psyllium L. $\odot$

- subulata L. 21.

\section{Plumbaginaceae.}

Armeria Welwitschii Boiss. 24 Goniolimon tataricum Boiss. 24 Statice virgata Willd. 4 


\section{Polemoniaceae.}

Cobaea scandens Cav. थ

Phlox decussata L. ?

- rosea hort. 2)

- suaveolens Ait. 2)

Polemonium coeruleum L. ?

- panciflorum Watz. 2।

- Richardsonii Grah. 24

\section{Polygonaceae.}

Atraphaxis lanceolata DC. $\odot$

Mühlenbeckia complexa Meissn. 와

Polygonum baldschuanicum Regl. 2 4

- emarginatum Roth. 2)

- orientale L. $\odot$

- sachalinense F. Schmidt. 24

- virginianum L. 24

- viviparum L. 2

Rheum officinale Bail. ?

- Rhaponticum L. 9

- undulatum L. 2

Rumex crispus L. ข)

- Patientia L. 와

- purpureus Poir. 24

- salicifolius Weinm. ㄱ

- scutatus L. 2

\section{Portulacaceae.}

Calandrinia Menziesii Hook, $\odot$

- sericea Hook. 2

- umbellata DC. $\odot$

Claytonia perfoliata Don. $\odot$

Portulaca oleracea L. $\odot$

- pilosa L. $\odot$

Talinum patens Willd. $\odot$

\section{Primulaceae.}

Androsace elongata L. $\odot$

- lactea Vill. $\odot$

- lactiflora Fisch. 2

- maxima L. $\odot$

- septentrionalis L. $\odot$

Dodecatheon grandiflorum Moore. 24

- Jeffreyi Moore. 2

Lysimachia ciliata Lin. 와

- punctata Lin. 2|

- quadrifolia L. 29

- thyrsiflora L. 2f

- verticillata Bbrst. \%

Primula Auricula L. 2)

- japonica A. Gray. 2

- officinalis Jacq. 24

- rosea Royle. 2f

\section{Ranunculaceae.}

Aconitum Anthora L. $\mathcal{4}$
Aconitum Lycoctonum L. ข)

- Napellus L. 2f

- septentrionale Mart. \%

Actaea palmata Dec. 24

- spicata L. 24

Adonis aestivalis Lin. $\odot$

Anemone alpina Lin. ?)

- coronaria Lin. 2f

- fulgens Gay. 24

- multifida Poir. 24

- narcissiflora Lin. 2y

- palmata L. 24

- sylvestris L. 29

- virginiana Lin. 24

Aquilegia coerulea James. थ|

- glandulosa Fisch 2

- Skinneri Hook. 9y

- vulgaris L. 24

Caltha palustris Lin.

Cimicifuga foetida L. 와

Clematis angustifolia Jacq. 24

- gebleriana Bong. 24

- integrifolia L. 24

- flammula L. 24

- Viorna L. $\hbar$

- viticella L. 24

Delphinium Ajacis L. $\odot$

- cashmerianum Royle. 24

- cheilanthum Fisch. 21

- elatum L. 2)

- exaltatum Ait. 21

- grandiflorum L 24

- intermedium Ait. 2

- Maackianum Kgl. 24

- montanum L.24

- palmatifidum Dec. 24

- triste Fisch. 24

- Woehlerii Lam. 2)

Nigella damascena L. $\odot$

- orientalis L. $\odot$

- sativa L. $\odot$

Paeonia corallina Retz. 2f

- decora Anders. var. elatiot Hort. 2)

- officinalis L. 2)

- $\quad$ - fl. pl. Pall. 2

- paradoxa Anders. 2

- sinensis Pall. 24

- - var. rosea hort. 24

Pulsatilla Halleri Willd. of

Ranunculus arvensis L. $\odot$

- cassubicus L. 24

- muricatus L. $\odot$

- repens L. 24

- serbicus Vis. 21

Thalictrum aquilegifolium L. 24

- cinereum Desf. 24

- Cornuti L. 2)

- elatum Jacq. 24

- foetidum L. 29 
Thalictrum glaucum Desf. 2

- minus L. 2

- purpurascens L. 24

- sylvaticum Koch. 24

- tuberosum L. 24

Trollius Altaicus C. A. Mey. 2)

- asiaticus L. 2

- europaeus L. 94

\section{Resedaceae.}

Astrocarpus sesamoides DC. $\odot$

Reseda abyssinica Fresen.

- alba L. $\odot$

- glauca L. 9

- luteola L. $\odot$

- myriophylla Tenor. 24

- odorata L. $\odot$

\section{Rhamnaceae.}

Colletia spinosa Lam. $\hbar$ Pomaderris elliptica Labil. $\hbar$ Rhamnus buxifolia Poir. $\hbar$

\section{Rosaceae.}

Agrimonia dahurica Willd. 24

- Eupatorium Lin. 24

- odorata Mill. 2|

- leucantha Kunze. 2

- repens L. 24

- suaveolens Pursh. 94

Alchemilla fissa Günth. 24

$$
\text { - vulgaris L. 24 }
$$

Cotoneaster horizontalis L. $\hbar$

Dryas octopetala Lin. 이

- lanata Kern. 2

Geum carolinianum Walt. 2

- coccineum Sm. 24

- japonicum Thbg. 9

- ranunculoides Serring. 2

- rivale L. 24

- Rossii Ser.

- strictum Ait. 2

Mespilus germanica L. $\hbar$

Potentilla alpestris Hall. 24

- atrosanguinea Don. 24

- collina Wibel. 94

- inclinata Vill. 24

- palustris L. 24

- rupestris L. 21

Rhodotypus kerriodes S. et $Z$. $\hbar$

Rosa gallica L. $\hbar$

- laxa Retz. $\hbar$

- pimpinellifolia Lin. $\hbar$

- turbinata Ait. $\hbar$

Sanguisorba canadensis. L. ㅇ

- mauritanica Desf. 24

- officinalis L. 24

- tenuifolia Fisch. 24
Sibbaldia procumbens L. 이

Spiraea Filipendula L. .)

- lobata hort. 2

\section{Rubiaceae.}

Asperula arvensis L. श)

- galioides M. B. 2)

- humifusa Bess. 2)

- odorata L. 2)

- tinctoria L. 24

Cruciannella aegyptiaca L. $\odot$

- angustifolia L. $\odot$

- gilanica Trin. 2)

Galium boreale L. 2)

- Mollugo L. 2)

- purpureum L. ?|

Richardsonia scabra L. -)

Sherardia arvensis L. ?/

Vaillantia hispida L. $\odot$

- muralis L. $\odot$

\section{Rutaceae.}

Dictamnus albus L. थै

- albus var. ruber. L. \%

- Fraxinella Pers. 9

Melicope ternata Forst. $\hbar$

Ptelea trifoliata L. $\hbar$

Ruta graveolens L. थ|

- macrophylla Soland. 9)

- montana Mill. 2)

\section{Sapindaceae.}

Koelreuteria paniculata Laxm. $\hbar$. Sapindus indica Poir. $\hbar$

\section{Sapotaceae.}

Achras Sapota L. $\hbar$

Bumelia tenax Willd. $\hbar$

\section{Saxifragaceae.}

Francoa appendiculata Cav. 2) Heuchera alba A. Gray. 24

- americana L. 2)

- cylindracea Lindl. 9)

- hispida Pursh. 2

- micrantha Dougl. 2|

- Richardsoni R. Br. 2

- sanguinea A. Gray. 24

Parnassia palustris L. ?]

Saxifraga Aizoon L. ?

- Cotyledon L. 24

- cartilaginea Willd. 2

- caespitosa L. 2)

- ceratophylla Ait. 2)

- cordifolia L. 2

- crassifolia L. 2y 
Saxifraga Cymbalaria L. 의

- decipiens L. \%)

- granulata L. 2)

- longifolia Lapeyr. 24

- rodundifolia $\mathrm{L}$. 24

- tenella Wulf. 2)

- umbrosa L. 9|

- - fol. var. L. 2)

Tellina grandiflora Lind. 24

Tiarella cordifolia L. 와

\section{Scrophulariaceae.}

Anthirrhinum calycinum Vent. $\odot$ - majus L. 24

- Orontium L. $\odot$

Calceolaria scabiosifolia Sms. $\odot$

Collinsia bicolor Benth. $\odot$

Digitalis ambigua Murr. -)

- ferruginea L. $\odot$

- gloxiniiflora L. $\odot$

- - var. purpurea L. $\odot$

- purpurea L. var. alba. $\odot$

- grandiflora Lam. 24

- lanata Ehrh. 9

- lutea L. 24

- purpurea L. 29

- viridiflora Lindl. ข

Erinus alpinus L. 아.

Gratiola officinalis Lin. 24

Linaria heterophylla Desf.

- minor Desf. $\odot$

- lusitanica Spr. ? triphylla Willd. $\odot$

Maurandia scandens Gray. $\odot$

Mimulus cardinalis Lin. $\odot$

- hybridus hort. 24

- lutens L. 24

Nemesia floribunda Lehm. $\odot$

Pentastemon barbatus Nutt. 24

- confertus Dougl. 24

- diffusus Dougl. 2)

- glaber Pursh. 2

- glaucus Grah. \%)

- gracilis Nutt. 2)

- laevigatus Ait. 9f

- pubescens Ait. -)

Scrophularia nodosa L. 2)

- vernalis L. 24

Torenia exapendiculata Rgl. $\odot$ - parviflora Wall. 24

Verbascum Blattaria L. $\odot$

- Kanitzianum Link. $\odot$

- Lychnitis L. $\odot$

- nigrum L. 9f

- orientale Bbrst. $\odot$

- phlomoides L. $\odot$

- thapsiforme Schrad. $\odot$

- Thapsus L. 2)

Veronica gentianodes Vahl. 24
Veronica incana L. 2)

- longifolia L. \%

- $\quad$ - fl. albo L. 9|

- mollis Willd y

- officinalis L. ㅇ

- pectinata L. 2f

- polystachya Link. -)

- sibirica L. 9

- spicata L. 2)

- spuria L. 9l

- urticifolia L. .

- virginica L. 2)

\section{Solanaceae.}

Atropa Belladonna L. 2)

Capsicum annuum Lin. $(-$

- longum Dec. $\odot$

Datura Stramonium L. $\odot$

- Tatula L. $\odot$

Habrothamnus elegans Scheidw. $\hbar$

Hyoscyamus albus L. $\odot$

- niger L. $\odot$

Lycopersicum cerasiforme var. rubra Don. $\odot$

- Humboltii Mill. $\odot$

- piriforme var. rubra Voss.

Mandragora officinalis Bertol. ?)

Nicandra physaloides Gaert. $\odot$

Nicotiana affinis Moore. $\odot$

- Langsdorffi Schrad. $\odot$

- rustica L. $\odot$

- Tabacum L. $\odot$

Petunia nyctaginiflora Juss. $\odot$

Solanum Balbisii Dum. $\odot$

- Dulcamara L. $\hbar$

- Gilo Requin. $\odot$

- gracile Ott. 2)

- marginatum L. $\hbar$

- melongena L. $\odot$

- miniatum Bernh.

- nigrum L. $\odot$

- Pseudo - Capsicum L. $\hbar$

- villosum Linn. $\odot$

\section{Staphyleaceae.}

Staphylea trifoliata L. $\hbar$

\section{Thymelaeaceae.}

Daphne Laureola Lin. $\hbar$

- Mezereum Lin. $\hbar$

- - fl. albo Lin. $\hbar$

Pimelea ligustrina Labill. $\hbar$

\section{Umbelliferae.}

Anethum graveolens L. $\odot$

Archangelica officinalis Hoffm. $\odot$ 
Astrantia Biebersteinii Fisch. et Mey. 구

- carniolica Jacq. 2)

- helleborifolia Salisb. 2

- major L. of

Athamantha Mathiola Wulf. Jacq. 21 Bupleurum falcatum L. ?f

- graminifolium Wahl. ข

- longifolium L. 아

- multinerve Dec. ข|

- rotundifolium Link. $\odot$

Bifora testiculata D. C. $\odot$

Carum Bulbocastanum Koch. 2)

- Carvi L. $\odot$

Chaerophyllum aromaticum L. 2)

- aureum L. 2)

- bulbosum Lin. $\odot$

- sativum Lam. ?

Cicuta virosa L. ?

Conium maculatum Lin. of

Coriandrum sativum L. $\odot$

Cryptotaenia canadensis Dec. 가

Eryngium agavifolium Griseb. ?|

- amethystinum L. 가

- angustifolium L. 2

- bromelifolium Laroche. 29

- Bourgatii Gou. ?

- coeruleum Bieb. y

- dichotomum Desf. 9/

- giganteum M. B. 와

- oliverianum Delar. 2f

-. planum L. 2)

- rigidum Lam. 2f

Ferula Ferulago Lin. 9|

Foeniculum vulgare Gaert. (-)

Heracleum Lehmannianum Rgl. $y$

- Sphondylium L. 24

Hydrocotyle moschata Forst. 의

- rotundifolia Roxb. y

- vulgaris L. 2)

Imperatoria Ostruthium L. 의

Laserpitium intermedium L. ๆ)

Levisticum officinalis Koch. 2)

- paludapifolium Lam. 24

Lophosciadium millefolium Dec. ข

Meum athamanticum Jacq. 2|

Myrrhis odorata Scop.

Oenanthe Lachenalii Gmel. ㅇ

- fistulosa. 9)

- peucedanifolia Pollich. 2f

- pimpinelloides L. 9f

Pachypleurum alpinum Ledeb. ?|

Pastinaca sativa L. 2)

Petroselinum sativum Hoffm.

Peucedanum sylvestre Dec. 24

Pimpinella Anisum L. $\odot$

- magna L. 2]

- rotundifolia Bieb. 2/

- Saxifraga L. 29

Prangos thapsoides Dec. 9|

Sanicula europaea L. ?|
Selinum Carvifolium L. 2)

Seseli libanotis C. Koch. 29

- tenuifolium Ledeb. 2

Siler aquilegifolium Gaertn of

Sium angustifolium Koch. 2)

- latifolium L. ?

Trinia Hoffmani Bbrst.

- Kitaibelii Bbrst. $\odot$

Zizia aurea Koch 와

- integerrima Dec. 94

\section{Urticaceae.}

Boehmeria nivea Gaudich. 2f

Dorstenia arifolia Lam. ?

- Houstoni. L. 2)

Forskohlea angustifolia Retz. $\odot$

Parietaria lusitanica. L. $\odot$

Pilea muscosa Lind1. ข]

Procris australis Spr. 2)

Urtica balearica L. $\odot$

- cannabina L. $\odot$

- dioica L. 2]

- Gigas Cunningh. $\hbar$

- macrophylla Thunbg. ๑)

- morides Cunningh. $\hbar$

- palmata Forsk. 2)

- piluliferea L. $\odot$

\section{Valerianaceae.}

Centranthus Calcitrapa Dufresn. $\odot$

- macrosiphon Boiss. $\odot$

- ruber D. C. 2

Valeriana officinalis L. 2)

- sambucifolia Mik. 2

Valerianella pumila Dec. $\odot$

\section{Verbenaceae.}

Duranta Ellisia Jacq. $\odot$

Verbena chamaedryfolia Juss. $\odot$

- elegans H. B. 2)

- officinalis L. 9)

- paniculata L. 9)

- urticifolia L. .

- venosa Hook. 24

\section{Violaceae.}

Viola cornuta L. 2)

- elatior Fries. 9)

- mirabilis L. 9f

- pinnata L. 2)

- primulifolia L. 2)

- tricolor L. $\odot$

\section{Zygophyllaceae.}

Peganum Harmala L. 2)

Zygophyllum Fabago L. ?| 


\section{Anhang.}

Pflanzen können abgegeben werden von folgenden Sedum- und

\section{Sempervivum-Arten :}

Sedum acre L. ㅇ

- Aizon L. 9

- album L. P|

- boloniense Loisel. If

- calabricum Ten. 2)

- crenatum Rad. 24

- cruciatum Desf. 24

- cyaneum Rad. 2

- elegans Lejeun. 24

- Ewersii Lood. 24

- ibericum Stev. 2)

- Kamtschaticum Regel. 2)

- Maximowiczii Regel. ')

- purpurascens Link. -)

- reflexum Willd. ?

- Rhodiola Dec. ?

- roseum Stev. ?|

- sexangulare L. ?)

- speciosum hort. -)

- spurium Bbrst.

- Telephium L. 2)

- Wallichianum Hook. ขf

Sempervivum albidum Lehm. et Schnittsp. थ)

- acuminatum Schott. ?|

- arachnoideum L. -

- arenarium Koch. 2)

- arvernense Schott. 2)

- assimile Schott. 9)

- blandum Schott. ?)

- Boutignyanum Lk. et Sch. 24

- Braunii Funk. 24.

- californicum hort. 2)

- canescens R. S. - )

- Comollii Rota. ?

- cornutum Schott. 9)

- debile Schott. - $)$

- Delastrii Lehm. et Schnittsp. 2

- dolomiticum Hausm. 9f

- fimbriatum Lehm. etSchnittsp. 2
Sempervivum flagelliforme Fisch. f

- Funkii A. Br. 2)

- - brachypetalum $\mathrm{A} . \mathrm{Br}$. 9)

- glaucum Ten. 9]

- globiferum L. .)

- grandiflorum Haw. 9|

- Hausmannii Schott. ?

- heterotrichum Schott. 9f

- Heuffelii Schott. ?

- hirtum L. 9f

- hispidulum Schott. 2)

- Laggeri Schott. ')

- Mettenianum Lehm. -)

- modestum Jord. ?

- montanum L. ?)

- patens Grieseb. 2)

- pennicillatum Schott 9t

- piliferum hort. -)

- populifolium L. 2)

- purpureum L. ')

- Reginae hort. 9y

- Requienii R. S. 9|

- robustum Himp. 9

- rubicundum Schur. 29

- ruthenicum hort. ㅇ

- Schlehani Schott 9

- Schottii Br. 2f

- Schnittspahnii Dill. 2)

- soboliferum Sims. 2)

- spinulifolium hort. 24.

- stenopetalum Lehm. et. Schnittsp. 의

- tectorum L. .

- tomentosum Lehm. 2)

- triste hort. 2)

- Verloti Jord. 24

- - Borserii Jord. ?f

- - glaucum Jord. थ

- violaceum Blasius. y

- Widderi L. 24.

- Wulfenii Hopp. 94

Bestellungen werden bis zum 15. Februar erbeten.

Prof. Dr. M. Möbius

Direktor.
Rudolf Günther

Obergärtner. 


\section{SÄMEREIEN}

zum Tausch angeboten

vom

\section{Botanischen Garten in Frankfurt am Main.}

\section{Pteridophyta.}

\section{Filices.}

Adiantum crenatum Fisch.

- - v. gracillimum Moench.

- Edgeworthii Hook.

- gracillimum v. Houtte.

- hispidulum Sw.

- peruvianum Klotsch.

- trapeziforme L.

Allosorus rotundifolia $\mathrm{Kz}$.

Aneimia laciniata Lk.

- Phyllitidis Sw.

Aspidium coriaceum Sm.

- Filix mas monstrosum grandiceps.

- trifoliatum Sw.

- violascens Lk.

Asplenium adiantum nigrum L.

- Halleri Spreng.

- furcatum L.

- septentrionale Hoffm.

- Trichomanes L.

Blechnum brasiliense Desf.

- Gibbum Labill.

- longifolium H. B.

- occidentale L.

Ceratopteris thalictroides L, $\odot$

Chrysodium cuspidatum Kuhn.

Davallia bullata Wahl.

- dissecta L.

- gibberosa Moore.

Dennstaedtia tenera Moore.

Fadyenia prolifera Hook.

Gymnogramme palmata Lk.

Lygodium japonicum Sw.

Nephrolepis tuberosa Presl.

Platycerium alcicorne Desf.

- grande Sm.
Platycerium Willinckii Moore.

Pteris cretica L.

- - v. albo-lineata May.

- longifolia L.

- palmata Willd.

- serrulata L. v. cristata Veitch.

Woodwardia caudata Cav.

- radicans Sw.

\section{Marsiliaceae.}

Marsilia hirsuta R. Br. 24

- quadrifoliata L. 24

\section{Selaginellaceae.}

Selaginella Braunii Back. 2)

- caulescens Spr. 24

- cuspidata Spr. 24

\section{Monocotyledoneae.}

\section{Alismaceae.}

Alisma Plantago L. 2)

Butomus umbellatus L. 9

Sagittaria montevidensis Chmss. et Schlecht. 9

\section{Amaryllidaceae.}

Cooperia Drummondi Hook. 9f Haemanthus Nelsonii Jacq. 24 - tigrinus Jacq. of

Zephyranthes Atamasco Herb. 94 


\section{Araceae.}

Anthurium Scherzerianum Schott. 94 Arum italicum Mill. ?)

Calla palustris L. ?)

\section{Bromeliaceae.}

Billbergia zebrina Lindl. 2)

Caraguata linguata L. 2)

Dyckia remotiflora Otto Dietr. 24

- sulphurea Lem. - 4

- - v. Houttei Lem. 9f

Encholirion corallinum Linden. 9)

Guzmannia tricolor R. P. 2)

Pitcairnia odorata Ang. 의

\section{Commelinaceae.}

Commelina coelestis Willd. ?|

Tinnantia erecta. Schlecht. $\odot$

\section{Cyperaceae.}

Carex cyperoides L. ๆ

- Grayana Dew. 94

- japonica fol. var.

- pendula Moench.

- stricta Good. ?|

- xanthophysa Wahlbg. 2f

Cyperus laxus R. Br. थ|

- longus L. 2)

- natalensis L. 29

- Papyrus L. 2)

Scirpus lacustris. V. Zebrina L. 2)

- radicans Schkr. 24

- sylvaticus L. 2)

\section{Gramineae.}

Alopecurus arundinaceus Poir. थ/ Andropogon Ischaemum L. 2)

Avena fatua L. $\odot$

- orientalis var. flava Kck. $\odot$

- strigosa Schreb. $\odot$

Brizopyrum distachyum R. S. $\odot$ - siculum Link. 9]

Bromus arvensis L. $\odot$

- macrostachys Poir. var. lanuginosa Desf. $\odot$

- maximus Desf. $\odot$

- pendulinus Schrad. $\odot$

Calamagrostis Epigaios L. 94

Coix Lacryma L. $\odot$

Corynephorus canescens Bernh. 94

Cynosurus echinatus L. $\odot$

Dactylis glomerata L. थ
Festuca gigantea Vill. $\odot$

- glauca Lain. ?)

- ovina L. ?

- pectinella Delil. $\odot$

Hierochloe borealis R. S. थf

Hordeum distichum var. nutans L. $\odot$

- hexastichum var. pyramidata Kke. $\odot$

- maritimum With $\odot$

- zeocriton L. $\odot$

Kneleria cristata Vahl. 2)

Lagurus ovatus L. $\odot$

Lasiagrostis Calamagrostis Link. \%)

Melica altissima L. 2)

- exasperata R. S. 9)

Molinia coerulea Moench. ?)

Oryza sativa var. mutica L. $\odot$

Panicum capillare L. $\odot$

- miliaceum var. alba L. $\odot$

- sanguinale Scop. $\odot$

Pennicillaria spicata Willd. $\odot$

Pennisetum compressum R. Br, ?)

- longistylum Hochst.

- Rüppellii Steud.

- villosum R. Br.

Phalaris canariensis L. $\odot$

Phleum asperum Vill. $\odot$

Poa alpina L. 94

Setaria ambigua Good. $\odot$

- italica var. aurantiaca Kke. $\odot$

Sorghum sacharatum Pers. $\odot$

- vulgare Pers. $\odot$

Stipa capillata L. 9

- gigantea Lagasc. 9f

- splendens Trin. 29

Triticum ciliatum Dec. $\odot$

- durum v. affinis Kke. $\odot$

- monococcum L, $\odot$

- polonicum var. attenuata L. $\odot$

- Spelta L. $\odot$

- - var. alba L. $\odot$

Uniola latifolia L. 2)

Zea Mais L. $\odot$

- - americana Mill. ()

- - var. rubra Bonef. $\odot$

- - - violacea Kk. $\odot$

- - japonica fol. var.

\section{Iridaceae.}

Anomatheca cruenta Lindl. of

Iris cuprea Pursh. 9f

- mauritanica Spr. 9

- lutescens Lam. 9|

- pseudacorus L. 9)

- sibirica L. 94

- spuria L. 9f

Libertia formosa Grah 2 
Sisyrinchium anceps Lem. 의 - Bermudianum L. 9) Sparaxis grandiflora Ker. - 4

\section{Juncaceae.}

Juncus squarrosus L. 9f

Luzula nivea Desv. 94

\section{Juncaginaceae.}

Triglochin maritimus L. .)

\section{Liliaceae.}

Albuca fastigiata Dryand. 2)

Allium fistulosum L. 의

- fragrans Vent. 9]

- globosum M. B. 9)

- nigrum L. 2f

- Porrum L. 9)

- rotundum L. 9

- strictum Schrad. 24

- ursinum L. 9y

- Victorialis L. 9f

Anthericum Liliago L. \%)

Asparagus officinalis L. 9)

- Sprengeri Rgl. of

Asphodelus albus Willd. ?f

Bowiea volubilis Haworth. 9)

Eremurus caucasicus Sweet. ')

Eucomis punctata Ait. 2)

- regia Ait. ?t

Fritillaria imperialis L. 9)

- Meleagris L. ㅇ

- pyrenaica L. 9

Funkia ovata Spr. 의

- Sieboldiana Lood. 91

Hemerocallis flava L. ?)

Hyacinthus candicans Bak. 의

Kniphofia aloides Moench. ?f

Lilium candidum L. ?)

- giganteum Wallich. 94

- Martagon L. ?t

- tigrinum Gawl. 9)

- sulphureum Hort. 9|

Muscari comosum Willd. of

Ornithogalum narbonense L. .)

Uropetalum serotinum Desf. $\odot$

Zygadenus glaucus Nutt. $\odot$

\section{Melanthaceae.}

Veratrum Lobelianum Bernh. 의 - nigrum L. 9)

\section{Orchidaceae.}

Gymnadenia conopea R. Br.

Sturmia Loeselii Rchbch. ㅇ․

\section{Typhaceae.}

Typha augustifolia L. 9|

- latifolia L. ?)

- Laxmanni L. 2)

- minor Smith. 29

\section{Dicotyledoneae.}

\section{Acanthaceae.}

Acanthus longifolius Poir. 9)

- mollis L. ?)

Ruellia rosea Wall. कf

- strepens L. 와

\section{Amarantaceae.}

Amarantus albus L. $\odot$

- caudatus L. $\odot$

- - fl. rubro L. $\odot$

- paniculatus L. $\odot$

- retroflexus L. $\odot$

- speciosus L. $\odot$

- var. monstrosa L. $\odot$

- tricolor L. $\odot$

Celosia cristata L. $\odot$

- - var. Thomsoni Balf. $\odot$

Gomphrena globosa L. $\odot$

\section{Anacardiaceae.}

Rhus vernicifera Dec. ข)

\section{Anonaceae.}

Anona Cherimolia Mill. of

\section{Apocynaceae.}

Amsonia angustifolia Michx. 2f

- latifolia Michx. 24

- salicifolia Pursh. 9f

- tabernaemontana Wall. 9f

Apocynum cannabinum L. 94

\section{Araliaceae.}

Aralia racemosa $I . \hbar$ 


\section{Asclepiadaceae.}

Asclepias Cornuti L. ๆ

- curassavica L. .

- Douglasii Michx. 의

- incarnata L. 2)

- syriaca L. 21

Cynanchum furcatum Rchbch.

- nigrum Moench. 2f

- Vincetoxicum L. थ|

\section{Balsaminaceae.}

Impatiens Balsamina L. $\odot$

- longicornis Wall. $\odot$

\section{Begoniaceae.}

Begonia gracilis H. B. $\odot$

- semperflorens L. $\odot$

- Schmidtii L. $\odot$

\section{Berberidaceae.}

Podophyllum Emodi Wall. ')

\section{Betulaceae.}

Betula lenta L. $\hbar$

\section{Bignoniaceae.}

Catalpa Kaempferi Sieb. et. Zucc. $\hbar$ Incarvillea Olgae Rgl. क्

\section{Borraginaceae.}

Anchusa italica Retz. ㄱ

- sempervirens Fisch. et Traut. 2|

Borrago officinalis L. $\odot$

Cerinthe alpina Schult. $\odot$

- retorta Sib. $\odot$

- minor L. $\odot$

Cynoglossum officinale L. 9)

- longiflorum Lehm. ?

Lithospermum officinale L. ?

Myosotis silvatica Hoffm. भ

Nonnea rosea Link. $\odot$

Omphalodes linifolia Moench. $\odot$

Phacelia tanacetifolia Benth.

Pulmonaria montana Wulff. भI

Symphytum asperrimum M. B. 94

- bohemicum Schmidt. 9|

- Carmosina Hort. 9f

- officinale L. 2

\section{Campanulaceae.}

Campanula alliariifolia Willd. ?

- barbata L. 9)

- bononiensis L. थ

- Cervicaria L. 2)

- grandis Fisch. et Mey. 9|

- lactiflora M. B. 2f

- lamiifolia Bbrst. 9y

- macrostyla Boiss. 9

- Medium L. $\odot$

- - fl. alba L. $\odot$

- - plena L. $\odot$

- - var. rosea L. $\odot$

- nobilis Lindl. 9

- persicifolia L. ㅇ

- rapunculoides L. 29

- rhomboidea L. 9y

- rotundifolia L. 2

- sarmentosa Ker. PI

- Scheuchzerii Vill. थ|

- strigosa Vahi. $\odot$

- Trachelium L. 9y

- Vidalii Wats.

Phyteuma orbiculare L. 9| Platycodon grandiflorum Dec. ? Specularia falcata Dec. $\odot$ Symphyandra Hoffmanni Pant.

Trachelium coeruleum L. 9)

Wahlenbergia nutabunda Dec. $\odot$

\section{Cannabinaceae.}

Cannabis sativa v. gigantea H. $\odot$ Humulus japonicus fol. var. Setz. $\odot$

- Lupulus L. $\odot$

\section{Capparidaceae.}

Cleome pungens Willd. $\odot$

\section{Caryophyllaceae.}

Agrostemma Githago L. $\odot$ - nicaeensis Willd. $\odot$ Cerastium perfoliatum L. $\odot$ Cucubalus bacciferus L. कf Dianthus alpinus L. ?f

- atrorubens All. פ|

- barbatus L. 9!

- Caryophyllus L. थ|

- Carthusianorum L. 9)

- deltoides L. 2f

- plumarius L. 2f

- prolifer L. $\odot$

- pubescens Sm. $\odot$

- Seguieri Vill.var discolorHort.24 
Dianthus superbus nanus L. ?) - sylvestris Wulf. of - serotinus W. R. थ)

Drypis spinosa L. $\hbar$

Gypsophila elegans Bbrst. $\odot$ - paniculata L. 24

Honckenya peploides. Ehrh. 와 Lychnis alpina L. ?

- Bungeana Fisch. 9|

- chalcedonica L. 9y

- viscosa L. 2)

Saponaria officinalis L. ?)

Silene alpestris Jacq. ?/

Armeria L. $\odot$

- compacta Bbrst. $\odot$

- Drumondii Hook.

- Hornemanni Steud. -9

- fimbriata Sims. 24

- fruticulosa Sims. 91

- inflata L. 2)

- nutans L. 9

- Otitis Pers. 9

- paradoxa L. थ

- pendula L. $\odot$

- quinquevulnera L. $\odot$

- syriaca Boiss. 9y

- viridiflora $\mathrm{L} . \hbar$

- Zawadskii Presl. 2)

Spergularia salina Presl. $\odot$

Telephium Imperati L. ㅇ․

Vaccaria segetalis Neck. $\odot$

\section{Chenopodiaceae.}

Acroglochin chenopodioides Schrad. $\odot$

Atriplex hortensis L. $\odot$

Blitum spicatum L. 9f

- virgatum L. $\odot$

Chenopodium album var.cymigera L.P

- bonus Henricus L. 24

- Quinoa Willd. $\odot$

Hablitzia tamnoides Bbrst. 2)

Polycnemum arvense L. $\odot$

Salsola Kali L. $\odot$

Spinacea oleracea L. $\odot$

\section{Cistaceae.}

Helianthemum guttatum Mill.

- niloticum Moench.

- rosmarinifolium Pursh.

- salicifolium Pers. $\odot$

- variabile Spach. ㅇ

\section{Compositae.}

Achillea ageratifolia L. क)

- Clavennae L. 2)
Agathaea spathulata Fisch. $\odot$

Ageratum mexicanum L. $\odot$

Alfredia stenolepis Kar. et Kir. 9)

Ammobium alatum R. Br. $\odot$

Anthemis austriaca Jacq. 아

- nobilis L. Q4

- tinctoria L. भ

- Triumphetti Dec. ข|

Arctium japonicum Hort.

Arnica montana L. ?)

Artemisia annua L. $\odot$

- Absinthium L. 24

- rupestris L. 9)

Aster alpinus L. 9f

- chinensis Bernh. $\odot$

- himalaiensis Hook. 2|

- Linosyris Bernh. 9y

Barkhausia rubra Moench. 2)

Bidens leucantha Willd. pilosa L. $\odot$

Bupthalmum salicifolium L. 의

Calais Bigelowii A. Gray. $\odot$

Calendula officinalis L. $\odot$

Calliopsis bicolor Rchbch. $\odot$

- cardaminifolia Dec. $\odot$

Carthamus tinctorius L. $\odot$

Cenia turbinata Pers. $\odot$

Centaurea alpina L. of

- americana Nutt. - 4

- axillaris Willd. 24

- calocephala Willd. 9

- Cyanus L. $\odot$

-.- glastifolia L. 의

- imperialis alba L. $\odot$

- jacea L. 2f

- Kotschyana Heuff. 21

- macrocephala Nuss. 2)

- margaritacea Tenor. 9y

- melitensis L. $\odot$

- montana alba L. 2)

- paniculata L. $\odot$

- phrygia L. 24

- pulcherrima Willd. 24

- Rhaponticum L. 24

- Scabiosa L. 21

- strobilacea Scop.

- suaveolens Willd.

- sulphurea Willd.

Chamaepeuce diacantha Dec. $\odot$

Chondrilla juncea v. latifolia L. .)

Chrysanthemum carinatum Schrad. $\odot$

- coccineum Sims. $\odot$

- corymbosum L. 24

- Balsamita L. 2y

- Leucanthemum L. 29

- macrophyllum W. et K. 와

- roseum Web. et Mohr. 24

Cichorium Intybus var. culta. L. 24 Cirsium canum All. 24

- heterophyllum All. 29 
Cnicus benedictus L. $\odot$

- pseudobenedictus Gaert. $\odot$

Coreopsis coronata Nutt. $\odot$

- tinctoria Hook. $\odot$

Cosmos sulphureus Cav. $\odot$

Crepis latifolia Ball. ?f

- maritima L. $\odot$

- pulchra L. $\odot$

- sibirica L. 24

Cryptostemma hypochondriacum R. Br. $\odot$

Cyclachaena xanthiifolia Fres. $\odot$

Cynara Cardunculus L. (-)

Dahlia coccinea Cav. of

- glabrata Lindl. 2f

- variabilis Desf. 2)

Dimorphotheca pluvialis Moench.

Doronicum caucasicum Bbrst. -2)

- macrophyllum Fisch. -y

- Pardalianches L. .)

Echenais carolinoides Cass. $\hbar$

Echinops dahurica Fisch. 이

Emilia sonchifolia Dec. $\odot$

Endoptera Dioscoridis Dec.

Erigeron speciosus Dec. oy

Eupatorium ageratifolium Dec. 의

- cannabinum L. \%)

- purpureum L. h

Filago minima Pers. $\odot$

Gaillardia aristata Pursh, 2)

- Drummondii Dec. of

- - v. grandiflora Dec. 2)

- pulchella Fouger. 2y

Geropogon glaber L. $\odot$

Gnaphalium luteum album L. $\odot$

- purpureum L. 9)

Hedypnois persica Bbrst. $\odot$

Helianthus annuus L. $\odot$

- - fl. pleno L. $\odot$

- maximilianus Schrad. $\odot$

Helichrysum bracteatum Willd. $\odot$

- elegans Don. 24

Helminthia echioides Gaert. $\odot$

Hieracium alpinum L. 아

- aurantiacum L. of

- Auricula L. 2)

- amplexicaule L. 2)

- dendatum Hoppe. จ

- eriophorum Link. \%

- Lactaris Bert. of

- lycopsifolium Froel. ?

- pulmonarioides Vill. 의

- ramosum Suter. 9)

- sabaudum L. 9y

- sylvestre Fries. 29

- villosum Link. 9

Humea elegans Sm. $\odot$

Hypochoeris glabra L. $\odot$

Inula Helenium L. 2)

- hirta L. 24
Jurinea cyanoides Dec. 요

Kentrophyllum lanatum Dec. $\odot$

- tauricum Fisch. et Meyer.

Lactuca perennis L. $\odot$

- Scariola L. 9y

- virosa L. 9

Leontopodium alpinum L. 구

- himalayanum Dec. 아

Leuzea Cynaroides Dec. $\hbar$

- conifera Dec. $\hbar$

Liatris pycnostachya Mchx.

Ligularia macrophylla Dec. ?)

- sibirica Cass. 기

- speciosa Fisch. et M. 2)

Madaria elegans Dec. $\odot$

Madia sativa Molin.

Maruta Cotula Cass. $\odot$

- lithuanica Bess. $\odot$

Matricaria Chamomilla L. $\odot$

- eximia L. $\odot$

Mulgedium sibiricum Less. 2)

Onopordon Acanthium L. $\odot$

- elongatum L. $\hbar$.

- macracanthum Clarke. $\odot$

Picris dahurica Pursh. 2)

Prenanthes purpurea L. 9f

Ptarmica alpina L. of

Pulicaria odorata Rchb. 2f

Pyrethrum corymbosum Willd. -)

- eximium hort. $\odot$

- roseum Bbrst. ?

- macrophyllum Willd. - $)$

- Parthenium Sm. 2f

- Tschihatchewii Voss. 9f

Rhagadiolus caudatus L. $\odot$

- stellatus Gaert. $\odot$

Rhaponticum scariosum Lam. 9)

Rudbeckia amplexicaulis L. 2)

- digitata Mill. 2/

- laciniata L. 9

- moschata Lood. 9f

- Neumannii Loudon. 9|

- speciosa Wender, 21.

Sanvitalia procumbens Lam. $\odot$

Scolymus maculatus L. $\odot$

Scorzonera villosa Scop. 2y

Senecio coriaceus Ait. 2)

- crispus var, rivularis Dec. y

- Doria L. 2)

- Fuschsii Gmel. 24

- nemorensis Jacq. ?f

Seriola aethnensis L. $\odot$

Serratula coronata L. y)

- heterophylla Desf. 24

- tinctoria L. 2f

Silphium laciniatum L. 24

- integrifolium Mchx. 2)

- perfoliatum L. 2)

- terebinthinaceum L. ข|

- ternatum Retz. \%) 
Silphium trifoliatum L. ข|

Solidago canadensis L. ?)

Sonchus palustris L. 이

Sphenogyne speciosa Maud. $\odot$

spilanthes oleracea L. $\odot$

Spitzelia cupuligera Dur.

Tagetes erecta L. $\odot$

- patula L. $\odot$

- signata Bart1. $\odot$

Telekia cordifolia Dec. 의

- speciosa Baumg. 이

Tragopogon porrifolius L. $\odot$

Tripteris cheirantifolia Schultz $\odot$

Urospermum picroides Desf.

Xanthium macrocarpum Dec. $\odot$

- spinosum L. $\odot$

Xeranthemum cylindraceum Smth. $\odot$

Ximenesia encelioides Cav. $\odot$

Zacintha verrucosa Gart.

Zinnia pauciflora L. $\odot$

- verticillata Andr. $\odot$

\section{Convolvulaceae.}

Convolvulus tricolor L. $\odot$

Ipomoea atropurpurea Chois. $\odot$

- purpurea Lam. $\odot$

- tricolor Cav. $\odot$

Quamoclit coccinea Moench $\odot$

\section{Cornaceae.}

Benthamia fragifera Lindl. $\hbar$

\section{Crassulaceae.}

Sedum album L. 9f

- coeruleum Vahl 24

- palustre L. $\odot$

Tetragonia crystallina Herit. $\odot$

- expansa Ait. $\odot$

\section{Cruciferae.}

Alyssum calycinum L. $\odot$

Arabis albida Stev. 2)

- alpina L. ?)

- bellidifolia Jacq. 9f

- blepharophylla Hook. 과

- ciliata R. Br. 24

- muralis Bertol. क)

- pendula L. ?

- rosea Dec. ?f

- sagittata Dec. ข|

Aubrietia Columna Guss, if

- deltoidea Dec. 2)

- - var Morreana Dec. 2|
Aubrietia graeca Griesb, ?|

- grandiflora hort. 와

Barbarea arcuata Rchbch. -(

- vulgaris R. Br. 24

Berteroa incana Dec. ㅇ

Biscutella apula L. ()

- auriculata L. $\odot$

- eriocarpa Dec. $\odot$

Brassica alba Boiss. $\odot$

- oleracea L. $\odot$

- - var acephala L. $\odot$

- _ - bullata L. $\odot$

- - plumosa L $\odot$

- - - prolifera L. $\odot$

- nigra Koch $\odot$

- Sinapistrum Boiss.

Bunias Erucago L. $\odot$

- orientalis L. $\odot$

Cakile maritima Scop. $\odot$

Camelina microcarpa Andrz.

- sativa Crantz $\odot$

Capsella bursa pastoris Moench. $\odot$

Cardamine chenopodifolia Juss. 이

Cheiranthus Cheiri L. $\odot$

- mutabilis 1'Hérit. $\odot$

- Senoneri Sart. $\odot$

Crambe cordifolia Stev 의

- grandiflora Dec. 24

- hispanica L. $\odot$

Draba Aizoides L. 이.

- subamplexicaulis C. et Mey. ㄱ

Erysimum australe Greg. $\odot$

- cheiranthoides Crantz. $\odot$

- helveticum Dec. $\odot$

- orientale Fisch. et Mey. $\odot$

- ochroleucum Dec. $\odot$

- perfoliatum Crantz. $\odot$

- Perofskianum Fisch. et Mey. $\odot$

- pulchellum Willd. $\odot$

- strictum Gaert.

Hesperis fragrans Fisch. 9

- matronalis L. 2f

Hymenophysa pubescens Meyer -)

Iberis amara L. $\odot$

- Garrexiana All. $\hbar$

- pinnata L. $\odot$

- saxatilis L. $\hbar$

- sempervirens L. $\hbar$

- umbellata L. $\odot$

Isatis dasycarpa Ledeb. $\odot$

- tinctoria L. $\odot$

Koniga libyca Vahl.

Lepidium campestre R. Br. $\odot$

- crassifolium R. K. 2)

- Draba L. $\odot$

- graminifolium L. 97

- latifolium L. 2f

- ruderale L. $\odot$

- sativum L. $\odot$

Lunaria biennis Moench. $\odot$ 
Matthiola sinuata R. Br. $\odot$

Moricandia hesperidiflora Dec. $\odot$

Myagrum perfoliatum L $\odot$

Nasturtium amphibium $\mathrm{R}$. $\mathrm{Br}$. 2)

Notoceras canariensis R. Br. $\odot$

Ochthodium aegypticum Dec. $\odot$

Rhaphanus caudatus L. $\odot$

Sisymbrium Irio L. $\odot$

- tanacetifolium L. 의

Zillia myagroides Forsk.

\section{Cucurbitaceae.}

Bryonia dioica Jacq. ?

Cucurbita maxima Duch. $\odot$

- Pepo L. $\odot$

- - americana.

- turbaniformis.

Cyclanthera explodens Naud. $\odot$

Kedrostis africana Schrad. 2)

Lagciaria clavata hort.

Luffa cylindrica L.

Momordica Charantia L. $\odot$

- Elaterium L. $\odot$

Sicyos angulatus L. $\odot$

Thladiantha dubia Rgl. 24

\section{Datiscaceae.}

Datisca cannabina L. ข)

\section{Dipsacaceae.}

Cephalaria ambrosioides R. S. 2)

- centauroides R. S. 2)

- Joppensis Coult. $\odot$

- tatarica R. S. ?

Dipsacus fullonum Mill. -(

Morina longifolia Wallich. -

Pterocephalus palaestinus Coult. $\odot$

Scabiosa atropurpurea L. (-

- caucasica M. B. 24

- prolifera L. $\odot$

\section{Droseraceae.}

Drosera capensis L. ข|

- binata Labil. of

- spathulata Labill..94

\section{Ericaceae.}

Gaultheria Shallon Pursh. h Rhododendron arboreum Sm. $\hbar$

- hirsutum L. $\hbar$

- ferrugineum L. $\hbar$

\section{Euphorbiaceae.}

Euphorbia cyathophora Jacq. (-

- Esula L. 24

- lathyrus L. 9)

- orientalis L. O)

- Myrsinitis L. ข|

- nicaeensis All. 2f

- platyphylla L. of

- trigonocarpa Lood. 9|

- variegata Sims. $\odot$

Phyllanthus juglandifolius Willd. i Ricinus communis L. $\odot$

\section{Gentianaceae.}

Gentiana asclepiadea L. ?

- - albiflora L. 2)

- cruciata L. 2)

- lutea L. 2f

- tibetica King. 29

- Pneumonanthe L. .)

Menyanthes trifoliata L. of

\section{Geraniaceae.}

Erodium gruinum Willd. $\odot$

Geranium aconitifolium L. ')

- canescens Herit. 2y

- cristatum Steven. 94

- erianthum Dec. 2)

- ibericum Cav. 2y

- lucidum L. $\odot$

- pratense fl. albo L. 2)

- - fl. pleno L. 2)

- sanguineum L. 2)

- sylvaticum L. 2)

- Wlassonianum Fisch. 9)

Pelargonium iatrophifolium Dec. $\hbar$

- tomentosum Jacq. $\hbar$

\section{Gesneraceae.}

Martynia fragrans Lindl. $\odot$

- longiflora L. $\odot$

- lutea Lindl. $\odot$

Ramondia pyrenaica Rich. - $)$

Streptocarpus polyanthus Hook.

- Rexii Lindl. -)

- Wendlandii Sprg. 29

\section{Globulariaceae.}

Globularia vulgaris L. 와

\section{Guttiferae.}

Hypericum Androsaemum L. ')

- floribundum Ait. 9)

- grandifolium Salisb. 2)

- hircinum L. 24 
Hypericum olympicum L. 9 perfoliatum L. 9)

- pulchellum L. $)$

\section{Halorrhagidaceae.}

Cercodia erecta Murr. 2)

Hippuris vulgaris L. ?)

\section{Labiatae.}

Betonica hirsuta L. ?)

- officinalis L. 2)

- stricta Ait.

Dracocephalum Ruyschianum L. 2)

- Moldavicum L. $\odot$

Elsholtzia cristata Willd. $\odot$

Hyssopus officinalis L. 2)

Lallemantia iberica Fisch.

- peltata Fisch.

Lamium garganicum L. ?

- Orvala L. 2)

Lavandula vera Dec. थf

- var. media Dec. 2)

- Stoechas L. h

Leonurus Cardiaca L. ?

- capitatus L. 이

- sibiricus L. $\odot$

Lophanthus anisatus Benth. $\hbar$

Lycopus europaeus L. 2f

- exaltatus L. ข|

Marrubium vulgare L. 2)

Melissa officinalis L. 24

Mentha Pulegium L. ข4

Monarda fistulosa L. 24

- mollis L. 24

Nepeta camphorata Boiss. et Heldr. 24

- lanceolata Dec. 2)

- latifolia Dec. 24

- spicata Bernh. y.

- tuberosa L. 24

Origanum vulgare L. 와

Phlomis tuberosa L. श)

Prunella grandiflora L. 의

- vulgaris L. 2)

Salvia Aethiopis L. ㅇ

- Amasiana Ortg. ㄱ)

- argentea L. 24

- candidissima Vahl. - )

- Hormium L. $\odot$

- dumetorum Andr. 2)

- nilotica Murr. ㅇ

- officinalis L. - ㄱ

- pratensis L. ?

- Sclarea L. $\odot$

- verticillata L. 94

- viridis L. $\odot$

Satureja hortensis L. $\odot$

Scutellaria albida L. o)

- alpina L. .)
Scutellaria Lupulina L. 2|

Stachys ambigua Smitt. ?)

- circinnata Herit. 24

- coerulea Burch. 24

- germanica L. 가

- lanata Jacq. ข|

- longifolia Benth. ๑)

- recta L. 2f

- setifera C. A. M. 2)

- sylvatica L. .)

- tuberosa L. .)

Teucrium Botrys L. $\odot$

- Chamaedrys L. ?)

- Scorodonia L. 2f

Thymus vulgaris L. of

\section{Linaceae.}

Linum alpinum L. ๆ

- aquilinum Dec. $\odot$

- cribrosum L. $\odot$

- grandiflorum Desf. $\odot$

- usitatissimum L. $\odot$

\section{Loasaceae.}

Cajophora lateritia Presl. $\odot$

- triphylla var. vulcanica Urb. $\odot$

- urens Urb. $\odot$

Mentzelia Lindleyi Torr. et Gray. 와

\section{Lobeliaceae.}

Lobelia Erinus L. $\odot$

- syphilitica L. 2ㅇ

\section{Malvaceae.}

Abutilon tiliifolium Fisch. $\odot$

Althaea officinalis L. 2)

- rosea Cav. 文

- - fl. nigro Cav. 9

- Taurinensis Dec. of

Gossypium arboreum L. $\hbar$

Hibiscus Trionum L. $\odot$

Kitaibelia vitifolia Willd. 2

Lavatera trimestris L. $\odot$

Malachra alceifolia Jacq. $\odot$

Malope trifida Cav. $\odot$

Malva caroliniana crispa L. $\odot$

- mauritiana L. $\odot$

- nicaeensis L. $\odot$

Pellaea alternifolia Back.

\section{Mimosaceae.}

Acacia giraffae Willd. 의

- horrida Willd. $\hbar$

Mimosa pudica L. $\odot$ 


\section{Myrtaceae.}

Baeckea virgata Andr. $\hbar$

Tristania laurina R. Br. h

\section{Nyctaginaceae.}

Allionia nyctaginea Michx. ?)

Mirabilis dichotoma L. $\odot$

- Jalapa L. $\odot$

- longiflora L. $\odot$

Oxybaphus angustifolius Pursh. 2)

- Cervantesii Lagas. 2f

- cucullatus Choisy.

\section{Nymphaeaceae.}

Nymphaea coerulea Savign. -9)

\section{Onagraceae.}

Clarkia elegans Dougl. $\odot$ - pulchella Pursh.

Cuphaea procumbens Cav. $\odot$

- Zimapani E. Morr.

Epilobium Dodonaei Vill. of

- trigonum Schrank. 이

Fuchsia microphylla H. B. †

- procumbens R. Cunn. h

Gaura parviflora Hook. $\odot$

Godetia amoena var. Lindleyana Spach. $\odot$

- purpurea Sweet. $\odot$

Jussiaea repens L. ?|

Lopezia racemosa Cav. $\odot$

Lythrum Salicaria L. . tomentosum Mill. of

Oenothera biennis L. $\hbar$

- elata H. B. 2)

- fruticosa L. \%4

- Fraseri Pursh. 이

- glauca Mchx. 2)

- missouriensis Sm. $\odot$

- mollissima L. $\odot$

- muricata Murr. $\odot$

- odorata Jacq. थf

- pusilla Mchx. ข|

- purpurea Curt. $\odot$

- speciosa Nutt. 2f

\section{Orobanchaceae.}

Orobanche arenaria Borgh. 9)

- ramosa L. .

\section{Oxalidaceae.}

Biophytum proliferum D. C. 9f - sensitivum D. C. 24

Oxalis stricta L. .)
Papaveraceae.

Argemone mexicana L. ()

- sulphurea Sweet. $\odot$

Bocconia microcarpa Mey. ?|

Chelidonium majus L. of

- - var. laciniata L. of

Eschscholtzia californica Chmss. (-

Glatucium corniculatum var. tricolor.

Curt. $\odot$

- fulvum Smith.

- luteum Scop.

Papaver alpinum L. 2)

- Apulum Tenor. $\odot$

- Argemone L. $\odot$

- bracteatum Lindl. 2)

- cambricum L. $\odot$

- dubium L. 24

- Heldreichii Boiss, - y

- hybridum L. $\odot$

- nudicaule L. 9)

- orientale L. शf

- pilosum Sib. et Sm. 29

- Rhoeas L. $\odot$

- somniferum L. $\odot$

\section{Papilionaceae.}

Anisolotus Wrangeliana Bernh. $\odot$

A throlobium scorpioides Dec. $\odot$

Astragalus baeticus L. $\odot$

- Cicer L. 24

- galegiformis L. \%

- ponticus Pall. 가

- stipulatus Don. थ|

Baptisia australis R. Br. 9)

- minor Lehm. 24

Callistachys lanceolata Dec. $\hbar$

- retusa Lodd. $\hbar$

Caragana Altagana Poir. $\hbar$

Cicer arietinum L. $\odot$

Clitoria ternata L. 2)

Desmodium canadense Dec. ㄱ)

Dolichos Catjang L. $\odot$

Dorycnium suffruticosum Vill. $\hbar$

Galega officinalis L. 2)

Genista canariensis L. $\hbar$

- elatior Koch. 9)

- radiata Scop. 2)

- scoparia L. 2y

- tinctoria var. virgata L. W. h

Glycyrrhiza echinata L. ?|

Hedysarum grandiflorum Pall. 의

- obscurum L. ?|

Indigofera Doiaca Hamilt. $\mathrm{h}$

- pulchella Roxb. $\hbar$

Lathyrus Aphaca L. $\odot$

- articulatus L. $\odot$

- Clymenum L. $\odot$

- latifolius L. 2)

- mexicanus Wender. $\odot$ 
Lathyrus Nissolia L. $\odot$

- Ochrus Dec. $\odot$

- pseudaphaca L. $\odot$

- tuberosa L. 9f

Lotus ornithopodioides L. $\odot$

Lupinus albus L. $\odot$

- Cruikshanksii Hook. $\odot$

- perennis L. 이

- polyphyllus Dougl. ㅇ

- succulentus Dougl. 2)

- Hartwegii Rgl. $\odot$

Medicago echinata L. $\odot$

- falcata L. 2)

- glutinosa Bbrst. 2)

- intertexta Willd.

- marginata Willd. $\odot$

- scutellata Lam. $\odot$

Melilotus coerulea Lam. $\odot$

- macrorrhiza Pers. 2)

- officinalis Pers. 2)

Onobrychis Caput-galli Lam. ㅇ

- Crista-galli Lam. $\odot$

Ononis fruticosa L. 2)

- spinosa fl. albo L. 이

Orobus laevigatus W. K. थ

- flaccidus Kit. 24

- luteus L. 2)

- niger L. 2.

- vernus L. 2)

Phaseolus capensis Thbg. $\odot$

- compressus Dec.

- multiflorus Lam. $\odot$

- vulgaris var. Zebrina L. $\odot$

Pisum sativum L. $\odot$

Pocockia cretica Dec. $\odot$

Scorpiurus muricata L. $\odot$

- subvillosa L. ?

Securigera Coronilla Dec. $\odot$

Sophora alopecuroides L. ㅇ

Spartianthus junceus Link. $\hbar$

Sutherlandia frutescens R. Br. $\hbar$

Swainsona coronillifolia Salisb. it

Tetragonolobus biflorus Sering. $\odot$

- purpureus Moench. $\odot$

Trifolium alexandrinum L. $\odot$

- alpestre L. 29

- fragiferum L. ๆ)

- pannonicum L. 21

- procumbens L. $\odot$

- repens L. var. macrorrhiza Boiss 의

- rubens L. 2

Trigonella corniculata L. $\odot$

- Foenum graecum L. $\odot$

Vicia cassubica L. ?)

- Cracca L. 2)

- Faba L. $\odot$

- ferruginea Besser $\odot$

- Orobus Dec. ?

- pisiformis L. थ
Vigna glabra Sav. $\odot$

\section{Passifloraceae.}

Passiflora gracilis Link. $\odot$

- macrocarpa Mast.

\section{Phytolaccaceae.}

Phytolacca Kaempferi A Gray. 2)

Rivina aurantiaca Warsz.

- brasiliensis Nocca. $\hbar$

- laevis L. $\hbar$

- humilis L. $\hbar$

\section{Piperaceae.}

Peperomia maculosa Hook. 이

\section{Plantaginaceae.}

Plantago alpina L. ข|

- amplexicaulis Cav. ㅇ

- Lagopus L. 94

- lanceolata L. 2f

- maritima L. 2)

- minima L. ㅇ

- Psyllium L. 2y

- subulata L. 24

\section{Plumbaginaceae.}

Statice elata Fisch. 2f

- latifolia Sm. ำ

- Limonium L. ข|

- sinuata L. $\odot$

\section{Polemoniaceae.}

Phlox decussata Hort. 2)

- Drummondï Hook. $\odot$

- repens Michx. 24

- suaveolens Ait. 9f

\section{Polygonaceae.}

Polygonum cymosum Desf. -)

- divaricatum L. 24

- orientale L. $\odot$

- viviparum L. ?

Rheum officinale Baill. 의

- undulatum L. 9

- Rhaponticum L. 2/

Rumex alpinus L. शै

- conglomeratus Roth 2

- crispus L. 24

- salicifolius Weinm. 2 


\section{Portulacaceae.}

Calandrinia sericea Hook. ?) Portulaca grandiflora Camb. - oleracea L. $\odot$

\section{Primulaceae.}

Androsace lactiflora Fisch. 2) Dodecatheon Jeffreyi Moore ? Lysimachia quadrifolia L. 2/ - thyrsiflora L. .)

- verticillata Bbrst. 2f

Primula Auricula L. ㅇ

- japonica A Gray. ?4

- officinalis Jacq. ๆ

- rosea L. 21

Samolus Valerandi L. 의

\section{Pyrolaceae.}

Pyrola rotundifolia L. ?f

\section{Ranunculaceae.}

Aconitum Anthora L. 2)

- barbatum L. 2)

- Lycoctonum L. 와

- Napellus L. 2y

- paniculatum Lam. 9)

- septentrionale Mart. 이

- Stoerkianum Rchb. 9)

Actaea erythrocarpa Fisch. 2)

- spicata L. 9|

Anemone alpina L. थ)

- fulgens Gay. ?|

- multifida Poir. 2f

- palmata L. 2)

- sylvestris L. 2y.

Aquilegia alba L. \%)

- alpina L. 2f

- atrata C. Koch 2)

- Bertolonii Schott. 24

- blanda v. Houtt. थ

- Burgeriana S et Z. 2|

- californica Led. 24

- coerulea James, 2y

- chrysantha A. Gray of

- fragrans Benth 2)

- flavescens Watts.

- flabellata S. et Z. 2f

- formosa Fisch. 2f

- glandulosa Fisch. 2f

- lutea Lam.

- Skinneri Hook. ?f

- sibirica Lam. \%

- vulgaris L. 9)

- - fl. pleno L थ
Caltha palustris L. ?|

Cimicifuga foedita L. ๆ)

Clematis alpina Mill. of

- angustifolia Jacq. थf

- aethusifolia Turcz. 9f

- gebleriana Bong. .)

- flammula L. 2)

- heracleifolia D. C. .)

- mongolica Rgl. ㄱ.

- orientalis L. 2y

- recta L. \%

- - var. Flammula L. 9)

Delphinium Ajacis L. $\odot$

- cheilanthum Fisch. 2)

- elatum L. 의

- - var. montanum L. .)

- - var. leucopetalum Rehb. 2y

- - speciosum Bbrst. 2)

- formosum L. 2)

- grandiflorum L. ?)

- palmatifidum Dec. 29

- Woehleri Lam. 2)

Nigella damascena L. $\odot$

- orientalis L. $\odot$

- sativa sem. nigro L. $\odot$

Paeonia albiflora Pall. 2)

- arietina Anders.

- commutata Wender. ?

- corallina Retz. 24

- decora Anders. 24

- humilis Retz. 9y

- paradoxa Anders. 94

- peregrina Mill. ขy

- pubens Sims. 기

- puberula Torn. 29

- tenuifolia L. 9|

- triternata Pall. 9)

Ranunculus arvensis L. $\odot$

- caucasicus M. B. 2!

- muricatus L. $\odot$

- sardous Crantz. 2|

- Stevenii Andrz. of

Thalictrum aquilegifolium L. 2)

- cinereum Desf. 2)

- cornutum L. 2)

- dubium Bung. 9

- elatum Jacq. 2f

- foetidum L 9

- galioides Nestl. 24

- glaucum Desf. ㅇ

- heterophyllum Lejeun. 2f

- intermedium L. 2)

- minus L. 24

- purpurascens L. 94

- simplex L. 아

- sylvaticum Hook. श)

Trautvetteria palmata Fisch. et Meyer.9

Trollius altaicus May. of

- caucasicus Stev. 9f

- europaeus L. 2f 
Resedaceae.

Reseda alba L. $\odot$

- abyssinica Fresen. $\odot$

- odorata L. $\odot$

- luteola L. $\odot$

\section{Rhamnaceae.}

Pomaderris elliptica Labill. $\hbar$

\section{Rosaceae.}

Acaena Sanguisorba Vahl. भ|

Agrimonia dahurica Willd. of

- eupatoria L. 29

- pilosa Ledeb. 2)

- repens L. 24

Alchemilla alpina L. 9

- fissa Günth. 2)

- vulgaris L. 2f

Dryas octopetala L. 9

Geum carolinianum Walt. 9

- coccineum Sm. 2)

- japonicum Thbg. ?

- ranunculoides Sering. 94

- rivale L. 9|

- strictum Ait. 24

Potentilla alpestris Hall. 94

- argentea L. 2)

- atrosanguinea Don. 9y

- collina Wibel. 9

- delphiniensis Gr. Good 2

- formosa L. 24

- fruticosa L. 94

- grandiflora L. b. fragiformis Willd. 9

- haematochrus Lehm. भf

- Hippiana Lehm. 29

- Hookeriana Lehm. 2

- inclinata Vill. 94

- megalontodon Lehm. 9)

- norvegica L. $\odot$

- procumbens Sibth. ㄱ

- recta L. 24

- rupestris L. var. grandiflora L. 94

- Wrangeliana F. et M. 2)

Poterium muricatum Spach of

Rosa laxa L. $\hbar$

- rugosa Retz. $\hbar$

Sanguisorba canadensis L. ')

- officinalis L. 24

- mauritiana Desf. ')

- tenuifolia Fisch. 91

Spiraea grandis Sweet of

- filipendula fl. pl. L. 24

\section{Rubiaceae.}

Asperula arvensis L. 2)

- ciliata Rochel. of
Asperula tinctoria L. 9)

- tyraica Bess. 9)

Crucianella aegyptiaca L. $\odot$

- gilanica Trin.

Galium boreale L. ?)

Nertera depressa Banks. 94

Phyllis Nobla L. †

Schenckia Blumenaviana L. $\odot$

Sherardia arvensis L. $\odot$

\section{Rutaceae.}

Dictamnus albus L. 2)

- fraxinella Pers. 94

Diosma alba Thbg. $\hbar$

Ruta graveolens L. थ|

Skimmia japonica Thbg. $\hbar$

Xanthoxylon americanum Mill. $\hbar$

\section{Sapindaceae.}

Koelreuteria paniculata Lam. ti Xanthoceras sorbifolia Bunge $\hbar$

\section{Saxifragaceae.}

Bergenia orbicularis Moench. $\hbar$ Francoa appendiculata Cav. $\hbar \iota$ Heuchera alba Gray. 9 - sanguinea Gray. 94 Mitella pentandra Hook. 94 Parnassia palustris L. 2) Saxifraga Aizon L. ขf

- Cotyledon L. 가

_ - var. major L. 9

- - minor L. 9f

- cartilaginea Willd. 4

- ceratophylla Ait. 29

- cymbalaria L. 24

- hirsuta L. 2|

- longifolia Lapeyr. 9

- umbrosa L. 24

- - fol. varieg. L. 9

Tiarella cordifolia L. 94

\section{Scrofulariaceae.}

Antirrhinum calycinum Vent. $\odot$

\section{- majus L. 2)}

Calceolaria scabiosifolia Sms. $\odot$

Chelone barbata Cav. 2f

Collinsia bicolor Benth. $\odot$

Digitalis ambigua L. 94

- grandiflora Lam. 2

- lutea L. 24

- parviflora Jacq. 4

- purpurea L. $\odot$ 
Digitalis purpurea var. alba L. $\odot$

- - var. gloxiniifolia Hort. $\odot$

Hebenstreitia alba Jacq. 2)

Linaria amethystea Hoffmgg.

- aparinoides F. G. Dietr. $\odot$

- triphylla Willd. $\odot$

Melampyrum pratense $\mathrm{L}$. $\odot$

Mimulus cupressus L. $\odot$

- luteus L. 9)

- tigrinus L. $\odot$

Penstemon barbatus Roth. 2)

- digitalis Nutt. 24

- glandulosus Grah. 9

- glaucus Lindl. 24

- pubescens Soland. 24

- secundiflorus Benth. 2f

- speciosus Doug1. ㄱ

Scrophularia nodosa L. ㅇ

- vernalis L. 24

Torenia asiatica L. $\odot$

- edentula Benth. $\odot$

- exappendiculata Rgl. $\odot$

- Fournieri Lindl. $\odot$

Verbascum Blattaria L. $\hbar$

- Kanitzianum Link. $\odot$

- nigrum L. $\odot$

- phlomoides L. $\odot$

- phoeniceum L. $\odot$

- Thapsus L. $\odot$

- thapsiforme Schrad. $\odot$

- - var. grandiflorum Schrad. (-

Veronica Anagallis L. ?)

- aquatica Bernh. ?|

- Bachofiana Hauff. 2f

- Beccabunga L. 24

- corymbosa Hort. $9 y$

- crenulata Hoffm. 9f

- dichrus Schott et Kotschy.

- gentianoides Vahl of

- grandis Ehrh. 24

- incana L. 24

- longifolia fl. albo L. 24

- mollis W. थै

- officinalis L. ?

- pectinata L. 와

- polystachya Hort. थ4

- prostrata L. 24

- serpyllifolia L. 94

- sibirica L. 94

- spicata L. ㅇ

- spuria L. 94

- Teucrium L. 9f

- Tournefortii Gmel. 9)

- urticifolia L. 24

- virginica L. 94

\section{Solanaceae.}

Atropa Belladonna L. 9!

Capsicum annuum L. $\odot$
Capsicum annuum longum rubrum $\odot$

- - myrtilliforme $\odot$

- nigrum Willd. $\odot$

Datura Stramonium L.'- )

- Tatula L. $\odot$

Hyoscyamus albus L. $\odot$

- orientalis Bbrst. 9|

- niger L. $\odot$

Lycopersicum cerasiforme var. rubra Don $\odot$

- pyriforme var. lutea

- - - rubra

Nicandra physaloides Gaert. $\odot$

Nicotiana acuminata var. silenifolia. L. $\odot$

- alata Link et Otto. 94

- colossea L. $\odot$

- Langsdorffii Nees. $\odot$

- macrophylla Spr.

- rustica L. $\odot$

- Sanderiana S. $\odot$

- Tabacum L. $\odot$

Petunia violacea Hook. $\odot$

Physalis Franchetti Mast. $\odot$

- peruviana L. $\odot$

Solanum Dulcamara L. $\odot$

- Melongena L. $\odot$

- miniatum Bernh. $\odot$

- macrocarpum L. h

- marginatum L. $\hbar$

- nigrum L. $\odot$

- - var. pumila L. $\odot$

- Pseudocapsicum L. $\hbar$

\section{Thymelaeaceae.}

Daphne Laureola L. $\hbar$

- Mezereum L. $\hbar$

\section{Tiliaceae.}

Aristotelia Macqui L'Herit. $\hbar$

\section{Tropaeolaceae.}

Tropaeolum majus L. $\odot$

\section{Umbelliferae.}

Anethum graveolens L. $\odot$

Astrantia Biebersteinii Fisch. et Mey. 94

- carniolica Jacq. 94

- helleborifolia Salisb. 94

- major L. ?4

- minor L. 94

Athamanta Matthioli Wulff, 9f

Bupleurum falcatum L. 2f 
Bupleurum longifolium L. ?)

- multinerve Dec. 2)

- rotundifolium L. $\odot$

- ranunculoides L. $\odot$

- sachalinense F. Schmidt. 2)

Carum Bulbocastanum Koch. 2)

- Carvi L. $\odot$

Chaerophyllum aureum L. 9)

- bulbosum L. 이

Cicuta virosa L. 2)

Conium maculatum L. ค)

Coriandrum sativum L. $\odot$

Cryptotaenia canadensis Dec. थ|

Eryngium agavifolium L. .)

- amethystinum L. 이

- coeruleum Bbrst. थf

- dichotomum Desf. 2

- giganteum M. B. 94

- maritimum L. 2)

- pandanifolium Chmss. 94

- planum L. 2)

- Oliverianum Delar. 2)

- Sanguisorba Chmss. 2

- Serra Chmss. et Schl. 21

- tripartitum Desf.

Ferula Ferulago L. 9)

- tingitana L. 9)

Foeniculum capillaceum Gilib. 와

- vulgare Gaert. $\odot$

Heracleum barbatum Ledeb. 9)

- Mantegazzinum Levier. 94

- pubescens M. B. 24

- sibiricum L. 9)

- sphondylium L. ?)

- villosum Fisch et Schult. 9

Imperatoria Ostruthium L. 2)

Laserpitium intermedium L. .

Levisticum officinale Koch. of

- paludapifolium Lam. ㅇ

Libanotis vulgaris Dec. 2)

Ligusticum Seguieri Koch. 2f

Lophosciadium meifolium Vent.

Malabaila Hacquetii Tausch. (-)

Meum athamanticum Jacq. $\odot$

Myrrhis odorata Scop. (-)

Oenanthe aquatica Lam.

- Lachenalii Gmel.

- fistulosa L. 2)

- pimpinelloides L. 9

- peucedanifolia Pollich. 9|

Pachypleurum alpinum Ledeb. 9)

Pastinaca sativa L. $\odot$

Petroselinum sativum Hoff. $\odot$

Pimpinella Anisum L. $\odot$

- magna L. 24

- rotundifolia Bbrst. 2)

- Saxifraga L. 아
Prangos thapsoides Dec. 2)

Rumia leiogona C. A. Mayer. 2)

Selinum Carvifolia L. ?|

Seseli glaucum Dec, 2)

- gummiferum Sm. 9y

- Libanotis C. Koch. 2)

- montanum Dec. ?|

- tenuifolium Ledeb. 9|

Siler aquilegifolium Gaert. 94

Sium latifolium L. 2)

- Sisarum L. 9)

Trinia Kitaibelii Bbrst.

Xanthogalum purpurascens W.

Zizia aurea Koch.

- integerrima Dec. 2)

\section{Urticaceae.}

Urtica balearica L. $\odot$
- cannabina L. 9
- pilulifera L. $\odot$

\section{Valerianaceae.}

Fedia graciliflora Fisch. et Mey. $\odot$

Centranthus ruber Dec. 2)

- - var. alba Hort. 9!

- - - rosea Hort. 9)

Valeriana officinalis L. 9f

- sambucifolia Mill. of

Valerianella dendata Dec. $\odot$

\section{Verbenaceae.}

Shuttleworthia pulchella Meissn. (-

Verbena officinalis L. 2)

- venosa Hook. 94

\section{Violaceae.}

Viola calcarata L. श)

- collina Bess. 24

- cornuta L. 2)

- dactyloides R. et Sch. 2).

- elatior Fries. 91

- macedonica Heldr. 24

- mirabilis L. 2)

- odorata L. 29.

- pinnata L. 9)

- prionantha Bunge ?

- sylvestris Lam. 24

\section{Zygophyllaceae.}

Tribulus terrestis L. $\odot$ 


\section{Anhang.}

Ableger können abgegeben werden von folgenden Sedum- und

\section{Sempervivum-Arten :}

Sedum acre L. 의

- Aizon L. 9)

- album L. 9

- boloniense Loisel. 아

- calabricum Ten. 24

- cruciatum Desf. 29

- cyaneum Rad. भ

- elegans Lejeun. 24

- Ewersii Lood. 24

- Kamtschaticum Regel. .)

- Maximowiczii Regel. -4

- purpurascens Link. 9f

- reflexum Willd. 'f

- Rhodiola Dec. 94

- roseum Stev. 9)

- sexangulare L. 94

- speciosum hort. 94

- spurium Bbrst. 94

- Telephium L. 94

- Wallichianum Hook. 94

Sempervivum albidum Lehm. et Schnittsp. 24

- acuminatum Schott. 2|

- arachnoideum L. भ|

- arenarium Koch. 24

- assimile Schott. 9|

- blandum Schott. 9|

- Boutignyanum Lk. et Sch. 9f

- Braunii Funk. 24

- californicum hort. 94

- Comollii Rota. 2y

- debile Schott. 9f

- Delastrii Lehm. et Schnittsp. 9f

- dolomiticum Hausm. 24

- fimbriatum Lehm. et Schnittsp. 29
Sempervivum flagelliforme Fisch. 94

- Funkii A. Br. 9/

- - brachypetalum A. Br. 94

- glaucum Ten. ?

- globiferum L. 9

- grandiflorum Haw. 9

- heterotrichum Schott. 94

- Heuffelii Schott. 94

- hirtum L. 29

- hispidulum Schott. 9

- Laggeri Schott. ?

- Mettenianum Lehm. -)

- modestum Jord. 97

- montanum L. 94

- patens Grieseb. 9f

- piliferum hort. 24

- Reginae hort. 2f

- Requienii R. S. 9y

- robustum Himp. 9

- rubicundum Schur. 9|

- ruthenicum hort. 94

- Schlehani Schott. 24

- Schottii Br. 2I

- Schnittspahnii Dill. of

- soboliferum Sims. 91

- spinulifolium hort. 9y

- stenopetalum Lehm. et Schnittsp. 2)

- tectorum L. ?

- tomentosum Lehm. 94

- triste hort. 2/

- Verloti Jord. 94

- - Borserii Jord. 9

- - glaucum Jord. 9

- violaceum Blasius. 94

- Wulfenii Hopp. 94

Bestellungen werden bis zum 15. Februar erbeten.

\section{Prof. Dr. M. Möbius}

Director.
Rudolf Günther

Obergärtner. 
Acacia armata $\boldsymbol{R} \cdot \boldsymbol{B r}$.

- lophantha $W$.

- - var. coarctata. stricta $A n d r$. nndulata $W$

Acaena cylindrostachys $R$. et $P$. Achillea Ageratum $\boldsymbol{L}$.

- odorata $L$

Aehyranthes axillaris $W$.

- brachiata $L$.

Aconitum Anthera $L$.

- neubergense $D_{e c}$.

Acroglochin chenopodioides Schrd.

Actaea racemosa $L$. spicata $L$

Adonis autumnalis $\boldsymbol{L}$.

Aegilops eylindrica Host.

- ovata $I$.

- squarrosa $L$.

- triencialis $L$

Agrimonia oderata Camer.

Agrostis coromandelinia Retz. versicolor Stev.

Allionia nyctaginea Melhx.

Allium acutangulum $W$.

- baicalense $W$.

- cernuum Roth.

- fistulusum $\boldsymbol{L}$.

- fragrans Fent.

- nenpolitanum Cyrill.

- Ophisscorodon Link.

- pusillum Cyrill.

- striatum Jacq.

- tenuifiorum Ten.

- tenuissimum $W$.

- Vietorialis $L$.

Alstroemeria Pelegrina $L$.

Althaea cannabina $L$.

- Froloviana Fisck.

- Sieberi $L k$.

Alyssum argenteum $W$.

- edentulum Kit.

- murale.

- rostratum Stev.

- saxatile $\boldsymbol{L}$.

- serpyllifolium Desf.

Amarantus angustifolius $M, B$.

- flavus $\boldsymbol{L}$.

- paniculatus $\boldsymbol{L}$.

- spinosus $L$.

- sylvestris Desf.

- tricolor $L$.

Amethystea coerulea $\boldsymbol{L}$.

Ammannia latifolia $\boldsymbol{L}$.

Ammi majus $L$.

- Visnaga Lam.

Anmobium alatum $\boldsymbol{R}$. $B r$.

Anaeyclus Pyrethrum Lk.

Anehusa asperrima Delil.

- hybrida Ten.

- paniculata Ait.

- rosea $M . B$.

- sempervirens $\boldsymbol{L}$.

Androsace lactiflora Fisch.

- septentrionalis $\boldsymbol{L}$.

Androsaemum officinale $A l l$.

Andryala cheiranthifolia Herit.

Anemone ochotensis Fisch.

Angelica verticillaris $\boldsymbol{L}$.

Anoda hastita Cav.

Anthemis rigescens $W$

- ruthenica $\boldsymbol{M}$. $\boldsymbol{B}$.

- secundiramea Biv.

Anthyllis tetraphylla $L$.
Antirrhinum elegans e $h$. Neap. - sempervirens Lapeyr.

Apargia cancasica $M$. B.

- crispa $W$.

- macrorhiza Günth. tuberosa $W$.

Aquilegia atropurpurea $W$.

- canadensis $\boldsymbol{L}$.

- glandulosa Fisch. speciosa H. Marb.

Arabis alpina $L$

- collina Ten.

- hirsuta Scop.

- pendula $L$.

- Turrita $L$

Arachis hypogaea $L$.

Ardisia crennlata Vent.

Arenaria calycina Poir.

Argemone Barklayana Grah.

- mexicana $L$.

- - $\beta$. albiflora.

Armeria alliacen $W$. alpina $W$.

- denticulata Bertol.

- dianthoides Horn.

- maritima $W$.

- parpurea H. Erl. scorzoneraefolia.

Artemisia Sieversiana Ehrh.

Arum Dracunculas $L$.

Asclepias angnstifolia $W$. curassavica $L$. incarnata $L$.

Asphodelus clavatus Roxb.

- creticus Lam.

- luteus $L$.

- tauricas $M . B$.

Astragalus alopecuroides $L$

- asper Jacq.

- baeticus $L$.

- carolinianus $L$

- Cicer L.

- galegiformis $\boldsymbol{L}$.

- pentaglottis $L$.

- purpureus Lam.

- sulcatus $L$.

- tribuloides.

Athamanta Matthioli $\boldsymbol{W u l f}$. stricta Led.

Atheropogon apludoides $W$.

Aubrietia Columnae Guss. deltoidea Dec.

Avena distichophylla Vill.

- planieulmis Schrad. rigida $M . B$.

Balbisia elongata $W$.

Barbarea praeeox $\boldsymbol{R}, \boldsymbol{B r},{ }^{1}$ )

Barkhansia alpina $L k$.

- graveolens Rchb.

- leontodontoides Rchb. rubra $L k$.

Basella rubra $L$.

Betonica incana Ait.

- orientalis $\boldsymbol{L}$.

Bidens bipinnata $L$

- grandiflora Balb.

- leucantha $\boldsymbol{W}$.

Billardiera scandens $S \mathrm{~m}$.

Billbergia zebrina Lindl.

Biscutella eriocarpa Dec.

Biserrula Pelecinus $\boldsymbol{L}$.

Blumenbachia insignis Schrad.

Becconia frutescens $L$.

Boerhavia hirsuta $\boldsymbol{L}$.
Borreria capitellata Cham.

Brachypodium asperum $\boldsymbol{R}$. et Sch.

- longifolium $P . B$.

Briza maxima $L$.

Bromus alopecnroides Poir.

- angnstifolius $M . B$.

- ciliatus $L$.

- commutatus Schrad.

- gracilis $W$

- lanuginosus Poir.

- laxus Horn.

- loliacens Ten.

- mollissimus Led.

- parviflorus Desf.

- pendulinus Sess. pubescens Muehlenb. rupestris Host. stenophyllns $\mathrm{L} k$.

Bulbine annua $W$.

Bunium peucedanoidés $M . B$.

Buphthalmum salicifolium $\boldsymbol{L}$.

- spinosum $\boldsymbol{L}$.

Bupleurum heterophyllam $L k$. multinerve Dec. protractum $\boldsymbol{L} k$.

Cacalia sonchifolia $\boldsymbol{L}$

Cakile perennis $D_{e c}$.

Calandrinia compressa Schrad.

- discolor Schrad.

glauca Schrad. grandiflora Lindl

Calceolaria pinnata $L$.

Caldasia heterophylla $W$.

Calendula aegyptiaca Desf. pluvialis $L$. stellata Cav.

Callistachys lanceolata Vent.

Camelina dentata Pers.

Camellia japonica $\boldsymbol{L}$.

Campanula aggregata $W$.

- betonicaefolia $M . B$

- divergens $\boldsymbol{W}$.

- hybrida $L$.

- latifolia $L$

- Lorei Pollin.

- macrantha Hornem.

- obliquifolia Ten.

- peregrina $L$.

- sibirica $L$.

- stylesa Lam.

- urticaefolia Schmidt.

Canna Iagunensis Lindl.

- limbata Roso.

- Intea Rose.

- speciosa Rosc.

Capraria biflora $L$.

Capsicum baccatum $\boldsymbol{L}$.

- bicolor Jacg.

- ciliare $\boldsymbol{W}$ pendulum $W$.

Cardiospermum Halieacabum $\boldsymbol{L}$

Carduus Argyroa Biv.

Carex Agastachys $\boldsymbol{L}$.

- divulsa Good.

- juncea $\boldsymbol{W}$.

- laevigata $\boldsymbol{S m}$.

- nutans Host.

Carthamns tinctorins $L$.

Carum Bulbocastanum Koch.

Cassia Tora Ait.

Cassine Maurocenia $L$,

Catananche coerulea $\boldsymbol{L}$

- Intea $L$.

Caucalis grandiflera $\boldsymbol{L}$.

Caucalis platycarpos $L$.

Celosia eastrensis $L$.

- cristata $\boldsymbol{L}$.

- margaritacea $L$. virgata Jacq.

Celsia Areturus $L$. - australis.

- lanata Jacq.

Cenchrus echinatus $\boldsymbol{L}$

Centaurea benedicta $L$.

- calocephala $\boldsymbol{W}$.

- coriacea Kit.

- Crupina $L$.

- eriophora $\boldsymbol{L}$

- ferox Desf.

- melitensis $\boldsymbol{L}$

- moschata $\boldsymbol{L}$.

- rupestris $\boldsymbol{L}$.

- sonchifolia $\boldsymbol{L}$

- Stuebe $\boldsymbol{L}$.

- suIphurea $W$.

- tatarica $L$.

Centrachne viscida Schott.

Centrospermum Chrysanthemum Spreng.

Cerastium davurieum Fisch.

- grandiflorum Kit.

Ceratochloa pendula Schred.

- unioloides Dec.

Cerinthe alpina Kit.

- aspera Roth.

- minor $L$.

Cheirinia crepidifolia $L k$.

Chelidonium laciniatum Mall.

Chelone atropurpurea Don.

- campanulata Cav.

- conferta $\boldsymbol{R} . \boldsymbol{B r}$.

- Digitalis $S p r$.

- hirsuta $L$.

- ovata Dougl.

Chenopodium ficifolium $\mathbf{S} m$.

Chryseis tenuiflora $\boldsymbol{H}$. Jen.

Cicer arietinum $\boldsymbol{L}$.

Cineraria gibbosa Guss.

Cirsium laniflorum $\boldsymbol{M}$. $\boldsymbol{B}$.

- scleranthon $\boldsymbol{M}$. $\boldsymbol{B}$.

- silyboides.

Cistus garganieus Ten.

- vaginatus Ait.

Claytonia perfoliata $D_{0 n}$

Clematis angustifolia Jacq.

- erecta $L$

- Flammula $\boldsymbol{L}$.

- parviflura Dee.

Cleome gigantea $\boldsymbol{L}$.

- graveolens Rafin.

- spinosa $\boldsymbol{L}$.

- violacea $L$

Cneorum tricoccen $L$.

Cnicus spurias Hoffm. syriacus $W$.

Cnidium apioidea $S p r$.

Coix exaltata Jacq.

Collomia grandiflora Dougl.

Colutea arborescens $L$.

- haleppica Lam.

- media $\boldsymbol{W}$.

Comruelina angustifolia $\mathrm{M}$. 
Convolvulus sienlus $L$.

Conyza cardida $\boldsymbol{L}$.

Coreopsis Atkinsoniana Dougl. - Janceolata $\boldsymbol{L}$.

Corispermum canescens Kit.

Coronilla coronata $L$. - glaucir $L$.

- Securidaca $L$.

Corydalis aurea $W$. - sempervirens Pers.

Cosmen bipinnata $W$.

Crambe maritima $L$.

Crepis heterosperma Schrad.

- parviffora Desf.

Critamns davuricus Hoff $m$.

Crucianella angustifolia $L$

Cucubalus barciferus $L$.

Cuenmis prophetarum $L$.

Cucnubita lagenaria $\boldsymbol{L}$.

Cuphea procumbens Cav.

- viscosissima Jacq.

Cyclamen hederaefoliun Ait.

Cynanchum nigrum Pers.

Cynoglossnm apenninum $L$.

- bicolor $W$.

- canescens $W$.

- cheirifolium.

- Dioscoridis Fill.

- sylvaticum $S_{m}$.

Cyperus alternifolius $L$.

- spectnbilis Schreb.

Iatisca cannabian $\boldsymbol{L}$.

Datura ferox $\boldsymbol{L}$.

- Metel $L$.

- Tatula $L$.

Baucns gummifer Lom.

- hispidas Desf.

- muricatus $L$.

Delphinium grandiflorum $\boldsymbol{L}$.

- Requienii Dec.

Desmanthus virgatus $\boldsymbol{w}$.

Wianthus collinus Kit.

- dintinus Kit.

- longicanlis Ten.

- sylvaticus Hopp.

- velutinus Guss.

virginens $L$.

Digitalis ferruginea $\boldsymbol{L}$.

- grandiflora Levm.

- Iaevigata Kit.

- lanata E/arh.

- Intea $\boldsymbol{L}$.

Digitaria aegyptiaca $W$

Diplachne faseicularis $P, B$.

Doliehos brasiliensis.

- Catiang $L$.

- Lablab L.

- melanephthahmos Dec.

Draba aurea $V a h l$.

Dracocephalum canariense $L$.

- canescens $L$.

- Moldaviea $L$.

- nutans $L$.

- peltatum $L$.

Lecremocarpos seaber $R$. et $P$.

thehinops Ritre $L$.

- sphaerocephadus $t$.

Echinospermum Lappala Lefla.

Echitun creticum $\boldsymbol{L}$.

- grandiflorum

- salmanticara Lag

Belipta ereeta $\boldsymbol{L}$.

- Iatifolía $L$.

- prosirata $L$.

Nlemsine ceracana Pers.

- indiea Lam.

- rigida $S p x$.

- Tocusea Ereg.

Riliehrysurn braeteatiun Vent.
Elsholtzia eriatata $\mathbb{W}$.

Elymus arenarius $\boldsymbol{L}$.

- canadensis $L$.

- carolinianus.

- gigantens Vahl.

- Hystrix $\boldsymbol{L}$.

- nardianus H. Genev.

Eruex spinosus Camp.

Epilobium angustissimnu Ait.

Eragrostis abyssinica $L$ l

Eranthis hyemalis Salist.

Erigeron contortus Desf.

- elongatus Led.

- podolicus Bess.

Erodium gruinum Ait.

- mosehatum Ait.

- pulverulentum $W$.

Ervum agrigentinum Guss.

- Ervilia $L$.

Erynginin azureum $H$. Par. giganteum $M$. $B$. planum $\boldsymbol{L}$.

Erysimum altaicum Meyer.

- hieracifolium.

- odoratum Fhrh.

- virgatmun Roth.

Eschscholaia ealifirnica Cham.

Ethulia conyzoides $L$.

Eupatorium ageratoides $L$. paniculatum Mill.

Euphorbia cyathephera Jaeq.

- Humboldtii $\boldsymbol{W}$.

- Lagaseae Spr.

- Lathyris $L$.

- prunifolia Jac\%.

Farsetia clypeata $R$. $B r$.

- eriecarpa Dec.

Fedia Cornucopiae $\mathrm{Fahl}$.

Festuca amethystina Host. - laevigata Gaud. - nutans.

Flaveria repanda $\mathrm{Lag}$.

Forskolea angastifolia Rela.

- tenacissima $L$.

- viridis Ehrenb.

Fragaria indiea $A n d v$.

Fuchsia arborescens Sess.

Fumaria capreolata $L$.

Galardia bicolor Lam.

Galega officinalis $L_{\text {. }}$.

- persica Pers.

- stricta Ait.

Galinsogen trilobata Cav.

Galium infestrum Kit.

- spurium

-verrucosum $s_{m}$.

Garidella Nigellastrnm $L$.

Gaura biennis $\boldsymbol{L}$.

- fruticosi Jacq.

- tripetala Cav.

Gentiana cruciata $L$.

Geranium anemanaefoliam Her.

- carolinianum $L$.

- sanguineum $L$.

- sibiricum $L$.

Geropogon glaber $\boldsymbol{L}$.

Geum coccinenm $S \mathrm{~S}$.

- heterophyllum.

- ranunculoides Ser.

- reptans $L$.

Gilia achilleaefolia Benth.

- inconspicna Hook.

- laciniata $\boldsymbol{R}$. et $\boldsymbol{P}$.

- tricolor Benth.

Gladiolas psittacinus.

Glaucium corniculatum Pers.

- fulvum $S \mathrm{~m}$.

- Iuterum Seop.

- violaceum $S m$.

Glycine Comptoniana Andr.
Glyeyrrhiza echinata $L$. foetida Desf.

glabra $\boldsymbol{L}$.

glandulifera Kit.

Gnaphalium foetidnm $L$.

Gomphrena decumbens Jacq. - globosa $L$.

Gossypium barbadense $\boldsymbol{L}$.

Grahamia aromatica Hook.

Grindelia incisa $S p r$.

Gypsophila acutifolia Fisch.

- altissima $L$

- collina Stev.

- elegans $M . B$

- repens $L$.

- Steveni Fisch.

- stricta Bungre.

Haloragis Cercodia Ait.

Hasselquistia cordata $\boldsymbol{L}$.

Hedypnois pendula $W$.

Hedysarum alpinum $L$.

- canadense $\boldsymbol{L}$.

- coronarium $L$

- flexuosum $L$

Heimia salieifolia Llc.

Helenium mexicanum Dumb.

Helianthemum angnstifolium Pers.

- racemosum Pers.

- viride Ten.

Helianthus atrorubens $L,{ }^{2}$ )

Heliopsis platyglossa Cass.

Helosciadium leptophyllum Dec.

Heraclenm angustifolium $\boldsymbol{L}$.

- asperum $M . B$.

- Panaces $L$.

- villosum Fisch

Hermannia denudata $\boldsymbol{L}$.

Herpestes chrysantha $L k$.

Heterospermum pinnatum $\mathrm{Cav}$.

Hibiscus heterophyllus Vent.

- palustris.

- radiatus Cav.

- syriacus $L$.

- trienspis Cav.

- Trionum $L$

- $\quad$ $\quad \beta$. ternatus Cav.

- " var. grandiflera.

Hieracium amplexicaule $L$.

- andryaloides Fill.

- angustifolium Hopp.

- cydonifolium Vill.

- denudatum Lapeyr.

- glaucum All.

- Lawsonii Vill.

- prenanthoides Vill.

- sabaudum $L$.

- setigerum Tauseh.

Hippocrepis unisiliquosa $L$.

Hordenm bulbosum $L$.

- coeleste Vib.

- maritimum Wither.

- nigrum $W$

- strictum Desf.

- Zeocriton $L$.

Hydrocotyle sibthorpinides Lam.

Hyoscyamus anrens $L$.

- canariebsis Ker.

Hypericum elegans $W$.

Hypochneris arachnites Biv.

Hyssopus angustifolius $M$. B.

- Foenieulnm Spr.

- nepetnides $L$.

- officinalis $\boldsymbol{L}$

Iberis amara $L$.

- pinnata $L$.

- umbellata $L$.

Impatiens parviflera $D_{e c}$.

Indigofera frutescens Thanb.

Ipomoea coceisea $\boldsymbol{L}$.

Iris halophila.

Iris maritimn Mill.

- notha $M . B$

- ochrolenca $\boldsymbol{M}$. $\boldsymbol{B}$.

- plicata Lam.

- spuria

- $-\beta . \operatorname{minor}$

- stenogyna Dec.

Isopyrum fumarioides $\boldsymbol{L}$.

Juncus tenuis $W$.

Kaulfussia amelloides Nees.

Kennedia monophylla Vent.

- prostrata $\boldsymbol{R} . B r$

- rubicunda $V$ ent.

Kitaibelia vitifolia $W$

Koautia orientalis $L$.

Kochia hyssopifolia Roth.

- scoparia Schrad.

Koeleria aegyptialea.

- Barrelieri Ten.

Lachenalia viridis $L$.

Lactuca erispa Roth.

- intybacea Jacq.

- perennis $L$.

Lapsana grandifiora $M . B$.

Laserpitium anigustifelium.

- gallicum $L$.

- pilosum $W$

Lathyrus angulatus $L$.

- annuns $L$.

- Aphaca L

- Clymenum $\boldsymbol{L}$

- latifolias $\boldsymbol{L}$.

- sativns $\boldsymbol{L}$.

- speciosus $L$

spmrius $W$.

Lavandula Iatifolia Elirh.

Lavatera arborea $L$.

- plebeja Sims.

- punetata $A I I$.

Leonnrus nepalensis.

Levisticum offieinale Knele,

Libanotis Buchtormensis Dee.

- vulgaris Dec.

Ligusticum alatum Spr.

- Seguieri Koch.

Linaria bipartita $\boldsymbol{W}$.

- geniataefolia Mill.

- litoralis Bernh.

- origanifolia $D e c$.

- porpurea Mill.

- triphylla $H$.

Lindenbergia urtienefolia Lekm.

Linum alpinum $\boldsymbol{L}$. 
Lyehnis Coeli resa Desr. - fulgens Fisch.

- Githago $\beta$. nicacensis.

Lycium afrum $L$.

Lycopersicum esculentum Mill.

Lycopus exaltatus $L$. virginicus $L$.

Lysimachia atropurpurea.

- ciliata $L$.

- verticillata Pall.

Lythrum verticillatum $L$,

Madia elegans Don.

Malaehra alceaefolia Jacq.

Malcolmia africana $\boldsymbol{R}$. $B r$. maritima $R . B r$.

Malope grandiflora Hort.

- malacoides $L$.

Malva capensis $\boldsymbol{L}$.

- erispa $L$

- lactea $L$.

- limensis $L$

- parviffora $L$.

Mariscus paniceus $\mathbf{V a h l}$.

Marrubium africanum $\boldsymbol{L}$.

- candidissimmm $L$.

Martynia proboscidea Glox.

Maurandia Barklayana Lindl.

- semperflorens, Jacq.

Meconopsis cambrica $\boldsymbol{F}_{\mathrm{ig}}$.

Medicago apienlata $W$.

- Gerardi Kit.

- glutinosa $M . B$.

- intertexta Desr.

- marginata $W$

- micraden Ehrenb.

- numumblaria Dec.

- olivaeformis Guss.

- pentacycla Dec.

- praecox Dec.

- scutellata Lam.

- Tenoriana $s p r$.

- tribuloides Desr.

- turbinata $W$.

Megastachya rigida Becuv.

Melampodinm lengifolimn Brouss.

Melica altissima $\boldsymbol{L}$.

Melilotus caerulea Desv.

var. connata.

- indica Desv.

- rotundifolia Ten.

- rugulosa $W$.

- ruthenica M. B.

Melissa alha $K$ it.

Melochia corchorifolia $L$.

Menispermum canadense $\boldsymbol{L}$

Mercurialis elliptica Lam.

Mesembrianthemum cordifolium $L$.

- erystallinum $\boldsymbol{L}$.

- glaciale Haw.

- pugioniforme $L$

Mespilus japonica Thunb.

Meum athamanticum Jacq.

Milium frntescens Sieb.

- paradoxnm L.

Mimesa Farnesiana.

- pndiea $L$.

Mimulns andicola $S_{i n}$.

- floribundas Lindl.

- Intens $L$.

- moschatus Dougl.

Mirabilis dichotoma $\boldsymbol{L}$.

- Jalappa $L$.

- longiflora $L$.

Mellia diffusa $W$.

- latifolia $W$

Moluccella laevis $L$.

Momordica Elaterinm $\boldsymbol{L}$.

Moraea chinensis Thunb.

- Sisyrinehium $\mathrm{Ker}$.

Muscari comosum $W$.
Myagrum perfoliatum $\boldsymbol{L}$.

Myosotis peduncularis Trev.

Nemophila phacelioides Bart.

Neoceis carduifolia Cass.

Nepeta coerulea Ait.

- colorata $W$.

- incana.

- italiea $L$.

- marrubioides $\boldsymbol{W}$.

- Mussini Henck.

- pannonica Jacq.

- tencrioides Lam.

- ncranica $L$.

Neslia paniculata Desv.

Nicandra physalodes Gaertn.

Nicotiana alata $L k$.

- chinensis Fisch.

- glauca Hook.

- glutinosa $L$.

- havanensis Lag.

- Langsdorffii Nees.

- macrophylla Spr.

- noctiflora Hook.

- nyctaginiflora Lehm.

- quadrivalvis Pursh.

- rustica $L$.

vincaeflora $\mathrm{Lag}$.

Nigella hispanica $L$.

- orientalis $L$.

- sativa $L$

Nolana grandiflora Horn.

- prostrata $\boldsymbol{L}$.

Nonnea vesicaria Reichb.

Ocymum polystachyum $\boldsymbol{L}$.

Oenanthe crocata $L$

- Jordani Ten.

- pimpinelluides Sm.

- virgata Poir.

Oenothera chilensis

- cruciata Nutt.

- decumbens B. Reg.

- frnticosa $L$.

- glanea $M x$.

- Lindleyana Sims.

- muricata $L$.

- odorata Jacq.

- purpurea Curt.

- Romanzovii Led.

- rosea Ait.

- Simsiana Ser.

- striata Ledeb.

- triloba Nitt.

Omphalodes linifolia Moeneh.

Onobroma eretienm Spr.

- lanatum $S p r$.

Onobrychis Capnt galli Lam.

- conferta Spr.

- Crista galli Lam.

Ononis alopecurnides $L$.

- Dehnhardtii Ten.

- fruticosa $L$.

- hircina.

- Natrix $\boldsymbol{L}$

- parviflora Thunb.

Onopordon illyrieum $\boldsymbol{L}$.

viscosum.

Ornithogalum narbonense $\boldsymbol{L}$.

Orobus atroparpureus. Desf.

- lathyroides $\boldsymbol{L}$.

- niger $\boldsymbol{L}$.

Oxybaphus Cervantesii Lag.

Paeonia humilis Retz.

- peregrina Mill.

- tenuifolia $L$.

Panicum colonum $L$.

- cuspidatum Roxb.

- virgatum $\boldsymbol{L}$.

- zonale Guss.

Papaver bracteatum Lindl.

- caucasicum M. B.
Papaver hybridum $\boldsymbol{L}$.

- nudicanle $L$.

- orientale $L$.

- somniferum $\beta$. album. spectabile $\boldsymbol{H}$. Ber.

Paspalum stoloniferum Bosc.

Passiflora edulis Sims.

- gracilis Jacq.

- heterophylla.

- Reddiana Dec.

Pastinaca pimpinellifolia $\boldsymbol{M} . \boldsymbol{B}$.

Patrinia nodiuscula Fisch.

Pavonia praemorsa $W$.

Peganum Harmala $\boldsymbol{L}$.

Pelargonium anceps Ait,

- inodorum $W$.

- malvaefolium Jacq.

- saniculaefolium $\boldsymbol{W}$

Peltaria alliacea $L$ :

Peucedanum Cervaria Lapeyr.

- involueratum Koch.

officinale $L$. verticillare Kock.

Phalaris paradoxa $L$.

Phleuin asperum Vill.

Phylica paniculata $W$.

Phyllanthus juglandifolize $\boldsymbol{W}$.

Phyllis Nobla $L$.

Physalis Alkekengi $L$.

- barbadensis Jacq.

- flexuosa $L$.

Phyteuma campanuloides $M$. $B$.

- canescens Kit.

- Schenchzeri All.

- virgatum $W$.

Phytolacca decandra $\boldsymbol{L}$

Piper pallidum Forst.

Pittosporum undulatum Andr.

Plantago Cornuti Jacq.

- Cynops L.

- eriostachya Ten.

- Kamtschatica Chan.

- sericen Kit.

- sibiriea Poir.

- stricta Schousb.

Plumbago coerulea Humb.

- micrantha Desf.

- zeylanica $L$.

Poa badensis $W^{T}$.

- Molineri Balb.

- pumila.

- purpurascens Spr.

Pocockia cretica Ner.

Podalyria australis $W$.

Polemoninm coeruleam $\boldsymbol{W}$.

- mexicanum Cern

Polyenemum Henffelii Lang.

Polygonum cymosum Trev.

- emarginatmm Roth

Potentilla atrosanguinea Don.

- calabra Ten.

- canescens.

- collina $W^{\prime} i b$.

- formesa Don.

- genides M. B.

- Güntheri.

- reeta $L$.

- rupestris $L$.

Poterium garganienm $\boldsymbol{T o n}_{\mathrm{n}}$

- Polygamun Kit.

- spinosnm $L$.

Pothos maerophyllue Suo.

Prismatocarpos falcatus Ten.

Prunella alba Pall.

- grandiflora $L$.

- hyssopifolia $L$.

- laciniata.

- pensylvanica $W$.

Proralea bituminosa $L$,

- hirta $L$.

Psoralea pinnata $\boldsymbol{L}$. - verrucosia $W$.

Pulicaria arabica.

Pycnanthemum virginicum Pers.

Pyrethrum corymbesum $W$.

- indicum Roxb.

- macrophyllum $W$.

- millefoliatum $W$.

- pulverulentum $\boldsymbol{W}$. tenuifolium $T e n$.

Queria hispanica $W$.

Ranunculus caucasicus $M$. B.

- cortusaefolius $W$.

- muricatus $L$.

- pedatug $K i t$.

- Stevenii Bess.

- tuberculatus Dec.

Reseda abyssinica Fres, ${ }^{3}$ )

- alba $L$.

- crispata $L k$.

- Phytenma E.

Rhagadiolus edulis Gaertn.

- Koelpinia $W$.

- stellatus $W$.

Rheum cohpactum $\mathbf{L}$.

- crispum.

- Rhaponticam $L$

- sibiricum.

- talaricum $\boldsymbol{L}$.

- tauricam.

- undulatum $\boldsymbol{L}$.

Ribes aureum Pursh.

Ricinus communis $L$.

Rivina laevis $L$.

- purpurascens Sehrad.

Rosa pimpinellifolia carnea. rubifolia $R$. $B r$.

Rudbeckia amplexifolia Jacq.

- laciniata $L$.

- triloba $L$.

Rumex alpinus $\boldsymbol{L}$.

- aquaticus $L$.

- confertus $W$.

- cristatus Dec.

- domesticns Hartm.

- laevigatns.

- maximus Schreb.

- nemorasus Schrad.

- Patientia $L$.

- pratensis Koch.

- pulcher $L$.

- purpureus Poir. 
Saxifraga pyramidalis Lap.

- rotundifolia $L$.

- serrata Ott.

- spathulata Desf.

Scabiosa africana $L$.

- alpina $L$.

- atropurpurea $\boldsymbol{L}$.

- australis Wulf.

- caucasica $M . B$.

- centauroides Lam.

- corniculata Kit.

- graminifolia $L$.

- Hadnikiana Host.

- stellata $\boldsymbol{L}$

- uralensis Murr.

Scandix brachycarpa Guss.

Schismns marginatus $P$. $B$.

Schivereckia podolica Andrz.

Schizanthus porrigens Hook.

Scoparia duleis $L$.

Scorpiurus muricata $L$.

- suleata $L$.

- vermiculata $L$.

Scrofularia Balbisii Horn.

- cordata Pers.

- glandulosa Kit.

- peregrina $\boldsymbol{L}$.

- rupestris $\boldsymbol{M}$. $B$.

- sambucifolia $\boldsymbol{L}$.

- variegata $M . B$.

- vernalis $\boldsymbol{L}$.

Scutellaria alpina $\boldsymbol{L}$.

- lateriflora $\boldsymbol{L}$.

- pallida $\boldsymbol{M}$. $\boldsymbol{B}$.

- peregrina $L$.

- rubicunda Horn.

Secale fragile $M$. $B$.

Sedum lividum $W$.

- Rhodiola Dec.

Senecio Doria $\boldsymbol{L}$.

- elegans $L$.

- ovatus $W$.

Seriola aethnensis $\boldsymbol{L}$.

Serratula alata $W$.

- cynaroides Dee.

- radiata $\boldsymbol{M}$. $\boldsymbol{B}$.

Seseli glancum $\boldsymbol{L}$.

- gummiferum $S \mathrm{~m}$.

- montanum $\boldsymbol{L}$.

- rigidum Kit.

- tortuesum $L$.

- varium Trev.

Setaria italica $\boldsymbol{P}, \boldsymbol{B}$.

- Weinmanni R. Sch.

Sida Abutilon $\boldsymbol{L}$.

- angustifolia Juss.

- mollissima.

- Napaea Cav.

- triloba Cav.

- vesicaria Cav.
Sideritis montana $\boldsymbol{L}$.

Silene alpestris Jacq.

- Atocion Jacq.

- chlerantha Ehrh.

- compacta Horn.

- cretica $L$.

- flavescens Kit.

- fruticosa $L$.

- gallica $L$.

- hirsuta Lag.

- imbrieata Desf.

- inamoena Lindl.

- infracta Kit.

- linaccola Wib.

- livida $W$.

- mollissima $S m$.

- pendula $L$

- quinquevulnera $L$.

- saponariaefolia Schott.

- sericea $A l l$.

- viridiflora $L$.

Sinapis auriculata Dec.

- turgida Delil.

Sisymbrium anstriacum Jacq

- strictissimum $L$.

Sisyrinchium Bermudiana $L$.

- laxum $L k$.

- striatum Cav.

Solanum astroides Jacq.

- campechiense $L$.

- corymbosum Jacq.

- flavum Ait.

- guineense.

- humile.

- marginatum $L$

- ovigerum Dun.

- Pseudocapsicum L

- radicans $L$.

- sodomeum $\boldsymbol{L}$.

- Zuccagnianum Dur.

Sonchus flexuosus Ledeb.

- fruticosus.

- leucophaeus $W$.

palustris $L$.

Sorghum bicolor $W$.

- caffrorum.

- saccharatum Pers.

- vulgare v. pyramidatum.

Spartina cynosuroides Roth.

Spartium junceum $\boldsymbol{L}$.

Spilanthes oleracea $L$.

Stachys alpina $\boldsymbol{L}$.

- excelsa $\boldsymbol{H}$. Par.

- iberica M. B.

- polystachya Ten.

- purpurea Poir.

Stachytarpheta jamaicensis $V a h l$.

Statice latifolia $S_{m}$.

- Limonium $L$.

- mucronata $L$.
Statice palmaris $S m$.

- sinuata $L$.

Stipa capillata $I$

- gigantea Lag.

Stylidium adnatum $\boldsymbol{R}$. $\boldsymbol{B r}$.

- fruticosum $R . B$ r.

Symphoria racemosa Pursh.

Syrenia Biebersteinii Andrz.

Tagetes erecta $L$. patula $L$.

Talinum purpurenm Fisch.

Teedia Incida- $R$ ud.

Tellima grandiflora Lindl.

Teucrium flavum $\boldsymbol{L}$.

- hyrcanicum $\boldsymbol{L}$.

- orientale $L$.

- Pseudo-Scorodonia Desf.

Thrincia hirta Roth.

Thymus canus Stev.

- Crantzianus H. Genev.

- grandiflorus.

- neglectus $\boldsymbol{H}$. Genev.

- Nepeta Scop.

Tiaridium indicum Lehm.

Tolpis barbata Gaertn.

Tordylium apulum Riv. maximum $\boldsymbol{L}$. syriacum $\boldsymbol{L}$.

Trachelium coeruleum $L$.

Tradescantia erecta Cav.

- Selloviana Pohl.

Tragopogon campestria Bess.

- floccosus Kit. porrifolins $\boldsymbol{L}$

Tribulus terrestris $\boldsymbol{L}$.

Trifolium elegans Sav.

- incarnatum $L$.

- libanoticum Ehrenb.

- ligusticum Balb.

- subterraneum $\boldsymbol{L}$.

- vesiculosum Sant.

Trigonella areuata Meyer.

- Calliceras Fisch.

- cancellata Fisch.

- Foenum graecum $L$.

- tenuis Fisch.

- uralensis Led.

Trinia vulgaris Dec.

Triodia cuprea Jacq.

Triticum amylenm Ser.

- Banhini Lag.

- Cienfuegos Lag.

- eristatum Schreb.

- dasyanthon Ledeb.

- desertorum Fisch.

- dicoccon Schuebl.

- hordeiforme Host.

- junceum $\boldsymbol{L}$.

- monococcum $\boldsymbol{L}$.
Triticum polonicum $L$.

- rigidum Schrad.

- tricoccum Schuebl. turgidum $L$.

Trimmfetta oblongata $\boldsymbol{L} k$. triclada $L k$.

Trollius caucasicus Stev.

Urospermum Dalechampii Desf. picroides Desf.

Urtica cannabina $\boldsymbol{L}$.

- convexa Horn.

Valerianella coronata $D_{e o}$.

- tridentata $\boldsymbol{L} k$.

- uncinata Dufr.

Verbascum australe Schrad.

- Blattaria $\boldsymbol{L}$.

- blattarioides Lam.

- condensatum Schrad.

- orientale $\boldsymbol{M}$. $\boldsymbol{B}$.

- rotundifolium $T e n$.

Verbena bonariensis $\boldsymbol{L}$.

- Bracteosa $M x$.

- hastata $\boldsymbol{L}$.

- lasiostachys $L k$.

- paniculata Lam.

- urticaefolia $L$.

- venosa.

Vernonia anthelminthica $W$

Veronica anstralis Schrad.

- biloba Vaht.

- brevifolia $M$. $\boldsymbol{B}$.

- caucasica $\boldsymbol{M}$. $\boldsymbol{B}$.

- gentianoides $\boldsymbol{V a h l}$.

- sibirica $L$.

- virginica $L$.

Vesicaria cretiea Poir.

- sinuata Poír.

- utriculata Lam.

Vicia alba Moench.

- articulata $W$.

- atropurpurea Desf.

- biennis $L$.

- bithynica $\boldsymbol{L}$.

- dasycarpa Ten.

- globosa Retz.

- Iutea $L$.

- Nissoliana Gouan.

- platycarpa.

- tenuissima $\boldsymbol{H}$. Ber.

Xanthium orientale $\boldsymbol{L}$.

- spinosum $L$.

Xeranthemun annnum $L$.

- cylindricum $\$ \mathbf{S m}$.

- erectnm.

Ximenesia encelioides Cav.

Zinnia elegans vair. coccinea.

- $"$ violacen.

Ziziphora dasyantha $M$. $B$.

Zoegea Leptaurea $\boldsymbol{L}$.

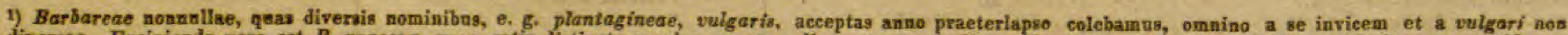

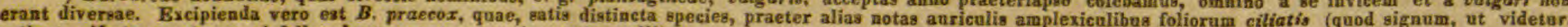
satis manifestum autores, ni fallor, hucusque praetermiserunt) excellit.

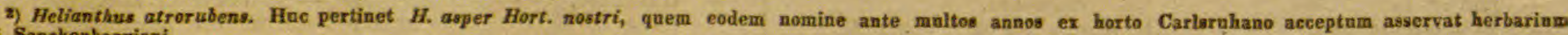
musei Senckenbergiani.

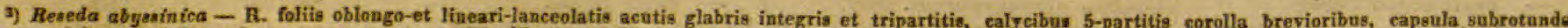

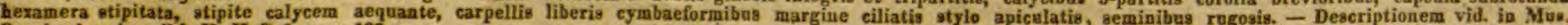
Seackenbergian, Bd. II. Heft 1. pag. 106. 


\section{Semina in horto botanico Francofurtensi anno 1836 collecta.}

Acaena eylindrostachys $R$. et $P$. - sericea Jacq.

Achillea Ageratum $\boldsymbol{L}$.

- odorata $L$.

Achyranthes axillaris $W$.

- brachiata $\boldsymbol{L}$.

Achyropappus schkuhrioides $\boldsymbol{L} k$.

Aconitum Anthora $L$.

- neubergense $D e c$.

- volubile Pall.

Acroglochin chenopodioides Schrd.

Actaea brachypetala Dec.

- racemosa $L$

Actinomeris oppesitifolia Fres. ${ }^{1}$ )

Adenocarpus foliolosus Dec.

Aegilops cylindrica Host.

- ovata $L$

- squarrosa $L$.

- triuncialis $L$

Agropyrum scirpeum Guss.

Agrostis versicolor Stev.

Ajuga Chamaepitys Schreb.

Allionia nyctaginea Mchx.

Allium acutangulum $\boldsymbol{W}$.

- albidum Fisch.

- baicalense $\boldsymbol{W}$.

- cernuum Roth.

- fistulosum $\boldsymbol{L}$.

- fragrans Vent.

- neapolitanum Cyrill.

- Ophioscorodon Link.

- ramosum Jacq.

- senesceus $L$.

- sibiricum $L$.

- striatum Jacq.

- tenuiflornm Ten.

- triquetrum $L$

- Vietorialis $\boldsymbol{L}$.

Alstroemeria Pelegrina $\boldsymbol{L}$.

Althaea cannabina $L$.

- Froloviana Fisch.

- Sieberi Lk..

Amethystea coerulea $\boldsymbol{L}$.

Ammannia latifolia $\boldsymbol{L}$.

Ammi majus $L$.

- Visnaga Lam.

Aromobium alatum $R$. $\mathrm{Br}$.

Anacyclus Pyrethrum Lk.

Anchusa asperrima Delil.

- hybrida Ten.

- paniculatis Ait.

- rosea $M . B$.

- sempervirens $\boldsymbol{L}$.

Androsace lactiflora Fisch.

- maxima $L$.

- septentrionalis $\boldsymbol{L}$.

Androsaemum officinale All.

Andryala cheiranthifolia Herit,

- lanata $L$.

Anemone Hudsoniana Richards.

- ochotensis Fisch.

Angelica decurrens Led.

Anisodus luridus Link.

Anoda hastata Cav.

Anthemis psorosperma Ten.

- rigescens $W$.

- ruthenica $M . B$.

- secundiramea Biv.

Anthephora elegans Schreb,

Anthyllis tetraphylla $L_{\text {. }}$.

Aquilegia glandulosa Fisch.

- sibirica Lam.

- speciosa H. Marb.

Arabis alpina $L$.
Arabis hirsuta Scop.

- lilacina Schrad.

- oxyota Dec.

- pendula L.

- Turrita $L$.

Ardisia crenulata $V$ ent.

Arenaria calycina Poir.

- rigida $M$. $B$.

- rostrata Kit.

Argemone Barklayana Grah.

- mexicana $\boldsymbol{L}$. $\beta$. albiflora.

Artemisia violacea Desf.

Asclepias angustifolia $W$.

- curassavica $L$.

- incarnata $\boldsymbol{L}$.

- nivea $L$.

Asphodelus albus $W$.

- clavatus Roxb.

- creticus Lam.

- Inteus L.

- tanricus $M . B$.

Asprella major Fresen. ${ }^{2}$ )

Astragalus alopecuroides $L$

- asper Jacq.

- baeticus $L$.

- brachyceras Led.

- carolinianus $L$.

- Cicer $\boldsymbol{L}$.

- galegiformis $\boldsymbol{L}$.

- pentaglottis $\boldsymbol{L}$

- subulatus Pall.

- sulcatus $L$

- tribuloides $\boldsymbol{H}$. Berol.

Athamanta flexuosa Desf.

- Matthioli $W u l f$.

Atheropogon apludoides $W$.

Anbrietia Columnae Guss. deltoidea Dec.

Avena planiculmis Schrad. rigida $M . B$.

Baccharis ivaefolia $\boldsymbol{L}$.

Balbisia elongata $W$.

Barbarea praecox $R$. $B r$.

Barkhausia alpina $L k$.

- graveolens Rchb.

- leontodontoides Rchb. rubra $L k$.

Basella rubra $L$.

Beta foliosa Ehrenb.

Betonica incana Ait.

- orientalis $\boldsymbol{L}$.

Bidens bipinnata $L$.

- grandiflora Balb.

- lencantha $W$.

Billardiera scandens $S \mathrm{~m}$.

Biscutella apula $L$.

- eriocarpa Dec.

- lyrata $\boldsymbol{L}$.

- maritima Ten.

Biserrula Pelecinns $L$

Blumenbachia insignis Schrad.

Boebera glandulosa Pers.

Brachypodinm asperum $R$. et Sch. - longifolium $P . B$.

Briza maxima $L$.

Brizopyrum siculum $L k$,

Bulbine annua $W$.

Bunium peucedanoides $M$. $B$.

Buphthalmum salicifolium $\boldsymbol{L}$.

- spinosum $\boldsymbol{L}$.

Buplenrum altaieum Led.

- faleatum $\boldsymbol{L}$.

- heterophyllum $L k$.
Bupleurum multinerve Dec. - nervosum Trev.

- protractum $L k$. sibiricum Trev.

Cacalia sonchifolia $\boldsymbol{L}$.

Cakile perennis Dec. rugosa $D e c$.

Calamintha rupestris Host.

Calandrinia compressa Schrad.

- discolor Schrad.

- grandiflora Lindl.

Caldasia heterophylla $W$.

Calendula aegyptiaca Desf. pluvialis $L$.

- stellata Cav.

Calfistachys lanceolata $V$ ent.

Camelina dentata Pers.

Camellia japonica $L$.

Campanula betonicaefolia $M$. B.

- divergens $W$.

- hybrida $L$.

- ilinoensis Fresen, ${ }^{3}$ )

- obliquifolia Ten.

- peregrina $L$.

- pyramidalis $L$

- silbirica $L$.

- stylosa Lam.

- urticaefolia Schmidt.

Canna speciosa Rose.

Capraria biflora $L$

Capsicum bicolor Jacq.

- ciliare $W_{\text {. }}$

pendulum $W$.

Cardiospermum Halicacabum $\boldsymbol{L}$.

Carduus Argyroa Biv.

Carthamus tinctorius $\boldsymbol{L}$.

Carum Bulbocastanum Koch.

Cassinia spectabilis $R, B r$.

Catananche coerulea $L$.

Caucalis grandiflora $\boldsymbol{L}$.

- platycarpos $\boldsymbol{L}$.

Ceanothus coerulens

Celosia castrensis $L$.

- cristata $\boldsymbol{L}$.

- margaritacea $L$ virgata Jacq.

Celsia Areturus $L$.

- australis.

- lanata Jacq.

Cenchrus echinatus $L$

Centaurea calocephala $w$.

- cientaefolia Horn.

- coriacea Kit.

- Crupina $L$.

- depressa M. $B$.

- eriophora $L$.

- melitensis $L$.

- moschata $L$

- rupestris $\boldsymbol{L}$.

- ruthenica Lam.

- sonchifolia $L$.

- stereophylla Bess.

- sulphurea $W$.

Centrachne viscida Schott.

Centrospermum Chrysanthemum

Cerastinm davmricume

- grandiflorum Kit.

- Ledebourianum.

Ceratochloa pendula Schrad.

- unioloides Dec.

Chaerophyllum trichospermum.

Chaptalia Anandria Spr.

Cheirinia crepidifolia $L k$.

Chelidoninm laciniatum Mill.

Chelone campanulata Cav.

- conferta $R \cdot B r$

- diffusa Dougl.

- Digitalis $S p r$

- glantulosa.

- hirsuta $L$

- ovata Dougl.

Chenopodium ambrosioides $\boldsymbol{L}$.

- Botrys L.

- chilense Schrad.

- foetidum.

Chorizema rhombenm $R . B r$.

Chryseis tenniflora $\boldsymbol{H}$. Jen.

Cicer arietinnm $L$.

Cineraria canadensis $L$.

Cirsium lanifínrum $M$. $B$

- scleranthon $M . B$.

- silyboides.

Cistus monspeliensis $L$

- vaginatus Ait.

Clarkia elegans Dougl.

- pulchella Pursh.

Claytonia perfoliata $D_{0}$.

Clematis angustifolia Jacq

Cleome graveolens Rafin.

- spinosa $L$.

- violacea $L$

Cneorum tricoccon $L$

Cnicus spurius $H o f f m$.

- syriacus $W$.

Cnidium apioides Spr.

- cuneatum Led.

Coix exaltata Jacq.

Collomia coccinea Lehm.

- grandiflora Dougl.

Commelina angustifolia $M$

- clandestina Mart.

- polygama Roth.

Conium maculatum $\beta$. sibiricum

Conringia perfoliata $L k$.

Convolvulas siculus $L$.

Coreopsis Atkinsoniana Dougl. lanceolata $L$

Coriaria myrtifolia $\boldsymbol{L}$.

Corispermum canescens Kit.

Coronilla coronata $L$.

- glauea $L$.

- Secnridaca $L$.

Corydalis aurea $W$.

- sempervirens Pers.

Cosmea bipinnata $W$.

Crambe maritima $\boldsymbol{L}$.

Crncianella angustifolia $L$

Cucubalus bacriferus $L$.

Cucumis prophetarum $L$,

Cucurbita lagenaria $L$.

Cuphea procumbens Cav.

- silenoides Nees.

- viscosissima Jaeq.

Cyclachaena xanthifolia $F$

Cynanehnm nigrum Pers.

Cynoglnssum apenninum $\boldsymbol{L}$.

- bicolor $W$

- canescens $W$.

- cheirifolium.

- Dioscoridis Vill.

- gylvaticum Sm.

Cyperus alternifolins $L$. 
Datura Metel $\boldsymbol{L}$. - Tatula $L$.

Daucus gummifer Lam. - hispidus Desf.

- muricatus $\boldsymbol{L}$.

Delphinium grandiflorum $L$. - Requienji Dec.

Desmanthus virgatus $\boldsymbol{W}$.

Dianthus collinus Kit.

- diutinus Kit.

- sylvaticus Hopp,

- velutinus Guss.

- virgineus $L$.

Digitalis australis Ten.

- Buxbaumii Bess.

- ferruginea $\boldsymbol{L}$.

- grandiflora Lam.

- laevigata Kit.

- Ianata Ehrh.

- lutea $L$.

Digitaria aegyptiaca $\boldsymbol{W}$.

Diplachne fascicularis $P$. $B$.

Dolichos brasiliensis.

- Catiang $\boldsymbol{L}$.

- Lablab $L$.

- melanophthalmos Dec.

Doronicum mexicanum.

Draba aurea $V a h l$.

Dracocephalum canariense $\boldsymbol{L}$.

- canescens $\boldsymbol{L}$.

- Moldavica $L$.

- nutans $\boldsymbol{L}$.

- peltatum $\boldsymbol{L}$.

- sibiricum $\boldsymbol{L}$.

Eccremocarpus scaber $\boldsymbol{R}$. et $\boldsymbol{P}$.

Echinops Ritro $\boldsymbol{L}$.

- sphaerocephalus $\boldsymbol{L}$.

Echinospermum virginicum. ${ }^{\text {s) }}$

Echium creticum $L$.

- grandiflorum Desf.

- italicum $\boldsymbol{L}$.

- salmanticum Lag.

Eclipta erecta $\boldsymbol{L}$.

- latifolia $L$.

- prostrata $L$.

Elensine coracana Pers.

- indica $L a m$.

- rigida $S p r$.

- Tocussa Fres.

Elichrysum bracteatum Vent. - fuIgidum $W$.

Elsholtzia cristata $\boldsymbol{W}$.

Elymus arenarius $\boldsymbol{L}$.

- canadensis $\boldsymbol{L}$.

- carolinianus.

- giganteus Vahl.

- Hystrix $L$.

- propinquus Fresen. 9

Emex spinosus Camp.

Entelea arborescens $\boldsymbol{R}$. Br.

Epilebium angustissimnm Ait.

Eragrostís abyssinica $\boldsymbol{L k}$.

Eranthis hyemalis Salisb.

Erigeron contortus Desf.

- elongatus Led.

- podelieus Bess.

- Villarsii Bell.

Erinus alpinus $\boldsymbol{L}$.

Erodinm gruinum Ait.

- moschatum Ait.

- pulverulentum $W$.

Ervum agrigentinum Guss.

- Ervikia

Eryngium giganteum $M$. B. - planum $L$.

Erysimum altaicum Meyer.

Eschscholzia ealifornica Cham.

Ethulia conyzoides $\boldsymbol{L}$.

Eupatorium ageratoides $\boldsymbol{L}$. - album $\boldsymbol{L}$.
Euphorbia cyathophora Jacq. - Humboldtii $W$.

- Lagascae Spr. prunifolia Jacq.

Exacum viscosum $S m$.

Farsetia clypeata $\boldsymbol{R}, \boldsymbol{B r}$.

- eriocarpa Dec.

Fedia Cornucopiae Vahl.

Flaveria repanda $\mathrm{Lag}$.

Forskolea angustifolia Retz.

- tenacissima $L$.

- viridis Ehrenb.

Fragaria indica $A n d r$.

Francoa sonchifolia $\boldsymbol{S} p r$.

Fuchsia arborescens Sess.

Fumaria capreolata $\boldsymbol{L}$.

Galardia bicolor Lam.

Galega offieinalis $\boldsymbol{L}$.

- persica Pers.

- strieta Ait.

Galinsogea trilobata Cav.

Galinm infestum Kit.

- lncidum.

- spurium.

- verrucosum $\boldsymbol{S}$.

Garidella Nigellastrum $\boldsymbol{L}$.

Gaura biennis $L$.

- tripetala Cav.

Genista canariensis $\boldsymbol{L}$.

- hispanica $L$.

- virgata $W$.

Gentiana cruciata $\boldsymbol{L}$.

Geranium carolinianum $\boldsymbol{L}$.

- sanguineum $\boldsymbol{L}$.

- sibiricum $\boldsymbol{L}$.

Geropogon glaber $\boldsymbol{L}$.

Geum albuin Gmel.

- atlanticum. Desf.

- chilense Balb.

- coccineum Sm.

- heterophyllum.

- ranunculoides Ser.

Gilia achilleaefolia Benth.

- inconspicua Hook.

- laciniata $R$, et $P$

Gladiolus psittacinus.

Glaucium corniculatum Pers.

- fulvum $S m$.

- luteum Seop.

- violaceum Sm.

Glycine Comptoniana Andr.

Glycyrrhiza echinata $\boldsymbol{L}$. foetida Desf.

glabra $\boldsymbol{L}$.

glandulifera Kit.

Gnaphalium foetidum $\boldsymbol{L}$.

Gompholobium tomentosum $\mathbf{L a b}$.

Gomphrena decumbens Jacq. globosa $\boldsymbol{L}$

Grahamia aromatica Hook.

Grewia occidentalis $\boldsymbol{L}$.

Grindelia incisa Spr.

Gypsophila acutifolia Fisch.

- altissima $\boldsymbol{L}$.

- collina Stev.

- elegans $\boldsymbol{M}$. $\boldsymbol{B}$.

- repens $\boldsymbol{L}$.

- Steveni Fiseh.

strieta Bunge.

Haloragis Cercodia Ait.

Fasselquistia cordata $L$.

Hedypnois pendula $W$.

Hedysarum alpinnm $\boldsymbol{L}$.

- canadense $\boldsymbol{L}$.

- coronarium $L$.

- flexunsum $\boldsymbol{L}$. sibiricum Lam.

Heimia salicifolia $L k$.

Helenium autumnale $\boldsymbol{L}$.

- mexieanum Humb.
Helianthemum aegyptiacum Mill. | Laetnea erispa Roth.

- racemosum Pers.

Helianthus lenticularis Lindl.

Heliopsis platyglossa Cass.

Helosciadium leptophyllum Dec.

Heracleum angustifolium $L$.

- asperum M. $B$.

- Panaces $L$.

- speciosum.

villosum Fiseh.

Heterospermum pinnatum Cav. Heterotheca inuloides Cass.

Hibisens palustris.

- radiatus Cav.

- syriacus $L$.

- Trionum $L$

" $\beta$. ternatus Cav.

var. grandiflora.

Hieracium amplexieaule $L$.

- andryaloides Vill.

- angustifolium Hopp.

- ceratophyllum Led.

- corymbesum Led.

- cydonifolium Vill.

- denudatum Lapeyr.

- glaucum All.

- Lawsonii Vill.

- prenanthoides Vill.

- sabaudum $L$.

Hippoerepis unisiliquosa $L$.

Hordenm bulbosum $\boldsymbol{L}$.

- coeleste Vib.

- maritimum Wither.

- nigrum $W$

nudum.

- strictum Desf.

- Zeocriton $\boldsymbol{L}$.

Horminum eanlescens Orteg.

Hyoscyamus aureus $L$.

- canariensis $K e r$.

Hypericum elegans $W$.

- veronense.

Hypoehoeris arachnites Biv.

Hyssopus angustifolius $M$. $B$.

- Foeniculum Spr.

- Lophanthus $L$

- nepetoides $L$.

- officinalis $\boldsymbol{L}$

- orientalis $W$.

Impatiens parviflora Dec.

Indigofera australis $\boldsymbol{W}$.

Inula bifrons $\boldsymbol{L}$.

- suaveolens Jacq.

Ipomoea coccinea $\boldsymbol{L}$.

Ipomopsis elegans $M x$.

Iris halophila.

- Güldenstädtiana Lepeeh.

- maritima Mill.

- mauritiana.

- ochroleuca M. B.

- plicata Lam.

- spuria.

- - B. minor.

- stenogyna Dec.

Isopyrum fumarioides $\boldsymbol{L}$.

Juncus tenuis $\boldsymbol{W}$.

Kaulfussia amelloides Nees.

Kennedia apetala Lodd.

- monophylla Vent.

- prostrata $R \cdot B r$.

- rubicunda Vent.

Kitaibelia vitifolia $w$.

Kleinia suffruticosa $W$.

Knantia orientalis $\boldsymbol{L}$.

Kochia hyssopifolia Roth.

- seoparia Schrad.

Koeleria aegyptiaca.

- glauea Dec.

- laxa $L k$.

Lachenalia viridis $L$.

- intybacea Jacq.

- perennis $L$.

Lamarckia aurea Mönch.

Lapsana grandiflora $\boldsymbol{M}$. $\boldsymbol{B}$.

Laserpitium angustifolium.

- gallicum $L$.

- pilosum $\boldsymbol{W}$.

- pruthenicum $\boldsymbol{L}$.

Lathyrus angulatus $\boldsymbol{L}$.

- annuus $\boldsymbol{L}$.

- Aphaca $L$.

- Clymenum $\boldsymbol{L}$.

- latifolius $\boldsymbol{L}$.

- sativus $\boldsymbol{L}$.

- speciosus $\boldsymbol{L}$.

- spurius $W$.

Lavandula latifolia Ehrh.

Lavatera arborea $\boldsymbol{L}$.

- plebeja Sims.

- punctata All.

Leonurus lacerus Lindl.

- nepalensis.

Levisticum officinale Koch,

- vulgaris Dec.

Ligusticum alatum Spr.

- Segnieri Koeh.

Linaria bipartita $W$.

- genistaefolia Mill.

- litoralis Bernh.

- origanifolia Dec.

- purpurea Mill.

- triphylla $\boldsymbol{W}$.

Linum alpinum $\boldsymbol{L}$.

- narbonense $\boldsymbol{L}$.

- suffruticosum $\boldsymbol{L}$.

Lobelia alata $R$. $B r$.

- coronopifolia $\boldsymbol{L}$

- triquetra $L$.

Lotus edulis $\boldsymbol{L}$.

- glaberrimus Dec.

- Jacobaeus L.

- ornithopedioides $L$.

- Requieni Maur.

- siliquosus $\boldsymbol{L}$.

Lupinus albus $\boldsymbol{L}$.

- angustifolius $\boldsymbol{L}$.

Lasiospermum radiatum Trev

- longipedunculatus Led.

Libanotis Buchtormensis Dec.

Lindenbergia urticaefolia $\mathrm{Lehm}$.

Loasa bryoniaefolia Schrad.

Lophospermum scandens Don. 
Malva lactea $\boldsymbol{L}$.

- limensis $L$.

- parviflora $L$.

Marrnbium candidissimm $\boldsymbol{L}$.

Martynia proboscidea Glox.

Maurandia Barklayana Lindl.

- semperflorens Jacq.

Meconopsis cambrica Vig.

Medicage apiculata $W$.

- Gerardi Kit.

- glutinosa M. B.

- intertexta Desr.

- marginata $W$.

- microdon Ehrenb.

- nummularia Deo.

- olivaeformis Guss.

- pentacycla Dec.

- praecox Dee.

- scutellata Lam.

- Tenoriana Spr.

- tribuloides Desr.

- turbinata $\boldsymbol{W}$

Megastachya rigida Beauv.

Melampodium longifolium Brouss.

Melica altissima $\boldsymbol{L}$

Melilotus caerulea Desv.

- " var. connata.

- indica Desv.

- rotundifolia Ten.

- rugulosa $\boldsymbol{W}$.

- ruthenica $M$. $B$.

Melissa alba Kit.

Melochia corchorifolia $\boldsymbol{L}$.

Menispermum canadense $\boldsymbol{L}$.

Mercurialis elliptica Lam.

Mesembrianthemum cordifolium L.

- erystallinum $L$. pugieniforme $\boldsymbol{L}$.

Meum athamanticum Jaeq.

Mimosa Farnesiana. pudica $\boldsymbol{L}$.

Mimulus andicola $\boldsymbol{S} m$.

- floribundus Lindl.

- luteas $L$.

- moschatus Dougl.

Mirabilis dichotoma $\boldsymbol{L}$.

- Jalappa L.

- lengiflora $L$

Mollia diffusa $\boldsymbol{W}$

- latifolia $\boldsymbol{W}$

Moluccella laevis $\boldsymbol{L}$.

Momordica Elaterium $\boldsymbol{L}$.

Moraea chinensis Thunb.

Morina persica $L$.

Muscari comesum $W$.

Myagrum perfoliatum $\boldsymbol{L}$.

Nemophila phacelisides Bart.

Neoceis carduifolia Cass.

Nepeta coerulea Ait.

- colerata $W$.

- grandiflora.

- ineana.

- italica $L$

- longiflora Vent.

- marrubioides $W$.

- pannonica Jacq.

- teucrioides Lam.

- ueraniea $L$.

Neslia pamiculata Desv.

Nieandra physalodes Gaertn.

Nicotiana chinensis Fisch.

- glauea Hook.

- glntinosa $L$.

- havanensis Lag.

- Langsdorffii Nees.

- maerophylla Spr.

- noctiflora Hook.

- nyctaginiflora Lehm.

- quadrivalvis Pursh.

- rustica $L$.
Nicotiana vincaeflora Lag.

Nigella hispanica $L$. orientalis $L$ sativa $\boldsymbol{L}$.

Nolana grandiflora Horn. - prostrata $L$.

Ocymum polystachynm $L$.

Oenanthe crocata $\boldsymbol{L}$. - pimpinelloides $S m$.

- virgata Poir.

Oenothera ehilensis

- cruciata Nutt.

- decumbens B. Reg.

- fruticosa $L$.

- glauca $M x$

- Lindleyana Sims.

- muricata $\boldsymbol{L}$.

- odorata Jacq.

- purpurea Curt.

- Romanzovii Led.

rosea Ait.

- Simsiana Ser.

- striata Ledeb.

- tetraptera Cav.

- triloba Nutt. undulata.

Omphalodes Iinifolia Moench.

Onobroma cretieum Spr.

- lanatum Spr.

Onobryehis Caput galli Lam.

- conferta $s p r$.

- Crista galli Lam.

Ononis alopecuroides $L$.

- fruticosa $L$.

- hircina.

- Natrix $L$

- parviflora Thunb.

Ornithogalum abyssinicum Fres.

- narbonense $L$.

Orobus atropurpureus Desf.

- lathyroides $L$. niger $L$.

Paeonia humilis Retz.

- peregrina Mill.

- tenuifolia $L$

- villosa.

Panicum colonum $\boldsymbol{L}$.

- virgatum $\boldsymbol{L}$.

- zonale Guss.

Papaver bracteatum Lindl.

- cancasicum M. B.

- hybridum $L$.

- nudicanle $L$.

- orientale $\boldsymbol{L}$.

- somniferum $\beta$. album.

- spectabile $\boldsymbol{H}$. Ber.

Paspalum stoloniferum Bose.

Passiflera edulis Sims.

- gracilis Jacq.

- heterophylla.

- Raddiana Dee.

Pastinaea pinpinellifolia M. B.

Pavonia praemorsa $W$.

Peganum Harmala $L$.

Pelargonium anceps Ait.

- inodorum $w$.

- malvaefolinm Jacq.

- saniculaefolium $W$.

Peucedanum involucratam Kech. - verticillare Koch.

Phalaris paradoxa $\boldsymbol{L}$.

Phleum asperum Vill.

Phlomis tuberosa $\boldsymbol{L}$.

Phylica paniculata $W$.

Phyllanthus juglandifolins $\boldsymbol{W}$.

Phyllis Nobla $L$.

Physalis Alkekengi $L$.

- flexuesa $L$.

Phyteuma canescens Kit.

- Scheuchzeri $\mathbf{A l l}$.
Phyteuma strictum Ker.

- virgatum $W$

Phytolacca deeandra $L$.

- icosandra $L$.

Pimelea drupacea Lab.

Pimpinella aromatica $\boldsymbol{M}$. $\boldsymbol{B}$.

- peregrina $L$.

Piper pallidum Forst.

Pittosporum undulatum $A n d r$.

Plumbago micrantha Desf.

Pocockia cretica Ser.

Podalyria australis $W$.

Polemoninm coerulerim $W$.

- mexicanum Cerv.

Polygonum cymosum Trev. emarginatum Roth.

Potentilla arguta Pursh.

- calabra Ten.

- canescens.

- collina Wih.

- formesa Don.

- geoides M. B.

- gracilis Dougl.

- Güntheri.

- recta $L$.

rupestris $L$.

Poterium garganicum Ten.

- polygamum Kit.

- spinosum $\boldsymbol{L}$.

Prismatocarpos falcatus Ten.

Prunella alba Pall.

- grandiflora $L$.

- hyssopifolia $\boldsymbol{L}$.

- laciniata.

- pensylvanica $W$.

Psoralea bítuminesa $L$

- capitata Thunb.

- hirta $L$.

- pinnata $L$

- verrucosa $W$.

Pycnanthemum virginieum Pers.

Pyrethrum corymbosum $\boldsymbol{W}$.

- indicus Roxb.

- macrophyllun $W$.

- millefoliatum $W$. tenuifolium Ten.

Qneria hispanica $W$.

Ranunculus caucasicus $M$. B.

- cortusaefolius $\boldsymbol{W}$.

- millefoliatus Vahl.

- muricatus $L$.

- pedatus Kit.

- Stevenii Bess.

- tuberculatus Dec.

Reseda alba $L$.

- crispata $L k$.

- Phyteuma L.

Rhagadiolus edulis Gaertn.

- Koelpinia $W$.

- stellatus $W$

Rhenm compactum $\mathbf{L}$.

- erispum.

- nutans.

- Rhaponticum $\boldsymbol{L}$.

- sibiricum.

- tataricum $L$

- tauricum.

- undulatum $L$.

Ribes aurenm Pursh.

Ricinus communis $L$.

Rivina laevis $\boldsymbol{L}$.

- purpurascens Schrad.

Resa ruhifolia $R$. $B r$.

Rudbeckia amplexifolia Jacq.

- laciniata $L$. triloba $L$.

Ruellia capensis,

Rumex alpinus $L$.

- aquaticus $\boldsymbol{L}$.

- cristatus Dec.

Rumex domestiens Hartm.

- maximus Schreb.

- nemorosus Schrad.

- Patientia $L$.

- pratensis Koch.

- pulcher $L$.

- purpureus Poir.

- salicifolius $\boldsymbol{W}$ einm.

- sanguineus $\boldsymbol{L}$.

- sylvestris $W$.

Ruta chalepensis $L$

Salsola Tragus $L$.

Salvia Aethiopis $\boldsymbol{L}$.

- austriaca $L$.

- campestris M. B.

- disermas $L$.

- glutinosa $\boldsymbol{L}$.

- grandiflora Etling.

- hirsuta Jacq.

- hispanica $L$.

- indica $L$.

- interrupta Schousb.

- Sclarea $L$

- truncata Donn.

- Verbenaca $L$.

- virgata Ait.

Sambucus canadensis $L$.

Samolns.Valerandi $\boldsymbol{L}$.

Sanvitalia procumbens $W$.

Saponaria Vaccaria $\boldsymbol{L}$.

Satureja euneifolia $T e n$.

- montana $L$.

Saracha viscosa Schrad.

Saxifraga Aizoon $L$.

- hirsuta $L$.

- intacta $W$

- pyramidalis Lap.

- rotundifolia $L$.

- serrata Ott

- spathulata Desf.

Scabiosa alpina $L$.

- atropurpurea $L$.

- australis Wulf.

- caucasica M. B.

- centauroides Lam.

- corniculata Kit.

- graminifolia $L$

- Hladnikiana Host.

- stellata $L$

- uralensis Murr.

Seandix brachycarpa Guss.

Schismus marginatus $P, B$. 
Seseli gummiferum $\mathbf{S}$.

- montanum $L$.

- rigidum Kit.

- tortuosum $L$.

Sesleria elongata Host.

Sida angustifolia Juss.

- Napaea Cav.

- triloba Cav.

- vesicaria Cav.

Sideritis montana $\boldsymbol{L}$.

Silene alpestris Jacq.

- aprica Turcz.

- Atocion Jacq.

- chlorantha Ehrh.

- compacta

- flavescens Kit.

- fruticosa $L$.

- gallica $L$.

- gracilis Dec.

- hirsuta Lag.

- imbricata Desf.

- inamoena Lindl.

- infracta Kit.

- linaccola Wib.

- livida $W$.

- mollissima $S m$.

- pendula $L$.

- quinquevulnera $L$.

- regia Sims. ${ }^{7}$ )

- saponariaefolia Schott.

- sericea All.

- viridiflora $L$.

Sinapis auriculata Dec. turgida Delil.

Sisymbrium austriacum Jacq.

- strictissimum $\boldsymbol{L}$.

Sisyrinchium Bermudiana $\boldsymbol{L}$.

- $\operatorname{laxum} L k$.

- striatum Cav.

Solanum campechiense $\boldsymbol{L}$.

- flavum Ait.

- marginatum $\boldsymbol{L}$.

- ovigerum Dun.
Solanum Pseudocapsicum $\boldsymbol{L}$.

- radicans $L$.

- sodomeum $L$.

- Zuccagnianum Dun.

Sonchus flexuosus Ledeb.

- leucophaeus $W$.

palustris $L$.

Sorghum cernuum $W$.

- saccharatum Pers.

- vulgare v. pyramidatum.

Spartina cynosuroides Roth.

Spartium junceum $L$.

Spilanthes oleracea $L$.

Stachys alpina $L$.

- lanata Jacq.

- polystachya Ten.

- purpurea Poir.

Stachytarpheta jamaicensis Vahl.

Statice latifolia $S m$.

- Limonium $L$.

- palmaris $S m$.

- sinuata $L$.

- tatarica $L$.

Stipa capillata $L$.

gigantea Lag.

Stylidinm adnatum $\boldsymbol{R} . \boldsymbol{B r}$.

- fruticosum $R . B r$.

Symphoria racemosa Pursh.

Syrenia Biebersteinii Andrz.

Tagetes erecta $L$.

- patula $L$.

- tanacetifolia H. Par.

Talinum purpurenm Fisch.

Tanacetum boreale.

Teedia lucida Rud.

Tellima grandiflora Lindl.

Tencrium flavum $\boldsymbol{L}$.

- hyrcanicum $\boldsymbol{L}$.

- massiliense $L$.

- Pseudo-Scorodonia Desf.

Thrincia hirta Roth.

Thymus canus Stev.

- Crantzianus H. Genev. grandiflorus.
Thymus neglectns $\boldsymbol{H}$. Genev. - Nepeta Scop.

Tiaridium indicnm Lehm.

Tolpis barbata Gaertn.

Tordylium àpulum Riv. maximum $L$. syriacum $\boldsymbol{L}$.

Tradescantia erecta Cav.

- Selloviana Pohl.

Tragopogon campestris Bess.

- floccosus Kit.

- porrifolius $L$.

Tribulus terrestris $L$.

Trifolium elegans Sav.

- incarnatum $\boldsymbol{L}$.

- libanoticum Ehrenb.

- ligusticum Balb.

- subterraneum $L$.

- vesiculosum Sant.

Trigonella arcuata Meyer.

- Calliceras Fisch.

- cancellata Fisch.

- Foenum graecum $\boldsymbol{L}$.

- tenuis Fisch.

uralensis Led.

Trinia vulgaris Dec.

Triticum compositum $L$

- cristatum Schreb.

- dasyanthon Ledeb.

- desertorum Fisch.

- fastuosum Lag.

- junceum $\boldsymbol{L}$.

- polonicum $L$. turgidum $\boldsymbol{L}$.

Triumfetta oblongata $L k$.

- triclada $L k$.

Urospermum Dalechampii Desf.

- picroides Desf.

Urtica cannabina $\boldsymbol{L}$.

- convexa Horn.

Valantia táurica $W$.

Valerianella coronata Dec.

- tridentata $L$.

- uncinata Dufr.
Verbascum anstrale Schrad.

- blattarioides Lam.

- condensatum Schrad.

- orientale $M$. $B$.

- rotundifolium $T$ en

Verbena bonariensis $\boldsymbol{L}$.

- bracteosa $M x$

- hastata $L$.

- lasiostachys $L k$.

- paniculata Lam.

- stricta Vent.

- urticaefolia $L$.

Vernonia anthelminthica $W$

Veronica biloba Vahl.

- brevifolia $M$. $B$.

- caucasica M. $B$. virginiea $L$.

Vesicaria cretica Poir.

- sinuata Poir.

- utriculata Lam.

Viburnum lantanoides $M x$.

Vicia atropurpurea Desf.

- biennis $\boldsymbol{L}$.

- bithynica $\boldsymbol{L}$.

- dasycarpa Ten.

- globosa Retz.

- lutea L.

- Nissoliana Gouan.

- tenuissima $\boldsymbol{H} . \boldsymbol{B e r}$.

Viola hederacea Labill

- persicifolia.

Xanthium orientale $\boldsymbol{L}$.

- spinosum $\boldsymbol{L}$.

Ximenesia encelioides Cav.

Zephyranthes candida Herb.

Zinnia elegans var. coccinea

- tenuiflora"Jacq violacea.

- verticillata $A n d r$

Ziziphora dasyantha $M$. $B$.

- serpyllacea.

Zoegea Leptanrea $\boldsymbol{L}$.

Zygophyllum Fabago $L$.

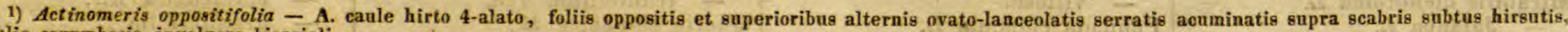
capitulis corymbosis, involucro biseriali.

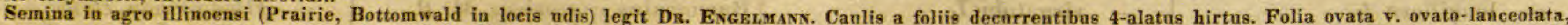

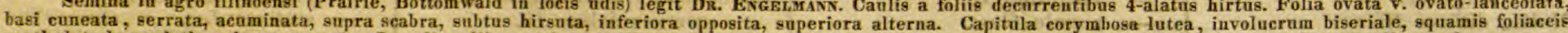

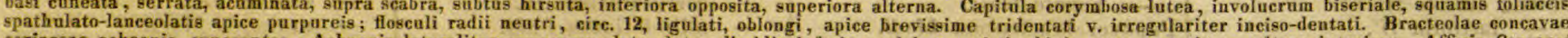

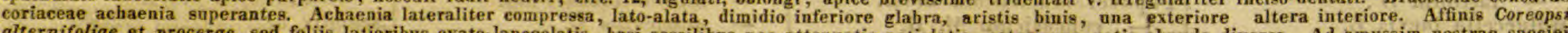

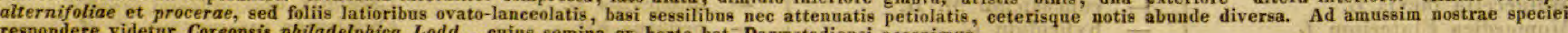
respondere videtur Coreopsis philadelphica Lodd., cujus semina ex horto bot. Darmstadiensi accepimus.

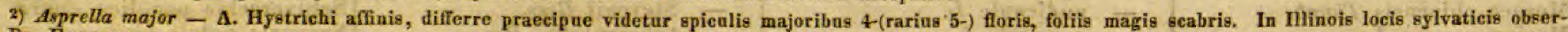
vavit Dr. ENGELMANN.

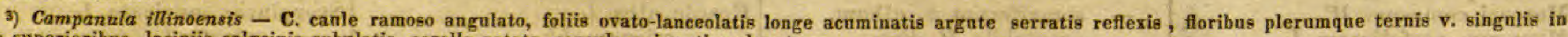
axillis superioribus, laciniis calycinis subulatis, corolla rotata, capsula prismatico-clavata.

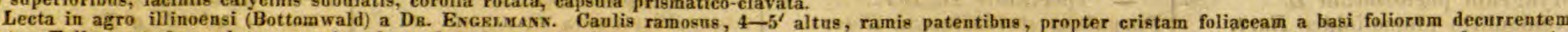

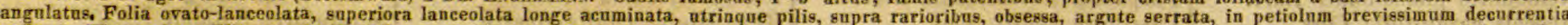

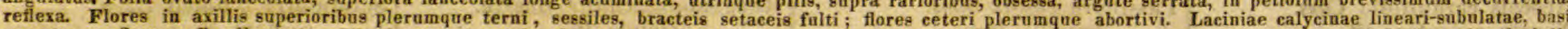

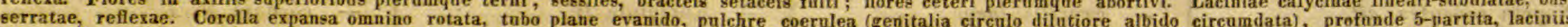

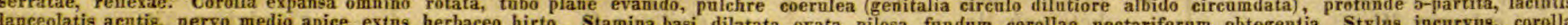
longior, stigma trifidum. Capsula prismatico-clavata, trilocularis, 5-6 $6^{\prime \prime \prime}$ longa, poris tribus gub apice dehiscens.

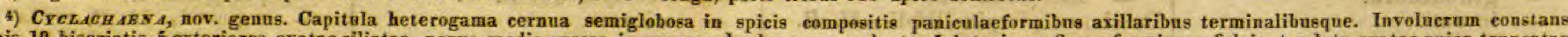

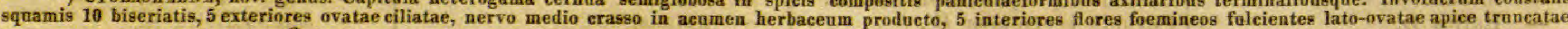

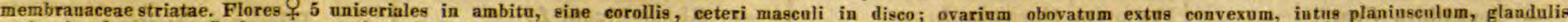

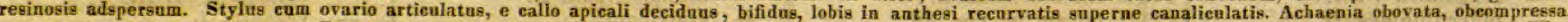

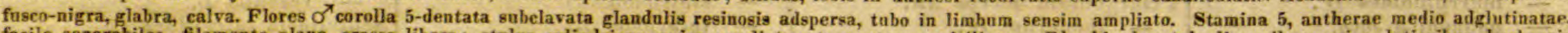

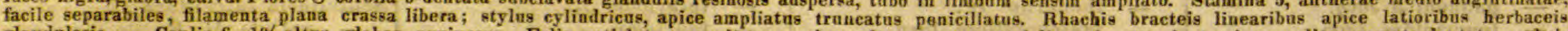

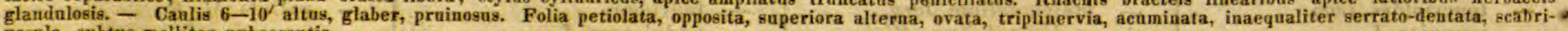
uscula, subtus molliter pabescentia.

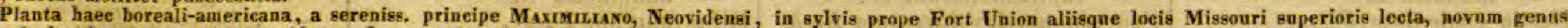
e tribu Ambrosiearum constituere videtar, Ivae proximum, sed praecipue floribug foemineis apetalis distinctum.

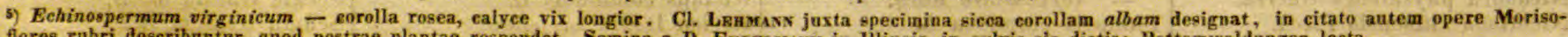
niano flores rubri describantur, guod nostrae plantae respondet. Semina a D. Evesumavi in IIlinois, in sylvis sie dictis: Bottomwaldungen lecta.

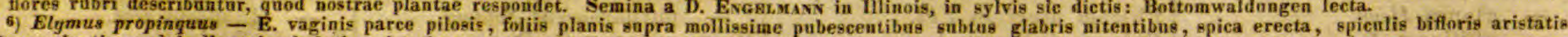
villosis geminatis, valvis lineari-subulatis aristatis nervosis spicnlis brevioribes.

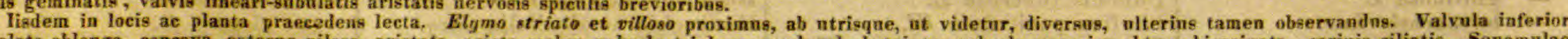

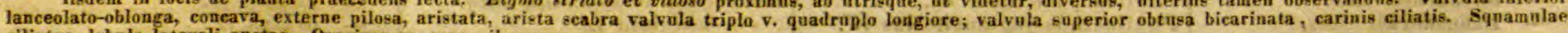
ciliatae, lobulo laterali anctse. Ovarium superne pilosum.

7) Semina ex Illinoig misit Dr. Evgermanv. 
Acacia armata $R . B r$.

- Farnesiana $W$.

- leucocephala Berter.

- lophantha $W$

Achillea biserrata $\dot{M}$. $B$.

- pubescens $L$.

- setacea $W$. Kit.

Achyranthes brachiata $\boldsymbol{L}$.

fruticosa $\mathrm{Lam}$.

Achyropappus sehkuhrioides $L k$.

Aconitum tauricum.

- volubile Pall. Vulparia Reichb.

Acroglochin chenopodioides Schrd.

Actaea brachypetala Dec.

- racemosa $L$.

Adenocarpus foliolosus Dec.

Adenophora communis Fisch,

Aegilops cylindrica Host.

- ovata $L$

- squarrosa $L$.

- triuncialis $L$.

Aethionema cappadocicum Spr.

saxatile $R$. $B r$

Agrimonia suaveolens Pursh.

A gropyrum seirpeumi Guss.

Agrostis elegans.

- laxa Schreb.

Airochloa villosa Link.

Aldeaea pinnata $\boldsymbol{R}$. et $\boldsymbol{P}$.

Ajuga Chamaepitys Sehreb.

Allionia nyctaginea Mchx.

Allium acutangulum $W$.

- albidum Fisch.

- baicalense $\boldsymbol{W}$.

- cernuum Roth.

- Cyrilli Ten.

$\rightarrow$ fragrans Vent.

- neapolitanum Cyrill.

- ramosum Jacq.

- senescens $L$.

- sibiricum $L$.

- striatum Jacq.

- tauricum.

- tenuiflorm Ten.

- Victorialis $L$.

Alopecurus lasiostachyus Link.

Alstroemeria Pelegrina $L$.

Althaen Froloviana Fisch.

- Sieberi $L k$

Alyssum saxatile $\boldsymbol{L}$.

Amarantus speciosus Ker. spinosus $L$.

Ambrosia trifida $L$

Amethystea coerulea $\boldsymbol{L}$.

Ammannia latifolia $\boldsymbol{L}$.

Ammi majus $L$.

- Visnaga Lam.

Armobium alatum $R$. $B r$.

Anacyclus Pyrethrum $L k$.

Anchusa asperrima Delil.

- hybrida Ten.

- paniculata Ait.

- rosea $M . B$.

- sempervirens $\boldsymbol{L}$.

Andropogon furcatus Mïhlenb,

Androsace septentrionalis $\boldsymbol{L}$.

Androsaemum officinale $\mathrm{All}$.

Andryala cheiranthifolia Herit.

- lanata $L$.

Anemone canadensis.

- Hudsoniana Richards.

- ochotensis Fisch.

Angelica atropurpurea.
Angelica decurrens Led.

Anisodus luridus Link.

Anoda hastata Cav.

Anthemis rigescens $W$.

- ruthenica $M . B$.

- secundiramea Biv.

Anthericum Liliago $L$.

Anthyllis tetraphylla $L$.

Antirrhinum elegans Pers. sempervirens Lap. siculum Uer.

Apargia saxatilis Ten.

Aquilegia glandulosa Fisch.

- sibirica Lam.

speciosa $H$. Marb.

Arabis collina $T e n$.

- crispata $W$.

- Jilacina Schrad.

- nivalis Spr.

- exyota Dec

- rosea Dec.

Arachis hypogaea $L$.

Arenaria calycina Poir.

- rigida $M . B$.

- rostrata Kit.

Argemone Barklayana Grah.

- mexieana $\beta$. albiflora.

Artemisia Sieversiana Ehrh.

- violacea Desf.

Arthropodium pendulum Spr.

Asclepias angustifolia $W$.

- curassavica $L$

- incarnata $L$

- nivea $L$.

Asperula montana Kit.

Asphodelus clavatus Roxb.

- creticus Lam.

- luteus $L$.

- tauricus M. B.

Asprella Hystrix $W$.

- major Fresen.

Astragalus alopecuroides $\boldsymbol{L}$.

- asper Jacq.

- baeticus $L$.

- brachyceras Led.

- carolinianus $\boldsymbol{L}$.

- Cicer L.

- cymbaeformis Brot.

- galegiformis $\boldsymbol{L}$.

- subulatus Pall.

- sulcatus $L$.

- virescens Ait.

Athamanta flexuosa Desf.

- Matthioli $\boldsymbol{W u l f}$.

Athanasia annua $\boldsymbol{L}$.

Atheropogon apludoides $\boldsymbol{W}$.

Atropa Belladonna $L$.

Aubrietia Columnae Guss. deltoidea Dec.

Avena brevis' Roth.

- chinensis.

- planiculmis Schrad. rigida $M . B$.

Baccharis ivaefolia $L$.

Balbisia elongata $W_{\text {: }}$

Barbarea praecox $R, B r$.

Barkhausia al pina $L k$.

- graveolens $R c h b$.

- rubra $L k$.

Basella rubra $L$.

Beta foliosa Ehrenb.

Betoniea ineana Ait.

- orientalis $L$.

Bidens bipinnata $\boldsymbol{L}$.
Bidens grandiflora Balb.

- Jeucantha $W$.

Biscutella apula $L$.

- erigerifolia Dec.

- eriocarpa Dec.

- lyrata $L$.

- maritima Ten.

Biserrula Pelecinus $L$

Blumenbachia insignis Schrad.

Bonjeania microphylla Reichb.

Brachypodium asperum $R$. et Sch.

- longifolium P. B.

Briza maxima $L$.

- virens $L$

Brizópyrum siculum $L k$.

Bulbine annua $W$.

Bunias Erncago $L$

Bunium peucedanoides $M$. B.

Buphthalmum grandiflorum $L$.

- salicifolium $L$.

spinosum $L$.

Bupleurum altaicum Led.

- falcatum $\boldsymbol{L}$.

- heterophyllum $L k$.

- multinerve Dec.

- nervosum Trev.

- sibiricum Trev.

Cacalia atriplicifolia $L$.

- sonchifolia $L$.

Cakile perennis $D e c$

- rugesa Dec.

Calamagrostis argentea $D e c$.

Calamintha rupestris Host.

Calandrinia compressa Schrad.

- discolor Sclerad. grandiflora Lindl.

Caldasia heterophylla $W$.

Calendula aegyptiaca Desf.

- Asterias Fisch, et M.

- pluvialis $L$.

- stellata Cav.

Callistachys Ianceolata Vent.

Camelina dentata Pers.

Campanula betonicaefolia $\boldsymbol{M}$. $\boldsymbol{B}$. divergens $W$.

- Erinus L.

- hybrida $L$.

- latifolia $I$.

- obliquifolia Ten.

- peirescifolia Fisch.

- peregrina $L$.

- pyramidalis $\boldsymbol{L}$.

- urticaefolia Schmidt.

Canna speciosa Rosc.

Capraria biflora $L$.

Capsicum bicolor Jacq.

- chinense $\boldsymbol{L}$.

- pendulum $W$.

Cardiospermum Halicacabum $\boldsymbol{L}$.

Carex depauperata Good.

- divulsa Good.

- juncea $H$.

- laevigata $S m$. nutans Host.

Carthamus tinctorius $L$.

Carum Bulbocastanum Koeh.

Cassinia spectabilis $R$. Br.

Catananche coerulea $L$.

Caucalis grandiflora $L$.

Celosia castrensis $L$.

- cristata $L$.

- margaritacea $\boldsymbol{L}$.

- virgata Jacq.

Celsia Arcturns $L$.

Celsia australis.

- lanata Jacq.

Cenchrus echinatus $L$.

Centaurea calocephala $W$.

- cicutaefolia Horn.

- Crocodilium L.

- Crupina $L$.

- depressa M. $\boldsymbol{B}$.

- eriophora $\boldsymbol{L}$.

- melitensis $\boldsymbol{L}$.

- moschata $L$.

- ragusina $L$.

- rupestris $L$.

- ruthenica Lam.

- sonchifolia $\boldsymbol{L}$.

- sulphurea $W$

- tatarica $L$

Centrospermum Chrysanthemum Spreng.

Cerastium davurieum Fiseh.

- grandiflorum Kit.

- Ledebourianum Dec.

Ceratocephalus falcatus Pers.

Ceratochloa pendula Schrad.

- unioloides Dec.

Cerinthe aspera Roth.

- major $L$.

Chaerophyllum trichospermum

Cheirinia crepidifolia $L k$.

Chelidonium laciniatum Mill.

Chelone campanulatia Cav.

- conferta $R, B$.

- diffusa Dougl.

- Digitalis Spr.

- glandulosa.

- hirsuta $L$.

- ovata Dougl.

Chenopodium ambrosioides $L$.

- Botrýs L.

- chilense Schrad.

- Quinoa $W$.

Chorizema ilicifolium Labill.

- rhombenm R. Br.

Chryseis tenuiflora H. Jen.

Cicer arietinum $\boldsymbol{L}$.

Cicuta maculata $L$.

Cinna expansa Link.

Cirsium laniflorum $M, B$. scleranthon $\boldsymbol{M} . \boldsymbol{B}$.

Cistus candidissimus Dun.

- undulatus.

- vaginatus Ait.

Clarkia elegans Dougl.

- pulchella Pursh.

Claytonia perfoliata Don.

Clematis angustifolia Jacq.

Cleome graveolens Rafin.

- spinosa $L$.

- violacea $L$.

Clintonia elegans Dougl.

Cneorum tricoccon $L$

Cnicus syriacus $\mathrm{W}$.

Cnidium apioides $S p r$.

Coix exaltata Jacq

Collomia coccinea Lehm.

- grandiflora Dougl.

Colpodium Steveni Trin.

Commelina angustifolia M.x.

- clandestina Mart. 
Corchorus aestuans $L$

- trilocularis $L$

Coreopsis Atkinsoniana Dougl. lanceolata $L$.

Coriaria myrtifolia $L$.

Corispermum canescens Kit.

Cornucopiae cucullatum $L$.

Coronilla coronata $L$.

- glauca $\boldsymbol{L}$

- Securidaca $\boldsymbol{L}$

Corydalis aurea $\boldsymbol{W}$.

- nobilis Pers.

- sempervirens Pers.

Crambe grandiflora Stev.

- maritima $L$

Crepis heterosperma Schrad.

Crotalaria sagittalis $L$.

Crucianella angustifolia $\boldsymbol{L}$.

Cucubalus bacciferus $\boldsymbol{L}$.

Cucumis prophetarum $\boldsymbol{L}$.

Cncurbita lagenaria $\boldsymbol{L}$.

Cuphea procumbens Cav.

- viscosissima Jacq.

Cyclachaena xanthifolia Fresen.

Cynanchum fuscatum Link.

- nigrum Pers.

Cynoglossum apenninnm $L$.

- bicolor $\boldsymbol{W}$.

- sylvaticum $\mathbf{S m}$.

Cyperus alternifolius $L$.

- spectabilis Schreb.

Cytisus capitatus Jacq.

- hirsutus $L$.

- prostratus.

Dactylaena micrantha Sehrad.

Datisca cannabina $\boldsymbol{L}$.

Datura Tatula $L$.

Dancus hispidus Desf.

- maritimus $\boldsymbol{L}$

- muricatus $L$.

Delphinium grandiflorum $\boldsymbol{L}$.

- montanum Dec.

- Requienii Dec.

- Staphisagria $\boldsymbol{L}$.

Desmanthus virgatus $W$.

Dianthus collinus Kit.

- dintinus Kit.

Dictamnus albus $\boldsymbol{L}$.

Digitalis australis Ten.

- Buxbaumii Bess.

- ferruginea $L$.

- grandiflora Lam.

- laevigata Kit.

- lanata Ehrh.

- lutea $\boldsymbol{L}$.

Digitaria aegyptiaca $\boldsymbol{W}$.

Dinebra curtipendula Dec.

Diplachne fascicularis $P . B$.

Dipsacus laciniatus.

Dolichos brasiliensis.

- Catiang $\boldsymbol{L}$.

- Lablab L.

- purpureus $\boldsymbol{L}$.

Derycnimm herbaceum $\mathrm{Vill}$.

Draba aizoides $L$.

- aurea $V a h l$.

- contorta Ehrh

Dracocephalum canariense $\boldsymbol{L}$.

- canescens $\boldsymbol{L}$.

- Moldaviea $L$.

- peltatum $\boldsymbol{L}$.

- sibiricum $L$.

Eccremocarpus scaber $\boldsymbol{R}$. et $\boldsymbol{P}$.

Echinops Ritro $\boldsymbol{L}$.

- sphaerocephalus $L$.

Echium creticum $L$.

- grandiflerum Desf.

- italicum $\boldsymbol{L}$.

- salmanticum Lag.

Eclipta erecta $\boldsymbol{I}$.
Eclipta Iatifolia $\boldsymbol{L}$.

- prostrata $L$.

Eleusine coracana Pers.

- indica Lam.

- rigida $S_{p}$.

- Tocussa Fres.

Elichrysum bracteatum Vent. fulgidum $W$.

Elsholtzia cristata $W$.

Elymus arenarius $\boldsymbol{L}$.

- canadensis $L$.

- carolinianus.

- giganteus Vahl.

- propinquus Fresen.

Emex spinosus Camp.

Entelea arborescens $\boldsymbol{R} . \boldsymbol{B r}$.

Epilebium angustissimum Ait.

Eragrostis abyssinica $L k$.

- namaquensis Nees ab Es.

Eranthis hyemalis Salisb.

Erigeron contortus Desf.

- elongatus Led.

- glabellus Nutt.

- podolieus Bess.

- Villarsii Bell.

Erinus alpinns $\boldsymbol{L}$.

Erodium gruinum Ait.

- moschatum Ait.

- pulverulentum $W$

Ervum agrigentinum Guss.

- Ervilia $\boldsymbol{L}$.

Eryngium giganteum $M$. $B$.

- planum $\boldsymbol{L}$.

Erysimum altaicum Meyer.

- hieracifolium.

- odoratum Ehrh.

Eschscholzia ealifornica Cham.

Ethulia conyzoides $L$.

Enpatorium ageratoides $\boldsymbol{L}$.

- album $\boldsymbol{L}$.

Euphorbia cyathophora Jacq.

- Humbeldtii $W$

- Lagascae Spr.

- Lathyris $\boldsymbol{L}$.

- prunifolia Jacq.

Exacum viscosum $\boldsymbol{S} m$.

Farsetia clypeata $\boldsymbol{R}, \mathrm{Br}$.

- eriocarpa Dec.

Fedia Cornucopiae $\mathrm{Vahl}$.

Ferula campestris Bess.

- Ferulago $\boldsymbol{L}$.

Flaveria repanda $\mathrm{Lag}$.

Forskolea angustifolia Retz.

- tenacissima $\boldsymbol{L}$.

- viridis Ehrenb.

Francoa sonchifolia $\mathbf{S p r}$.

Fuchsia arborescens Sess.

- coccinea $L$.

Fumaria capreolata $\boldsymbol{L}$

Galardia bicolor Lam.

Galinsogea trilobata Cav.

Galium lucidum.

- spurium.

- verrucostum $S_{m}$.

Ganra biennis $\boldsymbol{L}$.

- tripetala Cav.

Genista canariensis $\boldsymbol{L}$.

- hispaniea $\boldsymbol{L}$.

- ovata Kit.

- virgata $W$.

Gentiana cruciata $\boldsymbol{L}$.

Geranium carolinianum $\boldsymbol{L}$.

- sanguineum $\boldsymbol{L}$.

Geropogen glaber $\boldsymbol{L}$.

Geum album Gmel.

- atlanticum Desf.

- coccineum Sm.

- heterophyllum.

japonieum Thunb.

- ranunculoides Ser.
Gilia achilleaefolia Benth. - capitata Hook.

- inconspicua $\mathrm{Hook}$.

- laciniata $\boldsymbol{R}$. et $\boldsymbol{P}$

- tricolor Benth.

Gladiolus psittacinus. Hook.

Glaucium cornicnlatum Pers.

- fulvum Sin.

- luteum Scop.

- violaceum Sm.

Glycine Comptoniana Andr.

Glycyrrhiza echinata $L$.

- foetida Desf.

- glabra $L$

glandulifera $\mathrm{Kit}$.

Gnaphalium foetidum $\boldsymbol{L}$.

Gomphrena deeumbens Jaeq. globosa $\boldsymbol{L}$.

Goodenia grandiflora Sims.

Grahamia aromatica Hook.

Grewia occidentalis $\boldsymbol{L}$.

Grindelia incisa $S p r$.

Gynandropsis murieata Schrad.

Gypsophila acutifolia Fisch.

- altissima $L$.

- collina Stev.

- elegans M. B.

- glauca Stev.

- repens $L$.

- Steveni Fisch.

- stricta Bunge.

Hablizia tamnoides M. $B$.

Haloragis Cercodia Ait.

Hasselquistia cerdata $L$.

Hedypnois pendula $W$.

Hedysarum al pinum $L$.

- canadense $\boldsymbol{L}$.

- coronarinm $L$

- flexuosum $\boldsymbol{L}$. sibiricum Lam.

Heimia salicifolia $L$.

Helenium autumnale $\boldsymbol{L}$.

- mexicanum Humb.

- quadridentatum Lab.

Helianthemum aegyptiacum Mill. - racemosum Pers.

Helianthus lenticularis Lindl.

Heliophila amplexicaulis $\boldsymbol{L}$.

Hemimeris urticaefolia $W$.

Heracleum angustifolium $L$.

- asperum $\boldsymbol{M}$. $\boldsymbol{B}$.

- dissectum Led.

- Panaces L.

- speciosum.

- villosum Fisch.

Hermannia denudata $L$.

Heterospermum pinnatum Cav.

Heterotheca inuloides Cass.

Hibiscus radiatus Cav.

- syriacus $\boldsymbol{L}$.

- Trionum $L$.

- $\quad$ B. ternatus Cav.

- " var. grandiflora.

Hieracium amplexicanle $\boldsymbol{L}$.

- andryaloides Vill.

- angustifolium $\boldsymbol{H o p p .}$

- ceratophyllum Led.

- corymbosum Led.

- denudatum Lapeyr.

- glaueum Alt.

- Halleri Vill.

- prenanthoides Vill.

- sabandum L.

Hiorthia aurea Less.

Hippocrepis unisiliquosa $L$.

Holosehoenus atrovirens $L \boldsymbol{k}$,

Hordenm bulbosum $\boldsymbol{L}$.

- coeleste Vib.

- maritimum $\boldsymbol{H}^{\text {ither. }}$

- nigrum $W$.

Hordeum nudum.

- strictum Desf.

- Zeocriton $\boldsymbol{L}$.

Horminuin caulescens Orteg.

Humea elegans $S m$.

Hydrocotyle sibthorpioides Lam.

Hyoscyamus aureus $L$.

- canariensis $K e r$.

physaloides $\boldsymbol{L}$.

Hyoseris arenaria Schousb.

- scabra $L$.

Hypecoum procumbens $L$.

Hypericum elegans $\boldsymbol{W}$.

veronense.

Hypochoeris arachnites Biv.

Hyssopns angustifolius $M$. $B$.

- Foeniculnm Spr.

- Lophanthis $\boldsymbol{L}$.

- nepetoides $L$.

- officinalis $L$.

- orientalis $W$.

Impatiens parviflora Dec.

Indigofera australis $\boldsymbol{W}$.

Inula bifrons $L$.

- suaveolens Jacq.

Ipomoea coccinea $L$.

Ipomopsis elegans $M \boldsymbol{x}$.

Iris halophila.

- Güldenstädtiana Lepech.

- maritima Mill.

- mauritiana.

- ochrolenca M. B.

- plicata Lam.

- spuria.

- - B. minor stenogyna Dec.

Isatis dasycarpa Led.

Isopyrum fumarioides $\boldsymbol{L}$.

Isotoma axillaris Lindl.

Juncus tenuis $W$.

Justicia sarmentosa.

Ixia Bulbocodium.

Kalbfussia Milleri Schultz.

Kaulfussia amelloides Nees.

Kennedia apetala Lodd.

- prostrata $R$. $B r$.

- rubicunda $V$ ent.

Kitaibelia vitifolia $\boldsymbol{W}$.

Kleinia suffruticosa $\boldsymbol{W}$.

Knantia orientalis $\boldsymbol{L}$.

Kochia hyssopifolia Roth.

Koeleria aegyptiaca. 
Leonurus nepalensis.

Lepidium suffruticosum $L$.

Lepidonema chilensis Fisch. et M.

Lessertia perennans Dec.

Levisticum officinale Koch.

Libanotis Buchtormensis Dec.

- vulgaris Dec.

Ligusticum alatum Spr. Seguieri Koch.

Linaria bipartita $W$.

- genistaefolia Mill.

- litoralis Bernh.

- purpurea Mill.

- pyrenaica Dec.

- triphylla $\boldsymbol{W}$.

Lindenbergia urticaefolia Lehm.

Linum alpinum $L$.

- narbonense $L$.

- suffruticosum $L$.

Lobelia inflata $L$

- triquetra $L$.

Lolium speciosum Stev.

Lopezia coronata $A n d r$.

Lophospermum scandens Don.

Lotus edulis $\boldsymbol{L}$.

- Jacobaeus $L$.

- ornithopodioides $L$.

- siliquosus $\boldsymbol{L}$.

Lupinus albus $L$.

- angustifolius $\boldsymbol{L}$.

- bicolor.

- Cruikshankii Bot. Mag.

- hirsutus $L$.

- linifolius Roth.

- lituratus Reichb.

- Inteus $L$.

- pilosus $L$.

- polyphyllus Dougl.

- pulchellus Dougl.

- Termis Forsk.

Luzula maxima Dec.

Lychnis fulgens Fisch.

Lycopus exaltatus $L$ irginicus $L$.

Lysimachia ciliata $L$.

- dubia Ait.

- Ephemerum $\boldsymbol{L}$.

- verticillata Pall.

Lythrum verticillatum $L$.

- virgatum $\boldsymbol{L}$.

Madia elegans Don.

- quinqueradiata Fisch, et $M$.

Malcolmia maritima $R$. $B r$.

Malope grandiflora Hort.

- malacoides $L$.

Malva commutata Reichb.

- crispa.

- limensis $L$

- parviflora $L$.

Mandragora officinalis Mill.

Martynia proboscidea Glox.

Maurandia Barklayana Lindl.

- semperflorens Jacq.

Meconopsis cambrica $\mathrm{Vig}$

Medicage apiculata $W$.

- glutinosa M. B.

- intertexta Desr.

- marginata $\boldsymbol{W}$.

- microdon Ehrenb.

- pentacycla Dec.

- praecox Dec.

- tribuloides Desr.

- turbinata $W$

Megastachya rigida Beauv.

Melampodinm longifolium Brouss.

Melananthera Linnaei Kunth.

Melica altissima $\boldsymbol{L}$.

Melilotus caerulea Desv. " var. connata. indica Desv.
Melilotus rotundifolia Ten. - rugulosa $W$. ruthenica $M$. . .

Melissa alba Kit.

Menispermum canadense $\boldsymbol{L}$.

Mesembrianthemum cordifolium $L$. - glaciale Haw.

- pugioniforme $t$.

Meum athamanticum Jacq.

Milium frutescens Sieb.

Mimosa Farnesiana.

- pudica $L$.

Mimulus floribundus Lindl.

- glutinosus $\boldsymbol{W e n d l}$.

- guttatus Dec.

- Inteus $L$.

- moschatus Dougl.

- roseus.

Mirabilis dichotoma $L$.

- Jalappa $\boldsymbol{L}$.

longiflora $L$.

Mollia diffusa $\boldsymbol{W}$.

- latifolia $\boldsymbol{W}$

Molopospermum peloponnesiacum

Moluccella laevis $\boldsymbol{L}$.

Koch.

Momordica Elaterium $\boldsymbol{L}$

Moraea chinensis Thunb.

Morina persica $L$.

Mühlenbergia diffusa Schreb.

Muscari comosum $\boldsymbol{W}$.

Myarrum perfoliatum $\boldsymbol{L}$.

Nepeta coerulea Ait.

- colorata $W$.

- grandiflora.

- incana.

- italica $L$.

- longiflora Vent.

- marrubioides $W$.

- pannonica Jacq.

- teucrioides Lam.

ucranica $L$

Neslia paniculata Desv.

Nicandra physalodes Gaertn.

Nicotiana alata $L k$, et 0 tto.

- chinensis Fisch.

- fruticosa $L$

- glauca Hook.

- glutinosa $\boldsymbol{L}$.

Langsdorffii Nees.

- maerophylla $S p r$.

- noctiflora Hook.

vineaeflora Lag.

Nigella hispanica $\boldsymbol{L}$.

- orientalis $L$.

sativa $\boldsymbol{L}$.

Nolana grandiflora Horn. prostrata $L$

Nonea pulla Dec.

Oenothera chilensis cruciata Nutt.

- Fraseri Pursh.

- fruticosa $L$.

glanea $M x$

Lindleyana Sims.

muricata $L$.

odorata Jacq.

- purpurea Curt.

Romanzovii Led.

rosea Ait.

- Simsiana Ser.

- spectabilis Horn.

- tetraptera Cav.

- triloba Nutt.

- undulata.

Omphalodes linifolia Moench.

Onobroma creticum $S p r$.

- lanatum Spr.

Onobrychis Caput galli Lam.

- conferta spr.
Onobrychis Crista galli Lam.

Ononis alopecuroides $L$.

- fruticosa $L$.

- hircina.

- Natrix $L$

Onopordon illyricum $L$.

- macracanthum Schousb.

- viscosum.

Ornithogalum abyssinicum Fres.

- collinum Guss.

- narbonense $L$.

Orobus atropurpureus Desf.

- niger $L$.

- vernus $L$.

Paeonia humilis Retz.

- peregrina Mill.

- tenuifolia $L$.

- villosa.

Panicum colonum $L$.

- plicatum Lam.

- virgatum $L$.

Papaver bracteatum Lindl.

- hybridum $\boldsymbol{L}$.

- intermedium Alph. Dec.

- orientale $\boldsymbol{L}$.

- somniferum $\beta$. album.

- spectabile H. Ber.

- tauricum.

Paspalum stoloniferum Bosc.

Passiflora edulis Sims.

- gracilis Jacq.

- heterophylla.

- Raddiana Dec.

Pastinaca pimpinellifolia M. B.

Pavonia praemersa $W$

Peganum Harmala $L$.

Pelargonium inodorum $W$. malvaefolium Jacq.

Peucedanum involucratum Koch. - verticillare Koch.

Phacelia tanacetifolia.

Phaseolus Caracalla $L$.

Phleum asperum Vill.

Phlomis salviaefolia.

- tuberosa $L$.

Phyllanthus juglandifolins $W$.

Phyllis Nobla $L$.

Physalis flexnosa $L$.

Phyteuma Scheuchzeri $A l l$.

- strictum Ker.

- virgatum $W$.

Phytolacea decandra $L$.

- icosandra $L$.

Picris strigosa M. B.

Pimpinella aromatica $M$. $B$. peregrina $L$.

Pisum sativum L. $\%$ umbellatum.

Pittosporum undulatum $A n d r$.

Plumbago micrantha Desf.

Pocockia cretica Ser.

Podalyria australis $W$.

Polemonium coerulenm $W$.

- mexicanum Cerv.

Polygonum cymosum Trev.

- cmarginatum Roth.

- undulatum Murr.

Pomaderris elliptica $\mathbf{L a b}$.

- phylicaefolia $L k$.

Potentilla arguta Pursh.

- calabra Ten.

- canescens.

- collina Wib.

- formosa Don.

- geoides M. B.

- gracilis Dougl.

- Güntheri.

- recta $L$

- rupestris $L$.

- Thomasii Ten.

Poterium garganicum Ten.

Poterium guestphalicum Bönn. - spinosum $\boldsymbol{L}$.

Prismatocarpos falcatus Ten.

Prunella alba Pall.

- grandiflora $\boldsymbol{L}$.

- hyssopifolia $\boldsymbol{L}$.

- laciniata.

- pensylvanica $I T$.

Psoralea bituminosa $L$.

- bracteata $L$.

- capitata Thunb.

- hirta $L$

- pinnata $L$

- polystachya Poir.

- verrucosa $W$.

Pycnanthemum virginicum Pers.

Pyrethrum anethifolium $W$.

- corymbosum $W$

- macrophyllum $W$.

- millefoliatum $W$.

- roseum $M . B$.

- tenuifolium Ten.

Ranunenlus cancasicus $M$. $B$.

- illyricus $L$.

- muricatus $L$

- pedatus Kit.

- Stevenii Bess.

- tuberculatus Dec.

Reseda alba $L$.

- crispata $L k$.

- Phyteuma L.

Rhagadiolus edulis Gaert $n$.

- Koelpinia $W$.

- stellatus $W$.

Rheum compactum L.

- crispum.

- Rhaponticum $L$.

- sibiricum.

- tataricum $L$

- tauricum.

undulatum $L$.

Ribes aureum Pursh.

Ricinus communis $L$.

Rivina laevis $L$.

- purpurascens Schrad.

Rosa rubifolia $R$. $B r$.

Rudbeckia amplexifolia $\mathrm{Jacq}$.

- laciniata $L$

- triloba $L$.

Ruellia capensis.

Rumex alpinus $L$.

- cristatus Dec. 
Samolus Valerandi $\boldsymbol{L}$.

Sanvitalia procumbens $W$.

Saponaria Vacearia $L$.

Saracha viscosa Schrad.

Satureja montana $L$.

Saxifraga $\Lambda$ izoon $L$.

- hirsuta $L$.

- intacta $\boldsymbol{W}$.

- pyramidalis Lap.

- rotundifolia $L$.

Seabiosa alpina $L$.

- atropurpurea $L$.

- cancasica $M . B$.

- centauroides Lam.

- corniculata Kit.

- graminifolia $L$.

- stellata $L$

- uralensis Murr.

Scandix brachycarpa Guss.

Schismus marginatus $P . B$.

Schivereckia podolica Andrz.

Scoparia dulcis $\boldsymbol{L}$.

Scorpiurus muricata $\boldsymbol{L}$.

- sulcata $L$.

- vermiculata $L$.

Scrofularia altaica Murr.

- Balbisii Horn.

- cordata Pers.

- divaricata Led?

- mellifera Ait.

- peregrina $L$.

- Scorodonia $L$.

- variegata $M . B$.

- vernalis $\boldsymbol{L}$.

Seutellaria lateriflora $\boldsymbol{L}$.

- pallida M. $B$

- peregrina $L$.

- rubicunda Horn.

Secale fragile $M$. $B$.

Sedum lividum $\boldsymbol{W}$.

- Rhodiola Dee.

Senecio Doria $L$.

- elegans $L$.

- ovatus $W$

- venustus $A$ it.

Seriola aethnensis $L$

Serratula alata $\boldsymbol{W}$.

- cynaroides Dec. radiata $M$. $B$.

Seseli glaucum $L$.

- gummiferum $\boldsymbol{S} \boldsymbol{m}$.

- rigidnm Kit.

- tortuosum $L$

Sesleria elongata Host.

Sida Abutilon $L$.

- Napaea Cav.

Sida triloba Cav.

- vesicaria Cav.

Sideritis montana $\boldsymbol{L}$.

Siegesbeckia flosculosa Herit.

- orientalis $L$.

Silene alpestris Jacq.

- aprica Turcz.

- ehlorantha Ehrh.

- compacta Horn.

- cretica $L$.

- flavescens $K i t$.

- fruticosa $L$.

- gallica $L$

- hirsuta Lag.

- imbricata Desf.

- inamoena Lindl.

- infracta $K i t$.

- linaccola $W_{i b}$.

- livida $W$.

- mollissima $\boldsymbol{S}$.

- pendula $L$.

- quinquevulnera $L$.

- regia Sims.

- saponariaefolia Schott.

- sericea $A l l$.

- viridiflora $L$.

Sinapis aurieulata Dec. turgida Delil.

Sisymbrium austriacum Jacq. - strictissimum $\boldsymbol{L}$.

Sisyrinchinm Bermudiana $L$.

- striatum Cav.

Solanum campechiense $\boldsymbol{L}$.

- flavum Ait.

- guineense.

- marginatum $\boldsymbol{L}$.

- evigerum Dun.

- Prendocapsicum $L$.

- sodomeum $\boldsymbol{L}$.

Sonchns flexuosus Ledeb.

- lencophaeus $W$.

- palustris $L$.

Sorghum saceharatum Pers.

Spartina cynosuroides Roth.

Spartium junceum $L$.

Stachys alpina $L$

- cretica $L$.

- lanaia Jacq.

- polystachya Ten.

Statice latifolia $\mathrm{S} m$.

- elata Fisch.

- palmaris $\$ \mathrm{~m}$.

- scoparia Pall.

- sinuata $L$.

- tatarica $L$

Stenactis speciosa Lindl.
Stipa capillata $L$.

- gigantea Lag.

Streptinm asperum Roxb.

Sutherlandia frutescens $\boldsymbol{R}, \boldsymbol{B}$.

Stylidium adnatum $\boldsymbol{R}$. $\boldsymbol{B r}$.

Symphoria racemosa Pursh.

Syrenia Biebersteinii Andrz.

Tagetes erecta $L$.

- patula $L$.

Talinum purpureum Fisch.

Tanacetum boreale.

Teedia lncida Rud.

Telekia cordifolia Kit.

Tellima grandiflora $L i n d l$.

Teucrium flavum $L$.

- hyrcanicum $L$.

- massiliense $\boldsymbol{L}$.

- Psendo-Scorodonia Desf.

Thalictrum aquilegifolium $\dot{L}$.

Thrincia hirta Roth.

Thymus canus Stev.

- grandiflorus.

- Nepeta Scop.

Tiaridium indicum Lehm.

Tolpis barbata Gaertn.

Tordylium apulum Riv.

- maximnim $\boldsymbol{L}$. syriacum $L$.

Trachynia distachya Link.

Tradescantia erecta Cav.

- Selloviana Pohl.

Tragopogon eriospermus $T \mathrm{en}$.

- floccosus Kit.

- porrifolius $\boldsymbol{L}$.

Tribulus terrestris $L$

Trichonema ramiflora $T e n$.

Trifolium bracteatum Schousb.

- elegans Sav.

- incarnatum $L$.

- libanoticum Ehrenb.

- ligusticum Balb.

- subterraneum $\boldsymbol{L}$.

- vesiculosum Sant.

Trigonella arcuata Meyer.

- Calliceras Fisch.

- cancellata Fisch.

- Foenum graecum $L$.

- tennis Fisch.

- uralensis Led.

Trinia rulgaris Dec.

Triticum dasyanthon Ledeb.

Triumfetta oblongata $L k$.

Trollius altissimus $\boldsymbol{W}$ ender.

- cancasicus Stev.

- medius Wender.

Urachne paradoxa $L k$.
Urospermum Dalechampii Desf. - pieroides Desf.

Urtica cannabina $\boldsymbol{L}$.

- convexa Horn.

- pilulifera $\boldsymbol{L}$.

Valantia taurica $\boldsymbol{W}$.

Valerianella alliariaefolia $\mathrm{Vahl}$.

- coronata Dec.

- hamata Dec.

- uncinata Dufr.

Verbascum blattarioides Lam.

- condensatum Schrad.

- orientale $\boldsymbol{M}$. $\boldsymbol{B}$.

Verbena Aubletia Jacq.

- bonariensis $\boldsymbol{L}$.

- bracteosa $M x$.

- hastata $\boldsymbol{L}$

- lasiostachys $L k$.

- paniculata Lam.

- stricta Vent.

- urticaefolia $\boldsymbol{L}$.

- venosa.

Vernonia anthelminthica $\boldsymbol{W}$.

Veronica biloba $\mathrm{Vahl}$.

- brevifolia $\boldsymbol{M}$. $\boldsymbol{B}$.

- caucasica $M . B$.

- prostrata $\boldsymbol{L}$.

- virginica $L$.

Vesicaria cretica Poir.

- sinuata Poir.

Viburnum lantanoides $M x$.

Vicia articulata $\boldsymbol{L}$.

- biennis $L$

- bithynica $L$.

- dasycarpa Ten.

- globosa Retz.

- lutea $\boldsymbol{L}$.

- Nissoliana Gouan.

Viola hederacea Labill.

- persicifolia.

Wahlenbergia grandiflora

Schrad.

Xanthium orientale $L$.

- spinosum $L$.

Zacyntha verrucosa Gärtn.

Zephyranthes candida Herb.

Zinnia elegans var. coccinea.

- multiflora" $L$. violacea.

- tenuiflora Jacq.

- verticillata $A n d r$.

Ziziphora dasyantha $M$. $B$.

- serpyllacea.

Zoegea Leptaurea $\boldsymbol{L}$.

\section{G. Frestarte.}




\section{Semina in horto botanico Francofurtensi anno 1838 collecta.}

Acacia armata $R$. Br.

- Farnesiana $W$

- lophantha $W$.

- Iophantha var. coarctata.

Achillea Ageratum $\boldsymbol{L}$.

- alpina $L$.

- biserrata M. $B$.

- magna $L$.

- ochroleuca.

- odorata $L$

- pubescens $L$.

- setacea $W$. Kit.

- sylvatica.

tanacetifolia All.

Achyranthes brachiata $L$.

- fruticosa Lam.

- porrigens Jacq.

Achyropappus schkuhrioides $L k$. Acroglochin chenopodioides Schrd.

Actaea brachypetala Dec.

- racemosa $L$.

Actinomeris oppositifolia Fresen.

Adenocarpus foliolosus Dec. intermedius.

Adenophora communis Fisch,

Adlumia cirrhosa Rafin.

Aegilops cylindrica Host.

- ovata $L$.

- squarrosa $L$.

Aethionema cappadocicum Spr. saxatile $R . B r$

Agrimonia odorata.

- pilosa.

Agropyrum scirpeum Guss.

Agrostis elegans.

- laxa Schreb. mexicana $L$.

Airochloa villosa Link.

Ajuga Chamaepitys Schreb.

Aldeaca pinnata $R$. et $P$.

Allionia nyctaginea Mchx.

Allium acutangulum $W$

- albidum Fisch.

- atropurpureum Kit.

- azureum Led.

- baicalense $W$

- fragrans Vent.

- ramosum Jacq.

- sibiricum $L$

- striatum Jacq.

- tenniflorum Ten.

- Victorialis $L$.

Alstroemeria Pelegrina $\boldsymbol{L}$.

Althaea Froloviana Fisch.

- Sieberi $L k$.

Alyssum saxatile $\boldsymbol{L}$.

Amarantus speciosus $\mathrm{Ker}$.

- spinosus $L$

Ambrosia trifida $L$.

Amethystea coerulea $L$.

Ammannia latifolia $L$.

Ammi majus $L$.

- Visnagra Lam.

Ammobium alatum $R . B r$.

Amsonia latifolia $M x$.

Anacyelus bicolor.

- Pyrethrum Lk.

Anchusa Barrelieri Gmel.

- hybrida Ten.

- ochroleuca M. B.

- paniculata Ait.

- sempervirens $L$.

Andropogon furcatus Mühlenb.

Androsace maxima $L$.
Androsace septentrionalis $\boldsymbol{L}$. Androsaemum officinale $\mathrm{All}$. Andryala cheiranthifolia Herit. lanata $L$.

Anemone Hudsoniana Richards. - ochotensis Fisch.

Angelica decurrens Led.

Anisodus luridus Link.

Anoda hastata Cav.

Anomatheca cruenta Lindl.

Anthemis nobilis $\boldsymbol{L}$.

- rigescens $W$.

- ruthenica $M . B$.

- secundiramea Biv.

Anthericum Liliago $L$.

- Liliastrum $L$.

Anthyllis tetraphylla $L$

Antirrhinum elegans Pers. sempervirens Lap. siculum Uer.

Apargia saxatilis Ten.

Aquilegia canadensis $L$.

- glandulosa Fisch.

- sibirica Lam. speciosa $H$. Marb.

Arabis alpina $L$

- bellidifolia $L$.

- collina Ten.

- crispata $W$.

- lilacina Schrad.

- oxyota Dec.

- rosea Dec.

- Turrita $L$.

Arachis hypogaea $L$.

Arenaria calycina Poir.

- rigida $M . B$.

- rostrata $K$ Kit.

Argemone Barklayana Grah. - mexicana $\beta$. albiflora.

Artemisia Sieversiana $\mathrm{E} h \mathrm{r} / \mathrm{h}$.

- violacea Desf.

Arthropodium minus $R$. $B r$.

- pendulum Spr.

Asclepias angustifolia $\boldsymbol{W}$.

- curassavica $L$.

- incarnata $L$.

- nivea $L$.

Asperula montana Kit.

Asphodelus clavatus Roxb.

- creticus Lam.

- Inteus $L$

- tauricus $M . B$ tenuior $M . B$.

Asprella Hystrix $W$. major Fresen.

Astragalus alopecuroides $L$

- asper Jacq.

- baeticus $L$.

- brachyceras Led.

- carolinianus $L$.

- Cicer $L$.

- cymbaeformis Brot.

- galegiformis $L$.

- olopterus Fisch.

- subulatus Pall.

- suleatus $L$.

- uliginosus $\boldsymbol{L}$

- utriger Pall.

- virescens Ait.

Astrantia major $L$.

Athamanta flexnosa Desf.

- Matthioli $\boldsymbol{H}_{\text {ulf. }}$

thanasia annua $L$.

Atheropogen apludoides $W$ :
Aubrietia deltoidea Dec. Aulacospermum cuneatum Led.

Baccharis ivaefolia $\boldsymbol{L}$.

Balbisia elongata $W$.

Balsamita suaveolens Pers.

Baptisia minor.

Barbarea praecox $\boldsymbol{R}$. $B r$.

Basella rubra $L$.

Beta foliosa Ehrenb.

Betonica incana Ait.

- orientalis $L$.

Bidens bipinnata $L$.

- grandiflora Balb.

- leucantha $W$.

- macrosperma Fisch.

Biseutella apula $L$.

- erigerifolia Dec.

- eriocarpa Dec.

- lyrata $L$.

- maritima Ten.

Biserrula Pelecinus $L$.

Blitum capitatum $\boldsymbol{L}$. petiolare. virgatum $L$.

Blumenbachia insignis Schrad.

Bocconia cordata $W$.

Bonjeania microphylla Reichb.

Brachypodium asperum $R$, et Sch.

- longifolium $P . B$.

Brassica gigantea Hortul.

Briza maxima $L$.

- virens $L$

Brizopyrum siculum $L k$.

Bromus jubatus Ten.

- lanuginosus Peir.

Broteroa trinervata Pers.

Browallia elata $L$.

Bulbine annua $W$

Bunias Erucago $L$

- orientalis $L$.

Bunium peucedanoides $\boldsymbol{M}$. $\boldsymbol{B}$.

Buphthalmum grandiflorum $L$.

- maritimum $L$.

- salicifolium $\boldsymbol{L}$.

- spinosum $L$.

Bupleurum falcatum $L$.

- heterophyllum $L k$.

- multinerve Dec.

- nervosum Trev. sibiricum Trev.

Cacalia atriplicifolia $L$. sonehifolia $L$.

Cakile perennis Dec. rugosa Dec.

Calamagrostis argentea Dec

Calamintha obliqua Host. rupestris Host.

Calandrinia compressa Schrad.

- discolor Schrad. grandifiora Lindl.

Calceolaria erenatiflora Cav. pendula Don.

Caldasia heterophylla $I V$.

Calondula aegyptiaca Desf.

- maritima Guss.

- pluvialis $L$.

- stellata Cav.

Calliopsis Drummondi Hook.

Callistachys lanceolata $V$ ent.

Camelina dentata Pers.

Campanula betonicaefolia $\boldsymbol{M}$. $\boldsymbol{B}$.

- carpathica $L$.

- divergens $W$.

- Erinus $L$.

Campanula hybrida $\boldsymbol{L}$. - illinoensis Fresen.

- latifolia $L$.

- obliquifolia Ten.

- peirescifolia Fisch.

- peregrina $L$.

- pyramidalis $L$.

- urticaefolia Schmidt.

Campelia media Link.

Canna speciosa Rosc.

Capraria biflora $L$.

Capsicum bicolor Jacq.

- chinense $L$.

pendulum $W$.

Cardiospermum Halicacabum $L$

Carex divulsa Good.

- festucacea $W$

- laevigata $S m$.

- logopodioides $\boldsymbol{W}$.

- maxima Scop. nubigena Don.

Carthamus tinctorius $L$.

Carum Bulbocastanum Koch.

Catananche coerulea $L$.

Caucalis grandiflora $L$.

Celsia Arcturus $L$.

- australis.

- lanata Jacq.

- orientalis $L$.

Cenchrus echinatus $L$.

Centauren africana Lam.

- calocephala $W$.

- cicutaefolia Horn.

- Crocodilium L.

- Crupina $L$

- depressa M. B.

- eriophora $L$.

- macrocephala.

- melitensis $L$.

- moschata $L$.

- ragusina $L$

- reflexa Lam.

- rupestris $L$.

- ruthenica Lam.

- sonchifolia $L$.

- stereophylla Bess.

- sulphurea $W$.

- tatarica $L$

Centrospermum Chrysanthemum Spreng.

Cerastium davuricum Fisch.

- dichotomum $L$.

- grandiflorum Kit.

- tomentosum $L$.

Ceratocephalus falcatas Pers.

Ceratochloa pendula Schrad.

- unioloides Dec.

Cerinthe aspera Roth. major $L$.

Chaiturus Marrubiastrum Rchb.

Cheirinia crepidifolia $L k$.

Chelidonium laciniatum Mill.

Chelone eampanulnta Cav.

- diffusa Dougl.

- Digitalis Spr.

- gentianoides.

- hirsuta $L$.

- ovata Dougl. 
Cinna expansa Link.

Cirsium canum $M$ : $B$.

- laniflorum M. B.

- scleranthon $\boldsymbol{M} . \boldsymbol{B}$.

Cistus candidissimns Dun.

- monspeliensis $L$.

- vaginatus Ait.

Clarkia elegans Dougl.

- pulchella Prersh.

Claytonia perfoliata Don.

Clematis angustifolia Jacq.

- campaniflora Brot.

- lathyrifolia Bess.

Cleome graveolens Rafin.

- spinosa $L$.

- violacea $L$

Clinopodium atropurpurenm

Clintonia elegans Dougl. H. Gryph.

Cneorum tricoccon $L$.

Cnicus syriacus $W$.

- tuberosus.

Cnidium apioides $S p r$.

- venosum Koch.

Coix exaltata Jacq.

Collinsia bicolor Benth.

Collomia coceinea Lehm.

- grandiflora Dougl.

Colpodium Steveni Trin.

Commelina angustifolia $M x$.

- clandestina Mart.

- coelestis $L$

- Karwinskii Nart.

- polygama Roth.

Conium maculatum $\beta$. sibiricum.

Conringia perfoliata $L k$.

Convolvulus siculus $\boldsymbol{L}$.

Corchorns hirsutus $\boldsymbol{L}$.

- olitorins $L$.

- trilocularis $L$.

Coreopsis Atkinsoniana Dougl.

- lanceolata $L$.

- praecox Fresen. 1)

Coriaria myrtifolia $\boldsymbol{L}$.

Corispermum canescens Kit.

Coronilla coronata $L$.

- glanca $L$.

- Securidaca $L$

Corydalis aurea $\boldsymbol{W}$.

- nobilis Pers.

- sempervirens Pers.

Crambe grandiflora Stev.

- maritima $\boldsymbol{L}$.

Crepis heterosperma Schrad.

- lacera Tenor.

Crotalaria sagittalis $\boldsymbol{L}$.

Crucianella angustifolia $\boldsymbol{L}$.

Cucabalus bacciferus $L$. catholieus $\boldsymbol{L}$.

Cucurbita lagenaria $L$.

Caphea viscosissima Jacq.

Cyclachaena xanthifolia Fresen.

Cynanchum fuscatum Link,

- nigrum Pers.

Cynoglossum apenninum $L$.

- bicolor $W$.

- canescens $W$.

- sylvaticum $\boldsymbol{S} m$.

Cyperus alternifolins $L$.

- epectabilis Schreb.

Cytisus capitatus Jacq.

- hirsutus $L$.

- prostratus.

Dactylaena micrantha Schrad.

Daphne Laureola $\boldsymbol{L}$.

- Mezereum $\boldsymbol{L}$.

Datisca cannabina $\boldsymbol{L}$.

Datura Tatula $L$.

Daucus hispidus Desf.

- maritimus $L$.
Daucus muricatus $\boldsymbol{L}$.

Decanenrum senegalense $D e c$.

Delphinium grandiflorum $\boldsymbol{L}$.

- montanum Dec.

- Requienii Dec.

Staphisagria $\boldsymbol{L}$.

Desmanthus virgatns $W$

Dianthus collinus Kit.

- diutinns Kit.

Dictamnus albus $L$,

Didiscus caeruleus Hook.

Digitalis Buxbaumii Bess.

- ferruginea $\boldsymbol{L}$.

- grandiflora Lam.

- laevigata Kit.

- lanata Ehrh.

- lutea L.

- Sceptrum $L$.

Digitaria aegyptiaca $W$.

Dinebra curtipendula Dec.

Diplachne fascicularis $P$. B .

Diplophyllum veronicaeforme $L \mathrm{ehm}$.

Dipsacus laciniatus.

Dorycnium herbaceum Vill.

Draba aurea Vahl.

- contorta Ehrh.

- fladnizensis $W u l f$.

- nummularia Ehrenb.

Dracocephalum canariense $\boldsymbol{L}$.

- canescens $L$.

- Moldavica $\boldsymbol{L}$.

- peltatum $L$.

- sibiricum $L$.

- thymiflorum $\boldsymbol{L}$.

Eccremocarpus scaber $\boldsymbol{R}$. et $\boldsymbol{P}$.

Echinacea purpurea Moench.

- serotina Dec.

Echinocactus Sellovii.

Echinops Ritro $\boldsymbol{L}$.

- sphaerocephalus $L$.

Eclipta erecta $\boldsymbol{L}$.

- Iatifolia $\boldsymbol{L}$.

Eleusine coracana Pers.

- indica Lam.

- rigida $S p r$.

- Tocussa Fres.

Elichrysum bracteatum Vent.

- fulgidum $\boldsymbol{W}$.

Elsholtzia cristata $W$.

Elymus arenarius $\boldsymbol{L}$.

- canadensis $\boldsymbol{L}$.

- carolinianus.

- giganteus Vahl.

Emex spinosus Camp.

Entelea arborescens $\boldsymbol{R} . \boldsymbol{B r}$.

Epilobium angustissimum Ait.

Eragrostis abyssinica $L k$.

Eranthis hyemalis Salisb.

Eremogone graminea C. A. Mey.

Erigeron contortus Desf.

- elengatus Led.

- glabellus Nutt.

- podolicus Bess.

- purpureus Ait.

- Villarsii Bell.

Erinns alpinns $L$.

Erodium gruinum Ait.

- melanostigma Mart.

- moschatum Ait. pulverulentum $W$.

Ervum agrigentinum Guss.

- Ervilia $L$.

Erysimum altaicum Meyer.

duhium Dec.

- hieracifolium.

- odoratum Ehrh.

Esehscholzia californica Cham.

Euphorbia cyathophora Jacq.

- Humboldtii $\boldsymbol{W}$.

Lagascae Spr.
Enphorbia Lathyris $L$.

- prunifolia Jacq.

Eustachys petraea Desv.

Eutoca viscida Benth.

- Wrangeliana $F$, et $M$.

Exacum viscosum $S m$.

Farsetia clypeata $\boldsymbol{R}$. $\boldsymbol{B r}$.

- eriocarpa Dec.

Fedia Cornucopiae Vahl.

Ferula campestris Bess.

- Ferulago L.

Forskolea viridis Ehrenb.

Francoa appendiculata Cav. - sonchifolia $S p r$.

Fuchsia arborescens Sess.

- coccinea $L$.

Fumaria capreolata $\boldsymbol{L}$.

Galaidia bicolor Lam.

Galega persica Pers.

- strieta Ait.

Galinsogea trilobata Cav.

Gasteria obliqua Haw.

Gaura biennis $\boldsymbol{L}$

- mutabilis Cav.

- tripetala Cav.

Gentiana asclepiadea $\boldsymbol{L}$.

- cruciata $L$.

- septemfida Pall.

Geranimm earolinianum $\boldsymbol{L}$.

- sanguineum $L$.

Geropogon glaber $\boldsymbol{L}$.

Geum album Gmel.

- atlanticum Desf.

- coccinenm Sm.

- heterophyllum.

- japonicum Thunb.

- macrophyllum $W$.

- ranunculoides $S e r$.

Gilia achilleaefolia Benth.

- capitata Hook.

- inconspicua Hook.

- laciniata $\boldsymbol{R}$. et $\boldsymbol{P}$.

- tricolor Benth.

Gladiolus psittacinus Hook.

Glaucium lutenm Scop.

- violaceum $\boldsymbol{S} m$.

Glycine Comptoniana Andr.

Glycyrrhiza echinata $\boldsymbol{L}$.

- foetida Desf.

- glabra $L$.

- glandulifera Kit.

Gnaphalium foetidum $\boldsymbol{L}$.

Goodenia grandiflora Sims.

Grahamia aromatica Hook.

Grindelia incisa $\mathbf{S p r}$.

Gypsophila acutifolia Fisch.

- altissima $L$.

- collina Stev.

- elegans $M$. B.

- glauca Stev.

- repens $L$.

- Rokejeka Del.

- Steveni Fisch. stricta Bunge.

Hablizia tamnoides $M$. B.

Haloragis Cercodia Ait.

Hasselquistia cordata $\boldsymbol{L}$.

Hedypnois pendula $W$.

Hedysarum alpinum $L$

- canadense $\boldsymbol{L}$.

- elongatum Fisch.

- flexuosmm $L$. sibirieum Lam.

Heimia myrtifolia $L k$ salicifolia $L k$.

Helenium autumnale $\boldsymbol{L}$

- californicum Dougr.

- mexicanum Humb.

Helianthemum aegyptiaeum Mill.

- racemosum Pers.

Helianthus lenticularis Lindl.

Heliotropium europaeum $\boldsymbol{L}$.

Hemerocallis graminea $A n d r$.

Hemimeris urticaefolia $W$.

Heraclenm angustifolium $\boldsymbol{L}$.

- asperum M. $\boldsymbol{B}$.

- dissectum Led.

- Panaces $L$.

- speciosum.

- villosum Fisch.

Hermannia denudata $L$.

Heterospermnm pinnatum Cav.

Heuchera americana $L$.

Hibiscus Manihot $L$.

- pedunculatus Thunb.

- radiatus Cav.

- Richardsonii Sweet.

- syriacus $L$.

- Trionum $L$

- $\quad$, $\beta$. ternatus Cav

Hiorthia "urea Less.

Hippocrepis ciliata $W$.

- mnltisiliqua $\boldsymbol{L}$.

- unisiliquosa $\boldsymbol{L}$

Holoschoenus atrovirens $L k$.

Humea elegans $S \mathrm{~m}$.

Hydrocotyle silthorpioides Lam.

Hyoscyamus aureus $L$.

- canariensis $\mathrm{Ker}$.

- physaloides $L$. pusillus $L$.

Hyoseris arenaria Schousb.

- seabra $L$

- tubaeformis Ten.

Hypecoum procumbens $L$.

Hypochoeris arachnites Biv.

Hyssopus angustifolius $\boldsymbol{M}$. $\boldsymbol{B}$.

- Foeniculum Spr.

- Lophanthus $\boldsymbol{L}$.

- nepetoides $L$.

- officinalis $\boldsymbol{L}$.

- orientalis $\boldsymbol{W}$ scrofularifolius $W$.

Impatiens parviflora Dec.

Indigofera Anil $\boldsymbol{L}$.

- australis $W$.

divaricata Jacq.

frutescens Thunb.

Inula bifrons $\boldsymbol{L}$.

- germanica $L$.

- odora $L$.

- suaveolens Jacq. 
Kochia scoparia Schrad.

Kuhnia suaveolens. ${ }^{2}$ )

Lactuca crispa Roth.

- perennis $\boldsymbol{L}$.

Lagoecia cuminoides $\boldsymbol{L}$.

Lamarckia aurea Mönch.

Lantana salviaefolia Jacq.

Lappago racemosa Schreb.

Lapsana grandiflora $\boldsymbol{M}$. $\boldsymbol{B}$.

Laserpitinm angustifolium. - gallicum $L$ - pilosum $\boldsymbol{W}$.

Lasiopetalum purpurenm Sims.

Lasiospermum radiatum Trev.

Lathyrus angulatus $\boldsymbol{L}$.

- annuus $L$.

- Aphaca $L$

- Clymenum $\boldsymbol{L}$.

- latifolius $L$

- longipedunculatus Led.

- magellanicus Lam.

- mexicanus Wender.

- Nissolia $\boldsymbol{L}$.

- Ochrus Dec.

- speciosus $L$.

Lavandula latifolia $\mathrm{Ehrh}$.

- Stoechas $\boldsymbol{L}$.

Lavatera arborea $\boldsymbol{L}$. plebeja Sims.

Leontodon apenninus Ten.

Leonurus nepalensis.

Lepidium affine Led. suffruticosum $L$.

Lepidonema chilensis Fisch, et $\boldsymbol{M}$.

Lessertia perennans Dec.

Leucocarpus alatus $D_{0}$.

Levisticum officinale Koch.

Liatris pycnostachya Michx. - scariosa $W$. spicata $W$.

Libanotis Buchtormensis Dec.

- villosa Turcz. vulgaris $D_{e c}$.

Ligusticum alatum Spr.

- Seguieri Koch.

Linaria bipartita $W$.

- genistaefolia Mill.

- litoralis Bernh.

- purpurea Mill.

- pyrenaica Dec.

- triphylla $\boldsymbol{W}$.

Lindenbergia urticaefolia Lehm.

Linum flavum $\boldsymbol{L}$.

- narbonense $L$.

- sibiricum Dec.

- suffruticosum $L$.

Lobelia alata $\boldsymbol{R} . \boldsymbol{B} \boldsymbol{r}$.

- inflata $\boldsymbol{L}$.

- syphilitica $\boldsymbol{t}$.

- triquetra $L$.

Lolium speciosum Stev.

Lomatophyllum borbonicum $W$.

Lopezia coronata Andr.

Lophospermum scandens Don.

Lotus edulis $\boldsymbol{L}$.

- Jacobaeus $\boldsymbol{L}$.

- ornithopodioides $\boldsymbol{L}$.

- siliquosus $L$.

Lupinus allous $\boldsymbol{L}$.

- angustifolius $\boldsymbol{L}$.

- bicolor.

- Cruikshankii Bot. Mag. hirsutus $L$.

- Lehmanni Müll.

- linifolius Roth.

lituratus Reichb.

luteus $L$.

- micranthus Dougl.

- pilosus $\boldsymbol{L}$.

- polyphyllus Dougl.
Lupinus pulehellus Dougl. - succulentus Lindl.

- Termis Forsk. omentosus Sweet.

Luzula maxima $D e c$.

Lychnis Coeli rosa Desr.

- fulgens Fisch.

Lycopus exaltatus $L$. virginicus $L$.

Lysimachia ciliata $L$.

- dubia Ait.

- Ephemerum L.

- verticillata Pall.

Lythrum verticillatum $\boldsymbol{L}$.

- virgatum $L$.

Madia elegans Don. quinqueradiata Fisch. et $M$.

Malcolmia africana $R, B r$.

- arenaria Dec.

- maxitima $\boldsymbol{R}$. $\boldsymbol{B r}$.

Malope grandiflora Hort.

- malacoides $\boldsymbol{L}$.

Mandragora officinalis Mill.

Mariscus paniceus $V a h l$.

Marrubium africanum $\boldsymbol{L}$.

- astracanicam Jacq.

- parviflorum $F$. et $M$. propinqum $F$. et $M$.

Maurandia Barklayana Lindl. semperflorens Jacq.

Meconopsis cambrica $V i g$.

Megastachya rigida Beauv.

Melica altissima $L$.

- ciliata $L$.

Melilotus caerulea Desv.

- var. connata.

- indica Desv.

- rotundifolia Ten.

- rugulosa $W$

- ruthenica $M . B$.

Melissa alba Kit.

Menispermum canadense $\boldsymbol{L}$. [me $L$.

Mesembrianthemum pugionifor-

Meum athamanticum Jacq.

Mimulus cardinalis Lindl.

- floribundus Lindl.

- Inteus $L$.

- moschatus Dougl.

- roseus.

Mirabilis dichotoma $\boldsymbol{L}$.

- Jalappa $\boldsymbol{L}$.

- longiflora $L$.

Mogiphanes virgata Schrad.

Molinia altissima Link.

Mollia diffusa $\boldsymbol{W}$.

- latifolia $W$

Moluccella laevis $L$.

Momordica Elaterinm $\boldsymbol{L}$.

Moraea chinensis Thunb.

Mühlenbergia diffusa Schreb.

Muscari comosum $\boldsymbol{W}$.

Myagrum perfoliatum $\boldsymbol{L}$.

Myosotis peduncularis Trev. - sparsiflora Mikan.

Myrrhis aromatica Spreng. odorata Scop.

Nemophila Atomaria $F$. et $M$.

- insignis Dougl.

- pedunculata Dougl.

- phacelioides Bart.

Nepeta coerulea Ait.

- colorata $W$.

- fissa C. A. Mey.

- grandiflora.

- italiea $L$.

- longiflora Vent.

- marrubioides $\boldsymbol{W}$

- pannonica Jacq.

- teucrioides Lam.
Nepeta ucranica $L$.

Nicandra physalodes Gaertn.

Nicotiana alata $L k$. et Otto,

- chinensis Fisch.

- fruticosa $L$.

- glanca Hook.

- glutinosa $L$.

- Langsdorffii Nees.

- maerophylla Spr.

- noctiflora Hook. vineaeflora $\mathrm{Lag}$.

Nigella hispanica $L$.

- orientalis $L$.

- sativa $L$.

Nonea pulla Dec.

Obeliscaria pinnata Cass.

Oenothera chilensis.

- cruciata Nutt.

- Fraseri Pursh.

- fruticosa $\boldsymbol{L}$.

- glauca Mx.

- Lindleyana Sims.

- muricata $L$.

- odorata Jacq.

- Romanzovii Led.

- rosea Ait.

- Simsiana Ser.

- spectabilis fforn. triloba Nutt.

Omphalodes linifolia Moench.

Onobroma creticnm Spr.

- lanatum $S p r$.

Onobrychis Caput galli Lam.

- conferta $s p r$.

- Crista galli Lam.

Ononis alopecuroides $L$

- fruticosa $L$.

- hircina.

Natrix $L$.

Onopordon illyricum $L$.

- macracanthum Schousb. iscosum.

Ornithogalum collinum Guss. narbonense $L$.

Orobus atropurpureus Desf.

- niger $L$

- venosus $W$

- vernus $\boldsymbol{L}$.

Oxyria reniformis Hook.

Paeonia humilis Retz.

- officinalis $L$.

- peregrina Mill.

- tenuifolia $\boldsymbol{L}$

villosa.

Papaver bracteatum Lindl.

- hybridum $L$.

- intermedium Alph. Dec.

- orientale $L$

- somniferum $\beta$. album.

- spectabile $\boldsymbol{H}$. Ber.

- tauricum.

Paspalum elegans Flügg.

- serobiculatum $\boldsymbol{L}$.

- stoloniferum Bosc.

Passiflora edulis Sims.

- graeilis Jacq.

- heterophylla.

- Raddiana Dec.

Pastinaca pimpinellifolia $M$. $B$.

Pavonia praemorsa $\boldsymbol{W}_{\text {. }}$

Peganum Harmala $L$.

Pelargonium malvaefolium Jacq.

Periptera punicea Dee.

Petiveria alliacen $L$.

Peucedanum baicalense $K$ och.

- involueratum $\boldsymbol{K o c h}$.

- terebinthinaceum $F i s c h$.

- verticillare $K o c h$.

Phaca alpina $J a c q$.

Phacelia bipinnatifida $M \boldsymbol{s}$.

Phacelia tanacetifolia.

Phleum asperum Vill.

Phlomis salviaefolia.

- tuberosa $L$.

Phyllanthus juglandifolius $W$.

Phyllis Nobla $L$.

Phyllolobium chinense Fisch

Physalis flexuosa $L$.

Phyteuma Scheuchzeri $A l l$.

- strictum Ker.

- virgatum $W$.

Phytolacea decandra $\boldsymbol{L}$.

Picridium tingitanum Desf.

Picris altissinu $\mathrm{Del}$.

strigosa $M . B$.

Pimelea drupacea Labill.

Pimpinella peregrina $L$.

Pisum sativum $\mathbf{L}, \gamma$ umbellatum

Pittosporum undulatum Andr.

Plumbago mierantha Desf.

Pocockia cretica Ser.

Podalyria australis $\boldsymbol{W}$.

Polemonium coeruleum $I F$

Polygonum cymosum Trev.

- emarginatum Roth.

- undulatum Murr.

Pomaderris elliptica Lab.

- phylicaefolia $L k$.

otentilla arguta Pursh.

- atrosanguinea Don.

- calabra Ten.

- formosa Don.

- gracilis Dougl.

- ontopoda Lehm.

- recta $L$.

- rupestris $L$.

- Thomasii Ten.

- viscosa Don.

Poterium garganicum Ten.

- guestphalicum Bönn. spinosum $L$.

Primula villosa $J_{a c q}$.

Prismatocarpos falcatus Ten.

Prunella alba Pall.

- grandiflora $L$.

- hyssopifolia $L$.

- pensylvanica $\boldsymbol{W}$

Psoralea bituminosa $L$.

- bracteata $\boldsymbol{L}$.

- capitata Thunb.

- EckIoni Ott.

- hirta $L$. 
Ribes aureum Pursh.

Richardia scabra $\boldsymbol{L}$.

Rivina laevis $L$.

Rosa rubifolia $R$. $B r$

Rudbeckia amplexifolia Jacq.

- laciniata $\boldsymbol{L}$.

- speciosa Wender. triloba $L$.

Ruellia capensis.

Ruta chalepensis $\boldsymbol{L}$.

Salsola baccata Poir. Tragns $L$.

Sambucus canadensis $\boldsymbol{L}$.

Samolus Valerandi $\boldsymbol{L}$.

Sanicula marilandica $L$.

Sanvitalia procumbens $\boldsymbol{W}$.

Saponaria calabrica Guss.

- ccrastoides Led.

- Vaccaria $L$.

Saracha viscosa Schrad.

Satureja montana $\boldsymbol{L}$.

Saxifraga dentata $L k$.

- pyramidalis Lap.

- rotundifolia $L$.

Scandix brachycarpa Giuss.

Schivereckia podolica Andrz.

Schizanthus pinnatus $R$, et $P$.

- retusus Hook.

Stoparia dnlcis $L$.

Scorpiurns muricata $\boldsymbol{L}$.

- sulcata $L$.

- vermiculata $L$.

Scrofularia Balbisii Horn.

- cordata Pers.

- mellifera Ait.

- peregrina $L$.

- Scorodonia L.

- variegata $M . B$.

- vernalis $L$

Scutellaria Iateriflora $L$.

- pallida $M . B$.

- peregrina $L$.

- rubicunda Horn.

Secale fragile $\boldsymbol{M}$. $\boldsymbol{B}$.

Sedum altaicum Bess.

- hybridum $\boldsymbol{L}$.

- Rhodiola Dec.

Selago faseiculata $\boldsymbol{L}$.

Senecio Doria $L$.

- elegans $L$.

- ovatus $W$.

- rapistroides Dec.

Seriola aethnensis $L$.

- glauca Tin.

Serratula alata $W$
Serratula radiata $M$. B.

Seseli glaucum $\boldsymbol{L}$.

- gummiferum $\boldsymbol{S m}$.

- tortuosum $\boldsymbol{L}$

Sesleria coerulea $\mathrm{Ard}$.

- elongata Host. nitida Ten.

Setaria italica Kunth.

purpurascens $\boldsymbol{H}$. et $\boldsymbol{K}$.

Sida Abutilon $\boldsymbol{L}$.

- angustifolia Juss.

- indica $\boldsymbol{L}$.

- Napaea Cav.

- triloba Cav.

- vesicaria Cav.

Sideritis montana $\boldsymbol{L}$.

Siegesbeckia flosculosa Herit.

- orientalis $L$.

Silene alpestris Jacq.

- chlorantha Ehrh.

- compacta Horn.

- cretica $\boldsymbol{L}$.

- flavescens $K i t$.

- fruticosa $L$.

- gallica $L$.

- hirsuta Lag.

- imbricata Desf.

- inamoena Lindl.

- infracta Kit.

- linaccola $W i b$.

- livida $\boldsymbol{W}$.

- mollissima $\boldsymbol{S} m$.

- pendula $L$.

- quinquevulnera $\boldsymbol{L}$

_ regia Sims.

- saponariaefolia Schott.

- sericea All.

- viridiflora $L$

Sinapis auriculata $\mathrm{Dec}$.

Sisymbrium austriacnm Jacq.

- strictissimum $\boldsymbol{L}$.

Sisyrinchium Bermudiana $\boldsymbol{L}$.

- striatum Cav.

Sonchus flexuosus Ledeb.

- floridanus $\boldsymbol{L}$.

- leucophaeus $\boldsymbol{W}$.

- palhstris $\boldsymbol{L}$.

- setosus Ehrenb.

Sorghum cernnum $W$.

Spartina cynosuroides Roth.

Spartium junceum $\boldsymbol{L}$.

Specularia biflóra $\boldsymbol{F}$, et $\boldsymbol{M}$.

Spermacoce longifolia $\mathrm{Aubl}$.

Stachys alpina $\boldsymbol{L}$.
Stachys cretica $\boldsymbol{L}$.

- grandiflora Host.

- iberica $M$. B.

- lanata Jacq.

- polystachya Ten.

- setifera C. A. Mey.

Stachytarpheta jamaicensis $\mathrm{Vahl}$.

Stenactis speciosa Lindl.

Stipa capillata $\boldsymbol{L}$.

- gigantea Lag.

Stylidium adnatum $\boldsymbol{R}$. $\boldsymbol{B r}$.

Sutherlandia frutescens $\boldsymbol{R}, \boldsymbol{B}$.

Swainsonia coronillaefolia Salisb.

Symphoria racemosa Pursh.

Syncephalantha decipiens Bartl.

Syrenia Biebersteinii Andrz.

Tanacetum boreale.

Teedia lucida Rud.

Telekia cordifolia Kit.

Tellima grandiflora Lindl.

Teloxis aristata Moq. Tand.

Tetrapoma barbareaefolium Turcz.

Teucrium Arduini $\boldsymbol{L}$.

- flavum $L$.

- hyrcanicum $L$.

- massiliense $L$.

- Psendo-Scorodonia Desf.

Thalictrum aquilegifolium $L$.

Thrincia hirta Rolh.

Tiaridium indienm Lehm.

Tolpis barbata Gaertn.

Tordylium apulum Riv.

- maximum $L$.

syriacum $L$.

Torenia scabra $\boldsymbol{R}$. $\mathrm{Br}$.

Trachynia distachya Link.

Tradescantia erecta Cav.

- Selloviana Pohl.

Tragopogon eriospermus Ten.

- floceosus Kit.

- porrifolius $L$.

Tribulns terrestris $L$.

Trichodinm laxiflorum Mich.

Tricholaena rosea Nees.

Trifolium bracteatum Schousb.

- incarnatum $L$.

- libanoticum Ehrenb.

- ligusticum Balb.

- subterraneum $\boldsymbol{L}$.

- vesiculosum Sant.

Trigonella arcuata Meyer.

- Calliceras Fisch.

- cancellata Fisch.

- Foenum graecum $L$.

- tenuis Fisck.
Trigonella uralensis Led.

Triticum dasyanthon Ledeb.

Triumfetta oblongata $L k$.

Urachne paradoxa $L k$.

Urospermum Dalechampii Desf. picroides Desf.

Urtica cannabina $\boldsymbol{L}$.

- convexa Horn. pilulifera $\boldsymbol{L}$.

Valerianella coronata Dec.

- hamata Dec.

- uncinata Dufr.

Verbaseum condensatum Schrad. orientale $\boldsymbol{M}$. $\boldsymbol{B}$.

Verbena Aubletia Jacq.

- bonariensis $L$.

- bracteosa $M x$.

- hastata $L$.

- lasiostachys $L k$.

- paniculata Lam.

- strieta Vent.

- urticaefolia $L$.

- venosa.

Vernonia anthelminthiea $W$

Veronica brevifolia $M . B$

- caucasiea M. B.

- prostrata $L$.

- virginica $L$.

Vesicaria cretica Poir.

- sinuata Poir.

Vicia articulata $L$

- biennis $L$.

- bithynica $L$.

- dasyearpa Ten.

- globosa Retz.

- Iutea $L$.

- Nissoliana Gouan.

Viminaria denudata $S m$.

Viola hederacea Labill.

Wahlenbergia grandiflora Schrad.

Xanthium orientale $L$.

- spinosum $\boldsymbol{L}$.

Ximenesia encelioides Cav.

Zacyntha verrucosa Gärtn.

Zephyranthes candida Herb.

Zinnia elegans var. coccinen.

- tenuiflora Jacq.

- verticillata $A n d r$.

Zizia aurea Koch.

Ziziphora dasyantha $M . B$.

- serpyllacea.

Zoegea Leptaurea $\boldsymbol{L}$.

Zozimia absinthifolia Dee.

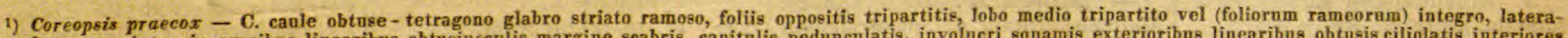

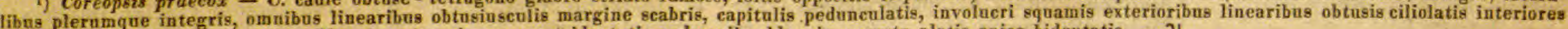
sbaequantibue, ligulis otevato - oblongis apice plerumque tridentatis, achaeniis oblongis anguste alatis apice bidentatis. -24.

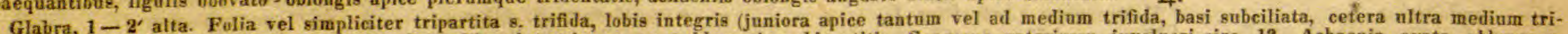

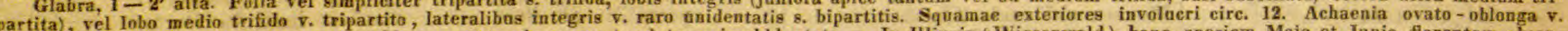

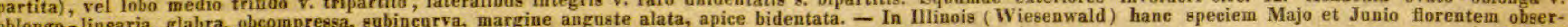
vavit Dr. Excriarsas.

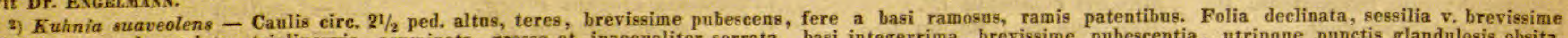

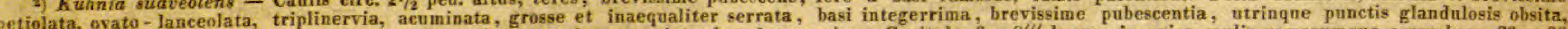

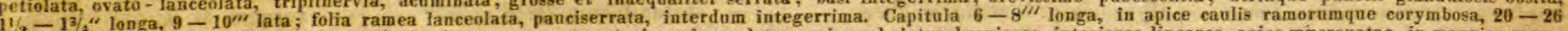

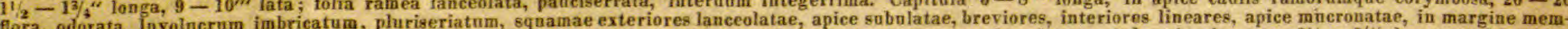

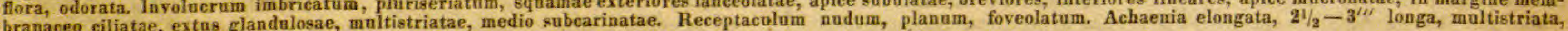
branaceo ciliatae, extus glandulosae, multist

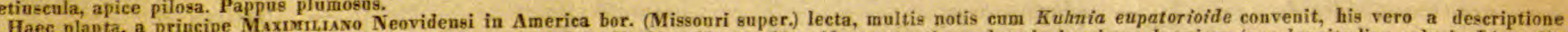

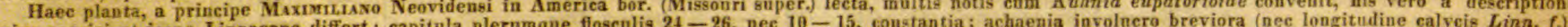

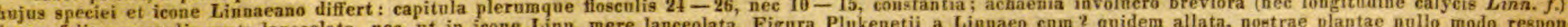

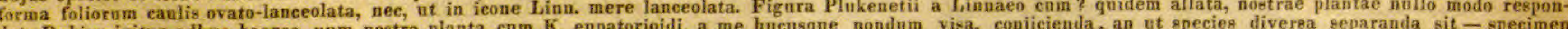

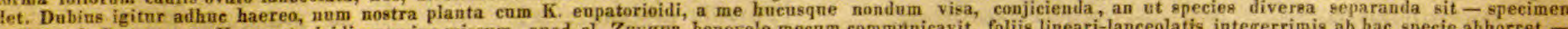

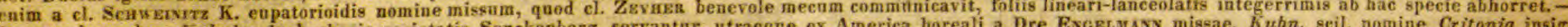

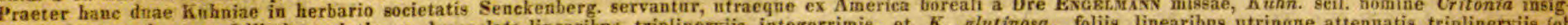

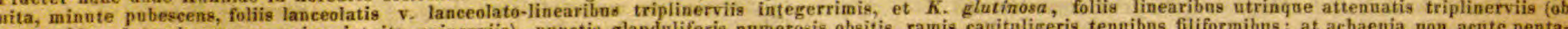

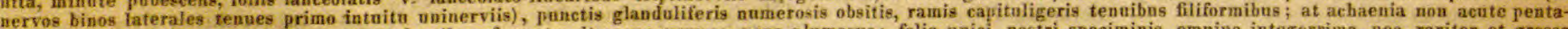

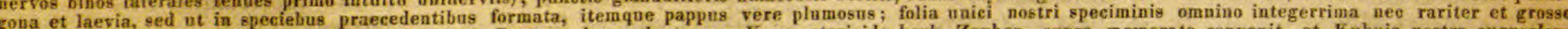

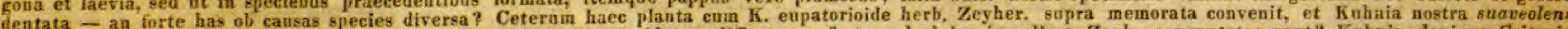

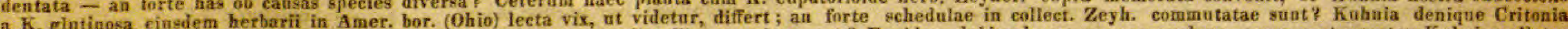

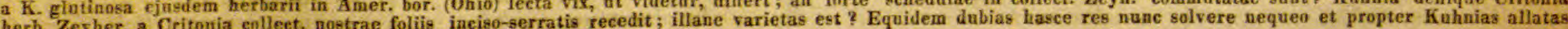
herbaria accurate inspieienda esse puto.

G. Fregentus.

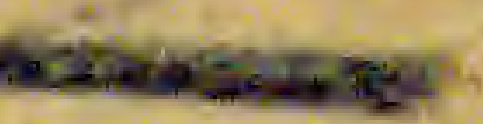


Acacia armata $\boldsymbol{R} . \boldsymbol{B r}$.

- Farnesiana $W$.

- Julibrissin $\boldsymbol{W}$.

- leucocephala Berter.

- verticillata $W$.

Achillea Ageratum $L$.

- albicaulis C. A. Mey.

- alpina $\boldsymbol{L}$.

- biserrata M. B.

- magna $L$.

- myriophylla $\boldsymbol{H}$.

- ochroleuca.

- odorata $L$

- pubescens $L$.

- setacea $\boldsymbol{W}$. Kit.

- sylvatica.

tanacetifolia All.

Achyranthes axillaris $W$.

- brachiata $L$.

- fruticosa Lam.

- porrigens Jacq.

Achyropappus schkuhrioides $L k$.

Acroglochin chenopodioides Schrd.

Actaea brachypetala Dec.

- racemosa $L$

Actinomeris alternifolia $D e c$.

- oppositifolia Fresen.

Adenocarpus foliolosus Dec. intermedius.

Adenophora communis Fisch.

- suaveolens Fisch.

Adenophyllum coccineum Pers.

Adlumia cirrhosa Rafin.

Adonis aestivalis $\boldsymbol{L}_{\text {. }}$ ? antumnalis $L$ vernalis $L$

Aegilops cylindrica Host.

- ovata $L$.

- squarrosa $L$.

Aethionema eappadocicum Spr. saxatile $R$. $B r$

Agrimonia odorata Mill.

- pilosa Led.

Agrostemma pusilla Hort. Paris.

Agrostis elegans.

- lachnantha Nees ab Es.

- mexicana $L$.

Ajuga Chamaepitys Schreb.

Aldeaea pinnata $R$. et $P$.

Allium acutangulum $W$.

- albidum Fisch.

- atropurpureum Kit.

- azureum Led.

- baicalense $\boldsymbol{H}$

- fragrans Vent.

- neapolitanum Cyrill.

- ramesum Jacq.

- sibiricem $L$.

- striatum Jacq.

- tenuiflorum Ten.

- triquetrum $L$.

- Vietorialis $L$.

Alstroemeria Pelegrina $L$.

Althaea Froleviana Fisch.

- Sieberi $L k$.

Alyssum saxatile $\boldsymbol{L}$.

Amarantus speciostis $\mathrm{Ker}$.

- spinosus $\boldsymbol{L}$.
Ambrosia trifida $L$

Amethystea coerulea $L$.

Ammi majus $L$.

Ammobium alatum $R$. $B r$

Amsinkia speetabilis Fisch. Mey.

Amsonia angustifolia $M x$.

Anacyclus bicolor.

Pyrethrum $L k$.

Anchusa Barrelieri Gmel.

- hybrida Ten.

- ochroleuca $M . B$.

- paniculata Ait.

- sempervirens $L$.

Andropogon stenophyllus $R$. et Sch.

Androsace maxima $L$.

- septentrionalis $L$.

Androsaemum offieinale $A l l$.

Andryala cheiranthifolia Herit. lanata $\boldsymbol{L}$.

varia Low.

Anemone canadensis $\boldsymbol{H}$. Bon. Hudsoniana Richards. ochotensis Fisch.

Anethum Sowa Roxb.

Angelica decurrens Led.

Anisodus luridus Link.

Anoda hastata Cav.

Anomatheca cruenta Lindl.

Anthemis nobilis $L$.

- rigescens $W$. ruthenica M. B. secundiramea Biv,

Antherienm Liliago $L$.

- Liliastrum $\boldsymbol{L}$.

Anthyllis tetraphylla $L$.

Antirrhinum elegans Pers. sempervirens Lap. siculum $U \mathrm{cr}$.

Aquilegia canadensis $\boldsymbol{L}$.

- elata Led.

- glandulosa Fisch.

- sibirica Lam. speciosa H. Marb.

Arabis alpina $L$.

- bellidifolia $L$.

- collina Ten.

- crispata $W$

- lilacina Schrad.

- pendula $\boldsymbol{L}$.

- rosea Dec.

Arachis hypogaea $L$.

Arenaria calycina $P_{0}$ oir rigida $M$. $B$. rostrata $K$ it.

Argemone Barklayana Grah. mexicana $\beta$. albiflora.

Artemisia Sieversiana Fhrh. violacea Desf.

Arthropodium cirratum $R . B r$.

- minus $R$. $B r$. pendulum $\mathrm{spr}$

Asclepias angustifolia $W$.

- curassavica $L$

- incarnata $L$.

- nivea $L$.

Asperula mostana Kit.

Asphodelus albus $\boldsymbol{W}$.

- clavatus Roxb.
Asphodelus creticus Lam.

- Inteus $L$.

- tanricus $M . B$.

- tenuior M. B.

Asprella Hystrix $\boldsymbol{W}$.

Aster Amellus $L$.

Astragalus alopecuroides $\boldsymbol{L}$.

- asper Jacq.

- baeticus $L$

- brachyceras Led.

- carolinianus $L$

- Cicer L.

- cymbaeformis Brot.

- galegiformis $\boldsymbol{L}$.

- pentaglottis $\boldsymbol{L}$

- uliginosus $L$. utriger Pall.

Astrantia major $\boldsymbol{L}$.

Athamanta flexuosa Desf.

- Matthioli $W u l f$.

Athanasia annua $\boldsymbol{L}$.

Anbrietia deltoidea Dec.

Anlacospermum cuneatum Led.

Avena planiculmis Schrad.

Baccharis ivaefolia $L$

Barbarea praecox $\boldsymbol{R}$. $\mathrm{Br}$.

Basella rubra $L$.

Beckmannia erucaeformis Host.

Beta foliosa Fhrenb.

- orientalis Roth.

Betonica grandiflora $W$.

- incana Ait.

- orientalis $L$.

Bidens bipinnata $L$

- grandiflora Balb.

- leucantha $\boldsymbol{W}$.

- macrosperma Fisch.

Biscutella apula $L$

- erigerifolia Dec.

- eriocarpa Dec.

- lyrata $L$.

- maritima Ten.

Biserrula Pelecinus $L$.

Blitum capitatum $\boldsymbol{L}$.

- petiolare.

- virgatum $L$.

Bonjeania microphylla Reichb,

Botryadenia Gmelini Fisch. et Mey.

Brachypodium asperum $R$, et $S c h$.

- longifolium $P . B$.

Brassica gigantea Hortul.

Briza maxima $L$.

- virens $L$.

Brizopyrum sieulum $L k$.

Bromus jubatus $T$ en.

- lanuginosus Poir.

Broteroa trinervata Pers.

Browallia elata $L$.

Bulbine annua $W$.

Bunias Erucago $L$. orientalis $L$

Bunium peucedanoides $M$. $B$.

Buphthalmum grandiflorum $L$.

- maritimum $L$. spinosum $\boldsymbol{L}$.

Bupleurum falcatum $L$.

- heterophyllum $L k$.

- multinerve Dec.
Bupleurum nervosum Trev.

- sibiricum Trev.

Cacalia atriplicifolia $\boldsymbol{L}$.

- sonchifolia $L$

- suaveolens $L$.

Cakile perennis Dec.

- rugosa Dec.

Calamagrostis argentea $\mathrm{Dec}$

Calamintha obliqna Host.

- rupestris Host.

Calandrinia compressa Sclirarl.

- discolor Schrad.

- glandulosa Poepp.

- grandiflora Lindl.

- micrantha Schlecht.

Caldasia heterophylla $H$.

Calendula cristata Guss.

- maritima Guss.

stellata Cav.

Calimeris altaica Nees.

Calliopsis Drummondi Hook.

Callistachys lanceolata $V$ ent.

Camelina dentata Pers.

Campanula betonicaefolia $\boldsymbol{M} . \boldsymbol{B}$. carpathica $\boldsymbol{L}$. divergens $W$. Erinus $L$,

- hybrida $L$

- latifolia $L$.

- obliquifolia Ten.

- peirescifolia Fisch.

- peregring $L$.

- m $\Rightarrow+40$

- pyrticasime vigump

Canna speciosa Rose.

Capraria biflora $L$

Capsicum bicolor Jacq. chinense $L$, pendulum $\boldsymbol{W}$

Cardiospermum Halicacabum

Carex divulsa Good.

- logopodioides $W$.

Carlina acaulis $\boldsymbol{L}$.

Carthamus tinctorius $L$.

Carum Bulbocastanum Koch.

Cassinia spectabilis $\boldsymbol{R}$. $\boldsymbol{B r}$.

Cauealis grandiflora $\boldsymbol{L}$.

Celosia argentea $L$

- castrensis $\boldsymbol{L}$.

Celsia Areturus $L$.

- anstralis.

- lanata Jacq.

- orientalis $L$

Cenchrus echinatus $L$

Centanrea africana Lam.

- benedicta $L$.

- calocephala $\boldsymbol{H}$

- cicutaefolia Horn.

- Crecodilium $\boldsymbol{L}$.

- Crupina $L$.

- depressa M. B.

- eriophera $\boldsymbol{L}$. glastifolia $\boldsymbol{L}$. macrocephala. melitensis $\boldsymbol{L}$. moschata $L$ ragusina $L$. 
Ribes anrenm Pu/a Lam. Richardia scabra Lam. Rivina laevis $I$ olia $L$. Rosa rubifolia rea $W$ Rudbeckia anca $L$.

- lacifins angustifolius Dec. -introspermum Chrysanthemum Cerastium davuricum Fisch.

dichotomum $L$

- grandiflorum Kit.

- tomentosum $L$.

Ceratochloa pendula Schrad. - unioloides Dec. Cerinthe aspera Roth. - major $L$

Chaerophyllum anreum $L$. Chaiturus Marrubiastrum Rchb. Cheirinia crepidifolia $L k$.

Chelidonium laciniatum Milt. Chelone campanulata Cav.

- diffusa Dougl.

- Digitalis Spr.

- gentianoides.

- glabra $L$

- hirsuta $L$.

- ovata Dougl.

- procera.

Chenopodium Quinoa $\boldsymbol{W}$.

Chorizema ilicifolium Labill.

Chrysanthemum carinatum Schousb.

- disciforme C. A. Mey.

Chryseis tenuiflora $\boldsymbol{H}$. Jen.

Cicer arietinum $\boldsymbol{L}$.

Cicuta maculata $\boldsymbol{L}$

Cineraria speciosa Schrad.

Cinna expansa Link.

Cirsium canum $M$. B.

- laniflorum $M . B$.

- scleranthon $M . B$.

Cistus candidissimus Dun.

- monspeliensis $L$. vaginatus Ait.

Secarikia elegans Dougl.

Sedum nttaicum Bess.

iytonia perfoluata Don.

iematis campaniflora Brot

- lathyrifolin Bess.

- parviflora Dec.

Cleome graveolens Rafin.

- spinosa $\boldsymbol{L}$.

[H. Gryph.

Clintenia elegans Dougl.

Cneorum tricoccon $L$.

Cnicus syriacus $W$.

- tuberosus.

Cnidinm apisides Spr.

Cochlearia officinalis $\boldsymbol{L}$.

Coenolophium Fischeri Koch.

Coix exaltata Jacq.

Collinsia bicolor Benth.

Collomia coccinea Lehm. randifiora Doust.

Colpodium Steveni Trin.

Commelina angustifolia $M x$.

- clandestina Mart.

- coelestis $\boldsymbol{L}$.

- polygama Roth.

Cenringia perfoliata $L k$.

Convolvulas siculus $\boldsymbol{L}$.

Corchorus hirsutus $L$.

- olitorins $\boldsymbol{L}$.

- trilocularis $\boldsymbol{L}$

Coreopsis lanceolata $L$.

- praecex Fresen.

Coriaria myrtifolia $\boldsymbol{L}$.

Corispermum canescens Kit.

Cornucopiae cucullatum $\boldsymbol{L}$.

Coronilla coronata $\boldsymbol{L}$.
Coronilla glanea $\boldsymbol{L}$.

- Securidaca $\boldsymbol{L}$

Corydalis aurea $W$

- nobilis Pers.

- sempervirens Pers.

Cotyledon Umbilicus $L$

Cousinia Hystrix C. A.Mey.

Crambe grandiflora Stev.

- maritima $L$.

Crepis heterosperma Schrad.

- lacera Tenor.

Crotalaria incana $L$.

- purpurea Vent. sagittalis $\boldsymbol{L}$.

Crucianella angustifolia $L$.

- patula.

Cryptostemma calendulacea $R . B r$.

Cryptotaenia Thomasii Dee.

Cucubalus bacciferus $L$ catholicus $L$.

Cucnrbita lagenaria $\boldsymbol{L}$.

Cuphea viscosissima Jacq.

Cyclachaena xanthifolia Fresen.

Cylista villosa Ait.

Cynanchum fuscatum Link.

- nigrum Pers.

Cynoglossum apenninum $\boldsymbol{L}$.

- bicolor $W$.

- canescens $\boldsymbol{W}$.

- sylvaticum $\mathbf{S} m$.

Cytisus capitatus Jacq.

- hirsutus $L$

- prostratus.

Dactylaena micrantha Schrad.

Dalea alopecuroides Nutt.

Daphne Laureola $\boldsymbol{L}$.

- Mezereum $L$.

Datisca cannabina $\boldsymbol{L}$.

Datura ceratocaula Jacq.

- Tatula $L$

Daucus hispidus Desf.

- maritimus $\boldsymbol{L}$.

- muricatus $L$.

- parviflorus Desf.

Decaneurum senegalense Dec.

Delphinium grandiflorum $\boldsymbol{L}$.

- montanum Dec.

- Requienii Dec.

Staphisagria $L$.

Deschampsia juncea Beauv.

Desmanthus virgatns $\boldsymbol{W}$.

Dianthus dintinus Kit.

Dictamnus albus $L$.

Didiscus caeruleus Hook.

Digitalis Buxbaumii Bess.

- ferruginea $L$.

- grandiflora Lam.

- lanata Ehrh.

- Iutea $\boldsymbol{L}$.

- Sceptrum $L$.

Digitaria aegyptiaca $W$.

Dinebra curtipendula Dec.

Diplachne fascicularis $P$. $B$.

Diplophyllum veronicaeforme

Diplostephium amygdalinum Cass. Dipsaens ferox Lois.

- Gmelini M. $B$.

pilosus $\boldsymbol{L}$.

Dolichos purpureus $L$.

Doryenium herbaceum Vill.

Draba aurea $\mathrm{Vahl}$. contorta Ehrh. fladnizensis $W$ ulf. Iutea Gilib.

Dracocephalum altaiense Laxm.

- canariense $\boldsymbol{L}$. canescens $\boldsymbol{L}$. grandiflorum $L$. sibiricum $\boldsymbol{L}$.
Dracocephalum thymiflorum $L$. Drummondia mitelloides Dec.

Echinacea purpurea Moench.

Echinocactus Sellovii.

Echinops Ritro $L$.

Echinospermum Lappula Lehm.

virginicum Lehm.

Eclipta erecta $L$. - latifolia $L$.

Elephantopus carolinianus $\boldsymbol{W}$.

Eleusine coracana Pers.

- indica Lam.

- rigida Spr.

- Tocussa Fres.

Elichrysum bracteatum Vent.

- fulgidum $W$.

Elsholtzia cristata $W$.

Elymus arenarius $\boldsymbol{L}$

- canadensis $L$.

- carolinianus.

- giganteus Vahl.

Emex spinosus Camp.

Entelea arborescens $\boldsymbol{R}, \boldsymbol{B r}$.

Epilobium angustissimum Ait.

Eragrostis abyssinica $L k$. namaquensis Nees ab Es.

Eranthis hyemalis Salisb.

Eremogone graminea C. A. Mey.

Erigeron contortus Desf.

- elongatus Led.

- glabellus Nutt.

- podolicus Bess.

- purpureus Ait.

- Villarsii Bell.

Eriophyllum caespitosum Dougl.

Erodium gruinum Ait.

- melanostigma Mart.

- moschatum Ait.

- pulverulentum $W$.

Ervum agrigentinum Guss.

- Ervilia L.

Eryngium giganteum $M . B$.

Erysimum altaicum Meyer.

- dubium Dec.

- hieracifolium.

- odoratum Ehrh.

Eschscholzia californica Cham

Eucharidium concinnum Fisch. et v.

Eupatorium album $\boldsymbol{L}$.

Euphorbia cyathophora Jacq.

- Humboldtii $W$.

- Lagascae Spr.

- Lathyris L.

- prunifolia Jacq.

Eustachys petraea Desv.

Eutoca viscida Benth.

- Wrangeliana $F$. et $M$.

Exacum viseosum $S \boldsymbol{m}$.

Farsetia clypeata $\boldsymbol{R} . \mathbf{B r}$.

- eriocarpa Dec.

Fedia Cornueopiae Vahl

Ferula campestris Bess.

- Ferulago $\boldsymbol{L}$.

Flaveria repanda $\mathrm{Lag}$.

Francoa appendiculata $\mathrm{Cav}$. sonchifolia Spr.

Fuchsia arborescens Sess. coccinea $L$

Fumaria eapreolata $L$

Galardia bicolor Lam.

Galega orientalis $\mathbf{L a m}$.

- persica Pers.

- stricta Ait.

Garidella Nigellastrum $\boldsymbol{L}$.

Galinsogea trilobata Cav.

Galium verrucosum $S m$.

Gasteria obliqua Haw.

Gaura biennis $\boldsymbol{L}$.

- mutabilis Cav.

Genista ovata $W$. et $K$.

Gentiana cruciata $L$.

Geranium carolinianum $L$.

- sanguineum $L$.

Geropogon glaber $L$.

Geum album Gmel.

- atlanticum Desf.

- coccineum Sm.

- heterophyllum.

- japonicum Thunb.

- macrophyllum $W$.

- ranunculoides Ser.

Gilia achilleaefolia Benth.

- capitata Hook.

- geminiflora Hook.

- inconspicua Hook.

- laciniata $R$. et $P$

- tricolor Benth.

Gladiolus psittacinus Hook.

Glaucium luteum Scop. violaceum $\boldsymbol{S} m$.

Glycine Comptoniana Andr.

Glycyrrhiza echinata $L$ foetida Desf.

glabra $\boldsymbol{L}$.

glandulifera $\mathrm{Kit}$.

- lepidota Pursh.

Gnaphalium foetidum $L$

Goodenia grandiflora Sims.

Grahamia aromatica Hook.

Grindelia incisa $\boldsymbol{S p r}$.

Gypsophila acutifolia Fisch

- altissima $L$.

- collina Stev.

- elegans $M . B$.

- glauca Stev.

- repens $L$.

- Rokejeka Del.

- Steveni Fisch.

- stricta Bunge.

Hablizia tamnoides $M . B$.

Haloragis Cercodia Ait.

Hasselquistia cordata $L$.

Hedypnois pendula $W$.

Hedysarum alpinum $\boldsymbol{L}$. canadense $\boldsymbol{L}$ elongatum Fisch.

flexuosum $L$. sibiricum Lam.

Heimia myrtifolia $L k$

- salicifolia $L k$

Helenium autumnale $L$

- californicum Dougl.

- mexicanum Humb. 
Hieracium rotundifolium Kit. villosum $\boldsymbol{L}$

Hiorthia anrea Less.

Hippocrepis biflora Jacq.

ciliata $W$

- multisiliqua $\boldsymbol{L}$.

- unisiliquosa $L$

Humea elegans $S m$.

Hyoscyamus aurens $L$.

- canariensis Ker.

- physaloides $\boldsymbol{L}$

- pusillus $L$.

Hyoseris arenaria Schousb.

scabra $L$ tubaeformis Ten.

Hypecoum procumbens $L$

Hypochoeris arachnites Biv.

Hyssopus Foeniculum Spr.

- Lophanthus $L$.

- nepetoides $L \$$

- officinalis $\boldsymbol{L}$.

- orientalis $\boldsymbol{W}$.

- scrofularifolius $W$.

Iberis pinnata $L$. umbellata $\boldsymbol{L}$.

Impatiens parviflora Dec.

Indigofera Anil $\boldsymbol{L}$. australis $W$

Inula bifrons $\boldsymbol{L}$. odora $L$. suaveolens Jacq.

Ipomoea coccinea $L$. hederacea Jacq

Iris Güldenstãdtiana Lepech.

- maritima Mill.

- manritiana.

- ochroleuca $M . B$.

- spuria.

- $\quad$ - $\beta$. minor

- stenogyna Dec.

- virginica $L$.

Isatis bannatiea

- dasycarpa Led.

- hebecarpa Dec.

- heterocarpa Andrz. latisidiqur Stev.

Ismelia versicolor Cass.

Isopyrum fumarioides $L$.

Isotoma axillaris Lindl.

Juncus tenuis $W$.

Justicia sarmentosa.

Ixia Bulboendium.

Kalbfussia Milleri Schultz.

Kanlfussia amelloides Nees.

Kennedia apetala Lodd.

- monophylla Vent.

- prostrata $\boldsymbol{R}$. $B r$. Pent.

Kitaibelia vitifolia $W$

Knantia orientalis $\boldsymbol{L}$,

Kochia eriophora Schrad.

- hyssopifolia Roth.

- scoparía Schrat.

Koeleria glauca Dec.

- laxa Link.

Kuhnia suaveolens.

Lactuca crispa Roth. perennis $L$.

Lagoecia cuminoides $L$. Lamarekia atren Möneh.

Lamium garganicum $\boldsymbol{L}$.

Lantana salviaefolia Jacq.

Lappage racemosa Schreb.

Lapsana grandiflora $\boldsymbol{M}$. B.

Laserpitium angustifolium. - pilosum $W$.

Lasiopetalum purpureum Sims Lasiospermum radiatum Trev. Lathyrus angulatus $\boldsymbol{L}$

- ennnus $L$.
Lathyrus Aphaca $L$.

- Clymenum $L$.

- latifolins $\boldsymbol{L}$.

- longipedunculatus Led.

- magellanicus Lam.

mexicanus Wender.

- Nissolia $L$.

- Ochrus Dec.

- speciosus $L$.

Lavandula latifolia Ehrh.

- Stoechas $\boldsymbol{L}$.

Lavatera arborea $L$. plebeja Sims.

Leonurus nepalensis.

Lepidium affine $L e d$. suffruticosum $L$.

Lepidonema chilensis Fisch. et $M$.

Lestiboudesia virgata Jacq.

Leucocarpus alatus Don.

Levisticum officinale Koch.

Liatris spicata $W$.

Libanotis Buchtormensis Dec.

- vulgaris Dec.

Ligusticum alatum $S p r$. Seguieri Koch.

Linaria bipartita $W$. - genistaefolia Mill.

- litoralis Bernh.

- purpurea Mill.

- pyrenaica Dec. triphylla $\boldsymbol{W}$

Lindenbergia urticaefolia Lehm.

Linum aquilinum Molin.

- flavum $\boldsymbol{L}$.

- grandiflorum Desf. sibiricum Dec.

Lobelia alata $R$. Br.

- Erinus $L$.

- inflata $L$

- syphilitica $\boldsymbol{L}$.

triquetra $L$.

Lolium italicum A. Braun. peciosum Stev.

Lomatophyllum borbonicum $W$.

Lopezia coronata $A n d r$.

Lophospermum scandens Don.

Lotus argenteus.

- edulis $\boldsymbol{L}$

- cytisoides $\boldsymbol{L}$.

- glanens Ait.

- Jacobaeus $L$.

- ornithopodioides $L$.

- siliquosus $L$.

- suaveolens Pers.

Lupinus albus $\boldsymbol{L}$.

- angustifolius $\boldsymbol{L}$

- bicolor Lindl.

- canaliculatus Sweet.

- Cruikshankii Bot. Mag.

- Lehmanni Mïll.

- lituratus Reichb

- micranthus Dougl.

- nanus Benth.

- pilosus $L$

- polyphyllus Dougl. pulchellus Dougl. succulentus Lindl.

- Termis Forsk. tomentosus Sweet.

Luzula maxima Dec.

Lychnis brachypetala Fisch.

- Coeli rosa Desr.

- fulgens Fisch.

- inclusa Lehm. nemoralis Heuff.

Lycopus exaltatus $L$. virginieus $L$.

Lysimachia ciliata $L$.

- dubia Ait.

- Ephemerum $L$
Lysimachia verticillata Pall.

Lythrum verticillatum $L$. - virgatum $L$.

Macrorhynchus pterocarpus $\boldsymbol{F}$, et $\mathbf{M}$. Madia elegans Don.

- quinqueradiata Fisch. et $M$.

Malcolmia arenaria Dec.

- maritima $\boldsymbol{R}$. $B r$.

Malope grandiflora Hort. malacoides $L$.

Mandragora officinalis Mill.

Marrubium africanum $L$.

- astracanicam Jacq.

- hirsutum $W$.

- propinquum $F$. et $M$.

Maurandia antirrhiniflora Humb.

Meconopsis cambrica $\mathrm{Vig}$.

Megastarhya rigida Beauv.

Melica altissima $\boldsymbol{L}$.

- ciliata $L$.

Melilotus Bonplandii Ten. caerulea Desv.

, var. connata.

- indica Desv.

- rotundifolia Ten.

- rugulusa $u$.

- ruthenica $M, B$.

Melissa alba Kit.

Menispermum canadense $\boldsymbol{L}$.

Mesembrianthemum pugionifor-

Meum athamanticum Jacq me $L$.

Mimulus cardinalis Lindl.

- floribundus Lindl.

- Intens $L$.

- moschatus Dougl.

- roseus.

Mirabilis dichotoma $\boldsymbol{L}$.

- Jalappa $\boldsymbol{L}$.

- longiflora $L$.

Mogiphanes virgata Schrad.

Molinia altissima Link.

Mollia diffusa $W$.

- latifolia $\boldsymbol{U}$

Molopospermum peloponnesiacum

Moluccella laeris $\boldsymbol{L}$.

Momordica Elaterium $\boldsymbol{L}$.

Moraea chinensis Thunb.

Mühlenbergia diffusa Schreb.

Muscari comosum $W$.

Myagrum perfoliatum $L$.

Myrrhis aromatica $S_{p}$ reng.

- odorata Scop.

Nemophila Atomaria $F$, et $M$.

- insignis Dougl.

- peduneulata Dougl.

- phacelioides Bart.

Nepeta coerulea Ait.

- colorata $W$

- fissa C. A. Mey.

- grandiflora.

- italica $L$

- longiflora Vent.

- marrnbioides $U$.

- pannonica Jacq.

- teucrioides Lam.

- neranica $L$

Nicandra physalodes Gaertn.

Nicotiana alata $L k$. et Otto.

- chinensis Fisch.

- fruticosa $L$.

- glauca Hook.

- glntinosa $L$

- Langsderffii Nees.

- macrophylla Spr.

- noctiflora Hook.

vineaeflora $\mathrm{Lag}$.

Nigella orientalis $\boldsymbol{L}$.

- sativa $L$

Nuttallia malvaed

Obeliscaria pinnat

Odontarrhena obovat

Oenothera eruciata

Fraseri Pursh,

glauca $M x$

Lindleyana Sims.

muricata $L$.

odorata Jacq.

pumila $L$.

purpurea Curt.

rosea Ait.

spectabilis Horn.

triloba Nutt.

undulata Ait.

Omphalodes linifolia Moench.

Onobroma creticum $5 \mathrm{pr}$.

- lanatum $s p r$.

Onobrychis Caput galli Lam.

- conferta Spr.

Crista galli Lam.

Ononis alopecuroides $L$.

hireina.

Natrix $L$

Onopordon illyricum $L$. macracanthum Schousb. viseosum.

Ornithogalum Gussoni Ten. narbonense $\boldsymbol{L}$. pusillum.

Orobus niger $L$.

- vernus $L$.

Oxyria reniformis Hook.

Paeonia officinalis $\boldsymbol{L}$.

- peregrina Mill.

- tennifolia $L$

- triternata Pall.

- villosa.

Panicum plicatum Lam.

Papaver bracteatum Lindl.

- hybridum $L$.

- intermedium $A l p h . D e c$.

- orientale $L$.

somniferum

tauricand

Pascalia glauea Orteg.

Paspalum elegans Elügg.

- scrobiculatum $\boldsymbol{L}$.

- stoloniferum Bosc.

Passiflora edulis Sims.

- gracilis Jacq.

- heterophylla. 
Ribes anreum $\boldsymbol{P}_{\boldsymbol{u}}$ anum Desf.

Richardia seabre a Del.

Rivina laevis $I^{a}$ M. B.

Rosa rubifolia pacea Labill.

Rudbeckia an Anisum $\boldsymbol{L}$. - lacis regrina L. efmin sativum $L, \gamma$ umbellatum.

Pittosporum undulatum Andr. Plumbargo micrantha Desf. Pocochia cretica Ser. Pedalyria anstralis $W$.

Podospernmin laciniatum Dec.

Polenonium coernlenm $\boldsymbol{H}$. - disaectum Reiehb.

Polygonum eymosum Trev emarginatim Rath nudulation - Murr

- viviparum $l$.

Pomaderris Detulina Cun. - elliptica Lab. - phylieaefolia $L k$.

Portulaea graudiflora Hool:

Potentilla arguta Pursh.

- atrosanguinea Don.

- calabra Ten.

- formesa Don.

- gracilis Dougl.

- ontepeda Lehm.

- recta $L$

- rupestris $L$.

- Thomasii Ten.

- viseosa Don.

Poterium garganieum Ten.

- guestphalieum Bönn.

- spinosum $L$.

Primula chinensis.

- inflata Lehm.

- verticillata Forsk.

Prismatocarpos faleatus Ten.

Prunella alba Pall.

- grandiflora $\boldsymbol{L}$. hyssopifolia $L$ pensylvanica $W$

- -alea bituminosa $L$.

Secarrikmuracteata In

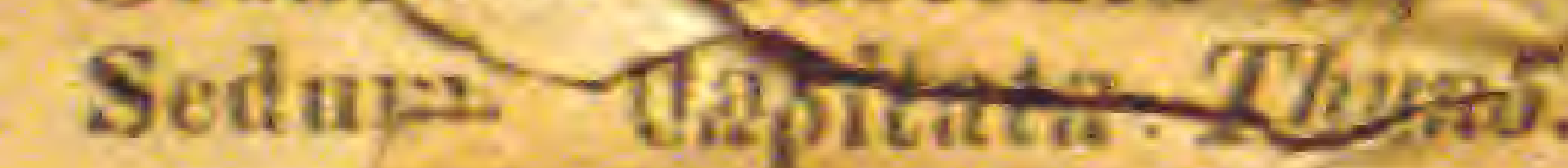

Eckloni Ott.

hiria L.

pinnata $L$

polystrachya Poir.

errucosa $W$.

Ptychotis heterophylla Koch.

Pycnanthemum incaram Mich.

Pyrethrum anethifolimn $W$.

- corymbosum $\|$.

- indiemm Roxb.

- mactophyllum $I F$

- roillefoliatum $W$.

- roseum M. B.

- tenuifolium Ten.

Ranuneuins cancasicus $\boldsymbol{M}$. $B$.

- crassicaulis.

- illyricus $\boldsymbol{L}$.

- millefoliatus Tahl.

- muricatus $L$.

- nemorosas Dec.

- pedatus Kit.

- Sterenii Besg.

- tuberculatus Dec.

Reseda alba $\mathrm{L}$.

- crispata $L k$.

- Pliyteuma b.

Bhagadiolus edulis Gaerin.

- Koelpinia $\boldsymbol{W}$

- stellatris of.
Ribes aureum Pursh.

Richardia scatbra $L$.

Rudbeekia amplexifolia Jacq.

- asperrima Horn.

- laciniata $L$

- speciosa Wender. triloba $\boldsymbol{L}$.

Rumex hamatus Trev.

- salieifolius $W$ einm.

Ruta chalepensis $\boldsymbol{L}$.

Salsola baceata Poir. Tragus $L$.

Satvia indica $\boldsymbol{L}$.

- interrupta Schousb.

lanceolata Cav. tingitana Etling. virgata Ait.

Sambucus canadensis $\mathbf{L}$.

Samolus Valerandi $L$.

Sauieuta marilandica $L$.

Sanvitalia procambens $W$.

Saponaria calabrica Guss.

- cerastoides Led. Vacearia $\boldsymbol{L}$.

Saracha viscosa Schrad.

Satureja cuneifolia $T e n$.

- montana $L$

Saxifraga dentata $L k$.

- Ponae Sternb.

- pyramidalis Lap. rotundifolia $\boldsymbol{L}$

Scabiosa graminifolia $\boldsymbol{L}$.

Scandix brachyearpa Guss.

Schismns marginatus $\boldsymbol{P} . \boldsymbol{B}$.

Schiverenkia podolica Andro.

Schizantlus pinnatus $\boldsymbol{R}$. et $\boldsymbol{P}$.

Scolymus maenlatus $\boldsymbol{L}$.

Scoparia dulcis $\boldsymbol{L}$.

Seorpiurus muricata $L$.

- sulcata $L$.

- vermiculata $L$.

Serofularia Balbisii Horn.

- cordata Pers.

- mellifera Ait.

- peregrina $L$.

- Seorodonia $L$.

- variegata $M . B$.

- vernalis $\boldsymbol{L}$.

Scutellaria Iateriflora $\boldsymbol{L}$.

- peregrina $L$.

- rubieunda Horn.

Secale fragile $M$. $B$.

Sedum altaicum Bess.

- hybridum $\boldsymbol{L}$.

- Rhodiola Dec.

Selago bracteata Thunb.

- fasciculata $\boldsymbol{L}$.

Senecie Doria $L$.

- elegans $L$.

- lilacinas Selerad.

- ovatus IV.

- purpureus $L$.

Seriola aethnensis $L$.

Serratula alata $H$.

- cynaroides Dec. radiata $M . B$

Seseli glanenm $L$.

- gummifernm $S_{m}$.

- rigidum fit.

- tortuosnm $L$.

Sesleria coerulea Ard.

- elongata Host.

mitida Ten.

Setaria italica Kunth.

- pirpurnscens $\boldsymbol{H}$. et $\boldsymbol{K}$.
Sida Abutilon $\boldsymbol{L}$.

- angustifolia Juss.

- indiea $L$.

- Napaea Cav.

- triloba Cav.

- vesicaria Cav.

Sideritis montana $L$.

Siegesbeckia flosculosa Ilerit.

- orientalis $\boldsymbol{L}$.

Silene alpestris Jacq.

- Atocion Jacq.

- chlorantha Ehrh.

- compacta Horn.

- cretica $L$.

- flavescens $\boldsymbol{K} i t$.

- fruticosa $L$.

- gallica $L$

- infraeta $K i t$.

- linaccola $W i b$.

- livida IV.

- mollissima $S m$.

- pendula L.

- quinquevulnera $L$.

regia Sims.

saponariafolia Sehott.

serieca All.

viridiflora $L$.

Sisymbrium austriacnm Jacq. strictissimum $L$.

Sisyrinchium Bermudiana $\boldsymbol{L}$. striatum Cav.

Solanum campechiense $L$.

- coccinenm Jacq.

- grandiflorum $\boldsymbol{R}$. et $\boldsymbol{P}$.

- pyracanthum Lam.

- Thoningiannm Jacq.

Sonchus floridanus $\boldsymbol{L}$. leneophaeus $W$.

Spartina cynosuroides Roth.

Spartium junceum $\boldsymbol{L}$.

Specularia biflora $\boldsymbol{F}$. et $\boldsymbol{M}$.

Spermacoce longifolia $A u b l$.

Spilanthes oleracea $L$.

Stachys alpina $L$.

- cretiea $L$.

- grandiflora Host,

- lanata Jacq.

- polystachya Ten.

- setifera C. A. Mey.

Stachytarpheta jamaicensis lahl.

Statice sinnata $\boldsymbol{L}$.

Stenactis speciosa Lindl.

Stipa capillata $\boldsymbol{L}$.

- gigantea $L a g$.

Stylidinm adnatum $\boldsymbol{R}$. $\boldsymbol{B r}$.

Sutherlandia frutescen $\boldsymbol{R}, \boldsymbol{B r}$.

Swainsonia coronillaefolia Salisb.

Symphytum asperrimum $M$. $B$.

Syrenia Biebersteinii Andrz.

Tagetes lucida Cav.

Tantcetum boreale.

Teedia Iucida Rud.

Telekia eordifulia $\boldsymbol{k}$ it.

Telephinm Imperati $\boldsymbol{L}$.

Tellima grandiffura Lindl.

Tetrapoma barbareaefolium Turez

Tenerinm Arduini $L$.

- flavum $L$.

- hyreanicum $\boldsymbol{L}$.

- massiliense $L$

- Prenda-Scorodonia Desf.

Thalictrum aquilegifolium $\boldsymbol{L}$.

Thrincin hirta $R_{0} h$

Thrmbergia alata Hook.

Tiaridinm indien Lehm.

Tolpis barbata Gaerín.

Tordylium maximum $\boldsymbol{L}$. syriacum $L$.

Tradescantia erecta Cav. Selloviana $P o h l$

Tragopogon eriospermus $T$ en.

- floccosus Kit.

- porrifolius $L$.

Tribulus terrestris $\boldsymbol{L}$.

Trichodium laxiflorum Mich.

Tricholaena rosea Nees.

Trichonema ramiflora $T e n$.

Trifolium bracteatum Schousb.

- incarnatum $\boldsymbol{L}$.

- libanoticum Ehrenb.

- ligusticum Balb.

- subterraneam $L$.

- vesiculosum Sant.

Trigonella arcuata Meyer, Callicezas Fisch.

- cancellata Fisch.

- Foenum graecum $\boldsymbol{L}$.

- tenuis Fisch. uralensis Led.

Urachne paradoxa $\boldsymbol{L} k$,

Urospermum picroides Desf.

Urtica cannabina $\boldsymbol{L}$.

- convexa Horn. pilulifera $L$.

Valexianella coronata Dec.

- gracilis Fisch, et Mey.

- hamata Dec. uncinata Dufr.

Verbena Aubletia Jacq.

- bonariensis $L$.

- oracteosa $M x$.

- hastata $L$.

- lasiostachys $\boldsymbol{L} k$.

- paniculata Lam.

- stricta Fent.

- urticaefolia $\boldsymbol{L}$.

- venosa Gill. et Hook.

Veronica brevifolia $\boldsymbol{M}$. $\boldsymbol{B}$.

- caucasica $M$. B.

- gentianoides $V a h l$.

- prostrita $L$

- sibirica $\boldsymbol{L}$.

- virginica $\boldsymbol{L}$.

Vesicaria sinuata Poir.

Vicia articulata $L$.

- biennis $L$.

- bithynica $\boldsymbol{L}$.

- disperma Dec.

- globosa Reta.

- lutea $L$.

Viminaria denudata $5 \mathrm{~m}$.

Viola hederacea Lrbill.

Wahlenbergia grandiflora Scluad.

Xanthium orientale $\boldsymbol{l}$. spinosum $L$.

Xeranthemum annumm $\boldsymbol{L}$.

- erectum Presl.

- inapertum Dec.

Ximenesia encelioides $\mathrm{Cat}$.

Zaeyntha verrucosa Gârtn.

Zephyranthes candida Herb.

Zinnia elegans var, violacea. 